\title{
Low Phase Noise Cylindrical Cavity Oscillator
}

\author{
by
}

Jacques Maree

Thesis presented in partial fulfilment of the requirements for the degree Master of Science in Engineering at Stellenbosch University

Supervisor: Prof J.B. de Swardt Co-supervisor: Prof P.W. van der Walt

Department of Electrical \& Electronic Engineering

March 2013 


\section{Declaration}

By submitting this thesis electronically, I declare that the entirety of the work contained therein is my own, original work, that I am the sole author thereof (save to the extent explicitly otherwise stated), that reproduction and publication thereof by Stellenbosch University will not infringe any third party rights and that I have not previously in its entirety or in part submitted it for obtaining any qualification.

Jacques Maree

March 2013

Copyright (C) 2013 Stellenbosch University

All rights reserved 


\section{Abstract}

The objective of this thesis is to develop a $9.2 \mathrm{GHz}$ low phase noise oscillator with a cylindrical cavity resonator.

A cylindrical metal cavity with air as dielectric was used as a resonator. To minimise the phase noise of the oscillator, the resonator must be designed to have a high Q-factor. A high Q-factor was obtained by designing the resonator to operate in the $\mathrm{TE}_{011}$ mode. A tuning screw was used to tune the resonant frequency without significantly affecting the $Q$-factor. The tuning screw also separates the resonant frequencies of the degenerate $\mathrm{TE}_{011}$ and $\mathrm{TM}_{111}$ modes. The signal is coupled to the resonator by means of rectangular apertures. The coupling was designed to minimise the phase noise of the oscillator.

A dual mode waveguide filter was developed and inserted into the oscillator loop in order to prevent oscillation at unwanted frequencies.

Due to the excellent phase noise performance of the oscillator, it was not possible to measure the phase noise directly with the available phase noise meter. A measurement setup using two similar oscillators tuned to oscillate at frequencies differing by about $60 \mathrm{MHz}$ was implemented. The output signals were down-converted to the difference frequency where the phase noise could be measured accurately.

The output signal of the oscillator was measured at different locations in the loop and clearly showed that the resonator can be used as a filter to minimise the phase noise.

The performance of the oscillators met all expectations. Phase noise levels of $-115 \mathrm{dBc} / \mathrm{Hz}$ and $-146 \mathrm{dBc} / \mathrm{Hz}$ were obtained at offset frequencies of 10 and $100 \mathrm{kHz}$. 


\section{Opsomming}

Die doel van hierdie tesis is om ' $\mathrm{n} 9.2 \mathrm{GHz}$ lae faseruis ossillator met ' $\mathrm{n}$ silindriese holte resoneerder te ontwikkel.

' $n$ Silindriese metaal golfleier holte met ' $n$ lug diëlektrikum was gebruik as die resoneerder. Om die faseruis van die ossillator te minimeer moet die resoneerder ontwerp word om ' $n$ hoë $Q$-faktor te hê. Om ' $n$ hoë $Q$-faktor te behaal was die resoneerder ontwerp om in die $\mathrm{TE}_{011}$ orde te werk. Die resoneerder is toegerus met ' $n$ verstelskroef wat die bedryfsfrekwensie verstel sonder om die belaste $Q$-faktor aansienlik te beïnvloed. Die verstelskroef skei ook die frekwensie van die degeneratiewe $\mathrm{TE}_{011}$ en $\mathrm{TM}_{111}$ ordes. Drywing word na die resoneerder gekoppel deur middel van reghoekige openinge. Die koppeling is ontwerp om die faseruis van die ossillator te minimeer.

' $n$ Tweede orde dubbelmodes golfleier filter is ontwerp en in die ossillatorlus ingevoeg om ossillasie by ongewenste frekwensies te voorkom.

Vanweë die baie lae faseruis van die ossillator was dit nie moontlik om die faseruis direk met die beskikbare faseruismeter te meet nie. ' $n$ Meetopstelling met twee soorgelyke ossillators waarvan die frekwensies met ongeveer $60 \mathrm{MHz}$ verskil is geïmplementeer. Die uittreeseine van die ossillators is afgemeng na die verskilfrekwensie waar die meetinstrument meer sensitief is en die faseruis akkuraat gemeet kan word.

Die uittreesein van die ossillator is by verskillende punte gemeet en het duidelik getoon dat die resoneerder as filter gebruik kan word om die faseruis te minimeer.

Die ossillators se werkverrigting het aan die verwagtinge voldoen. Faseruis vlakke van $-115 \mathrm{dBc} / \mathrm{Hz}$ en $-146 \mathrm{dBc} / \mathrm{Hz}$ by afsetfrekwensies van onderskeidelik $10 \mathrm{en} 100 \mathrm{kHz}$ is verkry. 


\section{Acknowledgements}

I would like to take this opportunity to thank everyone who was involved in this making this project possible and successful.

A special thanks to my mother and father whom supported me emotionally and also financially throughout my years of study and throughout the whole of this project. Without you it wouldn't have been possible for me to have come this far.

Thank you Prof J.B. de Swardt, my supervisor, for all you help, guidance and encouragement throughout the whole of the project and for always making time for me whenever I had a question or a concern. You truly are a remarkable supervisor.

Thank you to my co-supervisor Prof P.W. van der Walt for all your input and technical insight. It has been an honour working alongside such a brilliant mind.

Thank you to Wessel Croukamp and Lincoln Saunders, the technical staff at the electronic workshop of the University of Stellenbosch, who manufactured the designed components with precision and timely. A special thanks to Wessel Croukamp for your insight in manufacturing components whenever difficulties arised and being friendly and helpful whenever help was needed.

Thank you Rob Anderson, manager of the RF laboratory of the University of Stellenbosch, for your help with the measurements whenever needed and for all the advice and technical insight that you provided.

Much gratitude to Reutech Radar Systems (RRS) for providing me with the financial support to carry out this project.

Also thank you to Susan Maas, Martinette van der Merwe and Westley Francis, employees at RRS, for all your help whenever I needed components, make measurements and for being friendly and helpful whenever I needed help. I truly appreciate it. 


\section{Table of Contents}

Declaration

Abstract

Opsomming iii

Acknowledgements iv

Table of Contents $\quad$ v

List of Figures viii

List of Tables $\quad$ xiii

$\begin{array}{ll}\text { Nomenclature } & \text { xiv }\end{array}$

List of Appendices $\quad$ XV

1 INTRODUCTION

1.1 Problem statement 1

1.2 Proposed solution 1

1.3 Overview of thesis 2

2 OSCILLATOR THEORY 3

2.1 Fundamentals 3

2.2 Microwave and RF oscillators 5

2.2.1 Method 1: Open-loop gain and phase 5

2.2.2 Method 2: One-port negative-resistance device method 6

2.3 General oscillator elements 9

2.4 Types of high Q-factor microwave resonators and their applications 10

2.5 Resonator losses and the Q-factor 11

2.6 Noise in oscillators 13

$\begin{array}{lll}2.6 .1 & \text { Amplitude noise } & 14\end{array}$

2.6.2 Phase noise 15

$\begin{array}{lll}\text { 2.6.2.1 Characterization of phase noise } & 17\end{array}$

$\begin{array}{lll}\text { 2.6.2.2 } & \text { Power-law phase noise distribution } & 18\end{array}$

2.6.2.3 The Leeson formula for phase noise distribution 20

2.7 Configurations and techniques to reduce phase noise in microwave oscillators 22 
$\begin{array}{llr}3.1 & \text { Resonator } & 27\end{array}$

3.1.1 Theory 27

$\begin{array}{lll}3.1 .2 & \text { Design } & 31\end{array}$

3.1.2.1 Separating degenerate modes and tuning centre frequency of $\mathrm{TE}_{011}$ mode 33

3.1.2.2 Coupling and excitation 35

3.1.3 Simulations 42

3.1.3.1 Coupling 42

3.1.3.2 Tuning Range 46

$\begin{array}{lll}3.2 & \text { Filter } & 48\end{array}$

$\begin{array}{lll}3.2 .1 & \text { Design } & 48\end{array}$

$\begin{array}{lll}\text { 3.2.1.1 Cavity dimensions } & 48\end{array}$

$\begin{array}{lll}\text { 3.2.1.2 Coupling } & 49\end{array}$

$\begin{array}{lll}\text { 3.2.1.3 Simulations } & 50\end{array}$

4 MEASUREMENTS $\quad 54$

4.1 Resonator $\quad 54$

4.1.1 Tuning range of unplated resonator 56

4.1.2 Tuning range of silver plated resonator 58

$\begin{array}{lll}4.2 & \text { Filter } & 60\end{array}$

$\begin{array}{lll}\text { 4.2.1 Tuning range } & 61\end{array}$

$\begin{array}{lll}4.3 & \text { Oscillator } & 63\end{array}$

4.3.1 Single oscillator measurements 63

4.3.1.1 VNA measurement 64

4.3.1.2 Spectrum analyser measurement $\quad 65$

$\begin{array}{lll}\text { 4.3.1.3 Phase noise measurement } & 67\end{array}$

4.3.2 Two-oscillator measurements $\quad 67$

$\begin{array}{lll}\text { 4.3.2.1 Configuration } 1 & 69\end{array}$

$\begin{array}{lll}\text { 4.3.2.2 Configuration } 2 & 73\end{array}$

4.3.2.3 Measurements using a different active device 76

4.3.3 Comparison to commercially available oscillators and an ultra-low-noise STALO 77

5 CONCLUSIONS AND RECOMMENDATIONS

$\begin{array}{llr}5.1 & \text { Conclusions } & 80\end{array}$

5.2 Recommendations for future work 80 
6 BIBLIOGRAPHY

7 APPENDICES

A-1 


\section{List of Figures}

Figure 2.1: Block diagram of oscillator using amplifier and frequency dependent element in feedback path.

Figure 2.2: Schematic of oscillator that will be used in the following design methods. 5

Figure 2.3: Open-loop oscillator used to meet the Barkhausen criteria using MWO.

Figure 2.4: Simulation response of Figure 2.3 obtained from MWO. The circuit will oscillate where the phase is exactly zero degrees while the gain is larger than unity

Figure 2.5: Negative resistance oscillator model.

Figure 2.6: Circuit used for method 2. It involves looking into the input ports of the amplifier and resonator, separately, to obtain the frequency where the imaginary parts of the two circuits cancel each other while their resulting real part is negative. This will be the frequency of oscillation.

Figure 2.7: $\mathrm{R}_{\text {tot }}$ and $\mathrm{X}_{\text {tot }}$ vs. frequency obtained from MWO. It indicates that the circuit will oscillate at $12.59 \mathrm{MHz}$.

Figure 2.8: General elements forming an oscillator

Figure 2.9: Model of microwave resonator near resonance in a (a) series configuration and (b) parallel configuration.

Figure 2.10: Passband characteristics of resonator. Upper and lower frequency used to calculate half power bandwidth is determined by obtaining the frequencies where the power is $3 \mathrm{~dB}$ down from the maximum transmission power at the centre frequency.....

Figure 2.11: Typical spectrum of oscillator output.

Figure 2.12: Signal containing only amplitude noise.

Figure 2.13: Signal amplitude modulated by a (a) single sinusoid and (b) a concentration of random sinusoids.

Figure 2.14: Time domain signal containing phase noise, illustrating the variation of its phase by looking at the (a) amplitude and (b) phase.

Figure 2.15: Signal phase modulated by a (a) single sinusoid and (b) a concentration of random sinusoids.

Figure 2.16: Phase noise distribution according to the power-law model.

Figure 2.17: Transfer function relating the noise PSD at the input to the noise PSD at the output of an amplifier.

Figure 2.18: Phase noise distribution according to Leeson when $(a) f_{L}>f_{c}$ and $(b) f_{L}<f_{c}$.

Figure 2.19: STALO consisting of a microwave oscillator, phase locked to a high $Q$ resonator [18]. 
Figure 2.20: STALO configuration improved by suppressing the carrier and amplifying the signal before the mixer [18].

Figure 2.21: Phasor diagram showing a frequency error and the effect of suppressing the carrier before entering the RF amplifier [18].

Figure 2.22: Phasor diagram showing the phase noise contributed by the amplifier when the carrier is present and when the carrier is suppressed [18]....

Figure 2.23: STALO configuration designed by Nelson, Howe and Gupta [22].

Figure 2.24: Measured phase noise of STALO in Figure 1-26 [22]......

Figure 3.1: Field configurations of the first 15 modes for a cylindrical waveguide [24].

Figure 3.2: Surface currents obtained from CST simulation of cylindrical $\mathrm{TE}_{011}$ mode (a) on top/bottom lid and (b) on cylindrical outer surface.

Figure 3.3: (a) Top view at $z=d$ and (b) side view of E-field patterns of the cylindrical $T E_{011}$ mode, obtained from a CST simulation.

Figure 3.4: (a) Top view at $\mathrm{z}=\mathrm{d}$ and $(\mathrm{b})$ side view of $\mathrm{H}$-field patterns of the cylindrical $\mathrm{TE}_{011}$ mode, obtained from a CST simulation.

Figure 3.5: (a) Top view at $z=d$ and (b) side view of E-field patterns of the cylindrical $T_{111}$ mode, obtained from a CST simulation.

Figure 3.6: (a) Top view at $z=d$ and (b) side view of $H$-field patterns of the cylindrical $T_{111}$ mode, obtained from a CST simulation.

Figure 3.7: $\mathrm{Q}$ chart of cylindrical waveguide cavity, showing the $\mathrm{TE}_{011}$ mode and the dominant $\mathrm{TE}_{111}$ mode.

Figure 3.8: Cutoff frequencies of different modes of cylindrical waveguide with dimensions of $\mathrm{a}=21.48 \mathrm{~mm}$

Figure 3.9: Resonant frequencies of different modes of cylindrical waveguide cavity with dimensions of $\mathrm{a}=21.48 \mathrm{~mm}$ and $\mathrm{h}=42.96 \mathrm{~mm}$.

Figure 3.10: (a) Top and (b) side view of the surface currents flowing in the $\mathrm{TE}_{011}$ mode cavity, together with the surface currents flowing on the tuning screw.

Figure 3.11: Resonant frequency of the $\mathrm{TM}_{111}$ mode by varying the length of the tuning screw........34

Figure 3.12: Resonant frequency of the $\mathrm{TE}_{011}$ mode by varying the length of the tuning screw.........35

Figure 3.13: Resonant frequencies of different modes with the tuning screw inserted into the cavity at the nominal length $(4 \mathrm{~mm})$.

Figure 3.14: Lumped element model of microwave resonator near resonator, connected to a source and a load, where the transformers represent the coupling.

Figure 3.15: Equivalent model of Figure 3.14 when the circuit is at resonance and the load and source resistances are transformed to the resonator.

Figure 3.16: Plot of (3.22) illustrating that the phase noise with respect to the source conductance reaches a minimum when the derivative is zero. 
Figure 3.17: Unloaded Q-factor of resonator, obtained from a CST simulation.

Figure 3.18: Field configuration of the rectangular $\mathrm{TE}_{10}$ mode [24].

Figure 3.19: (a) Top and (b) side view of structure used in CST for the simulations.

Figure 3.20: S-parameters obtained from a lossless CST simulation using the frequency domain solver.

Figure 3.21: S-parameters obtained from a CST simulation using the frequency domain solver, with losses included.

Figure 3.22: S-parameters of the low end of the tuning range of the resonator obtained from a CST simulation using the frequency domain solver, with losses included. The length of the tuning screw at the low end is at its minimum (zero).

Figure 3.23: S-parameters of the high end of the tuning range of the resonator obtained from a CST simulation using the frequency domain solver, with losses included. The length of the tuning screw at the high end is at its maximum $(12 \mathrm{~mm})$.

Figure 3.24: Wideband simulation of the resonator at its centre frequency obtained from a CST simulation.

Figure 3.25: Field configurations of the rectangular (a) $\mathrm{TE}_{10}$ and (b) $\mathrm{TE}_{01}$ modes [24]

Figure 3.26: E-field patterns in the transverse direction of the rectangular (a) $\mathrm{TE}_{101}$ and (b) $\mathrm{TE}_{011}$ modes.

Figure 3.27: Structure illustrating the E-field patterns of the $T E_{101}$ and $T E_{011}$ modes. Also shown is the position of the coupling screw.

Figure 3.28: Structure illustrating the positions of the input and output probes. Also shown are the tuning screws for the two modes.

Figure 3.29: Filter structure used in CST for simulations.

Figure 3.30: S-parameters of the filter obtained from a lossless CST simulation using the frequency domain solver.

Figure 3.31: S-parameters of the filter obtained from a CST simulation using the frequency domain solver, with losses included.

Figure 3.32: Wideband simulation of the filter at its centre frequency obtained from a CST simulation.

Figure 3.33: Schematic used in MWO of resonator and filter cascaded with a $3 \mathrm{~dB}$ attenuator between them.

Figure 3.34: Simulation obtained from MWO illustrating the response of the cascade with and without the attenuator.

Figure 4.1: Tuneable cylindrical waveguide cavity resonator operating in the $\mathrm{TE}_{011}$ mode with centre frequency at $9.2 \mathrm{GHz}$.

Figure 4.2: Resonator with waveguide transitions and top endplate disconnected. 55

Figure 4.3: Measured S-parameters of resonator tuned to its lowest operating frequency. 
Figure 4.4: Measured S-parameters of resonator tuned to its centre frequency..............................56

Figure 4.5: Measured S-parameters of resonator tuned to its highest operating frequency. .57

Figure 4.6: Wideband measurement of resonator tuned to its centre frequency.

Figure 4.7: Measured S-parameters of resonator plated with silver, tuned to its lowest operating frequency.

Figure 4.8: Measured S-parameters of resonator plated with silver, tuned to its centre frequency.

Figure 4.9: Measured S-parameters of resonator plated with silver, tuned to its lowest operating frequency.

Figure 4.10: Tuneable rectangular waveguide cavity dual mode filter operating in the $\mathrm{TE}_{101}$ and $\mathrm{TE}_{011}$ modes with centre frequency at $9.2 \mathrm{GHz}$. 60

Figure 4.11: Filter with top endplate removed. .60

Figure 4.12: Measured S-parameters of filter tuned to its lowest operating frequency. 61

Figure 4.13: Measured S-parameters of filter tuned to the centre frequency 61

Figure 4.14: Measured S-parameters of filter tuned to its highest operating frequency. .62

Figure 4.15: Wideband measurement of filter tuned to its centre frequency. .62

Figure 4.16: Waveguide cavity oscillator. .63

Figure 4.17: Schematic of open-loop measurement configuration. .64

Figure 4.18: Open-loop measurement setup. 64

Figure 4.19: VNA measurement of open-loop oscillator tuned to operate at its centre frequency. The phase around the loop is tuned to exhibit a zero degree shift at its maximum slope, while the magnitude is larger than unity.

Figure 4.20: Output of oscillator indicating oscillation at $9.2 \mathrm{GHz}$ with a power level of $6 \mathrm{dBm}$. 66

Figure 4.21: Spectrum of oscillator output when the filter is removed from the loop. .66

Figure 4.22: Measured phase noise of oscillator. 67

Figure 4.23: Frequency down-conversion using a mixer. .68

Figure 4.24: Frequency spectrum of down-conversion process. .68

Figure 4.25: Configuration 1: Oscillator configuration with the output taken directly after the amplifier.

Figure 4.26: Close-in spectrum of LO oscillator output. .70

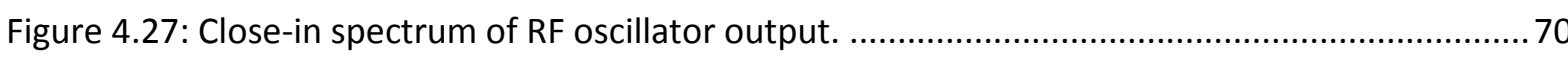

Figure 4.28: Phase noise measurement of LO oscillator. ……......................................................... 71

Figure 4.29: Phase noise measurement of RF oscillator................................................................. 71

Figure 4.30: Phase noise measurement of down-converted RF signal. .72 
Figure 4.31: Configuration 2: Oscillator configuration with the output taken directly after the resonator.

Figure 4.32: Close-in spectrum of LO oscillator output.

Figure 4.33: Close-in spectrum of RF oscillator output. .74

Figure 4.34: Phase noise measurement of down-converted RF signal. .75

Figure 4.35: Phase noise measurement of down-converted signal of oscillators using DB010485 amplifiers as active devices. .76

Figure 4.36: Phase noise comparison of commercially available, low noise, high performance oscillators, ultra-low-noise STALO and the oscillator designed in this thesis. .... 


\section{List of Tables}

Table 2-1: Noise processes used to describe phase noise in oscillators..........................................18

Table 2-2: Noise processes and power-laws of phase and frequency noise.....................................19

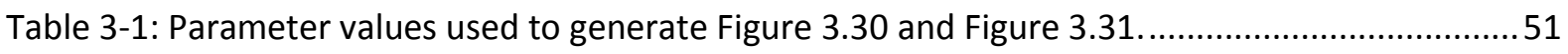

Table 4-1: Equipment used for the measurements performed in this section. ..................................54

Table 4-2: Simulated and measured loaded Q-factor of the resonator at its lowest-, centre-

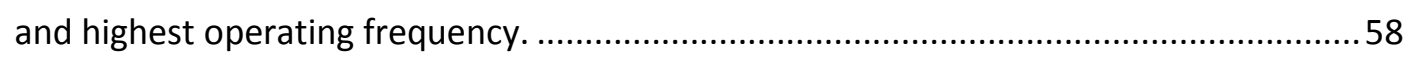

Table 4-3: Theoretical and measured loaded Q-factors of the resonator, with and without silver plating.

Table 4-4: Insertion loss and bandwidth of filter tuned to the lowest, highest and centre frequency.

Table 4-5: SSB phase noise power at various offset frequencies. 73

Table 4-6: SSB phase noise power at various offset frequencies with the $3 \mathrm{~dB}$ correction. .76

Table 4-7: SSB phase noise power of oscillator, with DB01-0485 amplifier as active device, at various offset frequencies.

Table 4-8: Phase noise performances of oscillators that will be used to compare to the oscillator designed in this thesis

Table 4-9: Comparison of commercially available high performance oscillators and ultra-lownoise STALO with oscillator designed in this thesis, translated to $9.2 \mathrm{GHz}$ operating frequency. 


\section{Nomenclature}

AGC

MWO

TE

TM

TEM

RBW

EFC

CST

FFT

IF
Automatic Gain Control

Microwave Office

Transverse Electric

Transverse Magnetic

Transverse Electromagnetic

Resolution bandwidth

Electronic Frequency Control

Computer Simulation Technology

Fast Fourier Transform

Intermediate Frequency 


\section{List of Appendices}

Appendix A CAD drawings - Resonator

Appendix B

CAD drawings - Filter

A-6

Appendix C

Datasheet - Narda DB01-0485 low noise amplifier

A-10

Appendix D

Datasheet - HMC-C200 DRO

A-12

Appendix E

Datasheet - PSI DRO-10.600-FR

A-19 


\section{Chapter 1}

\section{Introduction}

Oscillators are essential building blocks in wireless transmitter and receiver systems and serve as frequency references [1]. The quality of an oscillator is determined by the stability of its frequency. Phase noise performance is a measure of the frequency stability of an oscillator [2]. Phase noise can be seen in the time domain as fluctuations in the period of the signal's zero crossings. Increasing the operating frequency results in a degradation of phase noise performance [1]. Phase noise performance of an oscillator is largely determined by the loaded quality factor ( $Q$-factor) of the frequency selective element in the oscillator loop, the resonator. Thus phase noise performance can be improved by increasing the $\mathrm{Q}$-factor of the resonator [3].

\subsection{Problem statement}

The purpose of this thesis is to design and measure a low phase noise oscillator operating in X-band, at specifically $9.2 \mathrm{GHz}$. Phase noise performance must be investigated when the output signal is measured at different locations in the oscillator loop. A cylindrical cavity resonator must be used as the frequency selective element in the oscillator loop.

\subsection{Proposed solution}

The $\mathrm{TE}_{011}$ cylindrical waveguide mode has a higher unloaded $\mathrm{Q}$-factor than the dominant $\mathrm{TE}_{111}$ mode, but is in degeneracy with the $\mathrm{TM}_{111}$ mode. Tolerances in manufacturing can result in a different operating frequency than the design frequency, making it necessary to tune the operating frequency of the resonator. A perturbation made in a waveguide cavity structure that will serve as a tuning element can result in a lowering of the $\mathrm{Q}$-factor. The frequency of the $\mathrm{TE}_{011}$ mode can be tuned by inserting a tuning screw at one of the endplates of the cavity, extending in the axial direction. The loaded Q-factor will not be significantly influenced by the screw due to the circular direction of the surface currents on the endplates, i.e. no currents will flow in the axial direction on the screw and through the contact with the cavity endplate. The screw will also separate the degenerate $\mathrm{TM}_{111}$ and $\mathrm{TE}_{011}$ modes.

Critical coupling to the resonator will result in the highest loaded Q-factor, but will increase the insertion loss and in turn degrade phase noise performance [4]. An optimum coupling coefficient must be found in order for the resonator to minimise the phase noise of the oscillator.

Modes other than the desired mode will be excited and coupled out. If these modes are not suppressed, the oscillator may not operate at the desired frequency in the desired high Q mode of the resonator. A filter can be inserted into the oscillator loop to suppress spurious modes and ensure oscillation at the desired frequency. 
Low noise oscillator design techniques, as described in [5], will be used in order to ensure the best phase noise performance.

\subsection{Overview of thesis}

Chapter 2 serves as an introduction to oscillators. Basic terminology and design considerations are discussed. Noise inherent in oscillators and techniques to minimise it are described. Widely used microwave oscillator configurations and types of resonators are addressed. The last section of the chapter is dedicated to more complex configurations and techniques that are used to further minimise phase noise in oscillators, but these techniques are not incorporated in the oscillator considered in this thesis.

Chapter 3 explains the choice of components and the complete design thereof. Two components are designed; a resonator and a filter. The designs include simulations performed in CST and MATLAB.

Chapter 4 contains the necessary measurements that were done on the individual components and on the system as a whole. Measurements were performed by a vector network analyser (VNA), a spectrum analyser and a signal source analyser. The oscillator's phase noise performance was measured using different oscillator configurations. The phase noise performance of the oscillator designed in this thesis is compared to the phase noise performance of two high performance commercially available low noise oscillators whose components are not cryogenically cooled and an ultra-low-noise STALO.

Chapter 5 draws conclusions on the results and adds recommendations to further improve results. 


\section{Chapter 2}

\section{Oscillator Theory}

\subsection{Fundamentals}

In this section, the fundamental operation of an oscillator will be investigated and explained briefly. In short, an oscillator is a system that produces a periodic time varying voltage at the output, without receiving a time varying input signal [6].

The operation of an oscillator can be described by the linear feedback model as depicted in Figure 2.1 [7]. An oscillator inherently consists of an active device (amplifier), a frequency selective element (resonator) and an amplitude limiting element.

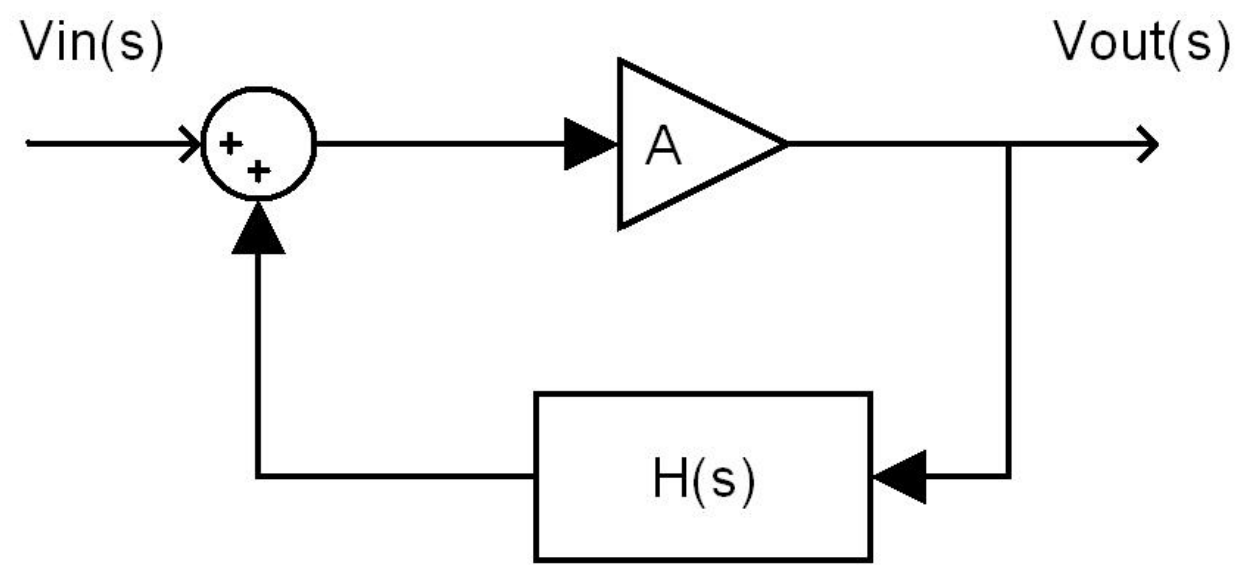

Figure 2.1: Block diagram of oscillator using amplifier and frequency dependent element in feedback path.

Equation (2.1) follows directly from Figure 2.1 and describes the output of the system as

$$
V_{\text {out }}(s)=A V_{\text {in }}(s)+H(s) A V_{\text {out }}(s)
$$

which simplifies to

$$
V_{\text {out }}(s)=\frac{A}{1-A H(s)} V_{\text {in }}(s)
$$

where $A$ is the gain of the amplifier and $H(s)$ is the frequency dependent transfer function of the frequency selective element [1]. As can be seen from (2.2), if the denominator becomes zero, it is possible to obtain an output without introducing an input to the system (except for biasing the active device with a DC voltage). 


\section{Barkhausen criteria}

In order for an oscillator to oscillate, it must satisfy the following conditions known as the Barkhausen criteria [7].

- At the loop closure point, the phase shift of the signal being fed back must be exactly zero degrees with respect to the input signal

- The overall steady state gain of the loop must be exactly unity at the loop closure point.

Both of these conditions must be satisfied to ensure steady state oscillation. In short, the system will oscillate at a frequency where the open-loop gain is equal or larger than unity with a phase shift of exactly zero degrees with respect to the input.

\section{Startup}

An oscillator relies on noise or a switch-on transient to start up. Noise that enters the active device (amplifier) is amplified. The frequency selective element only lets through a small band of frequencies (the bandwidth depends on the $Q$ of the resonator) and attenuates the rest. This signal is then fed back to the input of the active device, where it is amplified again and again until the active device saturates and the loop reaches a gain of exactly unity due to gain compression of the active device. It is important to note that the loop gain must be larger than unity at startup, otherwise oscillation will not start [8].

\section{Oscillation at other frequencies}

It is possible for the system to oscillate at frequencies other than the designed frequency. This occurs if there is another frequency where the loop gain is greater than unity with a phase shift of zero degrees. As long as the gain is less than unity at the frequency where the loop gain phase is zero degrees, the system will not oscillate at the undesired frequency [3].

\section{Maximum phase slope}

To achieve the best performance, with regard to long- and short term stability, the loop gain phase slope must be maximised at the phase zero crossing [3]. If the slope of the phase is very steep at the zero crossing, a change in loop gain phase will result in a small change in frequency.

\section{Excess gain and harmonics}

Referring to the Barkhausen criteria, the open-loop gain of the system can (and in most cases will) exceed unity at the phase zero crossing. This is referred to as excess loop gain [3]. If excess gain is present and the loop is closed by connecting the output to the input, the active device will become saturated and will limit the loop gain to unity. This limiting causes harmonics of the fundamental frequency to appear and degrades system performance. It is thus necessary to ensure that the loop gain stays as close to unity as possible. Care must still be taken to ensure that the loop gain does not fall short of unity (through parameter variations, temperature fluctuations etc.), as this might cause oscillations to die out. Another way to prevent limiting and ensure unity loop gain is to use an automatic gain control (AGC) in the circuit. An AGC controls the bias conditions of the active device which in turn adjusts the gain of the loop to stay close to unity while ensuring oscillation [3]. 


\subsection{Microwave and RF oscillators}

Two methods by which oscillators can be designed and analysed are described below. The first method involves looking into the oscillator in an open-loop configuration and analysing the magnitude and phase through the open-loop. Simple LC resonators contribute a zero degree phase shift at resonance, where the amplifier usually shows a 180 degrees phase shift. It is thus easy to manage the phase to meet the Barkhausen criteria.

The second method models the oscillator as a resonator connected to a one port negative resistance device.

Figure 2.2 illustrates the oscillator that will be used to explain these two methods.

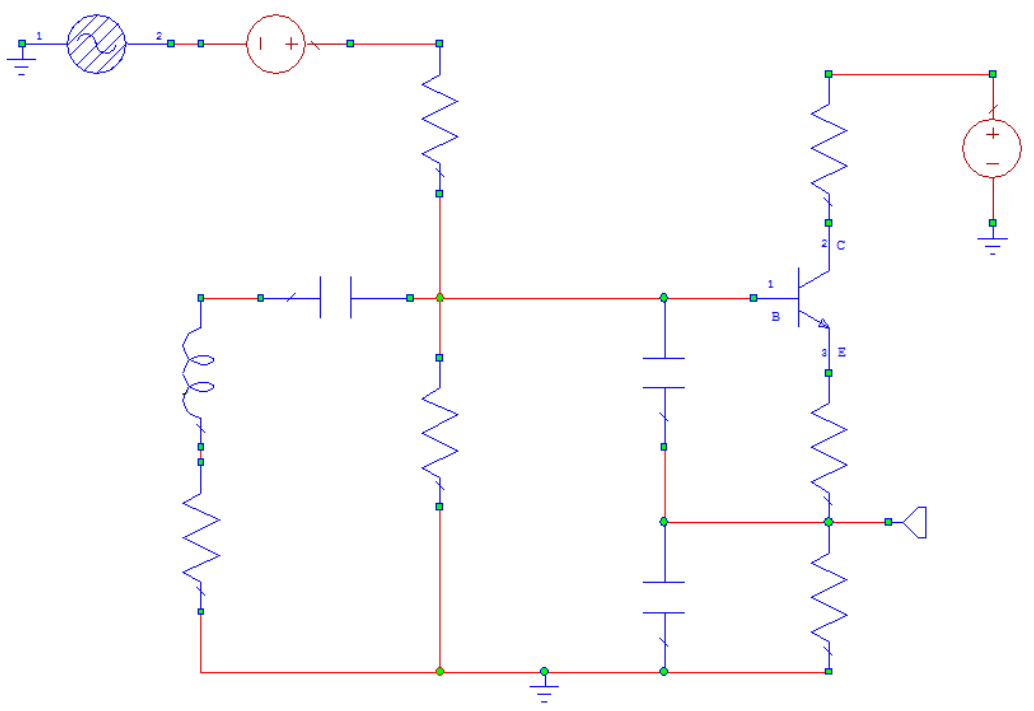

Figure 2.2: Schematic of oscillator that will be used in the following design methods.

\subsubsection{Method 1: Open-loop gain and phase}

To analyse the circuit with this method, the loop is broken as shown in Figure 2.3. To meet the Barkhausen criteria and thus ensure oscillation, the phase shift around the loop must be exactly zero degrees and the gain must be larger than unity at this frequency. The two ports that are formed by breaking the loop must be loaded with the impedances that they were loaded with before the loop was broken. 


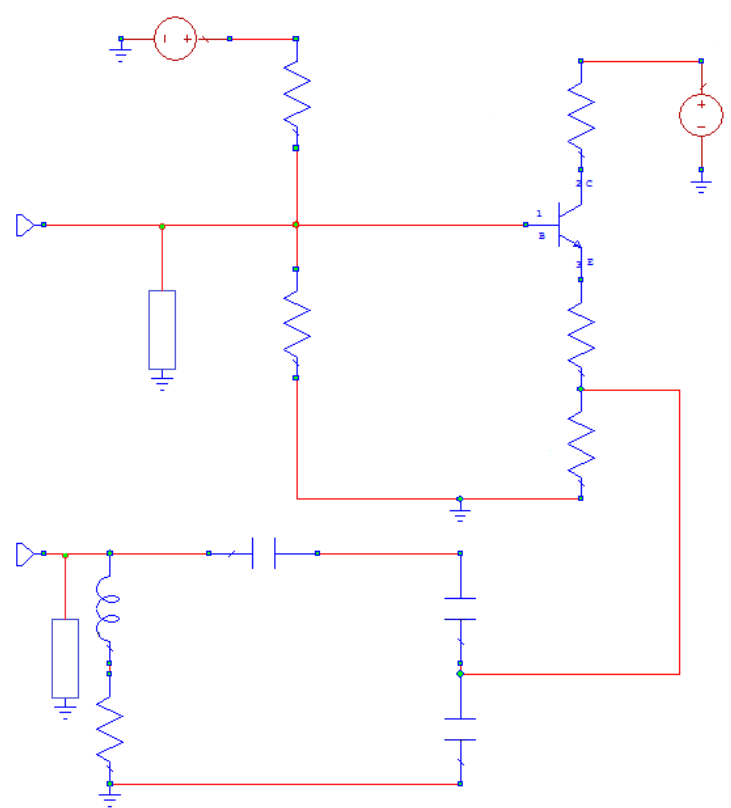

Figure 2.3: Open-loop oscillator used to meet the Barkhausen criteria using MWO.

The response obtained by MWO for this example is shown in Figure 2.4. It can be seen that the gain is greater than unity at the frequency where the phase shift is equal to zero. According to this analysis, the frequency of oscillation is $10.13 \mathrm{MHz}$.

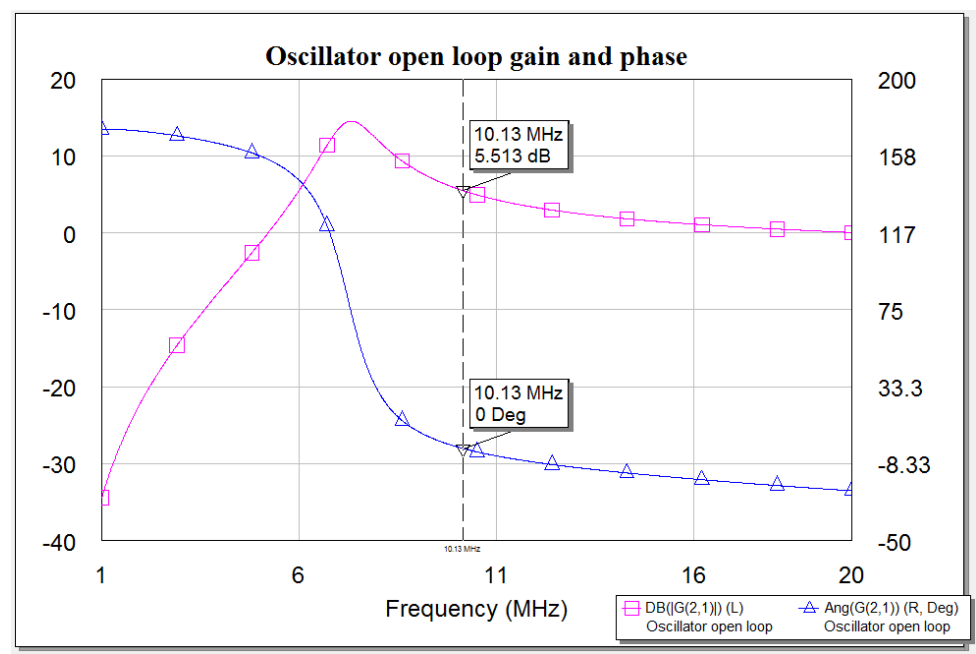

Figure 2.4: Simulation response of Figure 2.3 obtained from MWO. The circuit will oscillate where the phase is exactly zero degrees while the gain is larger than unity.

\subsubsection{Method 2: One-port negative-resistance device method}

In order for an oscillator to sustain oscillation, the active device must overcome the losses encountered in the loop to meet the Barkhausen gain criterion. A positive resistance represents a dissipation of energy, while a negative resistance represents an energy source. By making the active device unstable, a negative resistance is seen when looking into the input, while a positive resistance is seen when looking into the resonator. This negative resistance model, obtained from [3], is illustrated in Figure 2.5. 


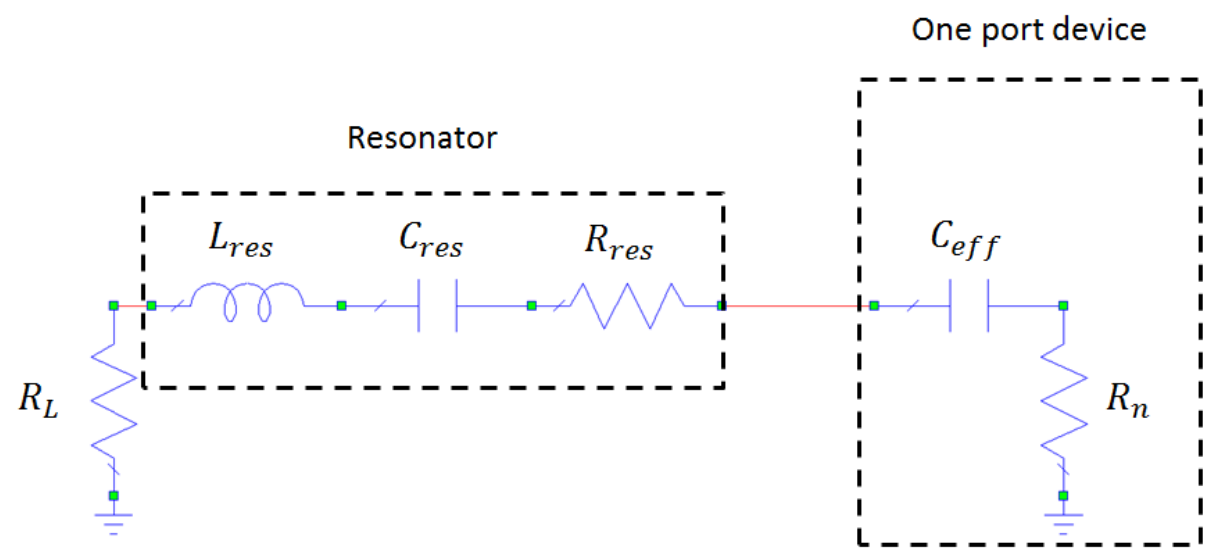

Figure 2.5: Negative resistance oscillator model.

Defining $Z_{L}$ as the combination of the impedance of the load and the resonator and $Z_{\text {in }}$ as the impedance of the active device and writing a KVL around the loop results in the following expression.

$$
\left(Z_{L}+Z_{\text {in }}\right) I=0
$$

If the circuit is oscillating, the current will be non-zero, which means that

$$
\begin{aligned}
& R_{\text {tot }}=R_{L}+R_{\text {in }}=0 \\
& X_{\text {tot }}=X_{L}+X_{\text {in }}=0
\end{aligned}
$$

The resonator and load are passive devices and thus have positive real parts, indicating that the real part of the one-port device must be negative to satisfy (2.5).

Analysing this circuit involves looking into the input ports of the one-port active device and the resonator, disconnected from each other, as shown in Figure 2.6. In this example a negativeresistance one-port device is created by making a transistor unstable. 


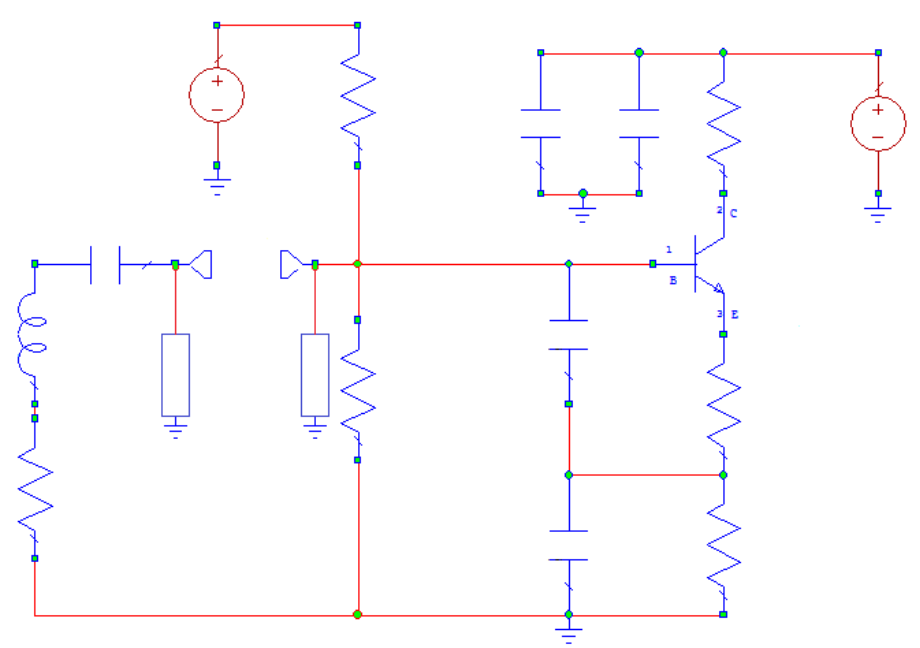

Figure 2.6: Circuit used for method 2. It involves looking into the input ports of the amplifier and resonator, separately, to obtain the frequency where the imaginary parts of the two circuits cancel each other while their resulting real part is negative. This will be the frequency of oscillation.

For the circuit to oscillate, the total reactance of the resonator and the one port device must be zero at a frequency where the total sum of the real parts of the resonator and one port device are negative. Figure 2.7 shows a simulation done in MWO by using the circuit in Figure 2.6. The graph indicates that the circuit will oscillate at $12.59 \mathrm{MHz}$.

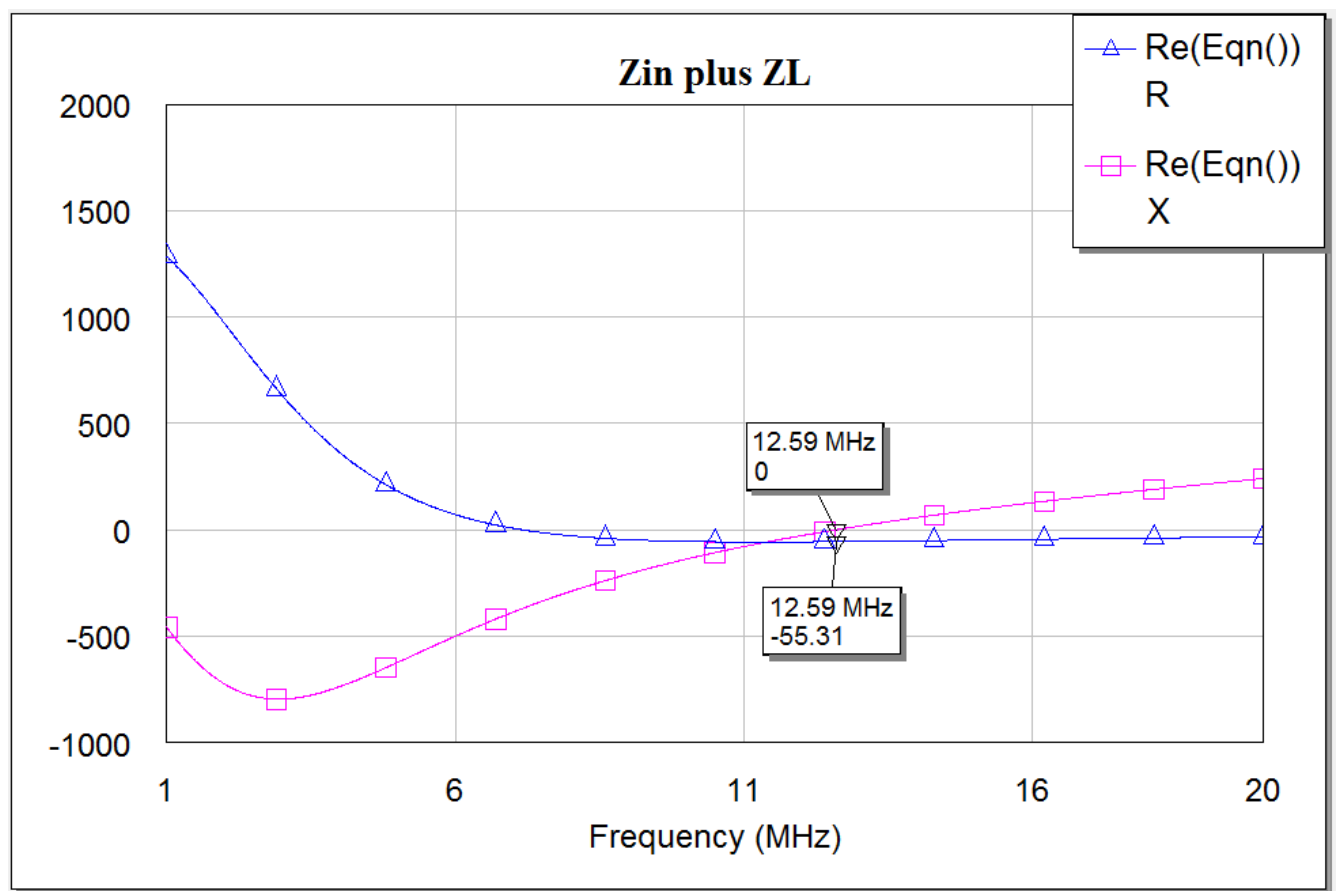

Figure 2.7: $\boldsymbol{R}_{\text {tot }}$ and $X_{\text {tot }}$ vs. frequency obtained from MWO. It indicates that the circuit will oscillate at 12.59 $\mathrm{MHz}$. 


\subsection{General oscillator elements}

An oscillator generally consists of a resonator, amplifier, coupler and phase shifter. Such an oscillator is shown in Figure 2.8 .

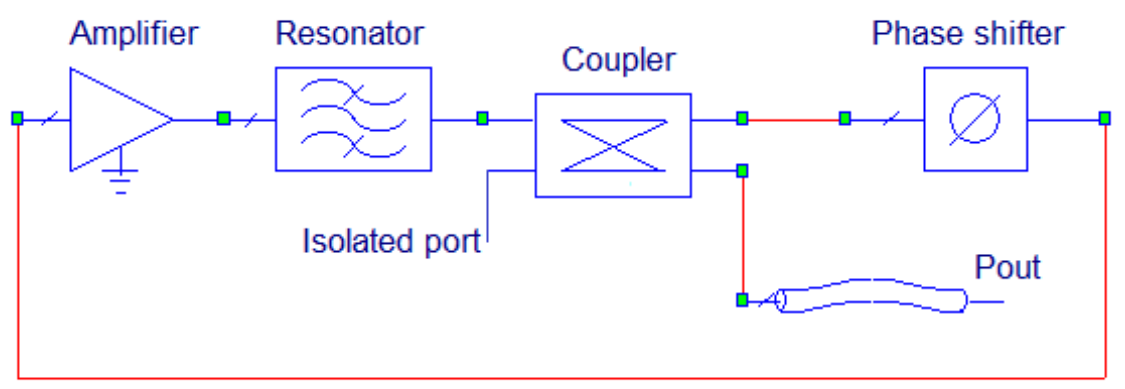

Figure 2.8: General elements forming an oscillator.

\section{Resonator}

The resonator is the frequency selective device in the oscillator loop. It is the most important component regarding phase noise, as it has the largest contributing factor in the phase noise curve, which will be described in section 2.7.2.

Many forms of microwave resonators exist which include loaded and unloaded cavities, dielectric resonators and transmission line resonators. Each is preferred for different applications. When a microwave resonator is near resonance, it can be modelled by either a series or parallel RLC lumped element circuit, as illustrated in Figure 2.9 (a) and Figure 2.9 (b), respectively [6]. Resonance occurs at the frequency where the total reactance or susceptance is zero and consequently the input impedance is real. An ideal resonator is lossless.

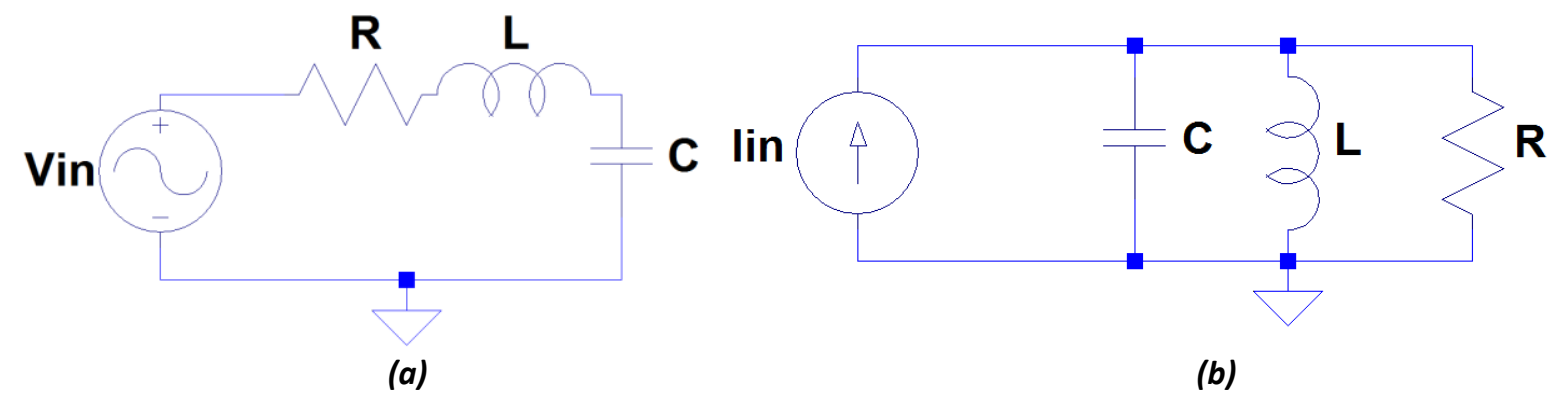

Figure 2.9: Model of microwave resonator near resonance in a (a) series configuration and (b) parallel configuration.

The resonant frequency for both the parallel and series circuits are given by [6]

$$
f_{r}=\frac{1}{2 \pi \sqrt{L C}}
$$




\section{Amplifier}

An amplifier is used generate signal power and to overcome the losses encountered in an oscillator loop and thus to ensure that the gain margin is larger than unity. In other words, it is used to startup and to sustain oscillation. In a low phase noise design, it is important to use an amplifier with a low noise figure.

\section{Coupler}

In a high frequency oscillator, a coupler is commonly used to couple energy out from the oscillator to an external load. At lower frequencies, an inductor or capacitor can be used to couple out energy. The energy can be coupled out anywhere in the loop, but better harmonic performance is obtained when the output is taken directly after the frequency selective element [3]. If the system has a high gain margin, a coupler with a large coupling value can be used and the loop can be closed through the coupled port, thus taking the output from the through port [3]. This will improve the harmonic performance and a large output power can be obtained.

\section{Phase shifter}

A phase shifter is inserted into the oscillator loop to adjust the loop gain phase to the optimal value for phase noise. Phase shifters can be electrically, magnetically or mechanically tuned, can be active or passive and can be digital or analogue [9].

\subsection{Types of high Q-factor microwave resonators and their}

\section{applications}

Oscillators are often named after the type of resonator used as the frequency selective element in the oscillator loop [5]. Some of the more popular resonators are described below.

\section{Distributed transmission line resonator}

The most general transmission line resonators are designed by using short-circuited and opencircuited half wavelength sections of transmission line and short-circuited quarter wave sections of transmission line when the electrical length of the line is a quarter wavelength or a half wavelength at the desired operating frequency [6]. Other types of distributed transmission line resonators also exist, but will not be discussed here.

\section{Cavity resonator}

A cavity can be constructed by placing endplates at the ends of a waveguide (rectangular or cylindrical), which forms a resonator [6]. The resonant frequencies of these cavities are discussed in Chapter 3 .

\section{Dielectric resonators}

A resonator can also be constructed by using a low-loss, high dielectric constant material in the shape of a small disc or cube [6]. The calculation of the resonant frequency of this type of structure 
is not trivial and will not be discussed here. The resonator is usually coupled to the oscillator by placing it close to a microstrip line, where it couples to the magnetic fringing of the microstrip line [6]. Cavity resonators can also be loaded with dielectric resonators to reduce losses.

\section{YIG resonators}

A resonator is formed by using a ferrite sphere of yttrium iron garnet. The operating frequency can be tuned over a wide band by changing the biasing dc magnetic field [5].

\section{Applications}

In high power applications, a cavity resonator is preferred due to the metallic structure's high power handling capability. Should the resonator be used in a physically small circuit, dielectric resonators are often preferred due to its light weight and small size. Transmission line resonators generally have the same applications as dielectric resonators, but suffer from a low Q-factor. YIG resonators can be used in applications where a wideband tuneable oscillator is needed.

\subsection{Resonator losses and the Q-factor}

An important parameter of a resonator is its Q-factor, defined by [6] as

$$
Q=\omega_{r} \frac{\text { average energy stored }}{\text { energy loss } / \text { second }}
$$

where $\omega_{r}$ is the resonant frequency. The Q-factor is a convenient figure of merit to quantify resonator losses. The loaded, external and unloaded $\mathrm{Q}$-factors are related by the following equation as

$$
\frac{1}{Q_{l}}=\frac{1}{Q_{e x}}+\frac{1}{Q_{0}}
$$

where $Q_{l}, Q_{e x}$ and $Q_{0}$ are the loaded, external and unloaded Q-factors, respectively [10]. When the resonator is connected to external circuitry, for instance a signal source and load, one obtains $Q_{l}$. The unloaded Q-factor is determined when no external circuitry is connected to the resonator and the external Q-factor is determined by the loading of the resonator [10]. The resonator coupling factor is defined as [10] "the ratio of power dissipated in the external circuit to the power dissipated in the resonator is called the coupling coefficient $k$ ".

$$
\kappa=\frac{Q_{0}}{Q_{e x}}
$$

The loaded Q of a resonator is calculated as

$$
Q_{l}=\frac{f_{r}}{3 d B B W}
$$


where the denominator represents the half-power bandwidth of the resonator impedance, admittance or transfer function and $f_{r}$ is the resonant frequency. Figure 2.10 illustrates the amplitude response of the immittance of a simple resonator vs. frequency, clearly indicating the $3 \mathrm{~dB}$ bandwidth.

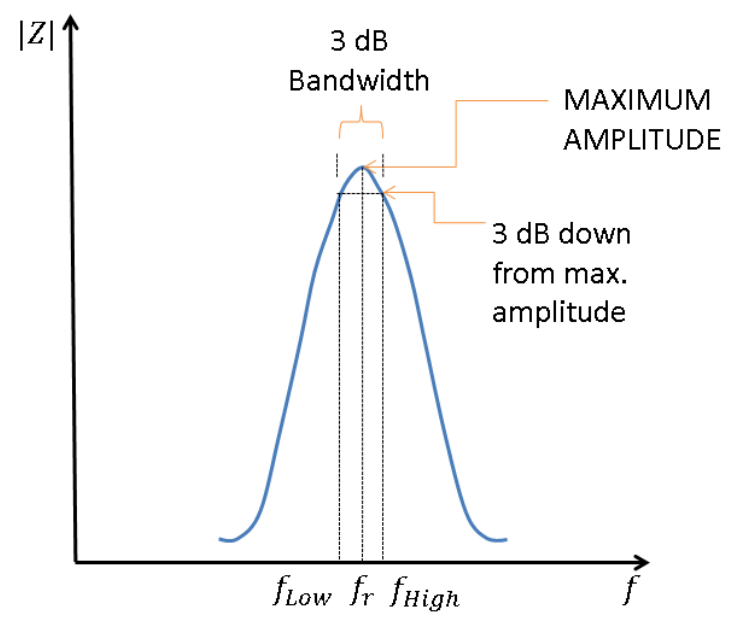

Figure 2.10: Passband characteristics of resonator. Upper and lower frequency used to calculate half power bandwidth is determined by obtaining the frequencies where the power is $3 \mathrm{~dB}$ down from the maximum transmission power at the centre frequency.

$Q_{l}$ can also be determined from the group delay, where the group delay is equal to the phase slope of the relevant response [3]

$$
t_{d}(\omega)=\frac{-d \varphi}{d \omega}
$$

The loaded Q-factor can then be calculated from the group delay of the impedance or admittance function as [3]

$$
Q_{l}=\frac{\omega_{r} t_{d r}}{2}
$$

where $\omega_{r}$ is the resonant frequency in rad. $s^{-1}$ and

$$
t_{d r}=t_{d}\left(\omega_{r}\right)
$$

Alternatively, the loaded Q-factor can be calculated as

$$
Q_{l}=\pi f_{r} t_{d r}
$$

where

$$
f_{r}=\frac{\omega_{r}}{2 \pi}
$$


A maximum phase slope at the phase zero crossing is an important parameter in oscillators. Since the Q-factor of a resonator is a function of the phase slope, it can be seen why the Q-factor is such an important parameter. In an oscillator the phase slope should be maximised at the resonant frequency. The phase shifter in the loop is used to adjust the operating point the optimal value.

Rhea [3] suggests that the loaded Q-factor should be calculated by using the group delay method when the maximum phase slope does not occur at the phase zero crossing, otherwise the amplitude response bandwidth method can be used by measuring the loop gain.

\subsection{Noise in oscillators}

The output of an ideal oscillator can be described by a sinusoidal function

$$
V(t)=V_{0} \sin \left(2 \pi v_{0} t+\varphi\right)
$$

where $V_{0}$ and $v_{0}$ represent the nominal amplitude [V] and frequency $[\mathrm{Hz}]$ and $\varphi$ is an arbitrary phase constant that can be set to zero. This maps to the Dirac delta function in the frequency domain [11], [12].

$$
V(f)=\frac{j}{2}\left[\delta\left(f+f_{0}\right)-\delta\left(f-f_{0}\right)\right]
$$

In reality an oscillator's output is never perfectly sinusoidal. It contains amplitude, frequency and phase fluctuations. Thus, a realistic oscillator's output can be described as

$$
V(t)=\left[V_{0}+\varepsilon(t)\right] \sin \left[2 \pi v_{0} t+\phi(t)\right]
$$

where $\varepsilon(t)$ and $\phi(t)$ represent amplitude and phase fluctuations [2], respectively. According to [13], fluctuation in the period of oscillation results in frequency instability, fluctuations in the phase results in jitter (the term used to describe fluctuations in the period of the signal's zero crossings) and fluctuations in the peak value of the signal results in amplitude noise.

These fluctuations cause the spectrum of the oscillator's output to widen. A typical spectrum of an oscillator's output is shown in Figure 2.11. 


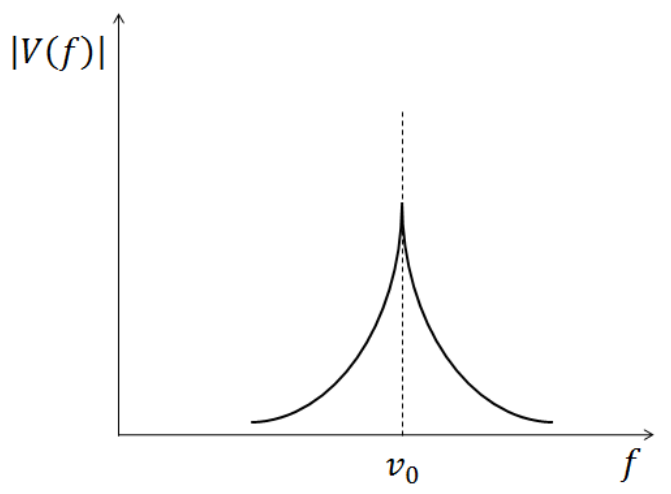

Figure 2.11: Typical spectrum of oscillator output.

To illustrate amplitude and phase noise more clearly, it is worth showing their individual contributions.

\subsubsection{Amplitude noise}

A sinusoid containing only amplitude noise is shown in Figure $2.12(\Phi(t)$ in $(2.18)$ is thus zero), where the noise is chosen as a single sinusoid of lower frequency in order to illustrate the process more clearly. Also shown is the noiseless signal.

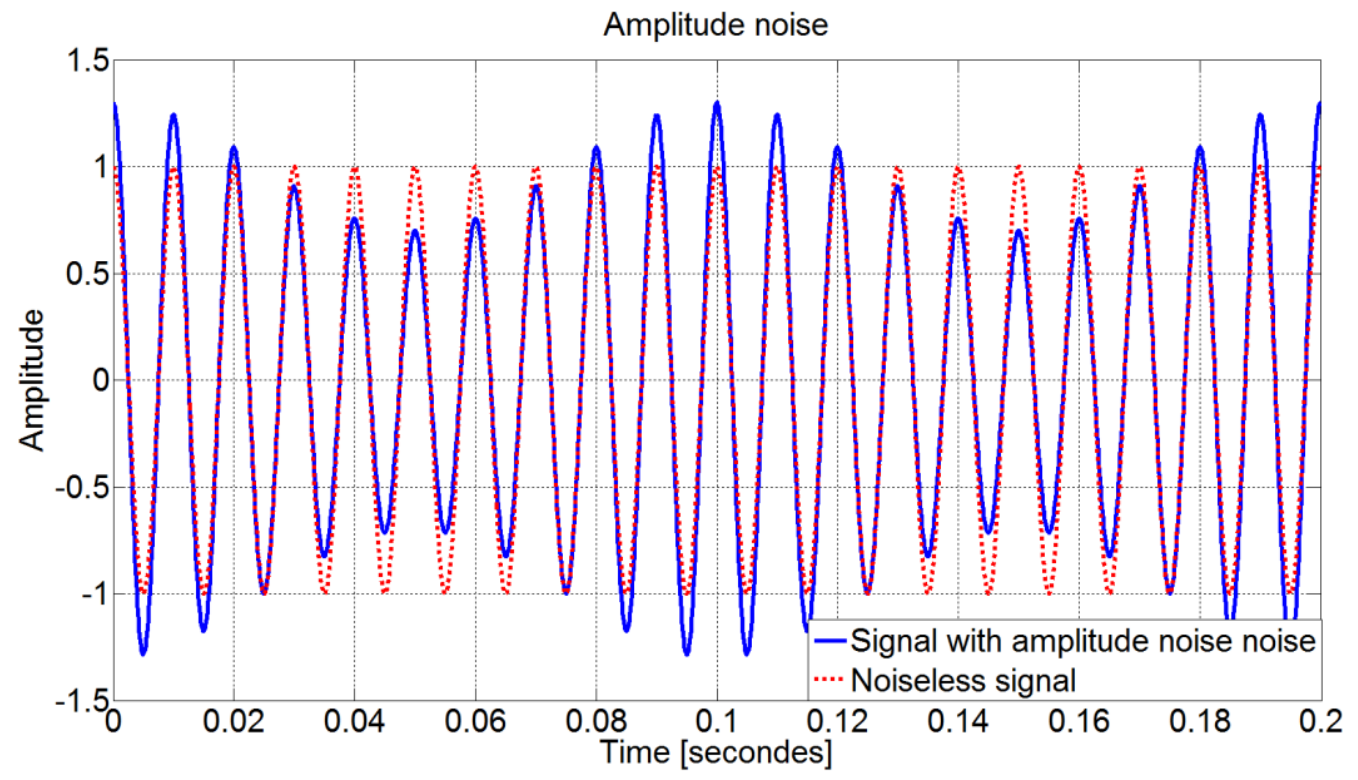

Figure 2.12: Signal containing only amplitude noise.

The noiseless and noisy signals are given in (2.19) and (2.20), respectively as

$$
\begin{gathered}
V(t)=V_{0} \cos \left(2 \pi v_{0} t\right) \\
V(t)=V_{0}(1+A(t)) \cos \left(2 \pi v_{0} t\right)
\end{gathered}
$$

where 


$$
A(t)=\alpha \cos \left(2 \pi f_{m} t\right)
$$

in this example. Also, $\mathrm{V}_{0}$ is chosen as unity, $\alpha$ is the amplitude modulation index and $f_{m}$ is the amplitude modulation frequency. Substituting (2.21) into (2.20) and simplifying, yields

$$
V(t)=\cos 2 \pi v_{0} t+\frac{\alpha}{2}\left(\cos \left(2 \pi v_{0}-2 \pi f_{m}\right) t+\cos \left(2 \pi v_{0}+2 \pi f_{m}\right) t\right)
$$

Viewing this signal in the frequency domain, it is clear that the noise signal is amplitude modulated by the carrier signal and results in sidebands around the carrier. Figure 2.13 (a) shows the power spectrum to illustrate this process.

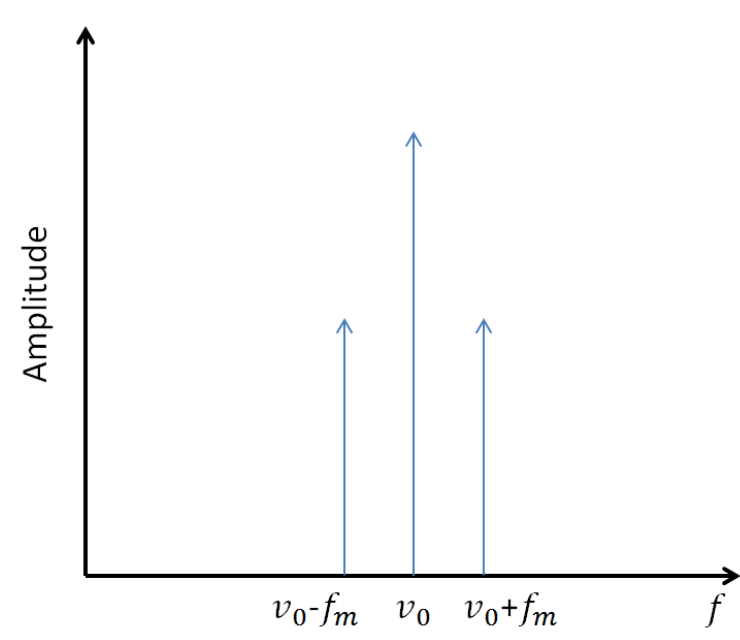

(a)

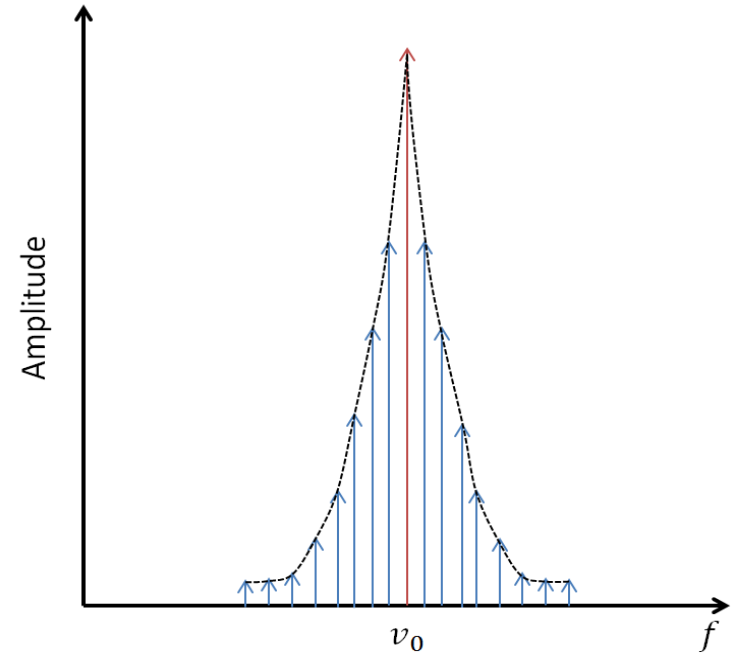

(b)

Figure 2.13: Signal amplitude modulated by $a(a)$ single sinusoid and (b) a concentration of random sinusoids.

The case explained above is a special case when the noise is a single sinusoid. Figure 2.13 (b) shows a more generalized discrete spectrum of a signal containing random amplitude noise where each measurement value represents the amplitude noise in a finite bandwidth.

\subsubsection{Phase noise}

Signals containing only phase noise and no amplitude noise will be discussed in this section. A sinusoid with phase noise can be written as

$$
V(t)=V_{0} \cos \left(2 \pi v_{0} t+\phi(t)\right)
$$

where

$$
\phi(t)=\tau \sin \left(2 \pi f_{m} t\right)
$$

represents the phase noise and $\tau$ represents the maximum phase deviation in this example. The phase noise is represented as a sinusoid of constant frequency. A noiseless and a noisy sinusoid are shown in Figure 2.14 (a). The noiseless signal has zero crossings at exact periodic time intervals. The 
time intervals of the noisy signal's zero crossings on the other hand are not periodic, as can be seen from the figure. These fluctuations in the time intervals of the zero crossings are referred to as phase noise.

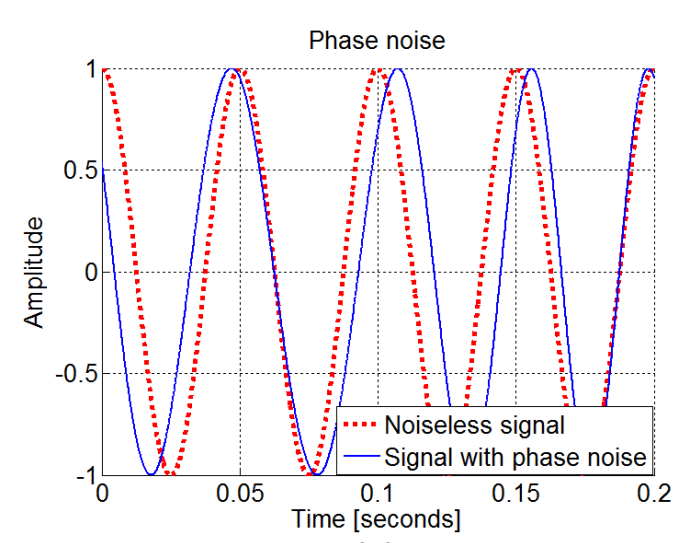

(a)

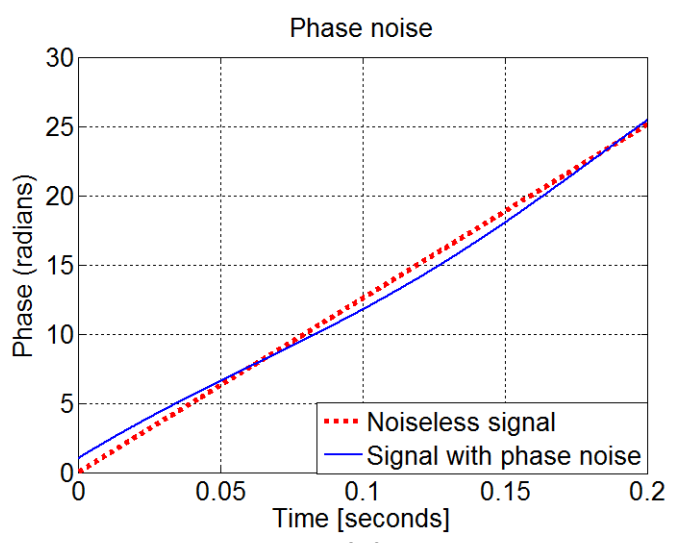

(b)

Figure 2.14: Time domain signal containing phase noise, illustrating the variation of its phase by looking at the (a) amplitude and (b) phase.

Figure 2.14 (b) shows the phase of the two signals vs. time. The instantaneous frequency of an arbitrary signal is determined by the rate of change of its phase, or

$$
f=\frac{d}{d t} \theta(t)
$$

where $\theta(t)$ represents the total phase of a signal. The instantaneous frequency of the noisy signal in the example can be written as

$$
f=2 \pi v_{0}+2 \pi f_{m} \tau \cos \left(2 \pi f_{m} t\right)
$$

The frequency of the noisy signal is not constant, but varies with time. The instantaneous frequency is embedded in the instantaneous phase, revealing that the variation in phase is indistinguishable from the variation in frequency.

Inserting (2.24) into (2.23) and writing it in a more usable form results in

$$
V(t)=V_{0}\left[\cos \left(2 \pi v_{0} t\right) \cos \left(\tau \sin \left(2 \pi f_{m} t\right)\right)-\sin \left(2 \pi v_{0} t\right) \sin \left(\tau \sin \left(2 \pi f_{m} t\right)\right)\right]
$$

Assuming that the maximum phase deviation is small so that $\tau \ll 1$, the approximation of small angles can be made without loss of information. This means that $\cos (\tau) \approx 1$ and $\sin (\tau) \approx \tau$. Making these approximations results in

$$
\begin{aligned}
V(t) & =V_{0}\left[\cos \left(2 \pi v_{0} t\right)-\tau \sin \left(2 \pi f_{m} t\right) \sin \left(2 \pi v_{0} t\right)\right] \\
& =V_{0}\left[\cos 2 \pi v_{0} t-\frac{\tau}{2}\left(\cos 2 \pi\left(v_{0}-f_{m}\right) t-\cos 2 \pi\left(v_{0}+f_{m}\right) t\right)\right]
\end{aligned}
$$

The above equation reveals that the signal consists of the carrier, accompanied with an upper and lower sideband. Also looking at the frequency spectrum of this signal reveals that phase noise 
exhibits the same characteristics as amplitude noise. Figure 2.15 (a) shows the spectrum of the signal used in the example above and Figure 2.15 (b) shows a more generalized discrete spectrum of a signal containing random phase noise where each measurement value represents the phase noise in a finite bandwidth. For theoretical purposes the measurement bandwidth is usually defined as 1 $\mathrm{Hz}$.

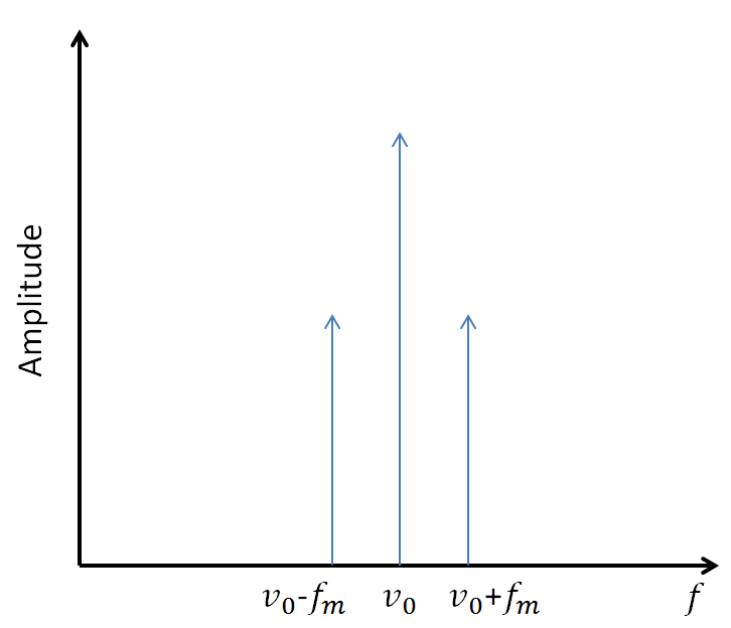

(a)

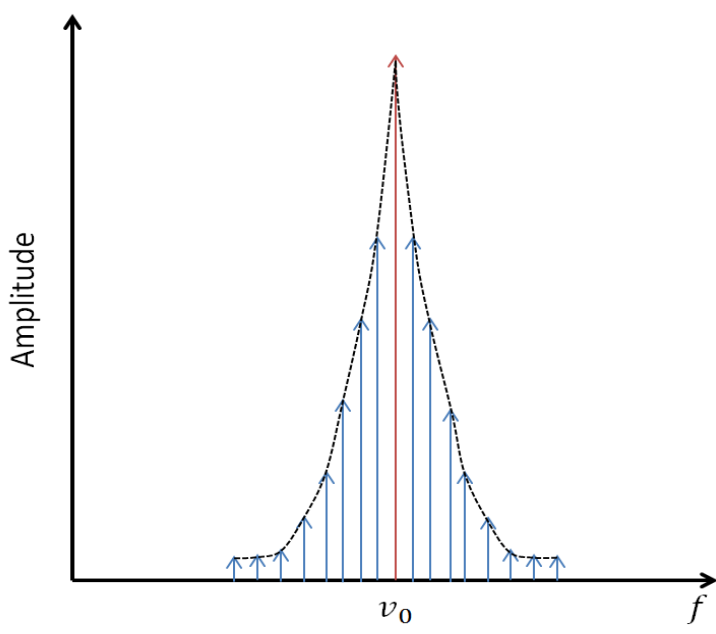

(b)

Figure 2.15: Signal phase modulated by a (a) single sinusoid and (b) a concentration of random sinusoids.

Note that the spectrum of the phase noise and the spectrum of the amplitude noise have the same appearance. It should be noted that although the spectra look the same, the magnitude of the amplitude noise spectrum is usually much smaller than the magnitude of the spectrum of the phase noise [14].

It now becomes clear that the phase noise of an oscillator cannot reliably be read off directly from a spectrum analyser, as it is impossible to separate the phase noise from the amplitude noise in this fashion.

\subsubsection{Characterization of phase noise}

The phase fluctuations $\phi(t)$ can be treated as a zero-mean stationary random process [15]. Noise generated in all electronic circuits include thermal noise (also known as Johnson- or Nyquist noise), shot noise and flicker noise. They are best described by their power spectral density (PSD) as [16]

$$
\begin{gathered}
S_{\text {thermal }}(f)=4 k T B R \\
S_{\text {shot }}(f)=2 q I B \\
S_{\text {flicker }}(f) \propto 1 / f
\end{gathered}
$$

where

- $\mathrm{k}=$ Boltzmann's constant, $1.38 \mathrm{e}-23[\mathrm{~J} / \mathrm{K}]$

- $\mathrm{T}=$ Temperature [K], (290 K at room temperature)

- $\mathrm{B}=$ Measuring bandwidth $[\mathrm{Hz}]$

- $\mathrm{R} \quad=$ Resistance $[\Omega]$ 
- $\mathrm{q}=$ Charge of an electron, 1.602e-19 [C]

- I = DC current $[\mathrm{A}]$.

Two categories of noise exist, namely ultimate noise and excess noise. Ultimate noise depends on the physics of the materials of the components and it sets a lower limit to the system's noise performance, while excess noise depends on the quality of the components. Thermal- and shot noise belong to the former category while flicker noise belongs to the latter [16].

One way to describe the spectral performance of an oscillator is the one-sided power spectral density of the phase noise, $S_{\phi}(f)$, with dimensions of $\left[\frac{\mathrm{rad}^{2}}{\mathrm{~Hz}}\right]$ [17]. Another way to describe oscillator phase noise is the quantity $\mathcal{L}(f)$, read "script el of $f$ ", and is related to $S_{\phi}(f)$ as

$$
\mathcal{L}(f)=\frac{1}{2} S_{\phi}(f)
$$

and is usually_expressed in decibels as $10 \log \mathcal{L}(f)$ with units of $(\mathrm{dBc} / \mathrm{Hz})$ [8]. $\mathcal{L}(f)$ is more commonly used among manufacturers and users of frequency standards and its units are decibels below the carrier in a $1 \mathrm{~Hz}$ bandwidth, or $\mathrm{dBc} / \mathrm{Hz}$ [13]. The IEEE standard 1139 [13] recommends that the $\mathcal{L}(f)$ notation should be used for describing the phase noise spectra in practice.

The IEEE standard 1139 [13] explains how to measure phase noise in the time domain by means of the fully overlapped Allan deviation $\sigma_{y}(\tau)$, but this will not be discussed here.

\subsubsection{Power-law phase noise distribution}

Table 2-1 describes the most significant noise processes encountered in oscillators. The power-law function, given in (2.30), describes the contribution of these various noise processes which yields the phase noise spectra of an oscillator. These processes are then plotted together on a log-log scale where the term $f^{i}$ indicates a straight line with a slope of i. Figure 2.16 illustrates the slope characteristics of these five noise processes [13].

Table 2-1: Noise processes used to describe phase noise in oscillators.

\begin{tabular}{|l|c|c|}
\hline Description of noise process & $\boldsymbol{i}$ & Law \\
\hline White phase modulation & 0 & $b_{0} f^{0}$ \\
\hline Flicker phase modulation & -1 & $b_{-1} f^{-1}$ \\
\hline White frequency modulation & -2 & $b_{-2} f^{-2}$ \\
\hline Flicker frequency modulation & -3 & $b_{-3} f^{-3}$ \\
\hline $\begin{array}{l}\text { Random walk frequency } \\
\text { modulation }\end{array}$ & -4 & $b_{-4} f^{-4}$ \\
\hline
\end{tabular}

$$
S_{\phi}(f)=\sum_{\substack{i=-4 \\ \text { or less })}}^{0} b_{i} f^{i}
$$




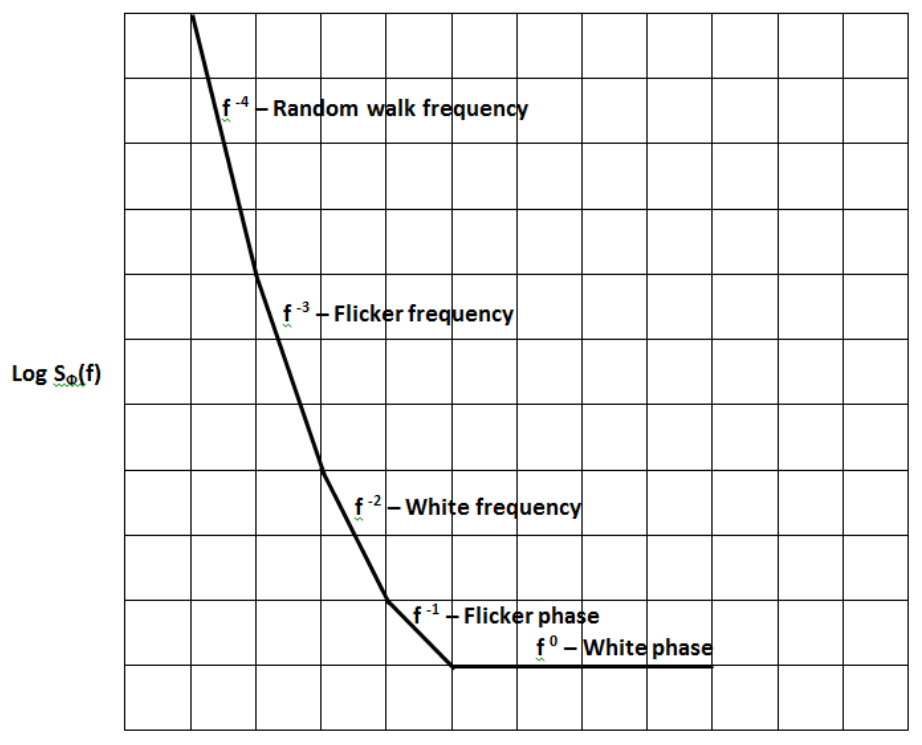

Log Offset frequency

Figure 2.16: Phase noise distribution according to the power-law model.

Oscillator noise can also be described in terms of its frequency fluctuations. The PSD of the frequency noise is given as

$$
S_{y}(f)=\frac{f^{2}}{v_{0}^{2}} S_{\phi}(f)
$$

The power-law model can also be used to describe the spectra of the frequency noise distribution as [8]

$$
S_{y}(f)=\sum_{i=-2}^{2} h_{i} f^{i}
$$

where

$$
h_{i}=\frac{1}{v_{0}^{2}} b_{i-2}
$$

Table 2-2 relates the noise processes and their power-laws of the phase noise and frequency noise PSDs. $S_{y}(f)$ is also plotted on a log-log scale in the same fashion as $S_{\phi}(f)$.

Table 2-2: Noise processes and power-laws of phase and frequency noise.

\begin{tabular}{|l|c|c|}
\hline Noise process & Phase noise PSD $\boldsymbol{S}_{\boldsymbol{\phi}}(\boldsymbol{f})$ & Frequency noise PSD $\boldsymbol{S}_{\boldsymbol{y}}(\boldsymbol{f})$ \\
\hline White phase modulation & $b_{0} f^{0}$ & $h_{2} f^{2}$ \\
\hline Flicker phase modulation & $b_{-1} f^{-1}$ & $h_{1} f^{1}$ \\
\hline White frequency modulation & $b_{-2} f^{-2}$ & $h_{0} f^{0}$ \\
\hline Flicker frequency modulation & $b_{-3} f^{-3}$ & $h_{-1} f^{-1}$ \\
\hline Random walk frequency modulation & $b_{-4} f^{-4}$ & $h_{-2} f^{-2}$ \\
\hline
\end{tabular}




\subsubsection{The Leeson formula for phase noise distribution}

In 1966 D.B. Leeson presented a model for short term frequency stability of an oscillator in a paper entitled, "A simple model of feedback oscillator noise spectrum" [15]. The Leeson formula (2.34) relates the PSD of the output phase noise of the oscillator to the input's phase noise PSD of the active device. The output and input PSDs are related as

$$
S_{\phi}(f)=\left[1+\frac{1}{f^{2}}\left(\frac{v_{0}}{2 Q_{L}}\right)^{2}\right] S_{\psi}(f)
$$

where

- $S_{\phi}(f)=$ Oscillator's output phase noise PSD

- $S_{\psi}(f)=$ PSD of phase noise generated by the active device

- $v_{0} \quad=$ Nominal frequency of oscillation

- $f \quad=$ Offset frequency from carrier

- $Q_{l} \quad=$ Loaded Q-factor of resonator

The Leeson frequency is defined as

$$
f_{L}=\frac{v_{0}}{2 Q_{l}}
$$

The output PSD of a linear system is equal to the input PSD times the magnitude of the transfer function squared; in this case the phase noise transfer function. Figure 2.17 illustrates the magnitude of the transfer function squared, plotted on a log-log scale.

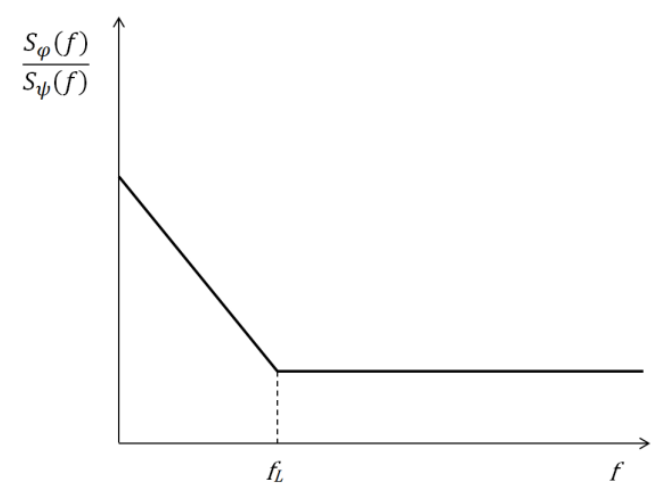

Figure 2.17: Transfer function relating the noise PSD at the input to the noise PSD at the output of an amplifier.

The input phase noise PSD at the amplifier can be expressed as [5]

$$
S_{\psi}(f)=\frac{F k T B}{P_{\text {in }}}\left(1+\frac{f_{c}}{f}\right)
$$

By inserting (2.36) into (2.34), the output phase noise PSD becomes 


$$
S_{\phi}(f)=\frac{k T F B}{P_{\text {in }}}\left[\frac{1}{f^{3}}\left(\frac{v_{0}}{2 Q_{L}}\right)^{2} f_{c}+\frac{1}{f^{2}}\left(\frac{v_{0}}{2 Q_{L}}\right)^{2}+\frac{1}{f} f_{c}+1\right]
$$

Referring to (2.29), the output phase noise of an oscillator is described by $\mathcal{L}(f)$ as

$$
\mathcal{L}(f)=10 \log _{10}\left(\frac{k T F B}{2 P_{\text {in }}}\left[\frac{1}{f^{3}}\left(\frac{v_{0}}{2 Q_{L}}\right)^{2} f_{c}+\frac{1}{f^{2}}\left(\frac{v_{0}}{2 Q_{L}}\right)^{2}+\frac{1}{f} f_{c}+1\right]\right)
$$

where

- $F \quad=$ Amplifier noise figure

- $P_{\text {in }} \quad=$ Input power to the amplifier

- $f_{c} \quad=$ Corner frequency of up-converted $1 / \mathrm{f}$ noise

Note that the bandwidth is taken as $1 \mathrm{~Hz}$ due to the definition of $\mathcal{L}(f)$. The four major causes of oscillator phase noise is contained in (2.38) and referring to the power-law model and Table 2-2, these noise processes (from left to right in the square brackets of (2.38)) are flicker FM, white FM, flicker phase and white phase noise.

Two types of output phase noise spectrums can be encountered, depending on whether the Leeson frequency is higher or lower than the flicker corner frequency of the active device. Usually the case $\mathrm{f}_{\mathrm{L}}>\mathrm{f}_{\mathrm{c}}$ is treated as a low $\mathrm{Q}$ resonator case and the case $\mathrm{f}_{\mathrm{L}}<\mathrm{f}_{\mathrm{c}}$ as a high $\mathrm{Q}$ resonator case. The two expected phase noise slope characteristics are illustrated in Figure 2.18 (a) and Figure 2.18 (b).

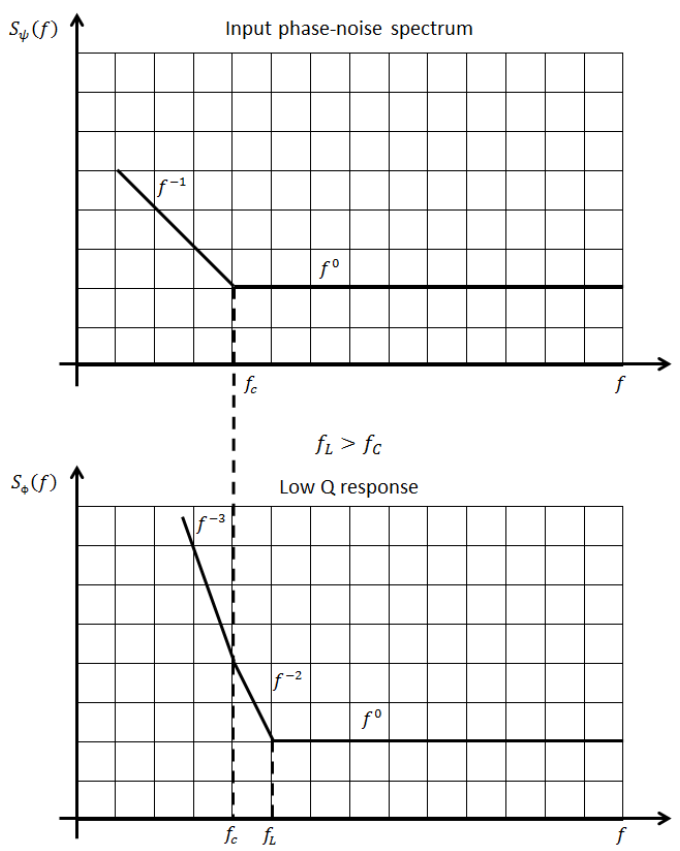

(a)

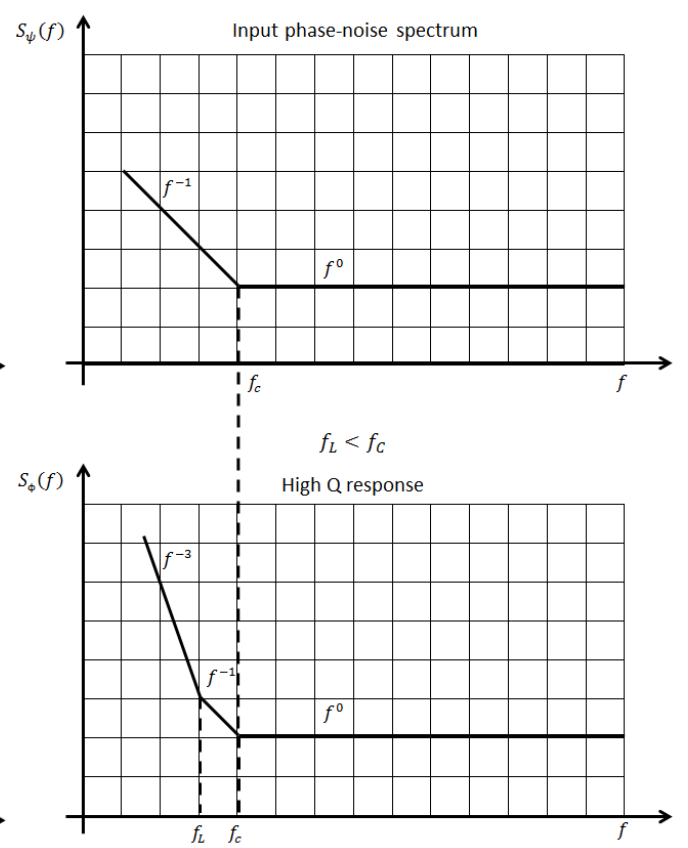

(b)

Figure 2.18: Phase noise distribution according to Leeson when $(a) f_{L}>f_{c}$ and (b) $f_{L}<f_{c}$.

The variables used in this section are in accordance with the IEEE 1139 standard [13]. 


\subsection{Configurations and techniques to reduce phase noise in}

\section{microwave oscillators}

The conventional methods to reduce phase noise in oscillators include [5]

- Using a high-Q resonator

- Choosing an active device with a low noise-figure

- Choosing an active device with low flicker-corner frequency

- Ensuring the open-loop gain of the oscillator is near unity

- Increasing the power level at the input of the active device

The methods described above will be used in the oscillator considered in this thesis. In the following section, oscillator configurations and techniques are briefly described by which phase noise can further be reduced [4], [18], [19], [20], [21]. These configurations and techniques, however, will not be incorporated in the oscillator described in this thesis. They are included to serve as a recommendation for designing more complex oscillators with better phase noise performance.

Consider the oscillator configuration shown in Figure 2.19. The configuration mainly consists of a voltage controlled oscillator (VCO), phase-locked to an external high-Q resonator tuned to the fundamental frequency of oscillation. These types of configurations are referred to as stabilized local oscillator (STALO) circuits. The phase noise of this configuration is effectively reduced by cancelling the frequency fluctuations of the VCO through detecting the resulting phase shift of the microwave signal through an external high- $\mathrm{Q}$ resonator and feeding a frequency correction voltage back to the VCO. The phase of the signals at the two mixer inputs are adjusted to be in quadrature in order to for the mixer to operate in its high slope region of output vs. phase and thus obtain maximum sensitivity. 


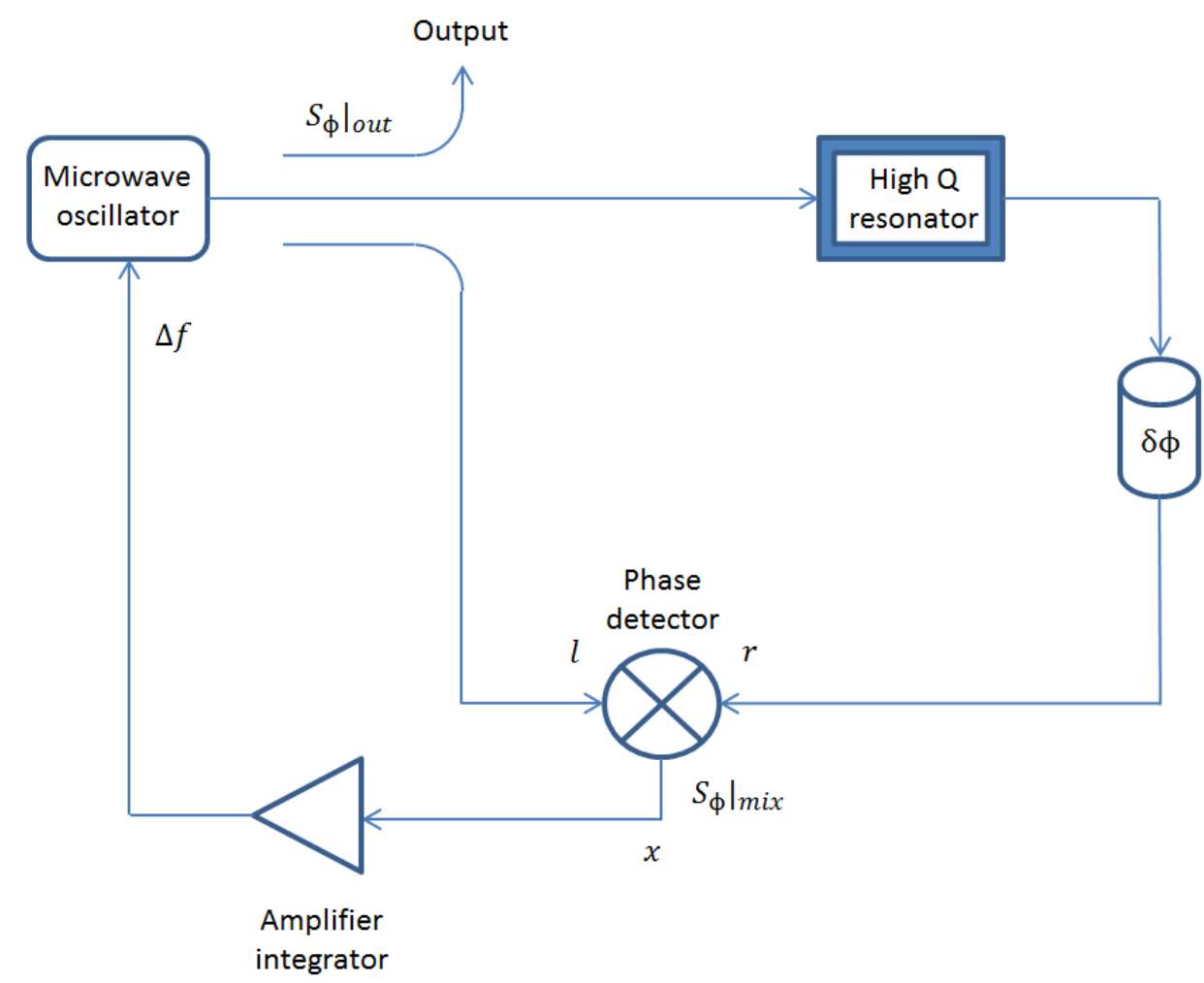

Figure 2.19: STALO consisting of a microwave oscillator, phase locked to a high Q resonator [18].

Consider a signal containing a frequency error at the output of the VCO; a portion of this signal is taken as an output, a portion is fed to the $l$ input-port of the mixer and a portion is coupled to the input of the high-Q resonator. Recall that the resonator's centre frequency is tuned to the fundamental frequency of oscillation. Referring to Figure 2.19, if a frequency error is present in the signal, the phase of the signal will be shifted accordingly. This phase-shifted signal is then fed to the other input arm of the mixer and its phase is compared to that of the original signal. The phase difference between the signals results in a frequency correction voltage determined by the feedback loop and the operating frequency of the VCO is corrected.

This configuration can further be improved by using a critically coupled one-port high-Q resonator, taking the reflected signal (if present due to frequency variations) and amplifying it before comparing it to the VCO signal. This configuration is shown in Figure 2.20. The improvement achieved in this configuration is by reducing the effect of the mixer's noise by the gain of the RF amplifier, and reducing this amplifier's multiplicative phase noise by suppressing the carrier. 


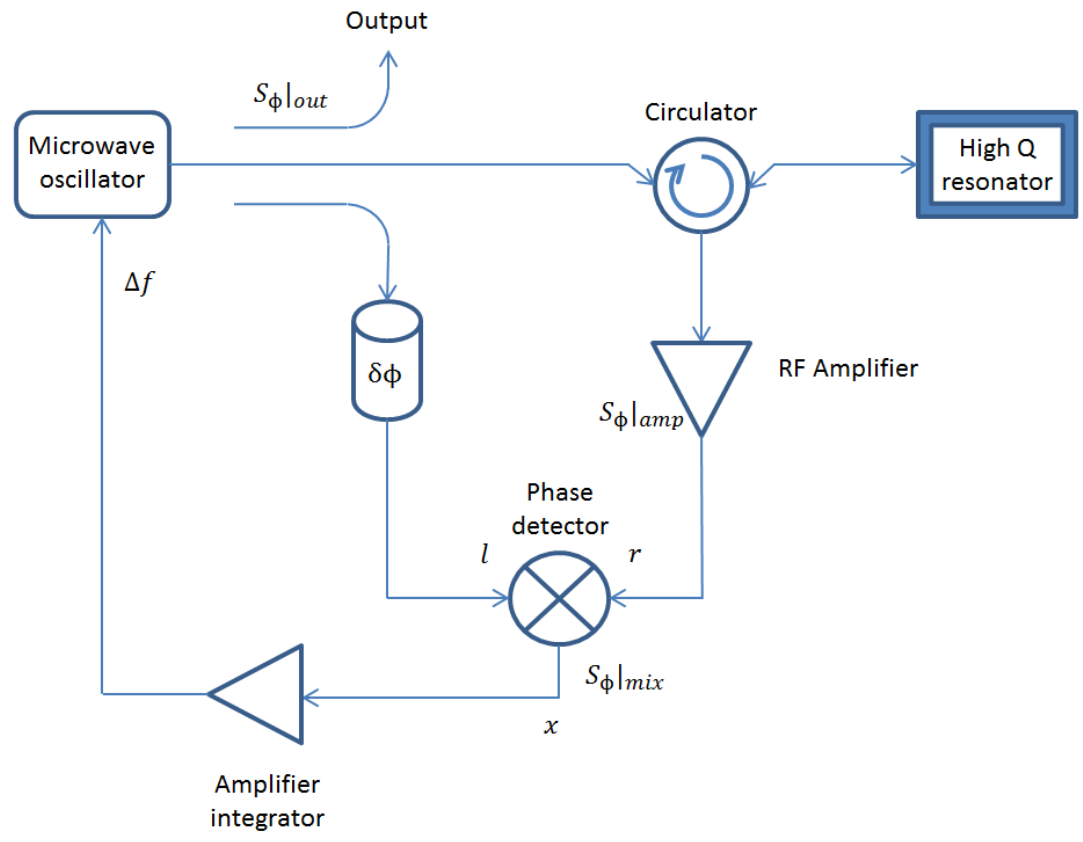

Figure 2.20: STALO configuration improved by suppressing the carrier and amplifying the signal before the mixer [18].

The carrier suppression is achieved by critically coupling to a one port resonator. If the signal to the resonator is on resonance, no signal is returned, thus the carrier is suppressed. As stated, the multiplicative phase noise of the amplifier is reduced when the carrier is suppressed. This can be explained with the help of Figure 2.21 and Figure 2.22.

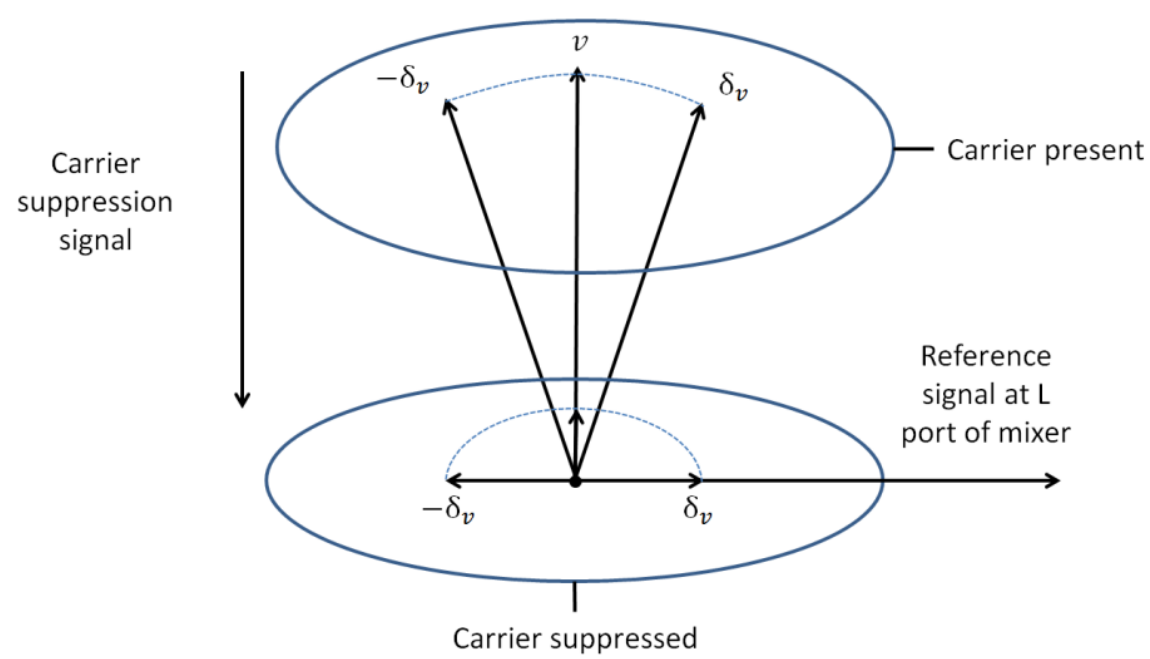

Figure 2.21: Phasor diagram showing a frequency error and the effect of suppressing the carrier before entering the RF amplifier [18]. 


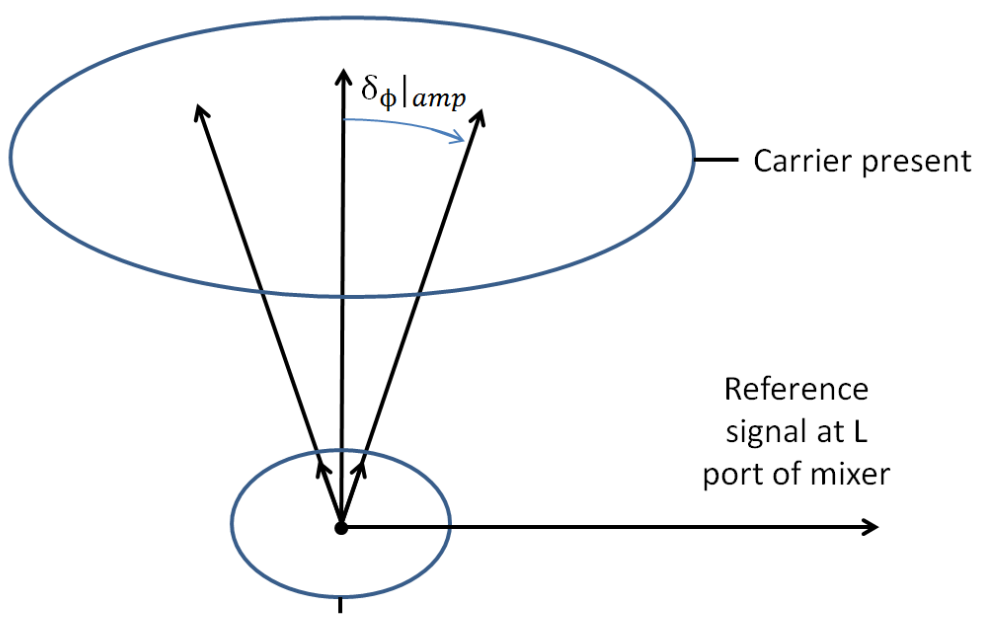

Carrier suppressed

Figure 2.22: Phasor diagram showing the phase noise contributed by the amplifier when the carrier is present and when the carrier is suppressed [18].

Figure 2.21 shows a phasor representation of the signals emanating from the resonator in the presence of frequency fluctuations in the oscillator. The case with the carrier present corresponds to the oscillator shown in Figure 2.19, while the case where the carrier is suppressed corresponds to the oscillator in Figure 2.20. The component of the resonator signal that is in-phase with the reference signal is unchanged by suppressing the carrier, indicating that no signal phase or signal-tonoise ratio information is lost by suppressing the carrier. The effect of the amplifier phase noise on the resonator signal with and without carrier suppression is shown in Figure 2.22. It indicates that the noise contributed by the amplifier is reduced when the carrier is suppressed. It follows that by suppressing the carrier and amplifying the resulting signal before entering the mixer can substantially improve the phase noise performance of a STALO.

In 2007, C.W. Nelson, D.A. Howe and A. Sen Gupta published a paper entitled, "Ultra-low-noise cavity-stabilized microwave reference oscillator using an air-dielectric resonator" [22], in which the techniques described above were used to design and measure an oscillator. In this design, a cylindrical waveguide cavity operating in the $\mathrm{TE}_{023}$ mode is used as the resonator to stabilize a Gunndiode oscillator, with the output taken through the almost critically coupled cavity. The schematic of this approach is shown in Figure 2.23. 


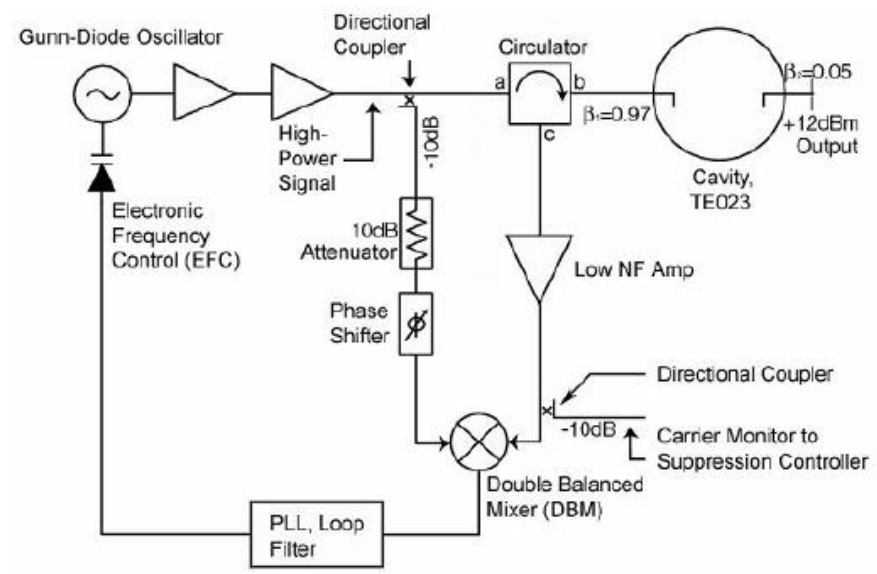

Figure 2.23: STALO configuration designed by Nelson, Howe and Gupta [22].

To further improve the phase noise performance, more techniques were incorporated in this configuration. These techniques, however, still mainly focus on supressing the carrier to reduce the noise contributed by the amplifier and minimizing the noise of the detector. The measured phase noise of this configuration is shown in Figure 2.24.

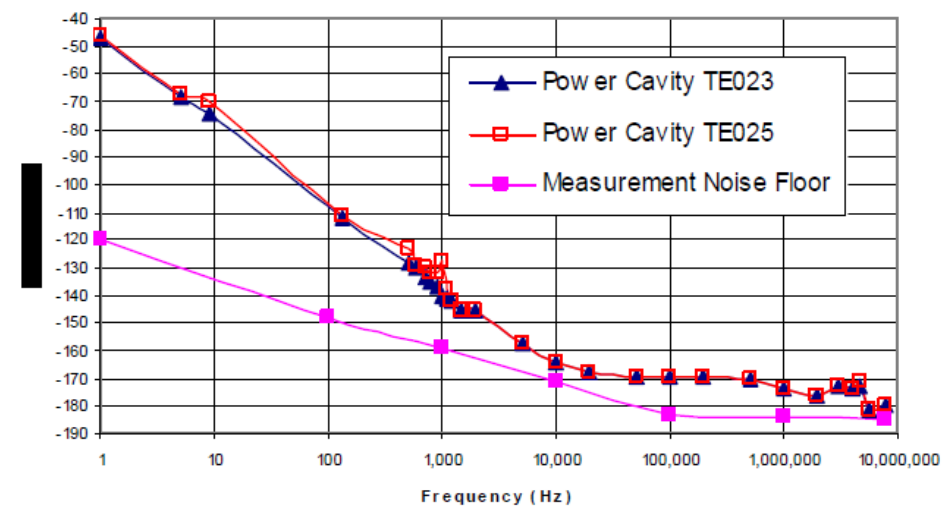

Figure 2.24: Measured phase noise of STALO in Figure 1-26 [22]. 


\section{Chapter 3}

\section{Design and realization}

In the following chapter, the frequency selective elements in the oscillator loop will be designed. The designs include a resonator (the frequency selective element) and a filter (used to suppress spurious modes of the resonator). Both elements are constructed from metallic waveguide cavities with air dielectrics. The designs consist of analytical calculations, simulations performed in CST and optimizations. The elements are designed to operate at a frequency of $9.2 \mathrm{GHz}$. The coupling to the resonator is designed at an optimum point to result in minimum phase noise, while the dimensions of the cavity are designed to result in the highest Q-factor.

\subsection{Resonator}

\subsubsection{Theory}

A resonator with a tuneable centre frequency and high Q-factor is described and designed in this section. Waveguide cavity resonators have very high power handling capability due to the metallic nature of the structure.

Transmission lines consisting of one or more conductors can support TE (transverse electric) and TM (transverse magnetic) modes of propagation. An unloaded or uniformly loaded waveguide can only support TE and TM modes. For a cylindrical waveguide, a mode's cutoff frequency, taking $z$ as the axial direction, is [23]

$$
\begin{aligned}
& \left(f_{c}\right)_{m n}^{T E^{Z}}=\frac{\chi_{m n}^{\prime}}{2 \pi a \sqrt{\mu \epsilon}} \\
& \left(f_{c}\right)_{m n}^{T M^{Z}}=\frac{\chi_{m n}}{2 \pi a \sqrt{\mu \epsilon}}
\end{aligned}
$$

and the resonant frequency of the cavity is given by

$$
\begin{aligned}
& \left(f_{r}\right)_{m n p}^{T E^{z}}=\frac{1}{2 \pi \sqrt{\mu \epsilon}} \sqrt{\left(\frac{\chi_{m n}^{\prime}}{a}\right)^{2}+\left(\frac{p \pi}{h}\right)^{2}} \\
& \left(f_{r}\right)_{m n p}^{T M^{z}}=\frac{1}{2 \pi \sqrt{\mu \epsilon}} \sqrt{\left(\frac{\chi_{m n}}{a}\right)^{2}+\left(\frac{p \pi}{h}\right)^{2}}
\end{aligned}
$$

where 
- $\mu=$ permeability of the medium

- $\epsilon=$ permittivity of the medium

- $\mathrm{m}=$ number of full-period variations of $E_{r}$ (TE modes) or $H_{r}$ (TM modes) with respect to $\theta(m=0,1,2, \ldots)$

- $\mathrm{n}=$ number of half-period variations of $E_{\theta}$ (TE modes) or $H_{\theta}$ (TM modes) with respect to $r(n=1,2,3, \ldots)$

- $\mathrm{p}=$ number of half-period variations of $E_{r}$ (TE modes) or $H_{r}$ (TM modes) with respect to $z$ ( $p=1,2,3, \ldots$ for TE and $p=0,1,2,3, \ldots$ for TM modes)

- $\mathrm{h}=$ height of cylindrical cavity

- $\chi_{m n}=$ nth zero $(n=1,2,3, \ldots)$ of the Bessel function $J_{m}$ of the first kind of order $(m$ $=0,1,2, \ldots)$

- $\chi_{m n}^{\prime}=$ nth zero $(n=1,2,3, \ldots)$ of the derivative of the Bessel function $J_{m}$ of the first order $m(m=0,1,2, \ldots)$

The field configurations of the first $15 \mathrm{TE}^{2}$ and $\mathrm{TM}^{2}$, obtained from [24], are illustrated in Figure 3.1. The solid and dashed lines represent the E-field and $\mathrm{H}$-field, respectively.
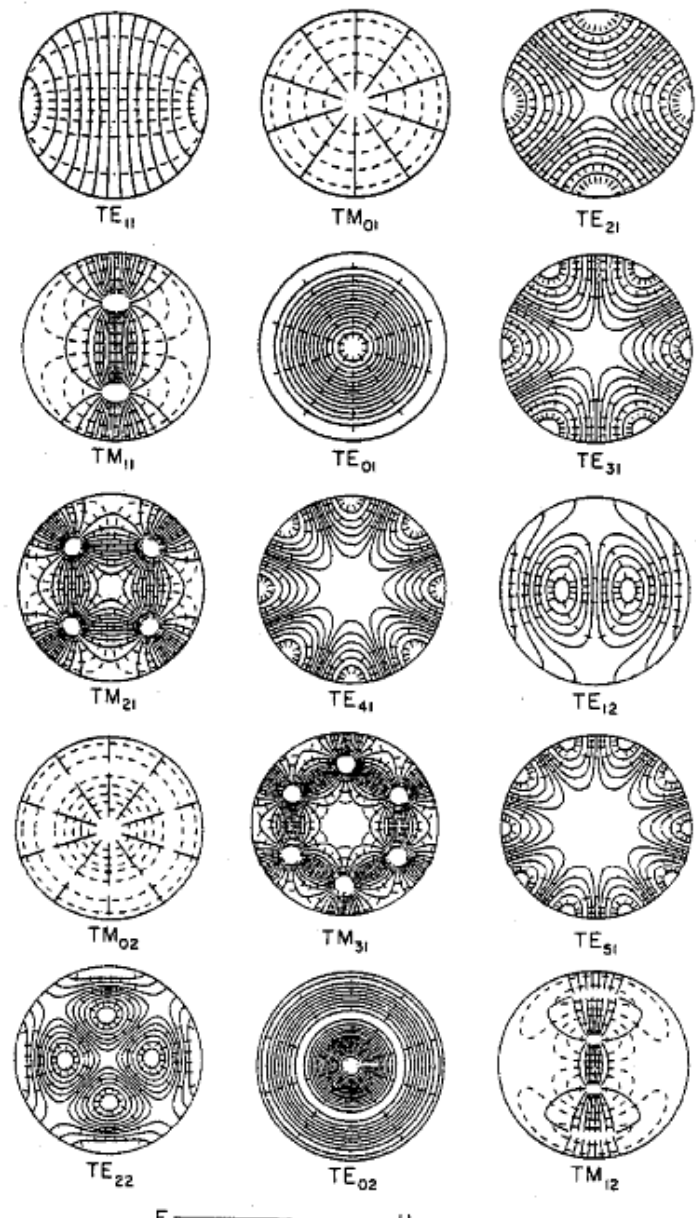

Figure 3.1: Field configurations of the first 15 modes for a cylindrical waveguide [24].

The $\mathrm{TE}_{01}$ mode is a popular mode due to the fact that it has a much higher Q-factor than the dominant $\mathrm{TE}_{11}$ mode and that it has no axial currents $\left(\mathrm{H}_{\theta}=0\right)$. Figure $3.2(\mathrm{a})$ and (b) show the surface 
currents, obtained from CST, flowing in a cylindrical cavity operating at an X-band frequency in the $\mathrm{TE}_{011}$ mode.

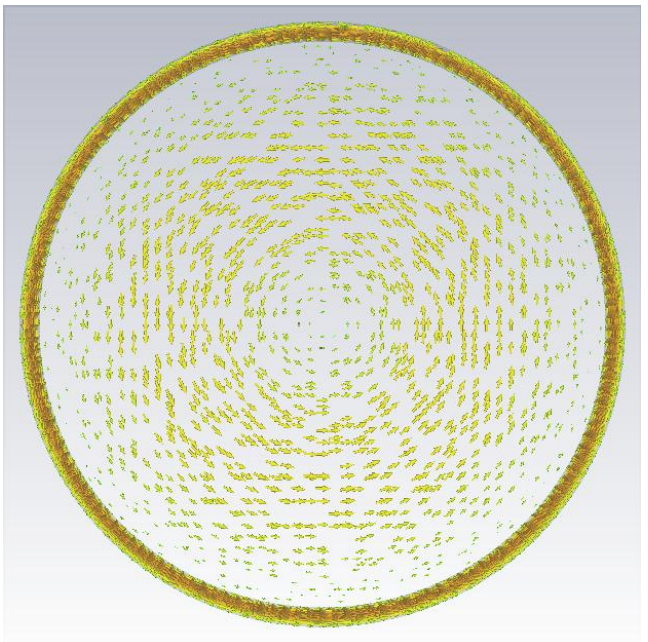

(a)

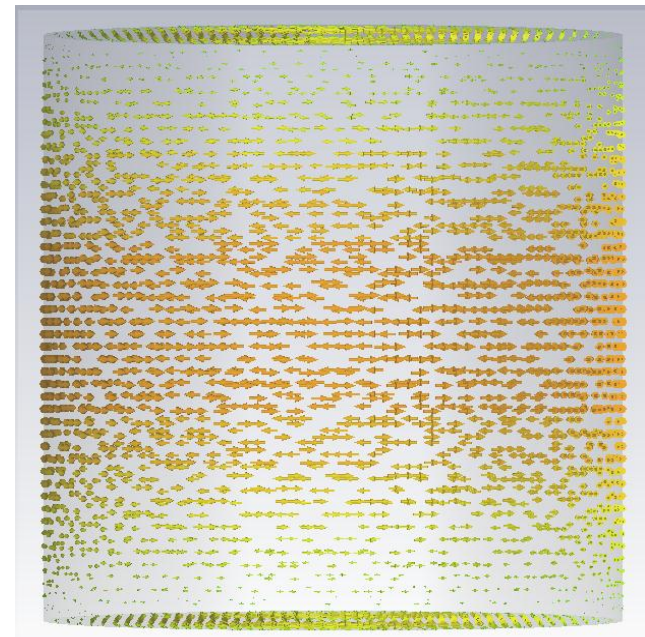

(b)

Figure 3.2: Surface currents obtained from CST simulation of cylindrical $T E_{011}$ mode (a) on top/bottom lid and (b) on cylindrical outer surface.

Should two modes have the same resonant frequency (but with different field patterns), they are referred to as degenerate. The $\mathrm{TE}_{01}$ and $\mathrm{TM}_{11}$ modes fall into this category.

Figure 3.3 to Figure 3.6 shows the peak electric and magnetic fields of the degenerate $\mathrm{TE}_{011}$ and $\mathrm{TM}_{111}$ modes, obtained from a CST simulation. 


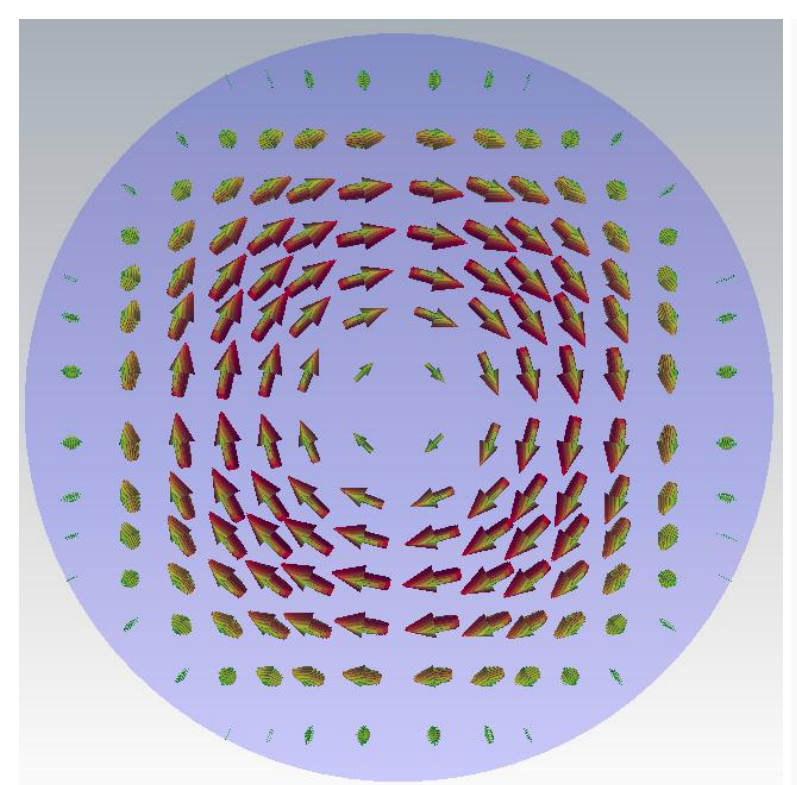

(a)

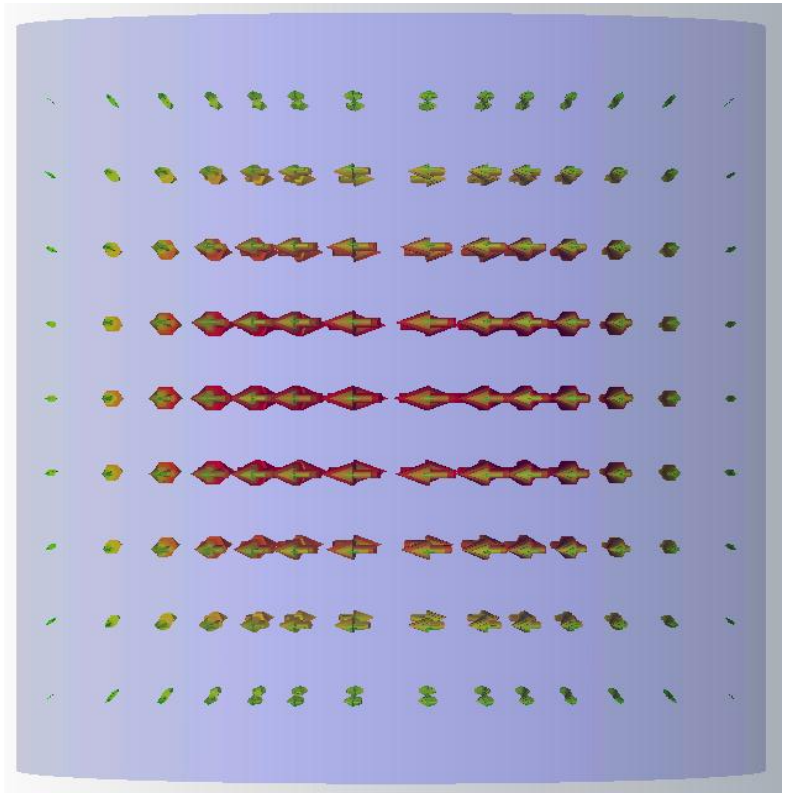

(b)

Figure 3.3: (a) Top view at $z=d$ and (b) side view of E-field patterns of the cylindrical TE $E_{011}$ mode, obtained from a CST simulation.

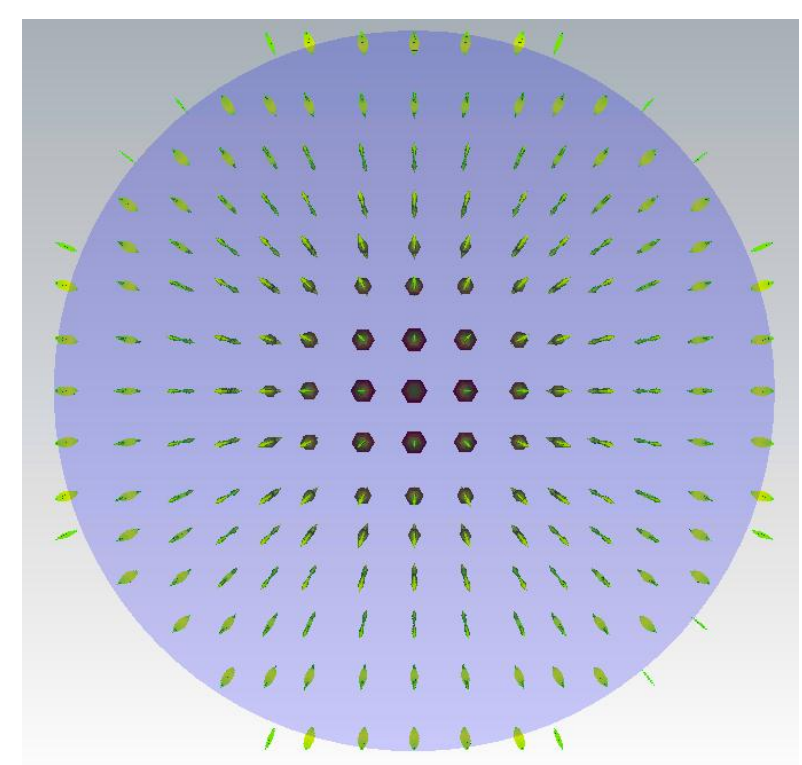

(a)

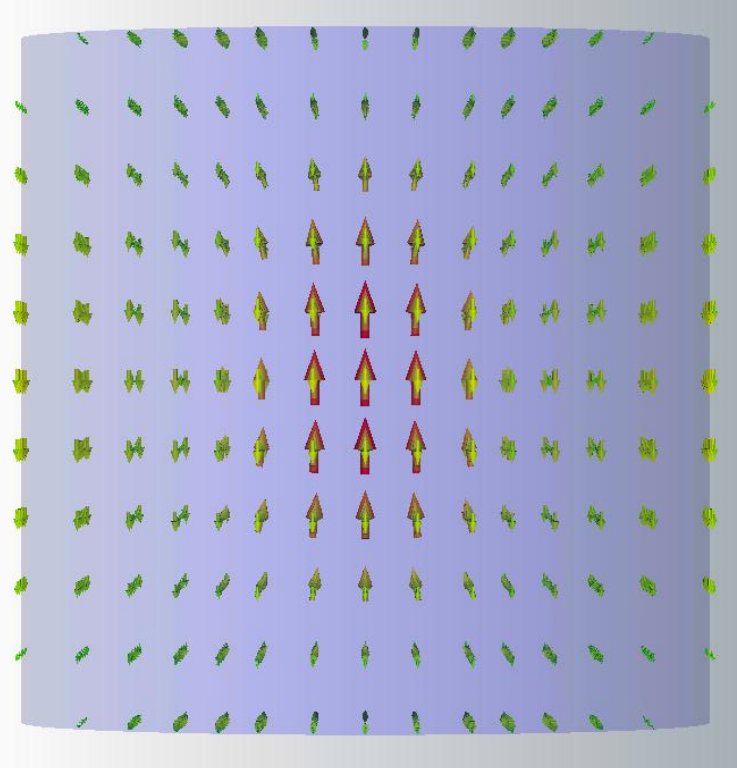

(b)

Figure 3.4: (a) Top view at $\mathrm{z}=d$ and (b) side view of H-field patterns of the cylindrical $T E_{011}$ mode, obtained from a CST simulation. 


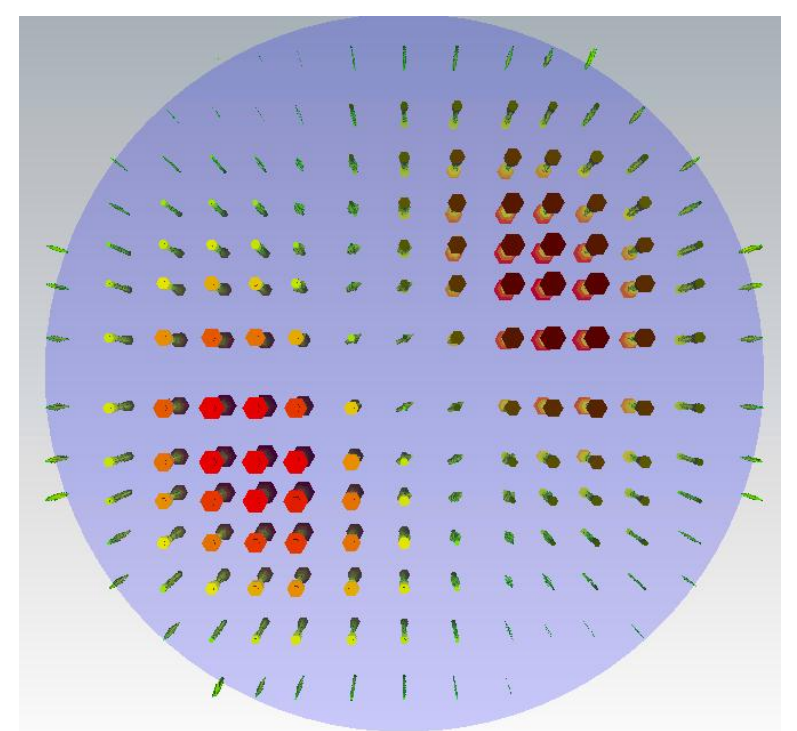

(a)

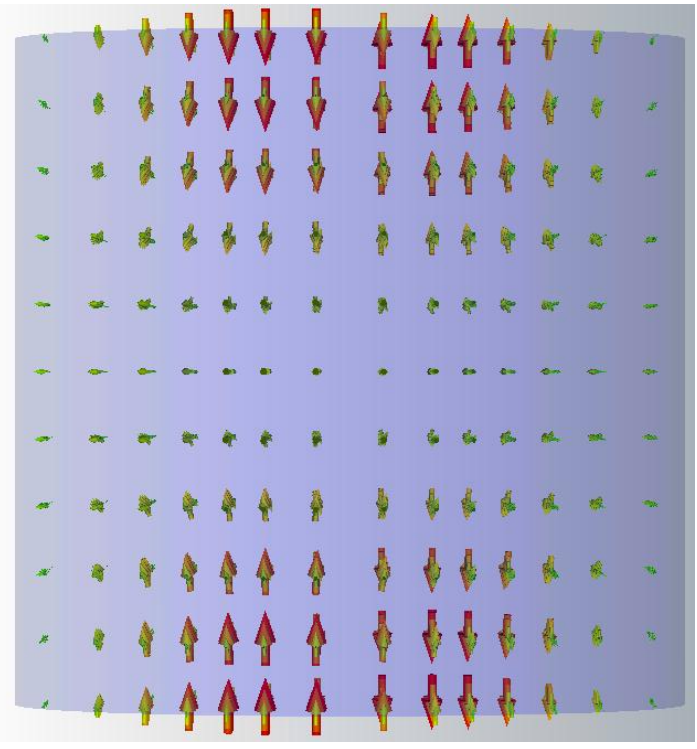

(b)

Figure 3.5: (a) Top view at $z=d$ and (b) side view of E-field patterns of the cylindrical TM ${ }_{111}$ mode, obtained from a CST simulation.

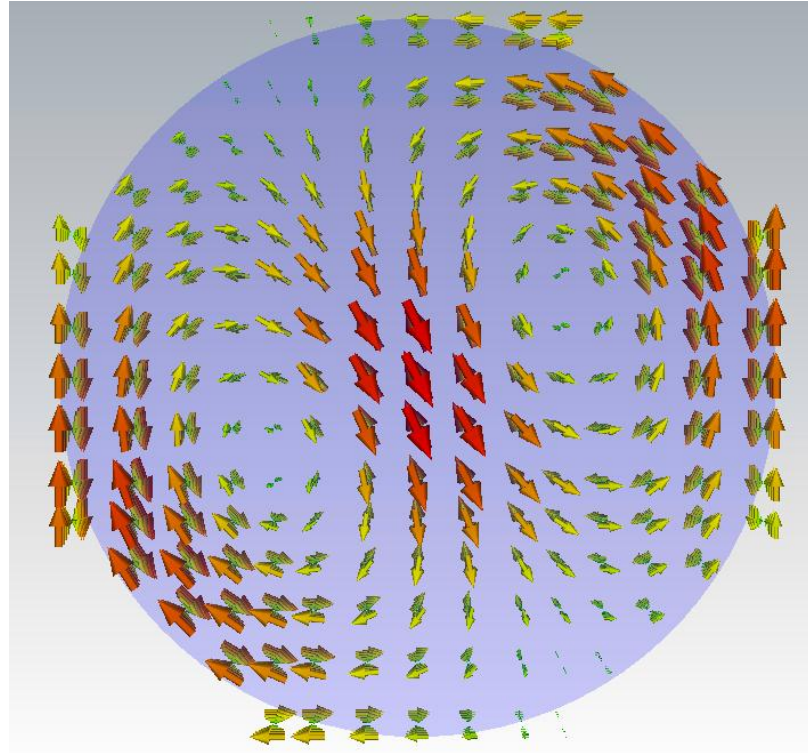

(a)

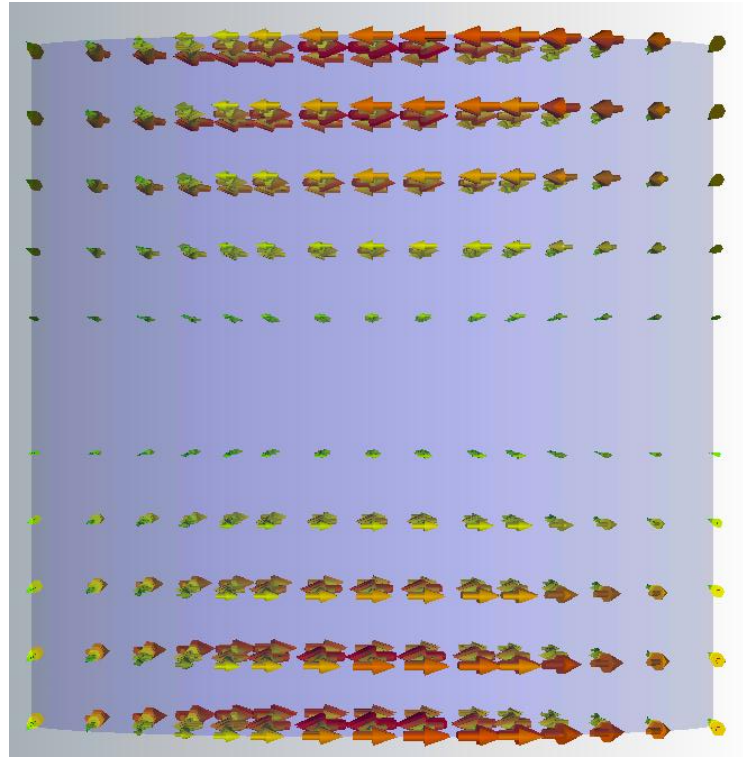

(b)

Figure 3.6: (a) Top view at $\mathrm{z}=d$ and (b) side view of $\mathrm{H}$-field patterns of the cylindrical $T M_{111}$ mode, obtained from a CST simulation.

\subsubsection{Design}

For a given mode, there exists an optimum ratio between the length and radius of the cavity in order to obtain a maximum $Q$-factor. Figure 3.7 shows a $Q$ chart with a plot of the dominant $\mathrm{TE}_{111}$ mode and the $\mathrm{TE}_{011}$ mode, where deltaS is the skin depth and Lambda0 is the free-space wavelength [25]. The general equation to construct a $Q$ chart for the TE modes can be obtained from [25]. 


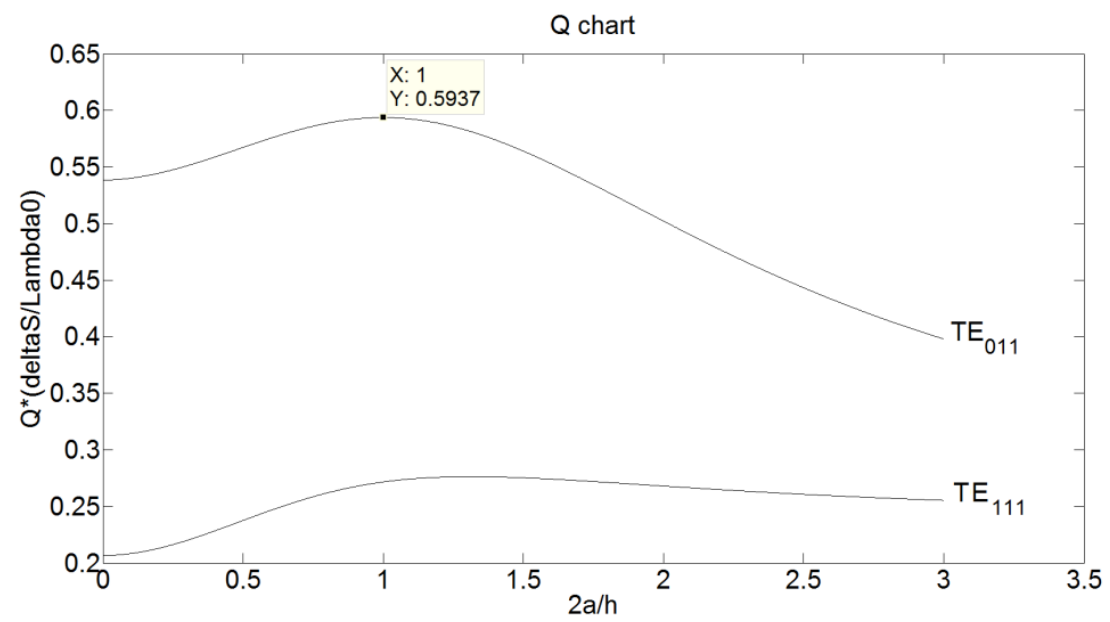

Figure 3.7: $Q$ chart of cylindrical waveguide cavity, showing the $T E_{011}$ mode and the dominant $T E_{111}$ mode.

It can be seen that the $\mathrm{TE}_{011}$ mode has a higher $\mathrm{Q}$ than that of the dominant $\mathrm{TE}_{111}$ mode for any dimension ratio. The maximum $Q$ point for the $\mathrm{TE}_{011}$ mode occurs at a cavity ratio of

$$
\frac{2 a}{h}=1
$$

Inserting (3.5) in (3.3) and rearranging, results in

$$
a=\sqrt{\frac{4\left(\chi_{n m}^{\prime}\right)^{2}+(l \pi)^{2}}{16\left(f_{\text {res }}\right)^{2} \pi^{2} \mu \epsilon}}
$$

With

$$
\begin{array}{ll}
\chi_{n m}^{\prime} & =3.8318 \\
l & =1 \\
f_{\text {res }} & =9.2 \mathrm{e} 9 \mathrm{~Hz} \\
\mu & =4 \pi \times 10^{-7} \mathrm{H} / \mathrm{m} \\
\epsilon & =8.854 \times 10^{-12} \mathrm{~F} / \mathrm{m}
\end{array}
$$

results that

$$
\begin{aligned}
\text { a } & =21.4788 \mathrm{~mm} \\
\text { h } & =42.9576 \mathrm{~mm}
\end{aligned}
$$

These dimensions will result in a maximum unloaded Q-factor for a cylindrical waveguide cavity resonator operating at $9.2 \mathrm{GHz}$ in the $\mathrm{TE}_{011}$ mode. Using these dimensions, Figure 3.8 and Figure 3.9 shows maps of the cutoff- and resonant frequencies of the modes within the frequency band of 4 $\mathrm{GHz}$ to $12.4 \mathrm{GHz}$, respectively. 
Cutoff frequencies of different modes

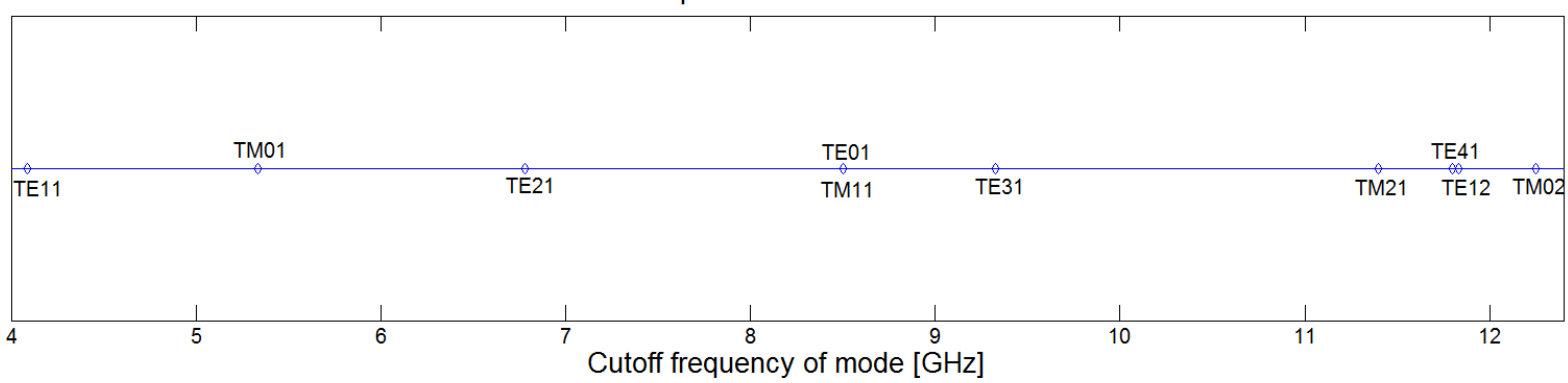

Figure 3.8: Cutoff frequencies of different modes of cylindrical waveguide with dimensions of $a=21.48 \mathrm{~mm}$.

Resonant frequencies of different modes

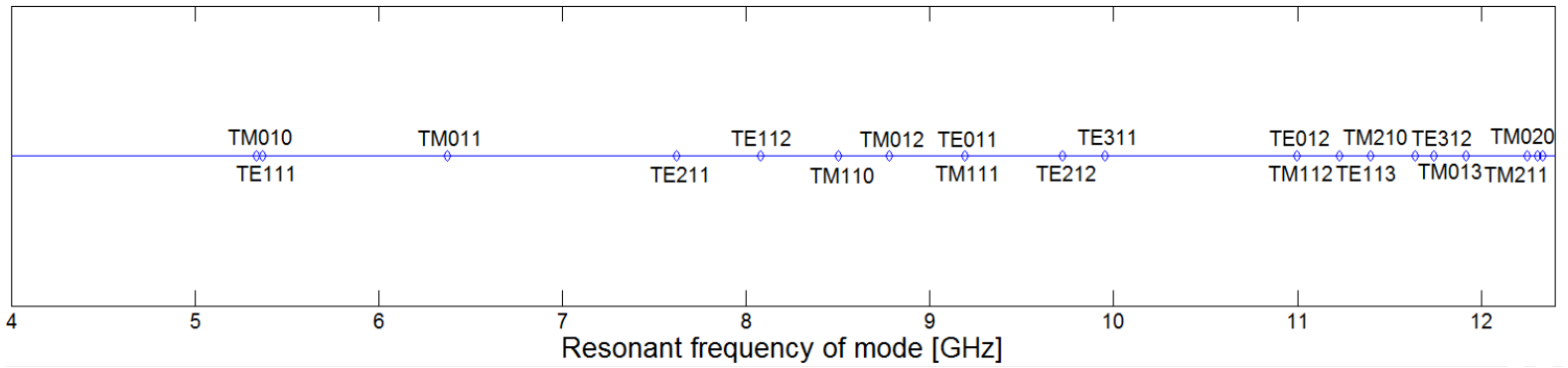

Figure 3.9: Resonant frequencies of different modes of cylindrical waveguide cavity with dimensions of $a=$

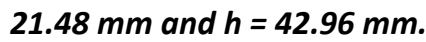

\subsubsection{Separating degenerate modes and tuning centre frequency of $\mathrm{TE}_{011}$ mode}

The $\mathrm{TM}_{111}$ and $\mathrm{TE}_{011}$ modes are degenerate. Special care must thus be taken to ensure that one does not couple to the wrong mode, or both modes.

According to [26], "if a small perturbation is made in one of the cavity walls, it will in general change one type of energy more than the other and the resonant frequency would then shift by an amount necessary to again equalize the energies".

Equation (3.7) , obtained from [26], indicates that if the volume that is removed from the cavity by inserting the screw predominately consists of magnetic energy, the resonant frequency will increase. Also, if the volume removed from the cavity by inserting the screw mainly consists of electric energy, the resonant frequency will decrease.

$$
\begin{aligned}
\frac{\Delta \omega_{0}}{\omega_{0}} & =\frac{\int_{\Delta v}\left(\mu H^{2}-\epsilon E^{2}\right) d v}{\int_{v}\left(\mu H^{2}+\epsilon E^{2}\right) d v} \\
& =\frac{\Delta U_{H}-\Delta U_{E}}{U}
\end{aligned}
$$

No surface currents flow in the axial direction of the $\mathrm{TE}_{01}$ mode. A tuning screw can be inserted at the top or bottom endplate of the cavity without introducing any significant power loss. It follows that the $\mathrm{Q}$-factor of the $\mathrm{TE}_{01}$ mode will not be affected, due to the fact that no currents will flow in 
the axial direction on the screw and through the contact with the endplate. Figure 3.10 (a) and (b) illustrate this.

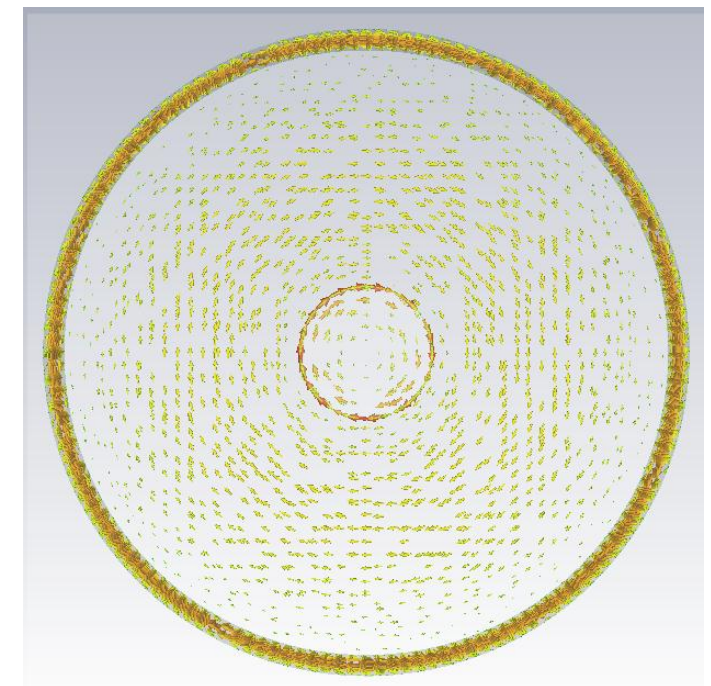

(a)

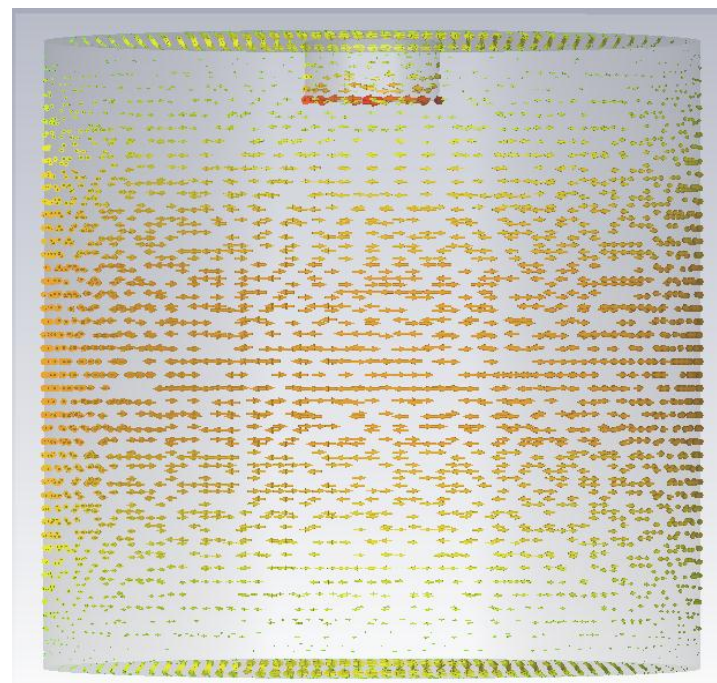

(b)

Figure 3.10: (a) Top and (b) side view of the surface currents flowing in the $T E_{011}$ mode cavity, together with the surface currents flowing on the tuning screw.

The tuning screw is inserted in the middle of one of the endplates in the axial direction, at the maximum $\mathrm{H}$-field, in order to obtain a maximum frequency shift. A screw with a large diameter was chosen in order to maximise the tuning effect. The screw chosen for the simulations and which will be used in the actual structure is the Temex AT 6965-0 tuning screw, which has a diameter of 8.8 $\mathrm{mm}$ and a maximum length of $12.7 \mathrm{~mm}$. The datasheet is available online at http://www.temex-ceramics.com/site/fichiers/DatasheetTuning.pdf. Figure 3.11 and Figure 3.12 shows the resonant frequencies of the $\mathrm{TM}_{111}$ and $\mathrm{TE}_{011}$ modes, respectively, obtained from a parameter sweep from CST with the length of the tuning screw varied.

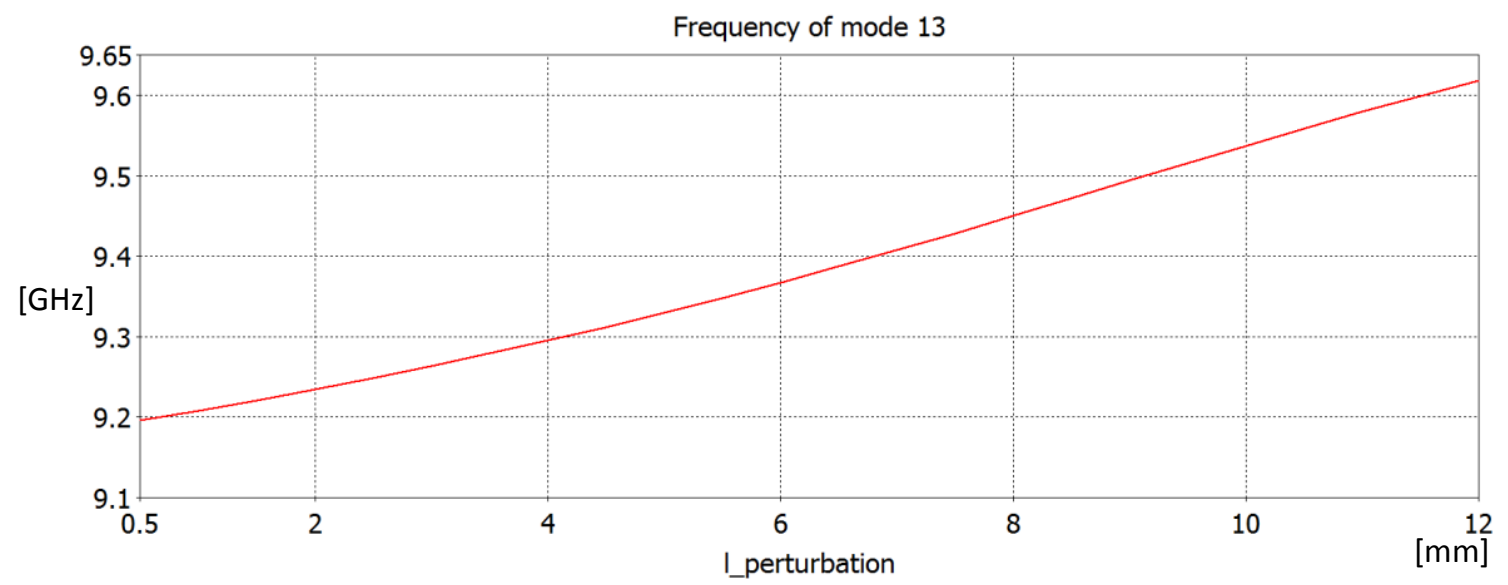

Figure 3.11: Resonant frequency of the $T M_{111}$ mode by varying the length of the tuning screw. 


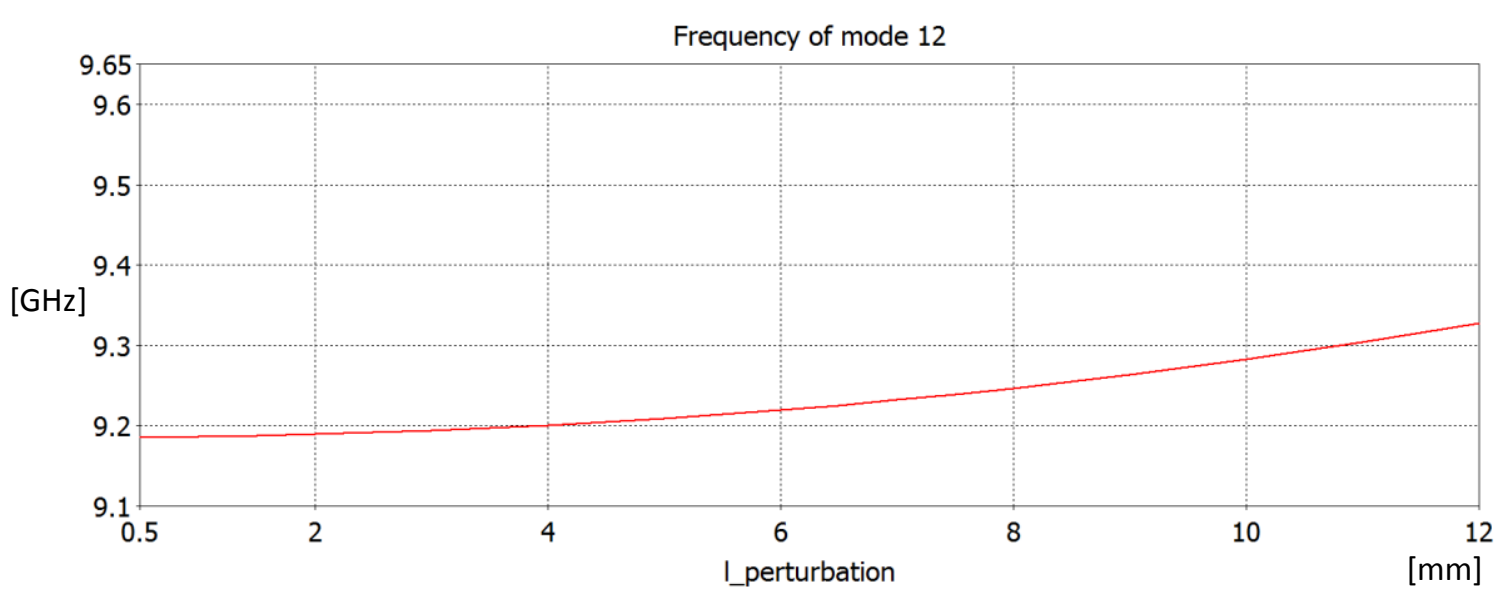

Figure 3.12: Resonant frequency of the $T E_{011}$ mode by varying the length of the tuning screw.

It can be seen that the resonant frequency of the $\mathrm{TM}_{111}$ mode is affected more than that of the $\mathrm{TE}_{011}$ mode. The fact that the maximum $\mathrm{H}$-field of the $\mathrm{TM}_{111}$ mode occurs closer to the endplates than that of the $\mathrm{TE}_{011}$ mode can explain this phenomenon. The nominal length of the screw is chosen as $4 \mathrm{~mm}$, where it can be seen from Figure 3.11 and Figure 3.12 that the resonant frequency of the $\mathrm{TM}_{111}$ mode is approximately $100 \mathrm{MHz}$ from the resonant frequency of the $\mathrm{TE}_{011}$ mode, which has a centre frequency of $9.2 \mathrm{GHz}$ with this length.

The above experiment shows that by inserting a perturbation screw in the middle of one of the endplates separates the degenerate $\mathrm{TM}_{111}$ and the $\mathrm{TE}_{011}$ modes while also serving as a tuning mechanism for the wanted $\mathrm{TE}_{011}$ mode.

Figure 3.13 shows a map of the resonant frequencies of other spurious modes with the perturbation screw at its nominal length. The individual resonant frequencies of these modes were obtained through an eigenmode simulation in CST and plotted with MATLAB.

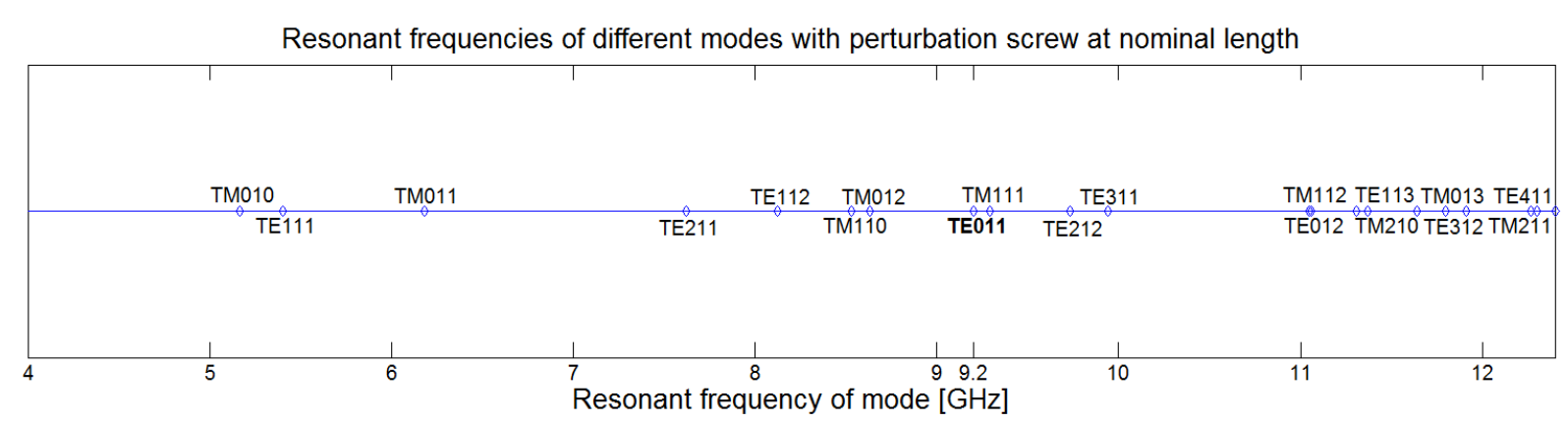

Figure 3.13: Resonant frequencies of different modes with the tuning screw inserted into the cavity at the nominal length (4 $\mathrm{mm})$.

\subsubsection{Coupling and excitation}

In order for the resonator to minimise the phase noise of the oscillator, a quarter of the power available from the source must be dissipated in the load, a quarter in the source and a half in the resonator [16]. This statement will be derived again below.

The output PSD of the oscillator's phase noise (2.34) is shown here again as 


$$
S_{\phi}(f)=\left[1+\frac{1}{f^{2}}\left(\frac{v_{0}}{2 Q_{L}}\right)^{2}\right] S_{\psi}(f)
$$

When the offset frequency is smaller than the half bandwidth of the resonator $\left(f<\frac{v_{0}}{2 Q_{L}}\right)$, the output PSD of the phase noise becomes

$$
S_{\phi}(f)=\left(\frac{v_{0}}{2 Q_{l} f}\right)^{2} S_{\psi}(f)
$$

If the additive noise of the input PSD, $\mathrm{S}_{\psi}(\mathrm{f})$, is white noise, the noise is flat with frequency. Looking at only the additive noise component, according to [15], the two-sided PSD of a feedback oscillator with an effective noise figure of $F$ is

$$
S_{\psi}(f)=\frac{2 F K T}{P_{\text {in }}}
$$

Combining (3.9) and (3.10), results in the output PSD of the phase noise as

$$
S_{\phi}(f)=\left(\frac{v_{0}}{2 Q_{l} f}\right)^{2} \frac{2 F K T}{P_{\text {in }}}
$$

By inspecting (3.11), it can be seen that by increasing the input power to the active device and the loaded Q-factor of the resonator, the output PSD of the oscillator's phase noise will decrease. The output PSD of the phase noise is thus inversely proportional to the product of the square of the loaded Q-factor and the input power to the active device. It can be stated mathematically as

$$
S_{\phi}(f) \propto \frac{1}{P_{\text {in }} Q_{l}^{2}}
$$

It is important to note that the loaded Q-factor cannot be increased indefinitely as the insertion loss increases proportional to $Q_{l}$ and this in turn increases the phase noise [4]. There exists an optimum point where it will result in minimum phase noise. This condition is satisfied if the input and output couplings are equal [4]. This will also ensure maximum power transfer from port 1 to port 2, whilst minimising the phase noise. It is now necessary to find these coupling values, which will be derived below.

Figure 3.14 shows a lumped element model of a microwave resonator near resonance, coupled to a load and source. The transformers represent the coupling between the source and the resonator and resonator and the load. 

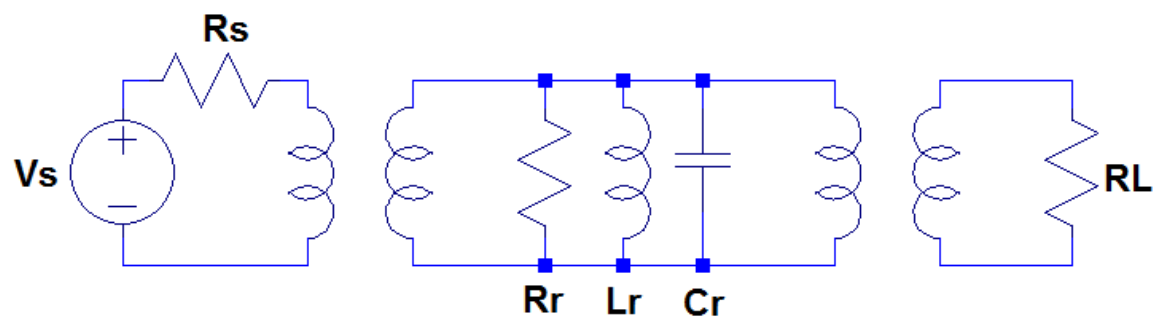

Figure 3.14: Lumped element model of microwave resonator near resonator, connected to a source and a load, where the transformers represent the coupling.

When the source and load resistances are transformed to the resonator and the circuit is at resonance, the model becomes as illustrated in Figure 3.15.

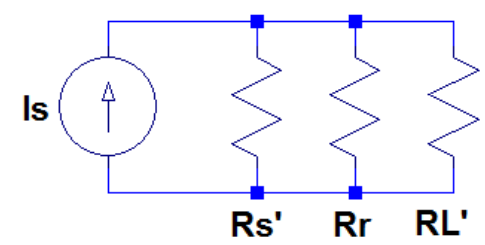

Figure 3.15: Equivalent model of Figure 3.14 when the circuit is at resonance and the load and source resistances are transformed to the resonator.

where

- $\mathrm{V}_{\mathrm{S}} \quad$ = Source voltage [V]

- $\mathrm{L}_{\mathrm{r}} \quad=$ Model of effective resonator inductance $[\mathrm{H}]$

- $\mathrm{C}_{\mathrm{r}} \quad=$ Model of effective resonator capacitance $[\mathrm{F}]$

- $\mathrm{R}_{\mathrm{r}} \quad=$ Resistance of the resonator $[\Omega]$

- $\mathrm{R}_{\mathrm{s}} \quad=$ Source resistance $[\Omega]$

- $\mathrm{R}_{\mathrm{L}} \quad=$ Load resistance $[\Omega]$

- $\mathrm{I}_{\mathrm{s}} \quad=$ Current source $[\mathrm{A}]$

The parallel combination of the resistors in Figure 3.15 can be written as

$$
G_{t o t}=G_{s}+G_{r}+G_{L}
$$

where the $\mathrm{G}=\frac{1}{R}$ is the conductance of the individual resistances of Figure 3.15, in Siemens [S]. Referring to Figure 3.14 and Figure 3.15 , the unloaded and loaded Q-factor can be written as

$$
Q_{o}=\frac{1}{\omega_{0} L_{r} G_{r}}
$$




$$
\begin{aligned}
Q_{l} & =\frac{1}{\omega_{0} L_{r} G_{t o t}} \\
& =\frac{G_{r}}{G_{t o t}} Q_{u}
\end{aligned}
$$

respectively. The voltage across each element is

$$
V=\frac{I_{s}}{G_{t o t}}
$$

The resonator's output power can be written as

$$
\begin{aligned}
P_{\text {out }} & =V^{2} G_{L} \\
& =\frac{I_{s}^{2}}{G_{\text {tot }}^{2}} G_{L}
\end{aligned}
$$

The power available from the source is

$$
P_{\text {available }}=\frac{\left|I_{S}\right|^{2}}{4 G_{S}}
$$

Writing (3.18) in terms of the currents source results that

$$
\left|I_{S}\right|^{2}=4 G_{s} P_{\text {available }}
$$

The power at the input of the active device can be represented by the power at the output of the resonator. Substituting (3.15) and (3.17) into (3.12), the output phase noise proportionality becomes

$$
S_{\phi}(f) \propto \frac{G_{t o t}^{4}}{I_{s}^{2} G_{L} G_{r}^{2} Q_{u}^{2}}
$$

Substituting (3.19) into (3.20), the proportionality becomes

$$
S_{\phi}(f) \propto \frac{G_{\text {tot }}^{4}}{4 G_{s} P_{\text {available }} G_{L} G_{r}^{2} Q_{u}^{2}}
$$

It is desirable for the input and output to be matched to the same characteristic impedance. Since the coupling of the source to resonator and resonator to the load are represented by the transformers, the information of the coupling is contained in the transformed resistances. Recall that the input and output couplings must be equal. This is equivalent to setting $G_{l}=G_{s}$. The power available from the source and the unloaded Q-factor can be treated as constants. Also, setting the 
resonator's conductance equal to a constant, the ratio of the source to resonator conductance can be found. (3.21) can now be written as

$$
S_{\phi}(f) \propto \frac{\left(2 G_{s}+1\right)^{4}}{G_{s}^{2}}
$$

where the resonator's conductance, the unloaded Q-factor and the power available from the source have been set to 1 . The point where the derivative of the phase noise with respect to the source conductance is equal to zero will indicate a maximum or minimum. Figure 3.16 shows a plot of (3.22).

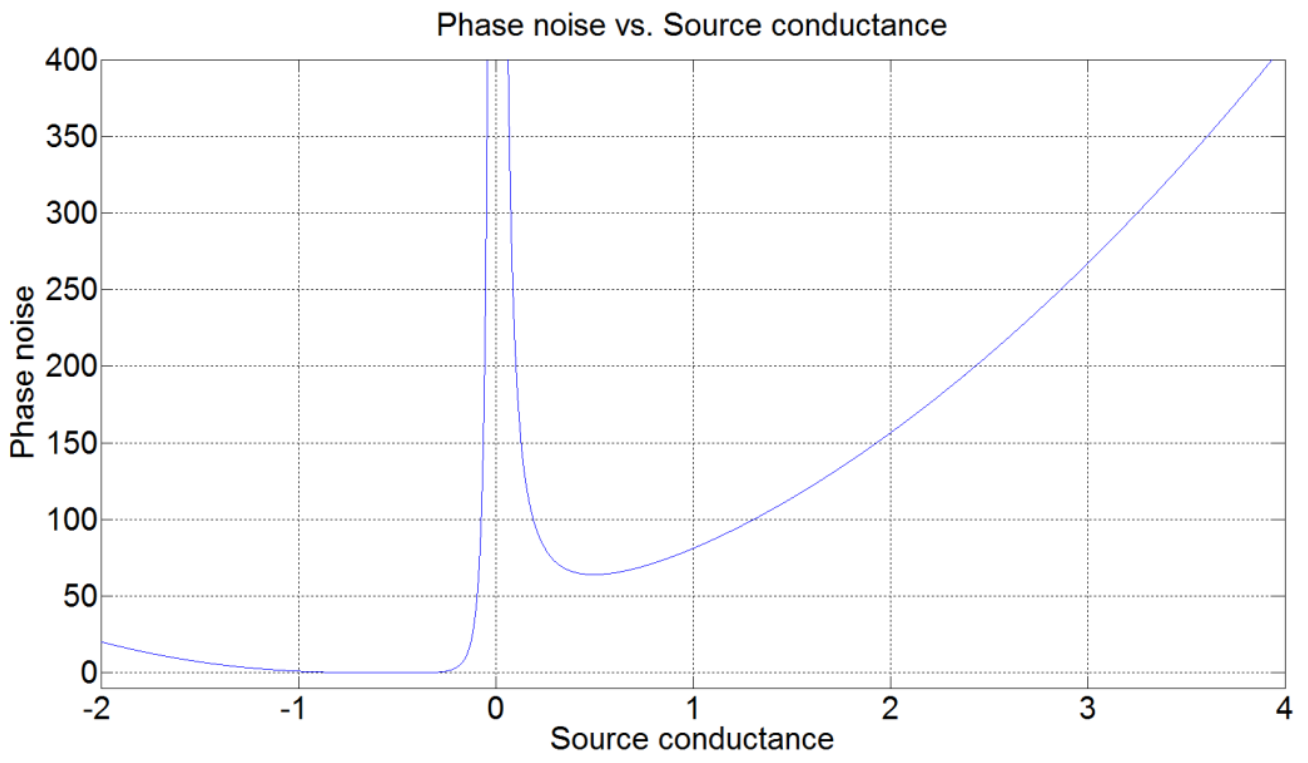

Figure 3.16: Plot of (3.22) illustrating that the phase noise with respect to the source conductance reaches a minimum when the derivative is zero.

It can be seen that, in the case where the conductance is positive, the phase noise with respect to the source conductance exhibits a minimum at its turning point. By taking the derivative of (3.22), setting it equal to zero and taking the positive root, will result in the ratio of resonator to source conductance to result in minimum phase noise. The derivative of the phase noise with respect to the source conductance is given as

$$
\frac{d S_{\phi}}{d G_{s}}=\frac{2\left(2 G_{s}-1\right)\left(2 G_{s}+1\right)^{3}}{G_{s}^{3}}
$$

Setting this equation equal to zero results in

$$
\frac{d S_{\phi}}{d G_{s}}=\frac{2\left(2 G_{s}-1\right)\left(2 G_{s}+1\right)^{3}}{G_{s}^{3}}=0
$$

or 


$$
2\left(2 G_{s}-1\right)\left(2 G_{s}+1\right)^{3}=0
$$

The roots of the equation are

$$
\begin{gathered}
G_{S}=0.5 \\
\text { or } \\
G_{S}=-0.5
\end{gathered}
$$

The positive root of the equation is $G_{s}=0.5$. Recall that the resonator's conductance was set to 1 . This indicates the following relationships between the conductance of the source, load and resonator as

$$
G_{s}=\frac{1}{2} G_{r}
$$

and

$$
G_{l}=G_{s}
$$

Inserting (3.28) and (3.29) into (3.15) results that

$$
Q_{l}=\frac{Q_{o}}{2}
$$

This states that in order for the resonator to minimise the phase noise of the output of the oscillator, the loaded Q-factor of the resonator must be exactly half of the unloaded Q-factor. For design and measurement purposes, it is necessary to state this in terms of S-parameters and is derived below.

Referring to Figure 3.15 and the results from (3.28) and (3.29), if we then set

$$
\begin{aligned}
& I_{S}=2 \mathrm{~A} \\
& G_{S}=1 \mathrm{~S} \\
& G_{L}=1 \mathrm{~S} \\
& G_{r}=2 \mathrm{~S}
\end{aligned}
$$

the power available from the source is

$$
\begin{aligned}
P_{\text {available }} & =\frac{\left|I_{S}\right|^{2}}{4 G_{S}} \\
& =1 \mathrm{~W}
\end{aligned}
$$

With $Z_{\text {in }}$ equal to the resonator resistance in parallel with the load resistance, 


$$
\begin{aligned}
Z_{\text {in }} & =\frac{(1)(0.5)}{1+0.5} \\
& =1 / 3
\end{aligned}
$$

and with the characteristic impedance of port 1 (the source impedance),

$$
Z_{0}=1,
$$

the reflected wave looking into port 1 is

$$
\begin{aligned}
S_{11} & =\left.\frac{V_{1}^{+}}{V_{1}^{-}}\right|_{V_{2}^{+}=0} \\
& =\left.\Gamma\right|_{V_{2}^{+}=0} \\
& =\frac{Z_{\text {in }}-Z_{0}}{Z_{\text {in }}+Z_{0}} \\
& =-0.5 \\
\left|S_{11}\right|^{2} & =0.25
\end{aligned}
$$

Writing this in decibels results in

$$
10 \log \left|S_{11}\right|^{2}=\left|S_{11}\right|_{d B}=-6 d B
$$

The voltage across each element is

$$
\begin{aligned}
V & =\frac{2 \mathrm{~A}}{4 S} \\
& =0.5 \mathrm{~V}
\end{aligned}
$$

The power dissipated by the each element is

$$
\begin{gathered}
P=\frac{|V|^{2}}{R} \\
P_{S}=0.25 \mathrm{~W} \\
P_{L}=0.25 \mathrm{~W} \\
P_{r}=0.5 \mathrm{~W}
\end{gathered}
$$

The definition of $\left|S_{21}\right|^{2}$ is [27]

$$
\left|S_{21}\right|^{2}=\frac{\text { Power delivered to load }}{\text { Power available from source }}
$$


The power delivered to the load is $0.25 \mathrm{~W}$ and the power available from the source is $1 \mathrm{~W}$, indicating

$$
\left|S_{21}\right|_{d B}=-6 d B
$$

Due to the symmetry of the circuit, $\left|S_{22}\right|^{2}=\left|S_{11}\right|^{2}$ and $\left|S_{12}\right|^{2}=\left|S_{21}\right|^{2}$. Thus in order for the resonator minimise phase noise of the oscillator, the loaded $\mathrm{Q}$-factor must be equal to half the unloaded Q-factor. When this is realized, it will cause both the return loss and the insertion loss to be equal to $6 \mathrm{~dB}$.

\subsubsection{Simulations}

The unloaded Q-factor is found by simulating the resonator with the eigenmode solver in CST and then adding the effect of lossy materials in a post processing step. The material of the cavity is simulated as aluminium and that of the tuning screw as copper. Figure 3.17 shows the result of the unloaded Q-factor obtained from CST.

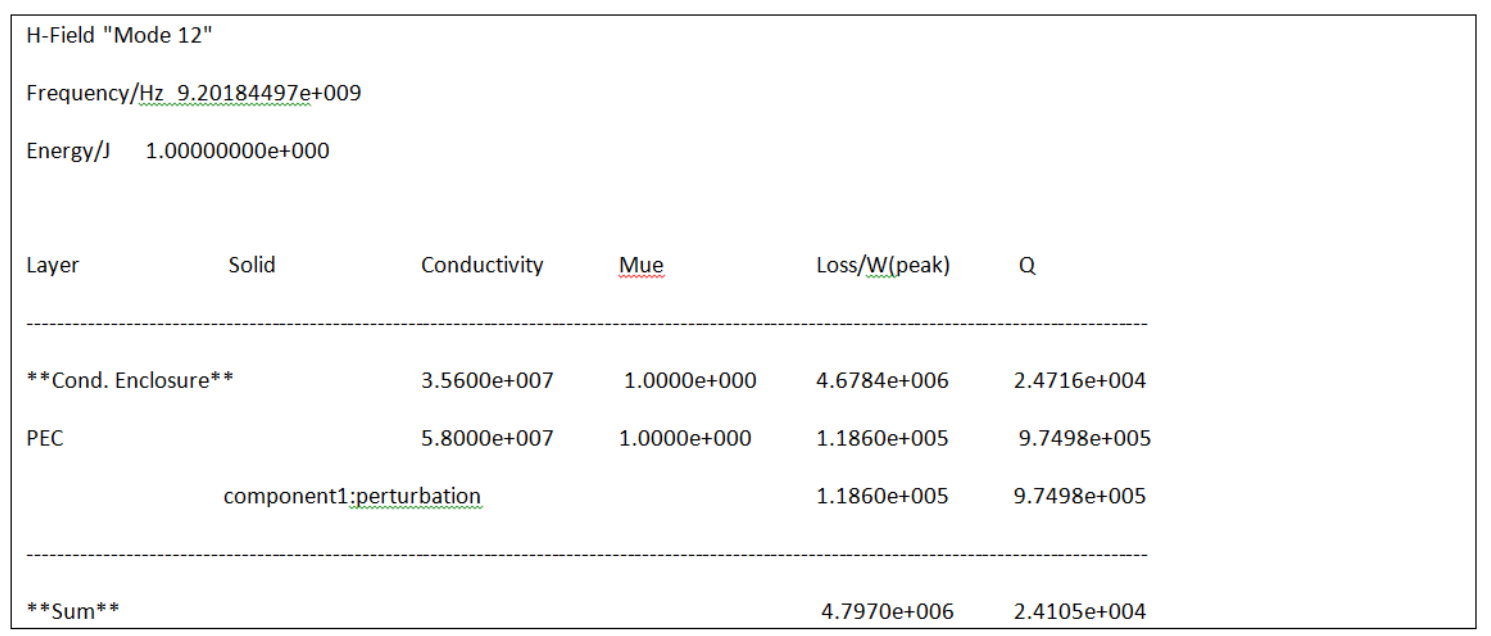

Figure 3.17: Unloaded Q-factor of resonator, obtained from a CST simulation.

Figure 3.17 indicates that the unloaded Q-factor of the resonator operating in the $\mathrm{TE}_{011}$ mode is

$$
Q_{o}=24105
$$

\subsubsection{Coupling}

In order to couple electromagnetic energy in and out of the resonator, some coupling scheme must be used. A common way that energy can be coupled in and out of a waveguide cavity resonator is by means of an aperture or a probe.

Bethe [28] developed a theory which states that when a small circular aperture is made in a common conducting plane of zero thickness between two waveguides and there exists a field on one side, then the field in the second region will be the same as that from an electric dipole $P_{0}$ normal to the wall and a magnetic dipole $M_{0}$ tangential to the wall at the centre of the aperture with the aperture closed [29]. These electric and magnetic dipoles are given by 


$$
\begin{gathered}
P_{0}=-\epsilon_{0} p_{e} E_{n} \\
M_{0}=-p_{m} H_{t}
\end{gathered}
$$

where $p_{e}$ and $p_{m}$ are the electric and magnetic polarizabilities of the aperture and $E_{n}$ and $H_{t}$ are the normal electric and tangential magnetic fields, respectively [29]. Thus when a normal electric field or tangential magnetic field is incident on an aperture in one waveguide, an electric or magnetic dipole will be set up in the aperture and radiate power into a given mode in the next waveguide, only when the magnetic/electric field of the mode to be excited has a component parallel to the dipole moment $p_{m} / p_{e}$, respectively [30]. This theory can be expanded to include electrically large apertures of arbitrary shape with a plane of finite thickness [30].

Figure 3.6 (a) and (b) shows the field configuration of the $\mathrm{TM}_{111}$ mode. It can be seen that this mode has tangential magnetic fields at the boundaries of the cylindrical structure at $\theta=0^{\circ}$ and $\theta=180^{\circ}$. Thus if the resonator is excited at $\theta=0^{\circ}$ and the energy is coupled out at $\theta=180^{\circ}$, this mode can also be coupled out*. To prevent this, the resonator can be excited at $\theta=0^{\circ}$ and the output can be taken at $\theta=90^{\circ}$ or $270^{\circ}$ where the $\mathrm{H}$-field of the $\mathrm{TM}_{111}$ mode has zeros. This will ensure that only the energy of the $\mathrm{TE}_{011}$ mode will be coupled out and result in a large spurious free band.

The resonator can be excited by using a rectangular X-band waveguide operating in the dominant $\mathrm{TE}_{10}$ mode, rotated by $90^{\circ}$. This will ensure that the tangential $\mathrm{H}$-field components of the waveguide are parallel with the tangential $\mathrm{H}$-field components of the $\mathrm{TE}_{011}$ mode of the cylindrical resonator. Figure 3.18 shows the field patterns of the rectangular $\mathrm{TE}_{10}$ mode [24].

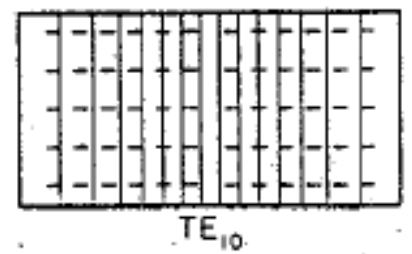

Figure 3.18: Field configuration of the rectangular $T E_{10}$ mode [24].

In order to obtain the correct coupling, the size of the rectangular coupling apertures must be the correct length and width and at the correct position (the external Q-factor must be equal to the unloaded Q-factor in order to obtain the correct coupling). This is done by simulating the structure lossless (the parallel combination of $R_{S}$ and $R_{L}$ of Figure 3.15 denotes the external loading of the resonator) and varying the widths and lengths of the apertures to find the ratio when $Q_{e x}=Q_{0}$ through a parameter sweep using the frequency domain solver in CST. The centre of the aperture is placed at the centre of the length of the cavity where the $\mathrm{H}$-field is the strongest. The depth of the aperture (and thus the minimum width of the cavity wall) was chosen as $3 \mathrm{~mm}$ to ensure that screws can be inserted at the top and bottom endplates and to connect the $\mathrm{X}$-band waveguide to the coupling ports of the cavity. The external Q-factor can be determined as

$$
Q_{e x}=\frac{f_{0}}{3 d B \text { Bandwidth }}
$$


Once the correct coupling is found, the structure can be simulated with the frequency domain solver in CST with the losses included. Figure 3.19 (a) and Figure 3.19 (b) shows the top and side view of the structure used in CST for the simulations, respectively.

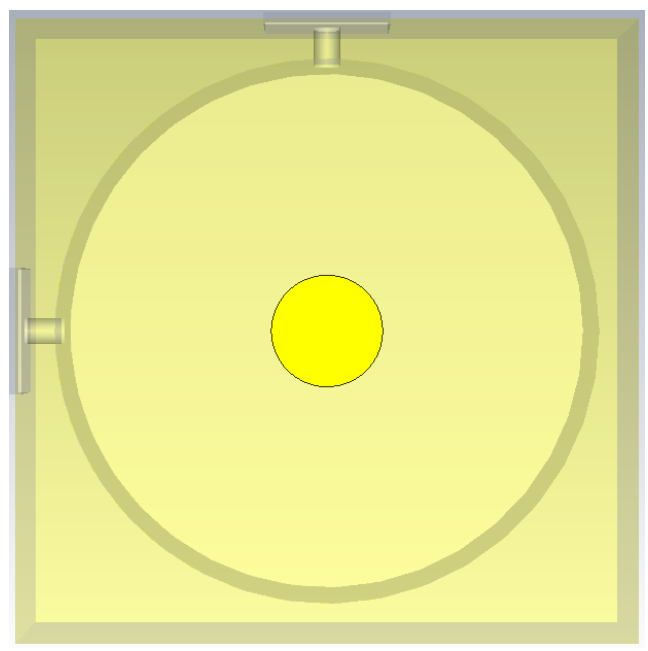

(a)

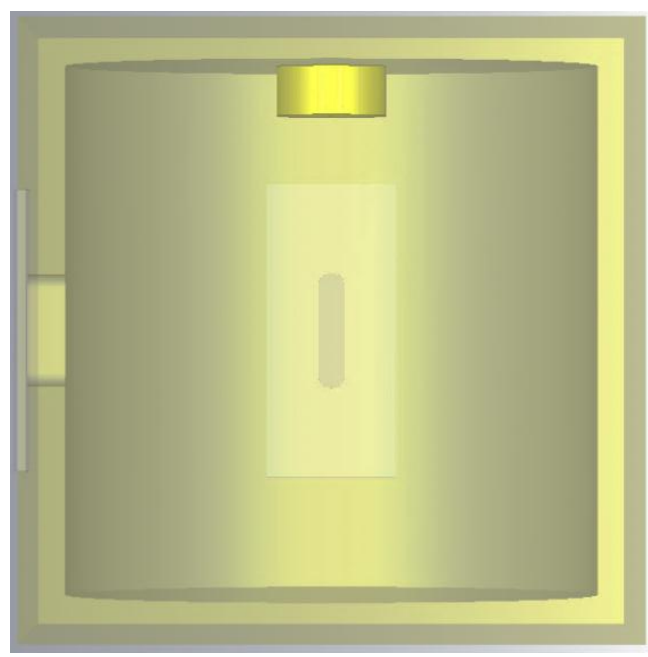

(b)

Figure 3.19: (a) Top and (b) side view of structure used in CST for the simulations.

The external Q-factor needed is obtained by use of (3.44) and the result is obtained from Figure 3.20 as

$$
\begin{aligned}
Q_{e x} & =\frac{9.200211 \mathrm{GHz}}{(9.2023-9.20192) \mathrm{GHz}} \\
& =24211 \\
& \approx Q_{0}
\end{aligned}
$$

\section{Notes $^{*}$}

- It should be noted that the apertures were first simulated as rectangular holes to obtain the correct coupling. The modelled internal corners of the apertures are thus 90 degrees, which is not easy to manufacture. After this approximation for the dimensions of the apertures were obtained, the internal corners were filleted with a radius of $1 \mathrm{~mm}$. Another parameter sweep in CST was thus necessary and the above external Q-factor was obtained.

- The height of the cavity was slightly reduced in order for the cavity's resonant frequency to be $9.2 \mathrm{GHz}$. The shift in resonant frequency is as a result of the introduction of the coupling apertures. The new height of the cavity is $h=42.7 \mathrm{~mm}$.

- Upon further inspection, it can be seen from Figure 3.6 (b) and from the fact that TM modes do not have any magnetic field components in the direction of propagation that the $\mathrm{TM}_{111}$ mode will not be excited nor coupled out with the coupling apertures used. If however some small amount of coupling is present due to e.g. asymmetry, it is good practice to suppress this mode to avoid any difficulties that may arise. This coupling scheme will also supress the $\mathrm{TE}_{311}$ mode and any mode whose $\mathrm{H}$-field has zeros at 90 degrees intervals and will be very beneficial in other applications. 
The dimensions of the apertures to obtain the correct coupling are

$$
\begin{gathered}
l=9 \mathrm{~mm} \\
w=2.1 \mathrm{~mm} \\
r=1 \mathrm{~mm}
\end{gathered}
$$

where $r$ is the fillet radius which the internal corners of the apertures are rounded.

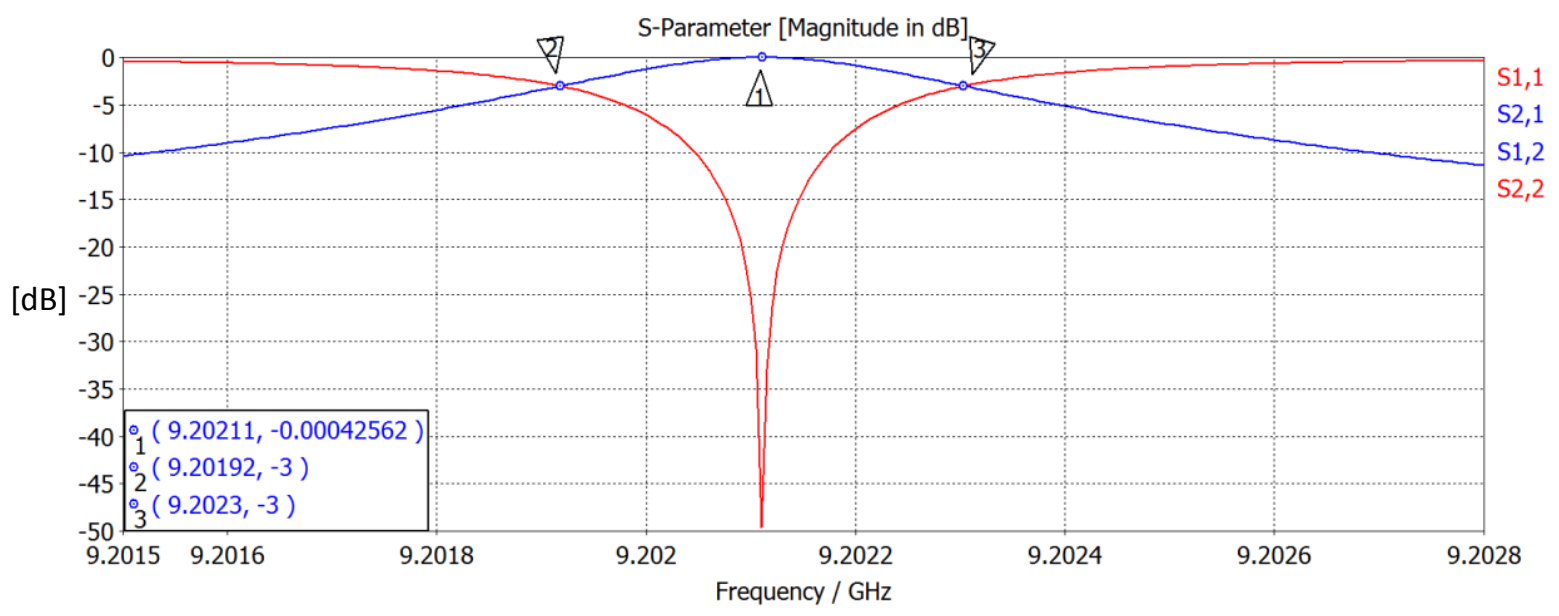

Figure 3.20: S-parameters obtained from a lossless CST simulation using the frequency domain solver.

Figure 3.21 shows the results obtained from simulating the structure with losses included and confirms the theoretical prediction that $Q_{l}=\frac{Q_{0}}{2}$ and $\left|S_{21}\right|_{\max }=\left|S_{11}\right|_{\min }=-6 d B$. Using (2.10),

$$
\begin{aligned}
Q_{l}= & \frac{9.20118 \mathrm{GHz}}{(9.20157-9.20077) \mathrm{GHz}} \\
& =11501 \\
& \approx \frac{Q_{0}}{2}
\end{aligned}
$$




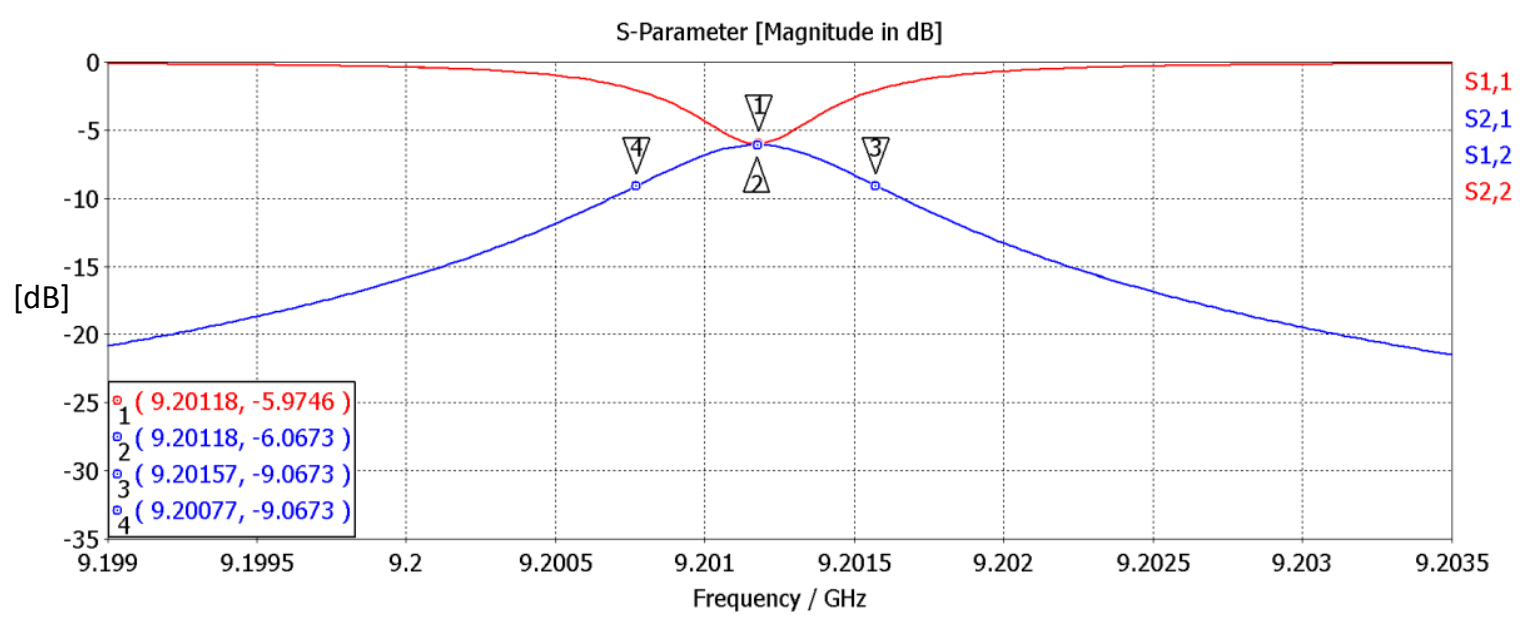

Figure 3.21: S-parameters obtained from a CST simulation using the frequency domain solver, with losses included.

\subsubsection{Tuning Range}

The perturbation screw also functions as a tuning mechanism. The minimum resonant frequency is obtained when the screw is tuned to its minimum $(l=0 \mathrm{~mm})$ and is shown in Figure 3.22. When the screw is tuned to its maximum value $(l=12 \mathrm{~mm})$, the highest resonant frequency is obtained, as shown in Figure 3.23.

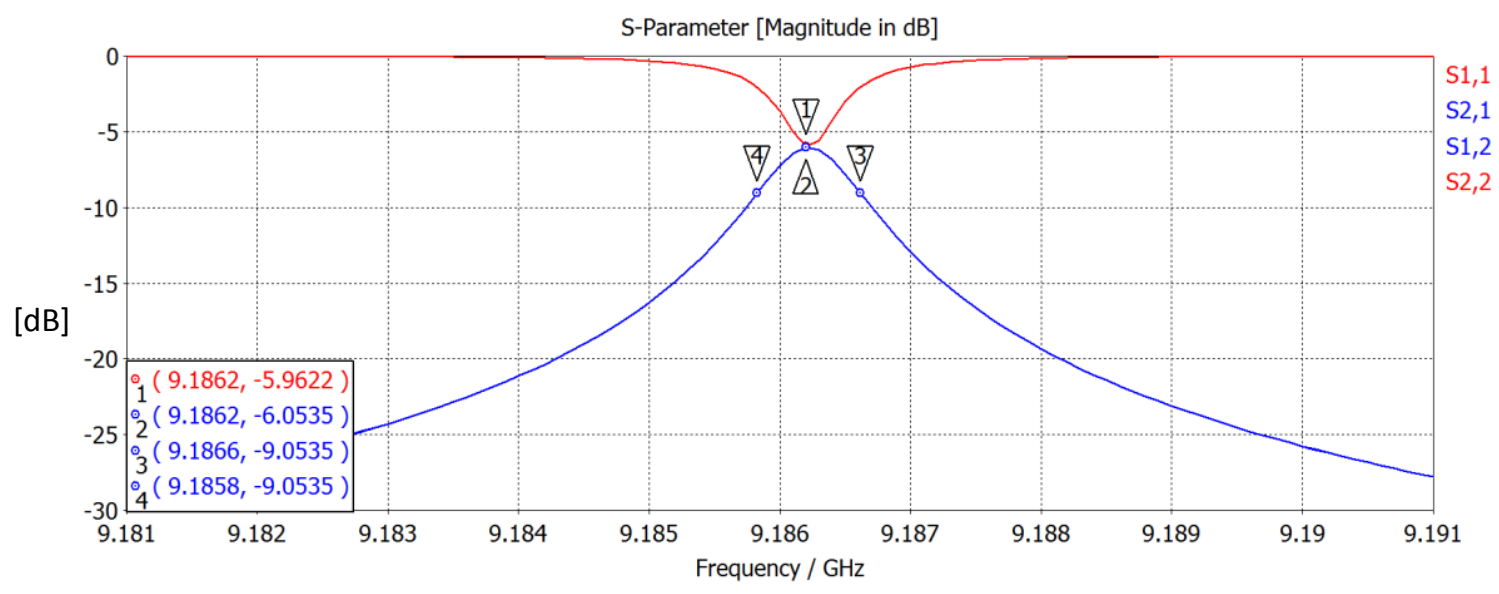

Figure 3.22: S-parameters of the low end of the tuning range of the resonator obtained from a CST simulation using the frequency domain solver, with losses included. The length of the tuning screw at the low end is at its minimum (zero). 


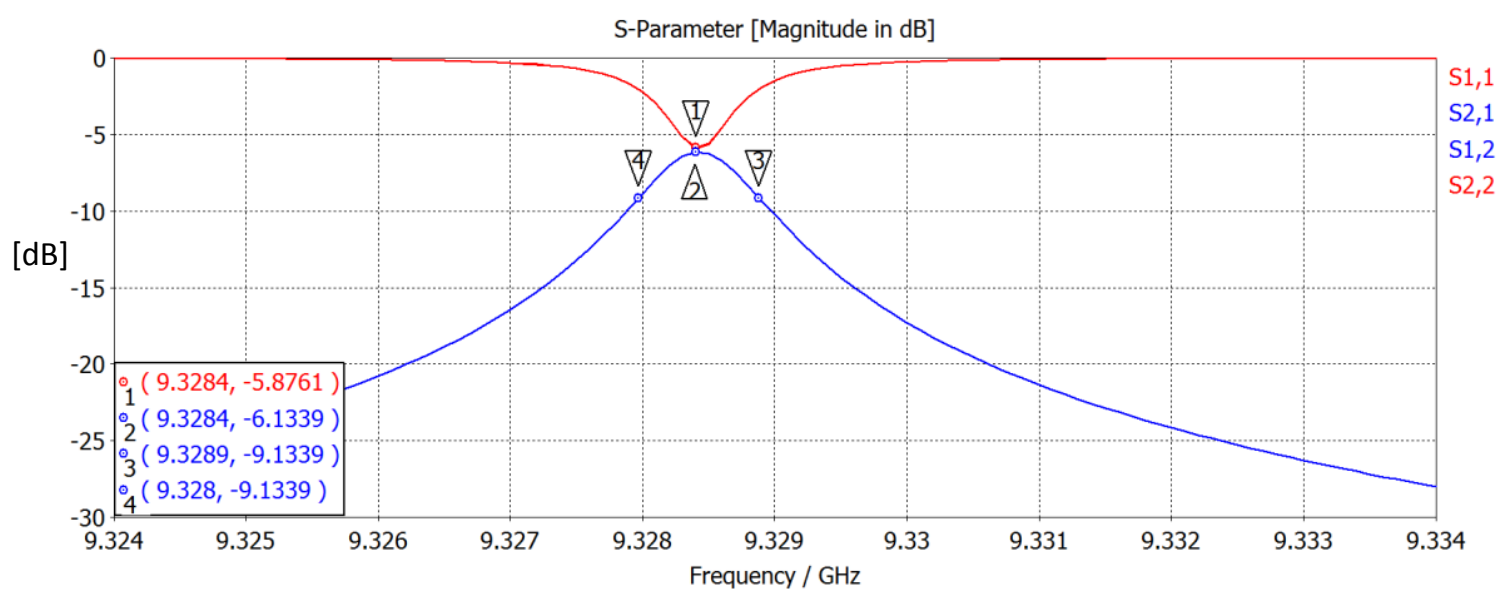

Figure 3.23: S-parameters of the high end of the tuning range of the resonator obtained from a CST simulation using the frequency domain solver, with losses included. The length of the tuning screw at the high end is at its maximum $(12 \mathrm{~mm})$.

The loaded and unloaded Q-factors of the lower and higher ends are

$$
\begin{aligned}
Q_{l} & =\frac{9.1862 \mathrm{GHz}}{(9.186619-9.185828) \mathrm{GHz}}=11613 \\
Q_{u} & =23494
\end{aligned}
$$

And

$$
\begin{aligned}
Q_{l} & =\frac{9.3284 \mathrm{GHz}}{(9.32888-9.32797) \mathrm{GHz}}=10250 \\
Q_{u} & =21959
\end{aligned}
$$

respectively.

As mentioned earlier, the $\mathrm{TE}_{011}$ mode has low losses when the screw is inserted into the structure. Tuning from the low end to the centre frequency ( $\approx 15 \mathrm{MHz}$ tuning) results in a lowering of the loaded Q-factor by $112(0.9 \%)$ and unloaded Q-factor by 611 . The loaded and unloaded Q-factor decreases by 1363 (11.7\%) and 1535, respectively, when tuning from the low end to the high end $(\approx$ $142 \mathrm{MHz}$ tuning). A wideband simulation is also performed in order to obtain all the spurious modes that are also coupled out, and is shown in Figure 3.24. 


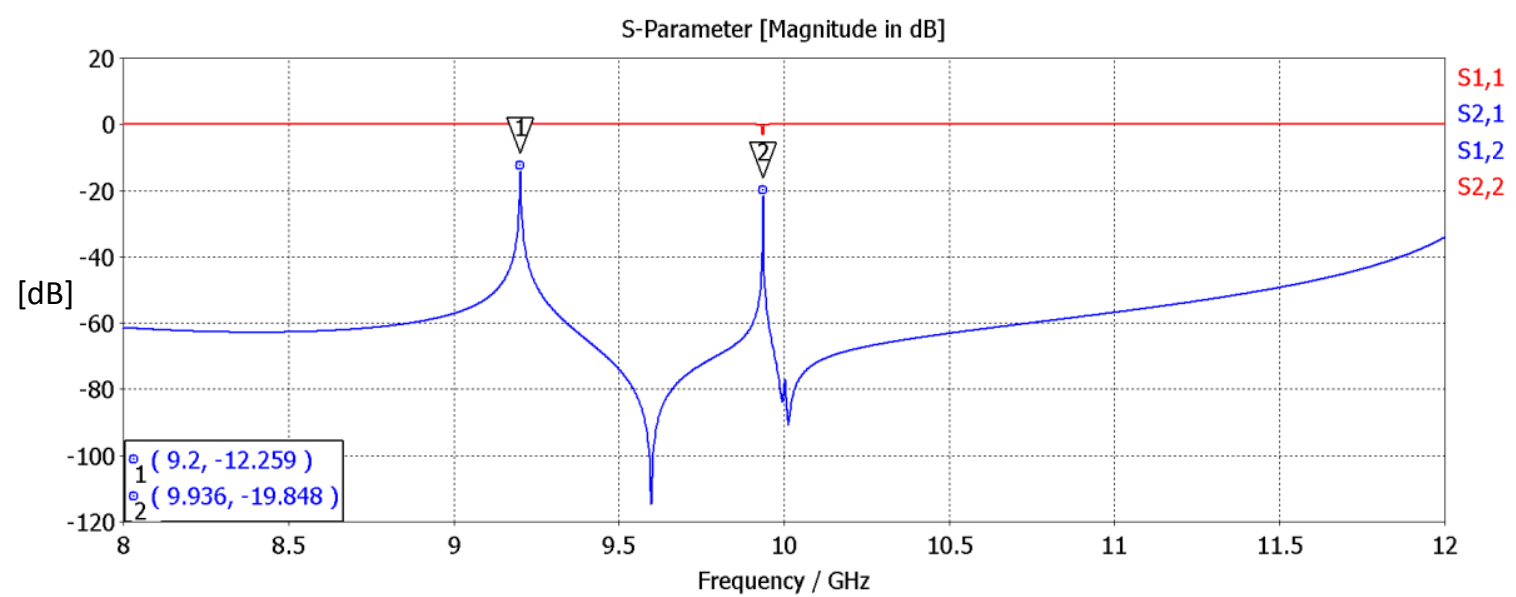

Figure 3.24: Wideband simulation of the resonator at its centre frequency obtained from a CST simulation.

\subsection{Filter}

A filter must be inserted in the oscillator loop in order to suppress spurious modes excited by the resonator and to avoid oscillations at different frequencies. A dual-mode rectangular waveguide cavity filter operating in the $\mathrm{TE}_{101}$ and $\mathrm{TE}_{011}$ modes is used to serve this purpose. Figure 3.25 (a) and Figure 3.25 (b) shows the field patterns of the $\mathrm{TE}_{10}$ and $\mathrm{TE}_{01}$ modes, respectively [24]. The solid lines represent the E-field and the dotted lines the $\mathrm{H}$-field.

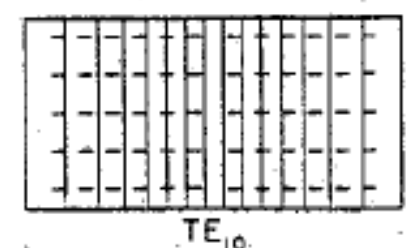

(a)

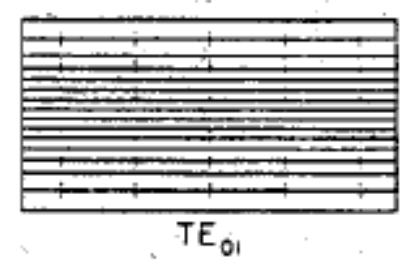

(b)

Figure 3.25: Field configurations of the rectangular (a) $T E_{10}$ and (b) $T E_{01}$ modes [24].

\subsubsection{Design}

\subsubsection{Cavity dimensions}

For a rectangular waveguide cavity, the cutoff and resonant frequencies of the TE modes are given by [23] as

$$
\begin{gathered}
\left(f_{c}\right)_{m n}=\frac{1}{2 \pi \sqrt{\mu \varepsilon}} \sqrt{\left(\frac{m \pi}{a}\right)^{2}+\left(\frac{n \pi}{b}\right)^{2}} \\
\left(f_{r}\right)_{m n p}^{T E}=\frac{1}{2 \pi \sqrt{\mu \varepsilon}} \sqrt{\left(\frac{m \pi}{a}\right)^{2}+\left(\frac{n \pi}{b}\right)^{2}+\left(\frac{p \pi}{c}\right)^{2}}
\end{gathered}
$$

For the cavity to operate in the $\mathrm{TE}_{101}$ and $\mathrm{TE}_{011}$ mode simultaneously, the dimensions in the $\mathrm{x}$ and $\mathrm{y}$ directions must be the same. This corresponds to $a=b$. Further, setting 


$\begin{array}{llll}\mu & & = & 4 \pi \mathrm{e}-7 \\ \varepsilon & & & 8.854 \mathrm{e}-12 \\ \mathrm{c} & = & \mathrm{a} & = \\ \left(f_{r}\right)_{101}^{T E}= & \left(f_{r}\right)_{011}^{T E} & = & 9.2 \mathrm{e} 9\end{array}$

and substituting these values into (3.53) and equating $a, b$ or $c$ from either $\left(f_{r}\right)_{011}^{T E}$ or $\left(f_{r}\right)_{101}^{T E}$, results that

$$
a=b=c=23.058 \mathrm{~mm}
$$

Using these dimensions in (3.52), the cutoff frequency of both modes is calculated as $6.5 \mathrm{GHz}$. It is thus evident that both modes are above cutoff at the operating frequency.

These dimensions will ensure that the $\mathrm{TE}_{101}$ and $\mathrm{TE}_{011}$ modes will resonate at the same frequency $(9.2 \mathrm{GHz})$, orthogonal to each other. Figure 3.26 (a) and Figure 3.26 (b) shows the E-field patterns, obtained from CST, of the $\mathrm{TE}_{101}$ and $\mathrm{TE}_{011}$ modes with the dimensions describes above, respectively.

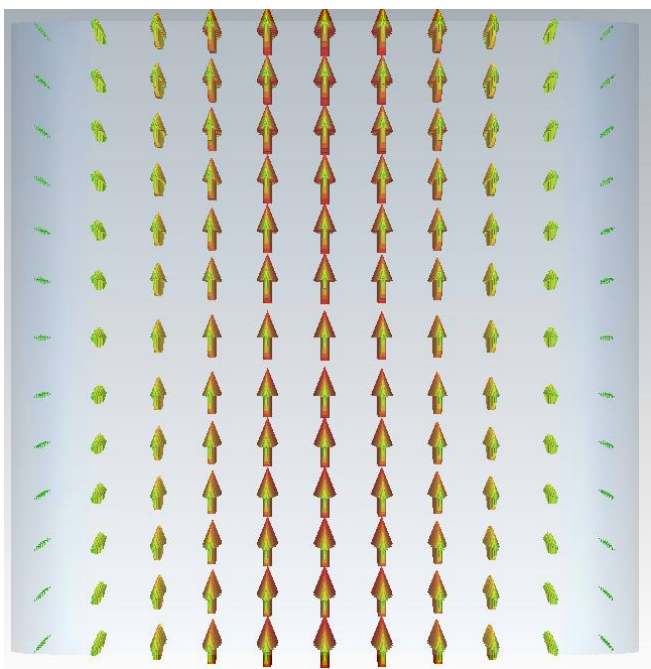

(a)

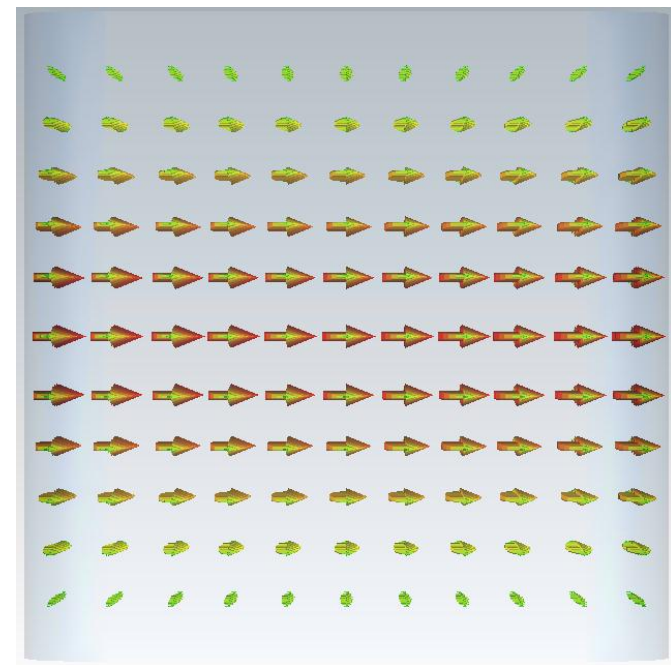

(b)

Figure 3.26: E-field patterns in the transverse direction of the rectangular (a) $T E_{101}$ and (b) $T E_{011}$ modes.

It is important to note that when the structure is manufactured, the internal corners of the cavity will not be exactly $90^{\circ}$, but will be filleted with a constant radius. It is thus necessary to compensate for these changes. In this case, the edges are filleted with a radius of $3 \mathrm{~mm}$. This smoothing does not negatively affect the operation of the filter and no compensation in cavity size is needed.

\subsubsection{Coupling}

A coupling screw is inserted as shown in Figure 3.27 in order to couple energy from the $\mathrm{TE}_{101}$ mode to the orthogonal $\mathrm{TE}_{011}$ mode, or vice versa.

Ramo [26] states that the excitation and reception of waves in a guide can be done by placing a probe in the direction of the electric field. Figure 3.27 shows the E-field patterns in the transverse plane of the orthogonal $\mathrm{TE}_{101}$ and $\mathrm{TE}_{011}$ modes. 


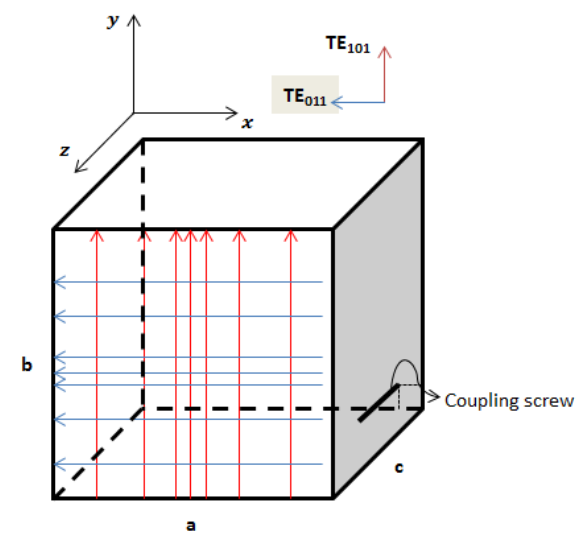

Figure 3.27: Structure illustrating the E-field patterns of the $T E_{101}$ and $T E_{011}$ modes. Also shown is the position of the coupling screw.

The positions of the maximum E-fields occur at $(x, y, z)=(a / 2, y, c / 2)$ for the $\mathrm{TE}_{101}$ mode and at $(x, y$, $z)=(x, b / 2, c / 2)$ for the $T E_{011}$ mode. Coupling energy in and out of the structure is thus done by inserting probes (SMA connectors) at $(x, y, z)=(a / 2, b, c / 2),(a, b / 2, c / 2)$, as shown in Figure 3.28. The lengths of these probes are chosen as $1 \mathrm{~mm}$.

In order to tune the centre frequency of the filter, tuning screws are inserted in the same plane as the probe (maximum E-field) on the opposite boundary, as illustrated in Figure 3.28. Inserting the screws deeper into the structure will cause a lowering in resonant frequency of the particular mode, as explained in section 3.1.2.1. Inserting the coupling screw deeper into the structure will result in stronger coupling between the orthogonal modes.

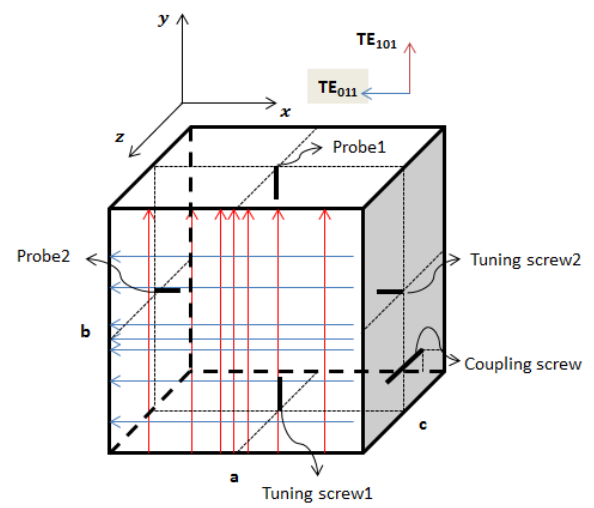

Figure 3.28: Structure illustrating the positions of the input and output probes. Also shown are the tuning screws for the two modes.

\subsubsection{Simulations}

The coupling and tuning screws used for the simulations and which will be used in the manufactured structure have a diameter of $3.2 \mathrm{~mm}$. In order to test if the filter will work correctly, the structure is first simulated lossless with a parameter sweep using the frequency domain solver in CST, by varying the lengths of the coupling and tuning screws. Figure 3.29 shows the model used in CST and Figure 3.30 shows the results when the filter is tuned to the centre frequency of $9.2 \mathrm{GHz}$ with a bandwidth of $12 \mathrm{MHz}$, respectively. The values of the parameters used to obtain these results are presented in 
Table 3-1. The coupling screw was chosen as the Temex 6925-3 and the Temex 6925-0 screw was chosen for both the tuning screws. The datasheet of these screws are available online at http://www.temex-ceramics.com/site/fichiers/DatasheetTuning.pdf.

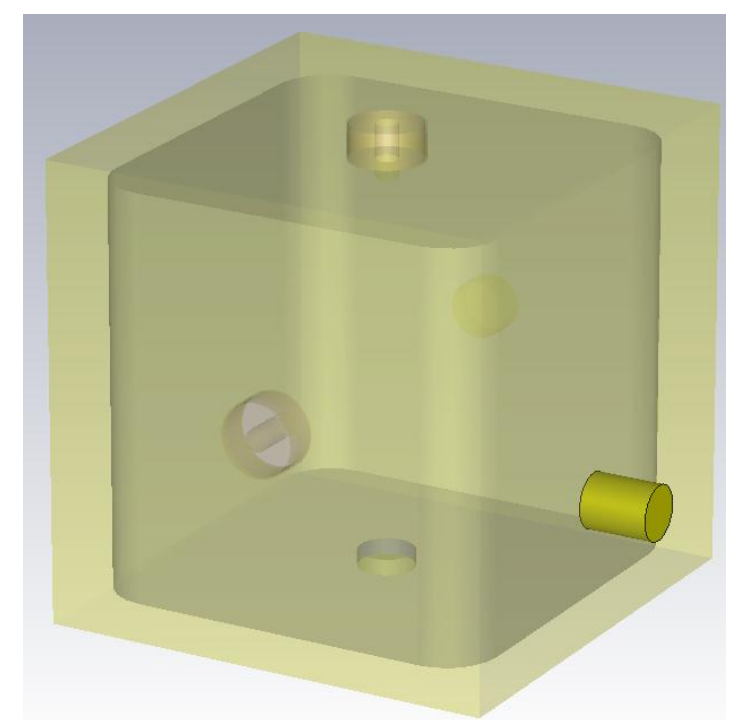

Figure 3.29: Filter structure used in CST for simulations.

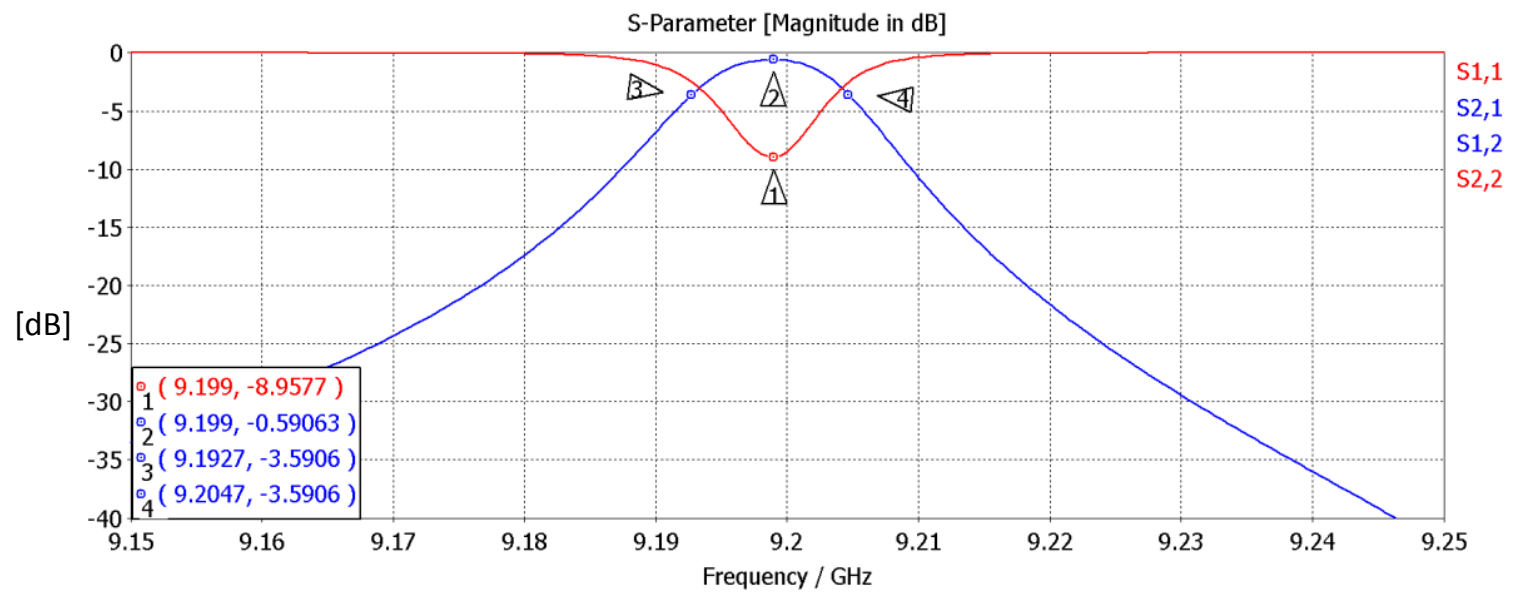

Figure 3.30: S-parameters of the filter obtained from a lossless CST simulation using the frequency domain solver.

Table 3-1: Parameter values used to generate Figure 3.30 and Figure 3.31.

\begin{tabular}{|l|l|}
\hline Parameter & Value [mm] \\
\hline Length of sides of cavity: $a, b, c$ & 23 \\
\hline Probe length 1 & 1 \\
\hline Probe length 2 & 1 \\
\hline Tuning screw length 1 & 1.6 \\
\hline Tuning screw length 2 & 0.9 \\
\hline Coupling screw & 4 \\
\hline
\end{tabular}

Next, the structure is simulated with the parameters in Table 3-1 with losses included, using the frequency domain solver in CST. The magnitude of the S-parameters is shown in Figure 3.31. 


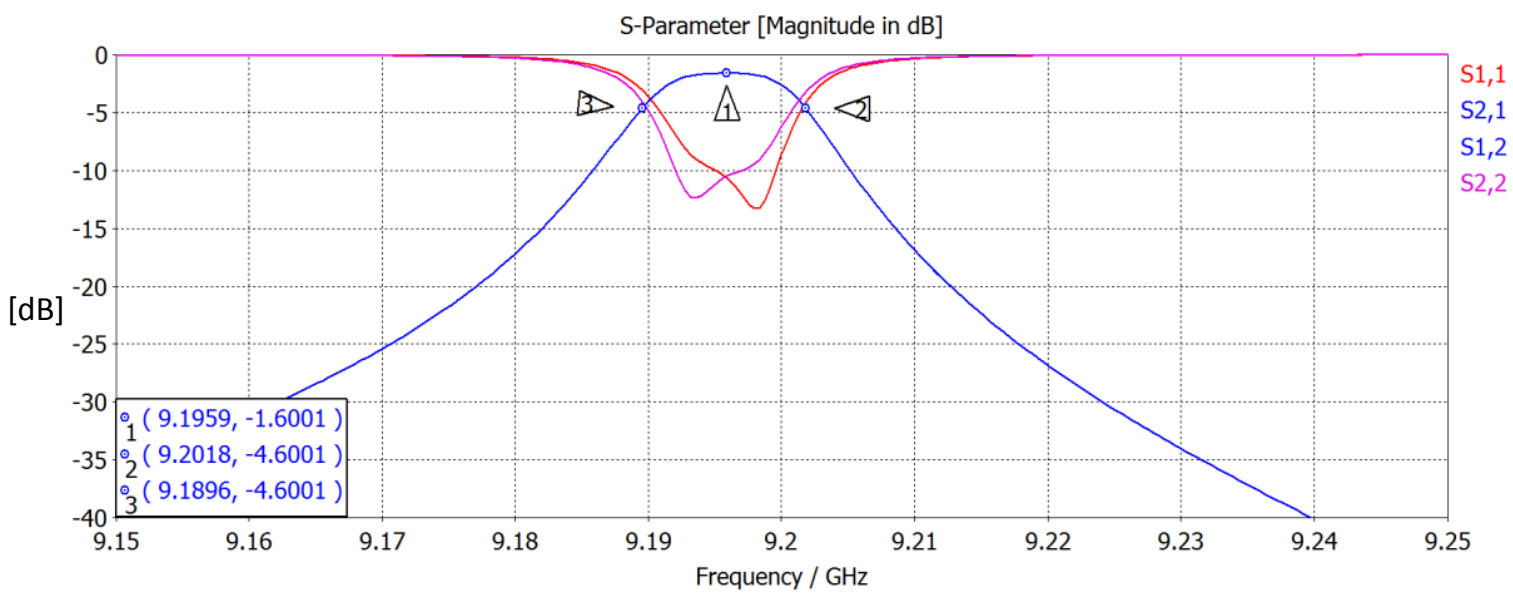

Figure 3.31: S-parameters of the filter obtained from a CST simulation using the frequency domain solver, with losses included.

The filter has a bandwidth of approximately $12 \mathrm{MHz}$, an insertion loss of $1.6 \mathrm{~dB}$ and a return loss of approximately $10 \mathrm{~dB}$. These results are satisfactory, as the goal of the filter is to suppress any spurious modes excited in the resonator while a small insertion loss is not critical.

A wideband simulation is also performed to investigate spurious pass-bands. The result is shown in Figure 3.32 .

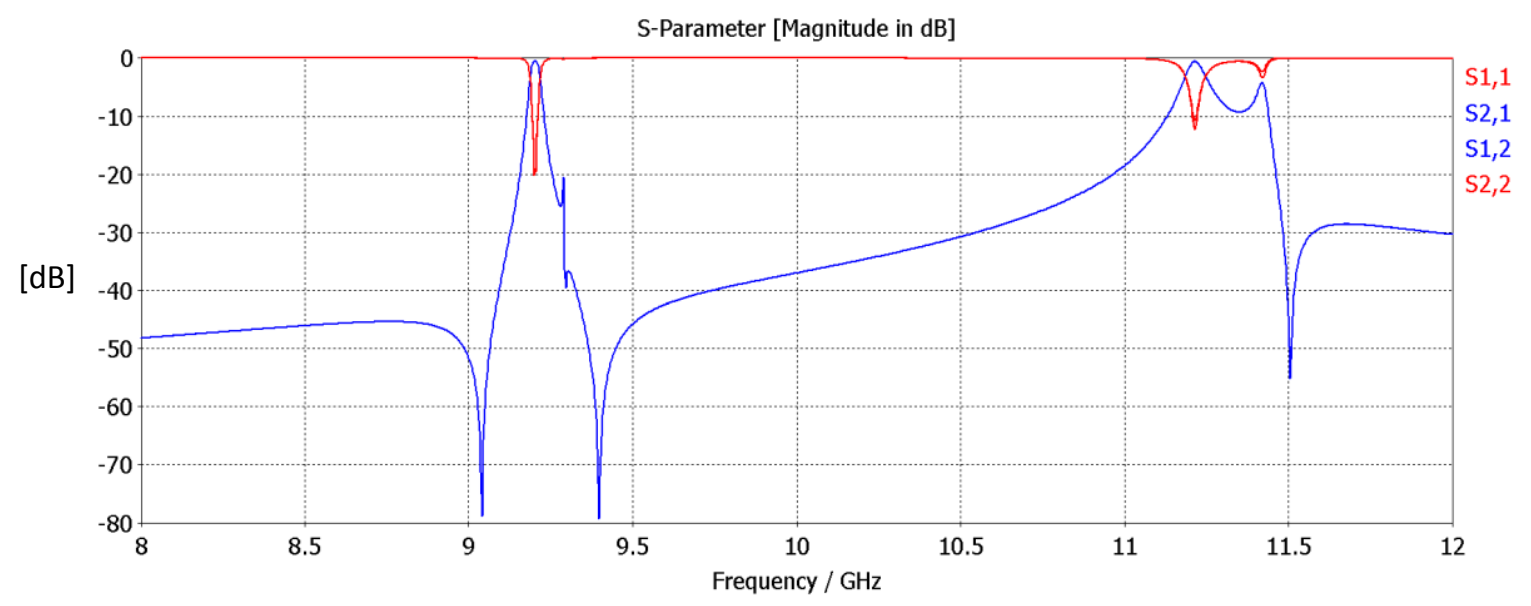

Figure 3.32: Wideband simulation of the filter at its centre frequency obtained from a CST simulation.

The touchstone files of the resonator and filter are exported from CST and the cascaded section is simulated in MWO. In order to avoid coupling between the resonator and filter, an attenuator is inserted between them to allow the resonator to see a resistive load, as shown in Figure 3.33. 


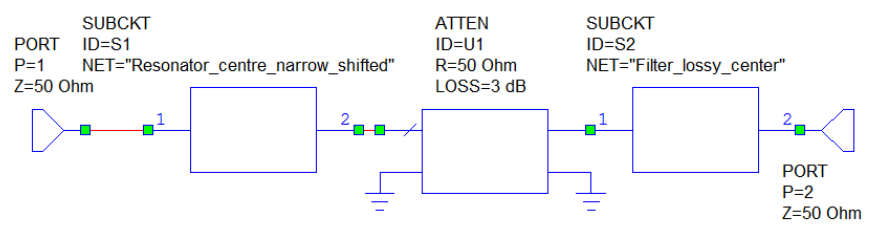

Figure 3.33: Schematic used in MWO of resonator and filter cascaded with a 3 dB attenuator between them.

Figure 3.34 shows the results of the resonator and filter connected directly to each other and with a $3 \mathrm{~dB}$ attenuator inserted between them.

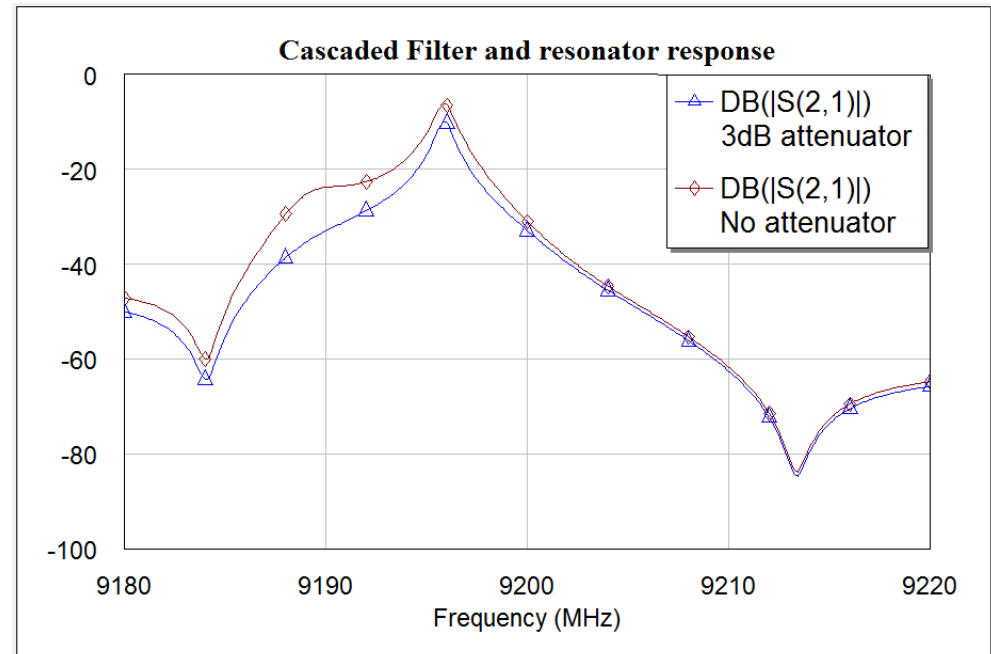

Figure 3.34: Simulation obtained from MWO illustrating the response of the cascade with and without the attenuator.

It is clear from the figure that there is coupling between the filter and the resonator and the system's performance is degraded when the attenuator is absent. 


\section{Chapter 4}

\section{Measurements}

This chapter presents and describes all the necessary measurements done on each component and on the whole system. The phase noise performance of the oscillator designed in this thesis will also be compared to various oscillators at the end of this chapter. The equipment used for the measurements are listed in Table 4-1.

Table 4-1: Equipment used for the measurements performed in this section.

\begin{tabular}{|l|l|}
\hline Measuring Device & Model \\
\hline Vector Network Analyser (VNA) & HP 8510 \\
\hline Spectrum Analyser (SA) & R\&S ${ }^{\circledR}$ FSEK 30 \\
\hline Signal Source Analyser & R\&S $^{\circledR}$ FSUP-B60 \\
\hline
\end{tabular}

\subsection{Resonator}

The cavity is constructed from aluminium with an air dielectric. The CAD drawing used for the manufacturing of the structure is inserted in Appendix A.

The resonator is first measured before being silver plated in order to investigate the improvement the plating has on the loaded Q-factor. Figure 4.1 shows the physical resonator used in these measurements and in the oscillator. 
Figure 4.1: Tuneable cylindrical waveguide cavity resonator operating in the $T E_{011}$ mode with centre frequency at $9.2 \mathrm{GHz}$.

The resonator with the waveguide to SMA transitions and the top endplate disconnected from the cavity is shown in Figure 4.2.

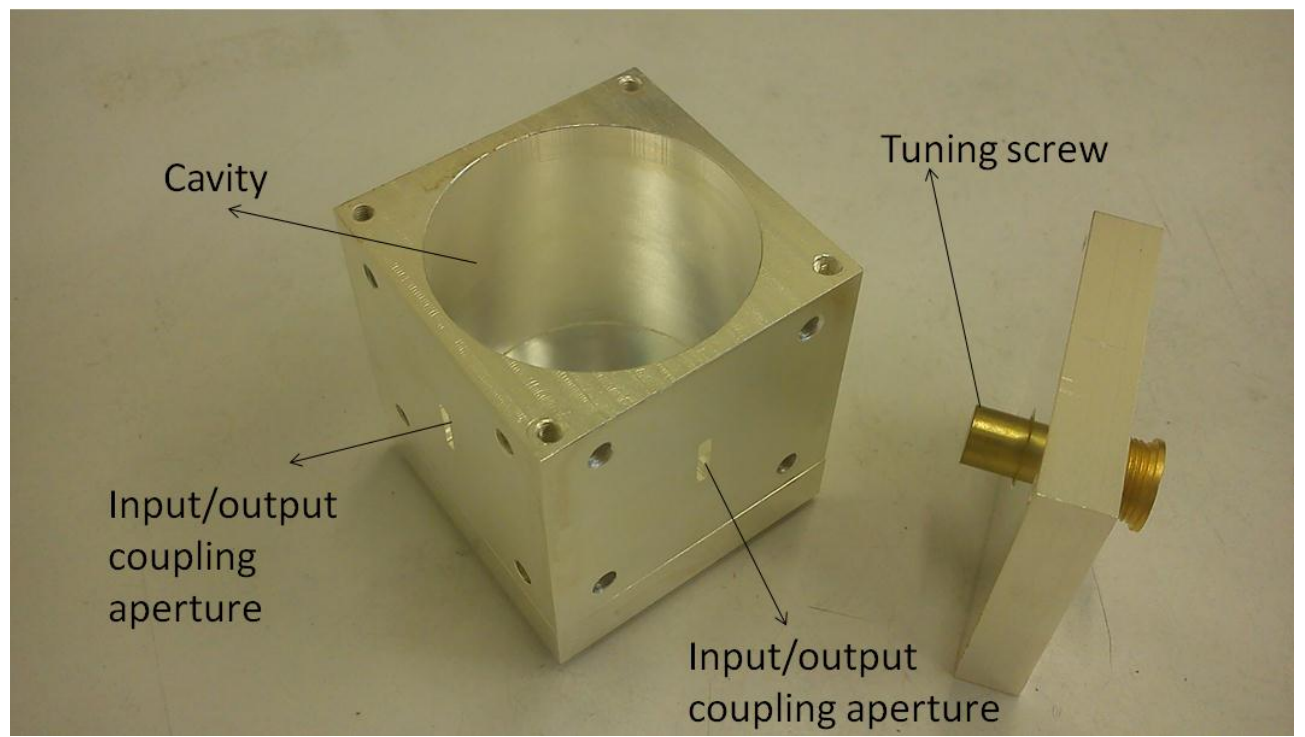

Figure 4.2: Resonator with waveguide transitions and top endplate disconnected.

Measurements are made in a narrow band $(20 \mathrm{MHz})$ at the centre frequency, as well at the minimum and maximum tuning frequencies. A broadband measurement over the band of 8 to 12.4 $\mathrm{GHZ}$ is also performed. These measurements are done using the HP8510 VNA and the data are extracted using MATLAB. 


\subsubsection{Tuning range of unplated resonator}

Figure 4.3 to Figure 4.5 shows the S-parameters of the unplated resonator at its low-end, centre frequency and high-end.
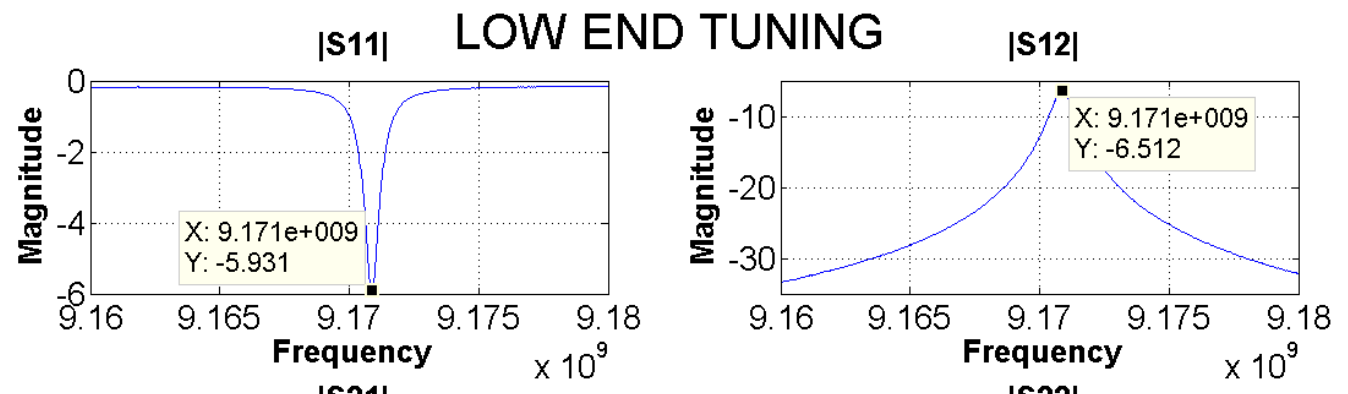

|S21|
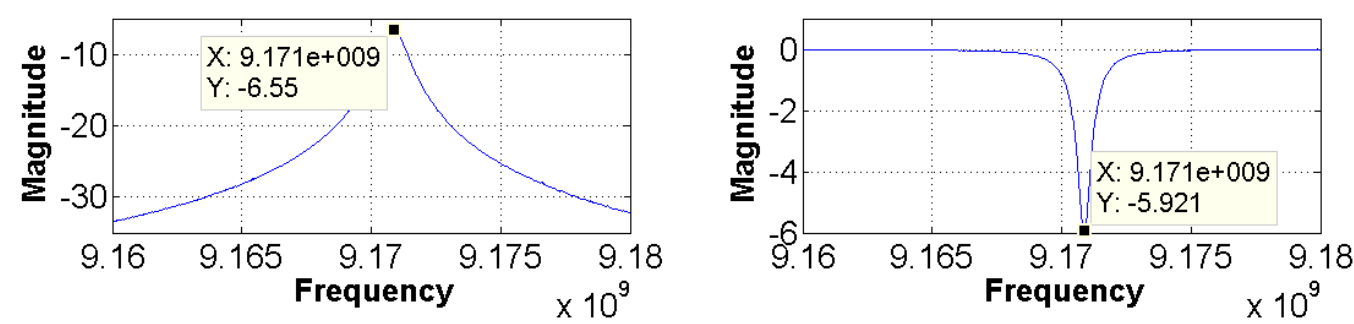

Figure 4.3: Measured S-parameters of resonator tuned to its lowest operating frequency.

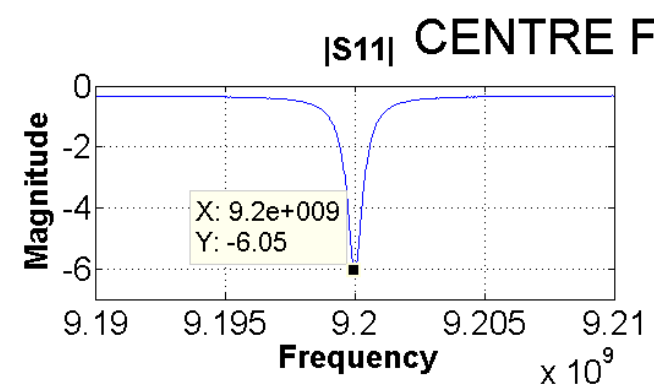

|S21|

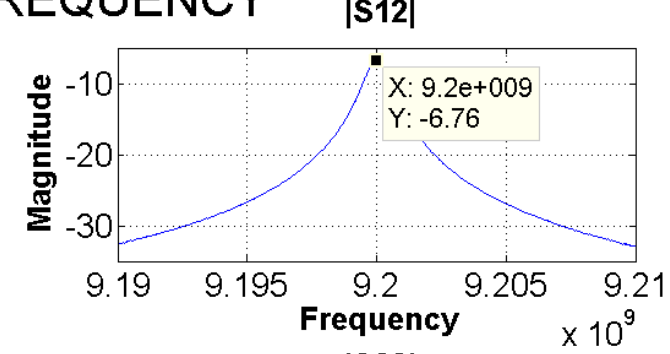

|S22|
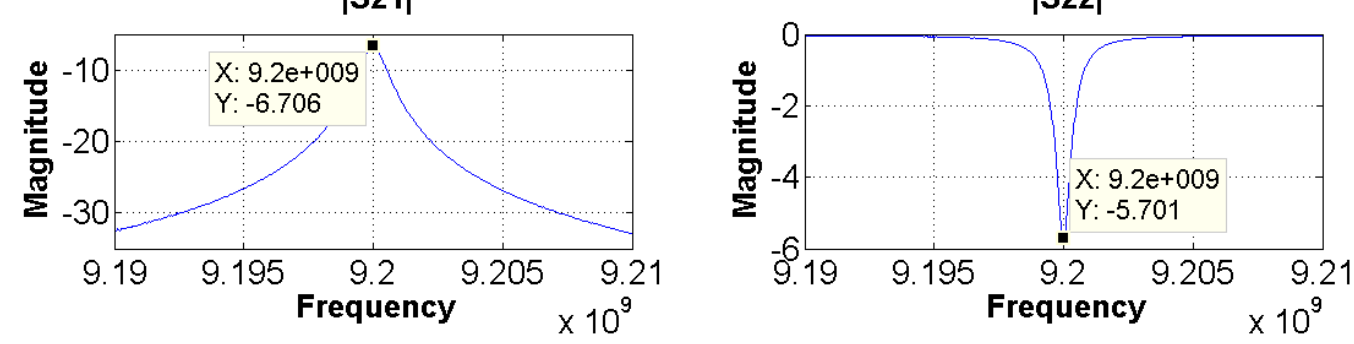

Figure 4.4: Measured S-parameters of resonator tuned to its centre frequency. 


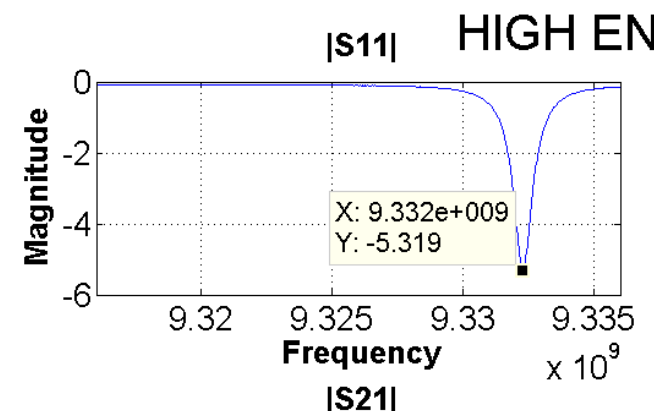

\section{TUNING}

|S12|
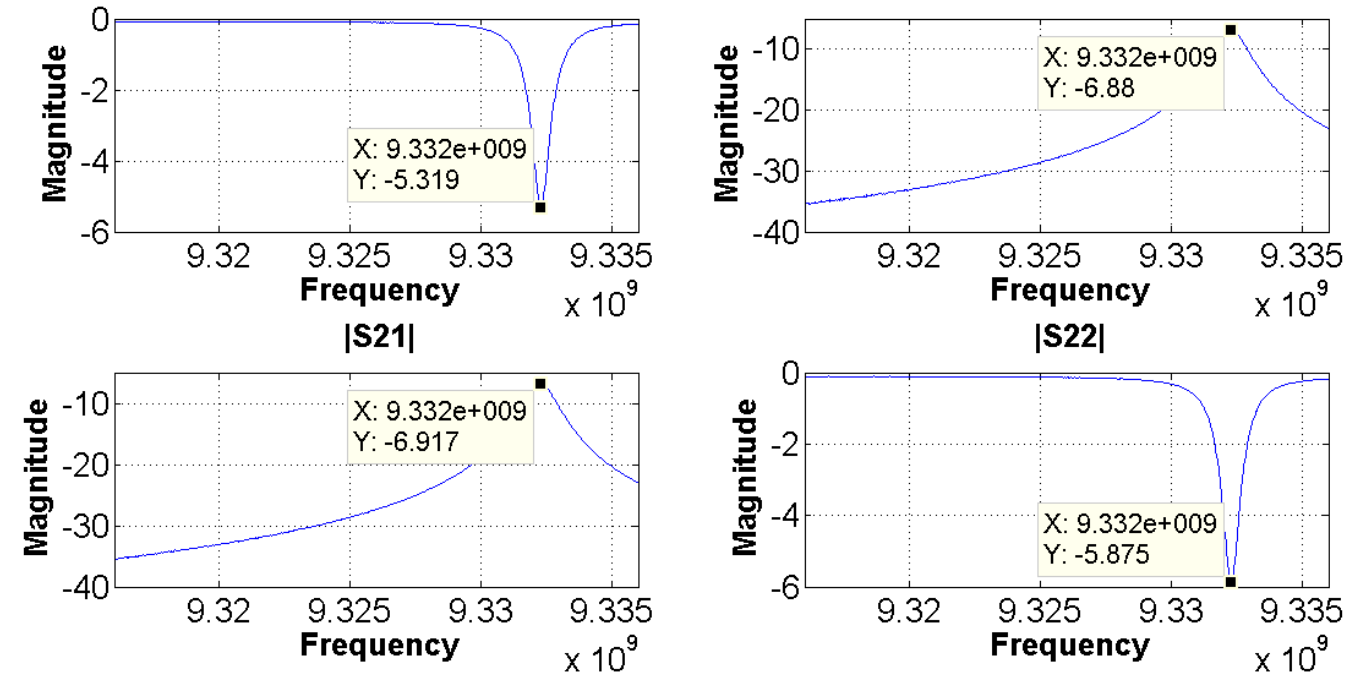

Figure 4.5: Measured S-parameters of resonator tuned to its highest operating frequency.

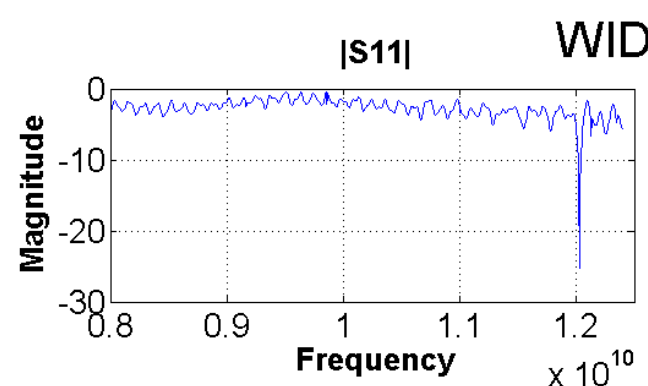

|S21|
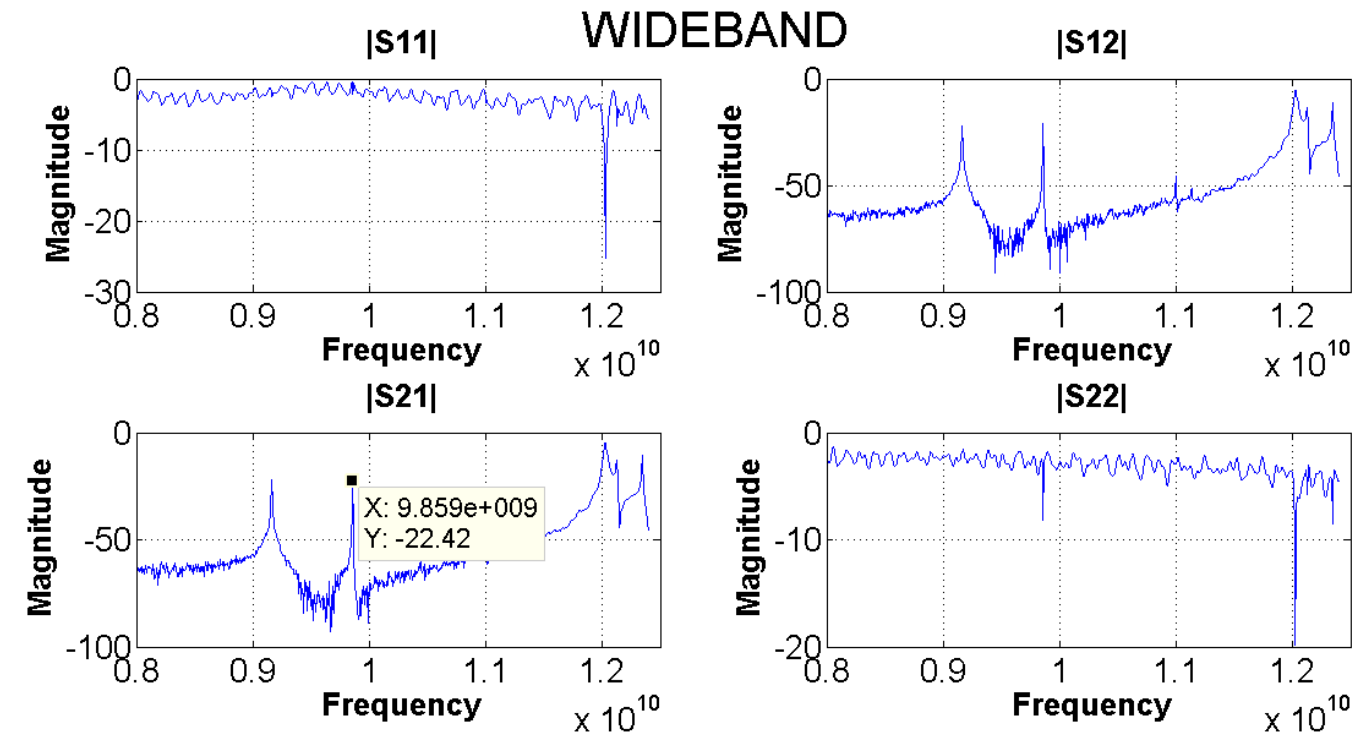

|S22|

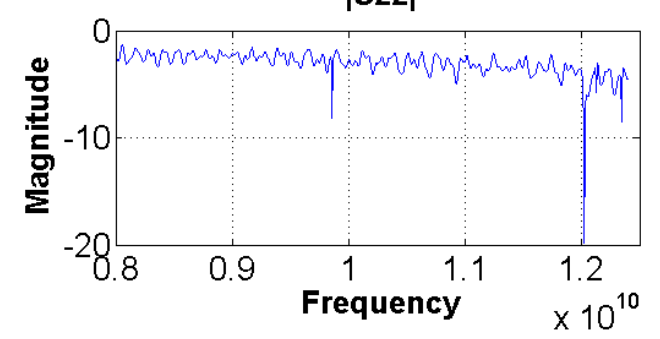

Figure 4.6: Wideband measurement of resonator tuned to its centre frequency.

The spurious modes can be seen from the wideband measurement in Figure 4.6. The first spurious mode occurs at approximately $9.8 \mathrm{GHz}$, which agrees with the frequency of the predicted $\mathrm{TE}_{212}$ mode. It is interesting to note the absence of the $\mathrm{TM}_{111}$ and the $\mathrm{TE}_{311}$ modes, which are due to the direction of the $\mathrm{H}$-field components in the input and output coupling apertures and the angle of coupling to the resonator. From $12 \mathrm{GHz}$ onwards, the presence of the $\mathrm{TM}_{013}, \mathrm{TM}_{211}$ and $\mathrm{TE}_{411}$ modes can clearly be seen.

The measured loaded Q-factor of the resonator at its minimum-, maximum- and centre frequencies are listed in Table 4-2 along with the theoretical values. 
Table 4-2: Simulated and measured loaded Q-factor of the resonator at its lowest-, centre- and highest operating frequency.

\begin{tabular}{|l|l|l|l|l|}
\hline \multirow{2}{*}{} & \multicolumn{2}{|c|}{ Frequency $(\mathrm{GHz})$} & \multicolumn{2}{c|}{ Loaded Q-factor } \\
\cline { 2 - 5 } & \multicolumn{1}{|c|}{ Theoretical } & \multicolumn{1}{c|}{ Measured } & \multicolumn{1}{c|}{ Theoretical } & Measured \\
\hline Low end & 9.1862 & 9.170875 & 11613 & 9474 \\
\hline Centre & 9.2 & 9.2 & 11501 & 9213 \\
\hline High end & 9.3284 & 9.33275 & 10250 & 7916 \\
\hline
\end{tabular}

\subsubsection{Tuning range of silver plated resonator}

In order to further increase the Q-factor of the resonator, it is plated with a $10 \mu \mathrm{m}$ layer of silver. Again, the resonator is measured in a narrow band at the minimum and maximum tuning frequencies and at the centre frequency, using the HP8510 VNA. The S-parameters obtained from these measurements are shown from Figure 4.7 to Figure 4.9.
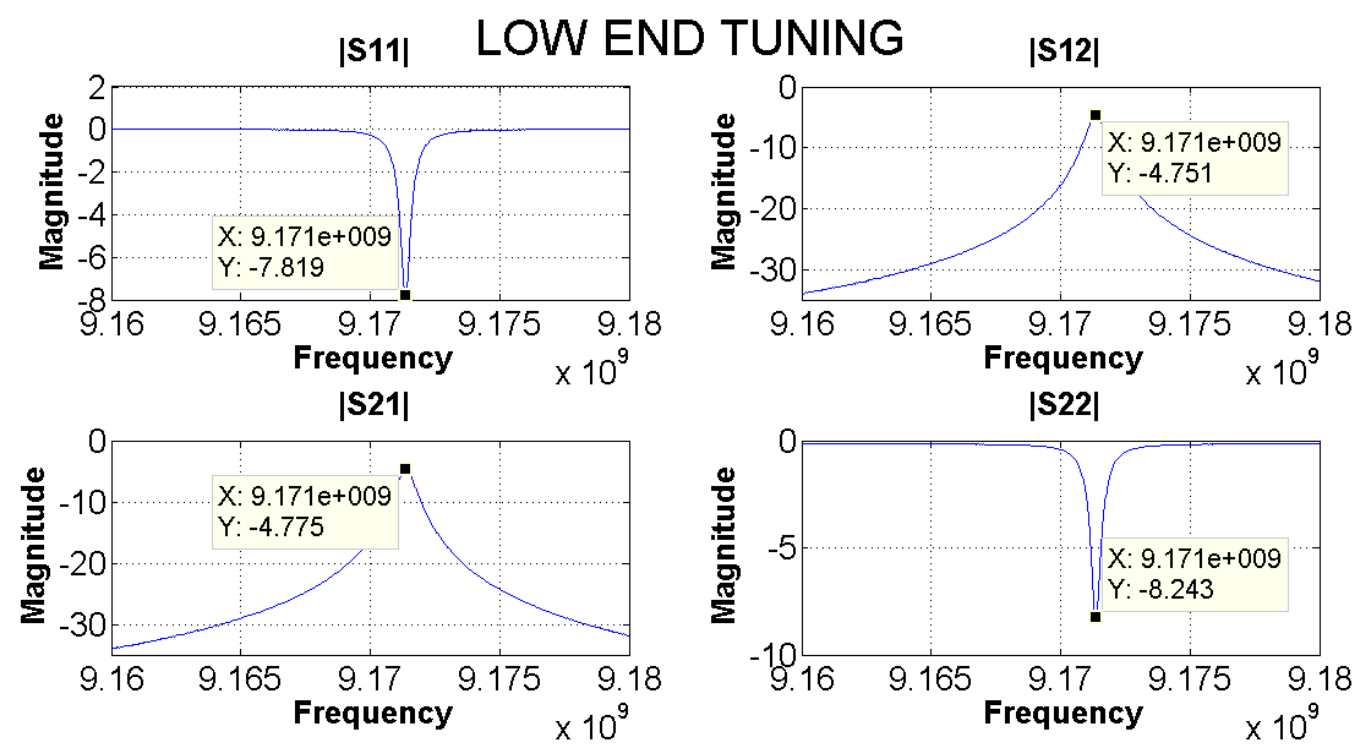

Figure 4.7: Measured S-parameters of resonator plated with silver, tuned to its lowest operating frequency. 


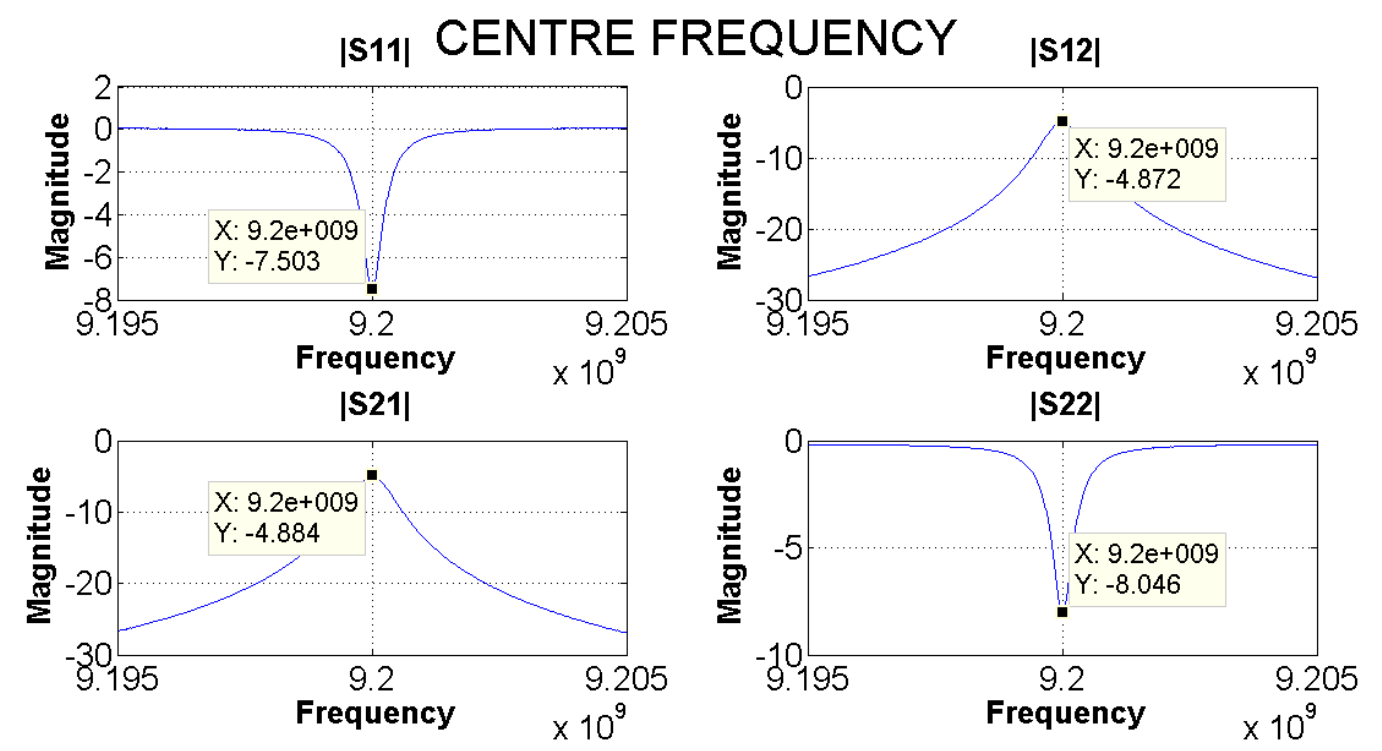

Figure 4.8: Measured S-parameters of resonator plated with silver, tuned to its centre frequency.
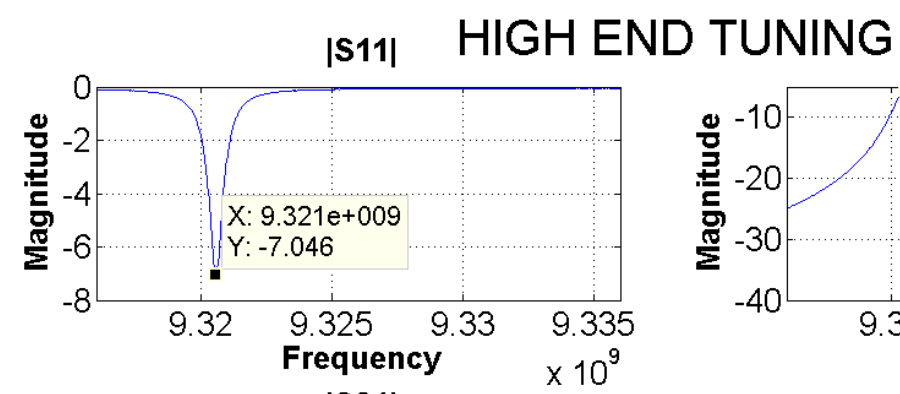

|S12|
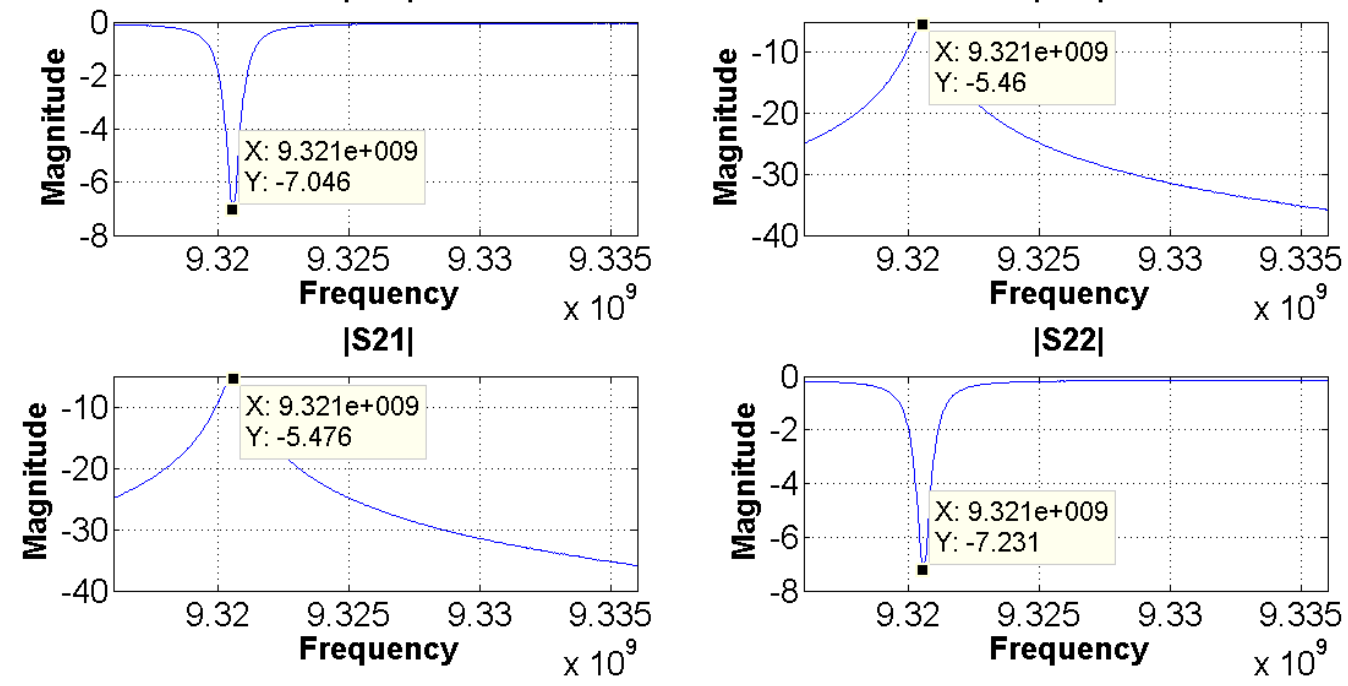

|S22|

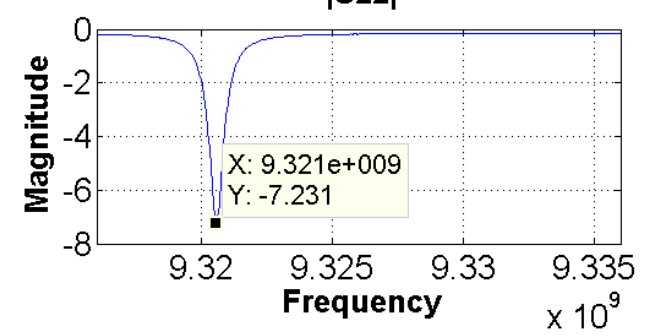

Figure 4.9: Measured S-parameters of resonator plated with silver, tuned to its lowest operating frequency.

The loaded Q-factors at the different frequencies are listed in Table 4-3, along with the unplated Q-factors and the theoretical values.

Table 4-3: Theoretical and measured loaded Q-factors of the resonator, with and without silver plating.

\begin{tabular}{|l|l|l|l|l|l|l|}
\hline \multirow{2}{*}{} & \multicolumn{3}{|c|}{ Frequency (GHz) } & \multicolumn{3}{c|}{ Loaded Q-factor } \\
\cline { 2 - 7 } & Theoretical & $\begin{array}{c}\text { Measured } \\
\text { (unplated) }\end{array}$ & $\begin{array}{c}\text { Measured } \\
\text { (plated) }\end{array}$ & Theoretical & $\begin{array}{c}\text { Measured } \\
\text { (unplated) }\end{array}$ & $\begin{array}{c}\text { Measured } \\
\text { (plated) }\end{array}$ \\
\hline Low end & 9.1862 & 9.170875 & 9.171375 & 11613 & 9474 & 11912 \\
\hline Centre & 9.2 & 9.2 & 9.2 & 11501 & 9213 & 11467 \\
\hline High end & 9.3284 & 9.33275 & 9.32055 & 10250 & 7916 & 9638 \\
\hline
\end{tabular}


It can be seen from Table 4-3 that the loaded Q-factor of the resonator at its centre frequency has increased by 2254 ( $24.5 \%$ ) after being plated with silver and agrees very well with the loaded Qfactor obtained from the CST simulation.

\subsection{Filter}

The filter, shown in Figure 4.10, is constructed from aluminium and the CAD drawing used for the manufacturing is inserted in Appendix B. The filter is also measured by using the HP8510 VNA and the data is exported to MATLAB.

Figure 4.10: Tuneable rectangular waveguide cavity dual mode filter operating in the $T E_{101}$ and $T E_{011}$ modes with centre frequency at $9.2 \mathrm{GHz}$.

Figure 4.11 shows a top view of the filter with the top endplate disconnected from the cavity in order to illustrate all the coupling and tuning mechanisms.

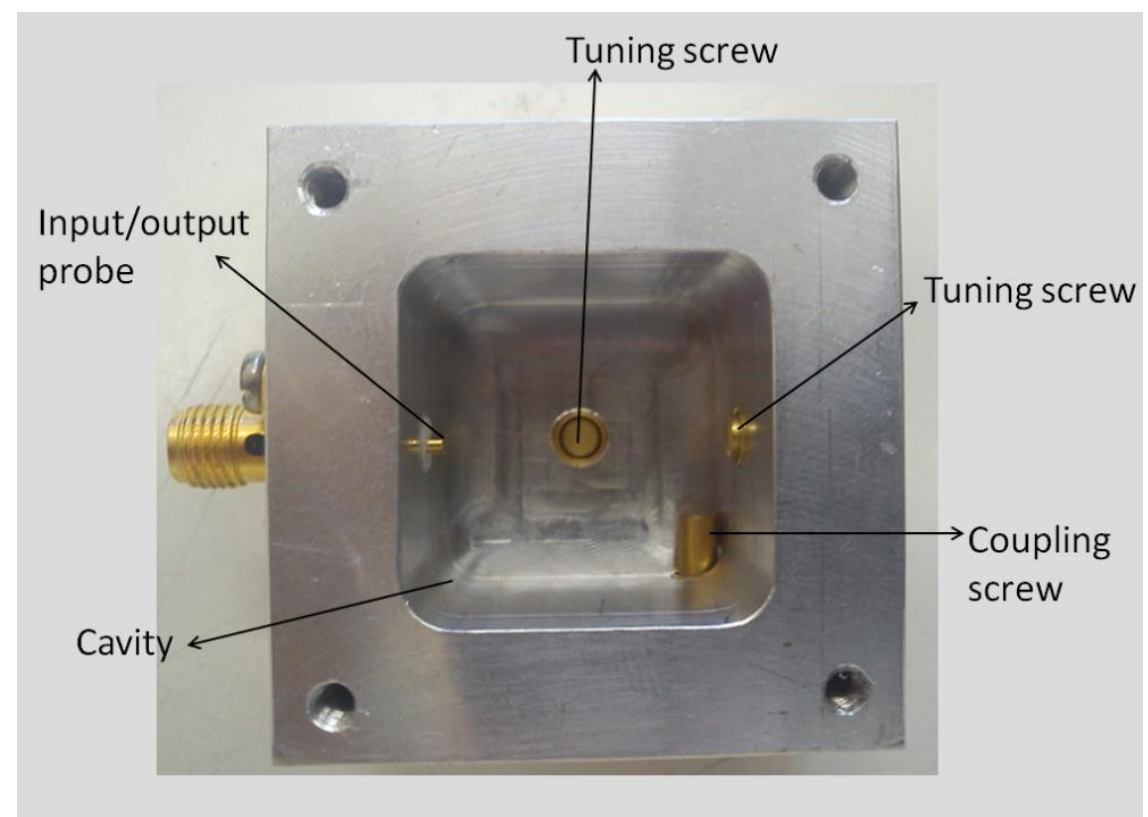

Figure 4.11: Filter with top endplate removed. 


\subsubsection{Tuning range}

Should it be decided that the oscillator must operate at a different frequency, it will be limited by the tuning ranges of the resonator and the filter. Measurements of the low- and high end of the tuning range and of the centre frequency are made. These measurements are done over a bandwidth of $300 \mathrm{MHz}$. A broadband measurement from 8 to $12 \mathrm{GHz}$ is also included. Figure 4.12 to Figure 4.15 shows these measurements.
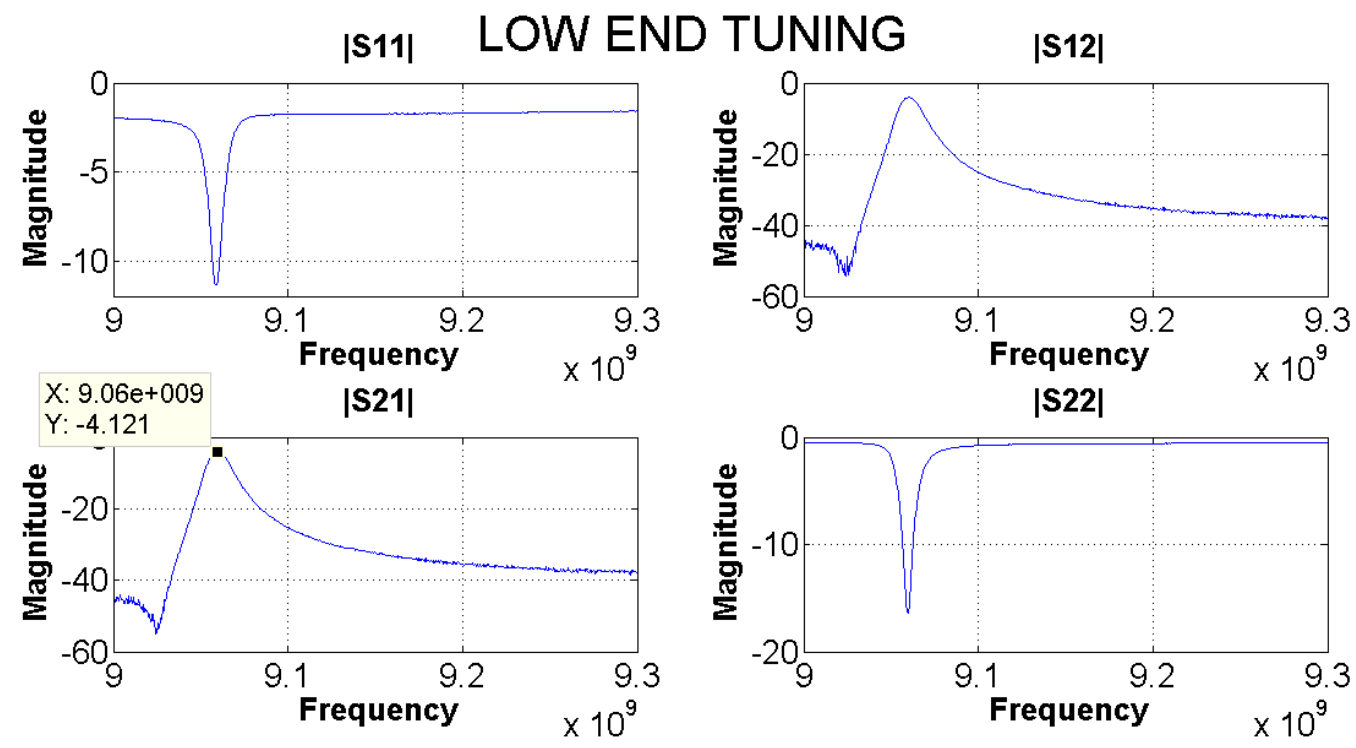

Figure 4.12: Measured S-parameters of filter tuned to its lowest operating frequency.
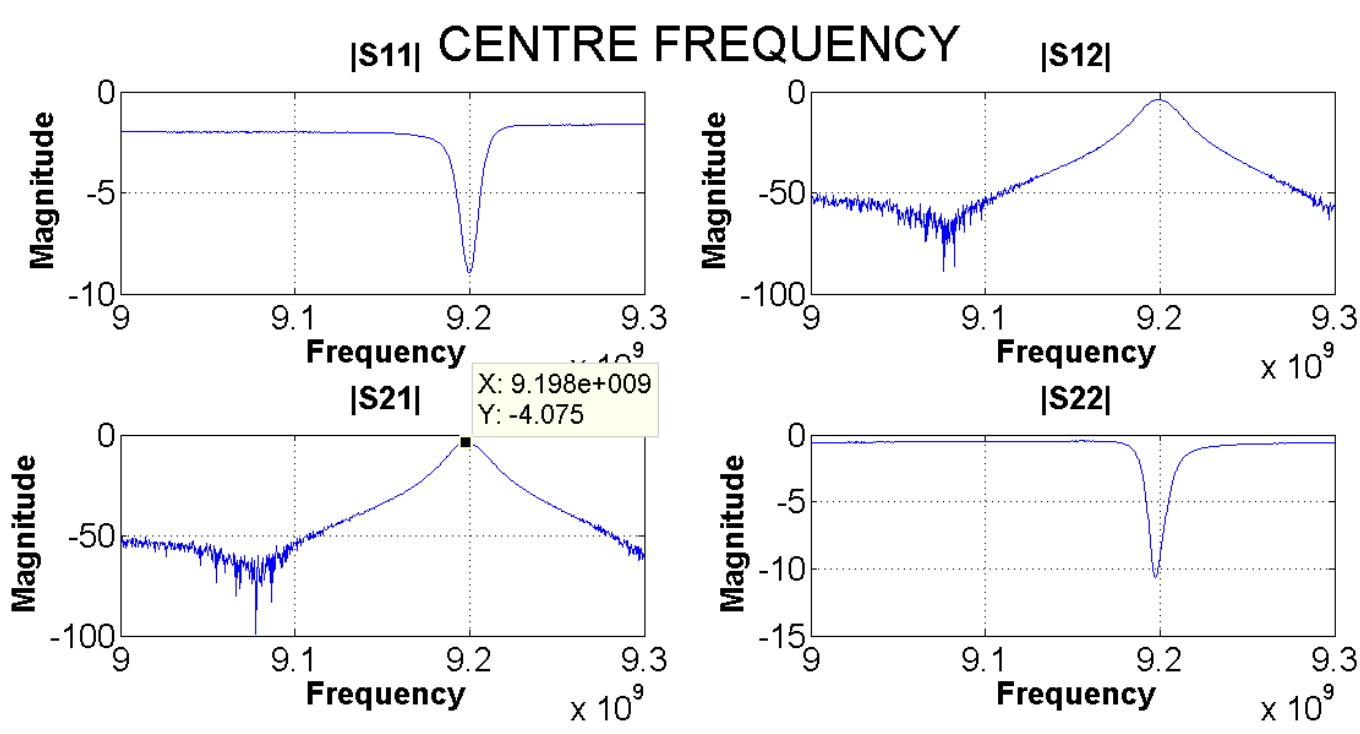

Figure 4.13: Measured S-parameters of filter tuned to the centre frequency. 

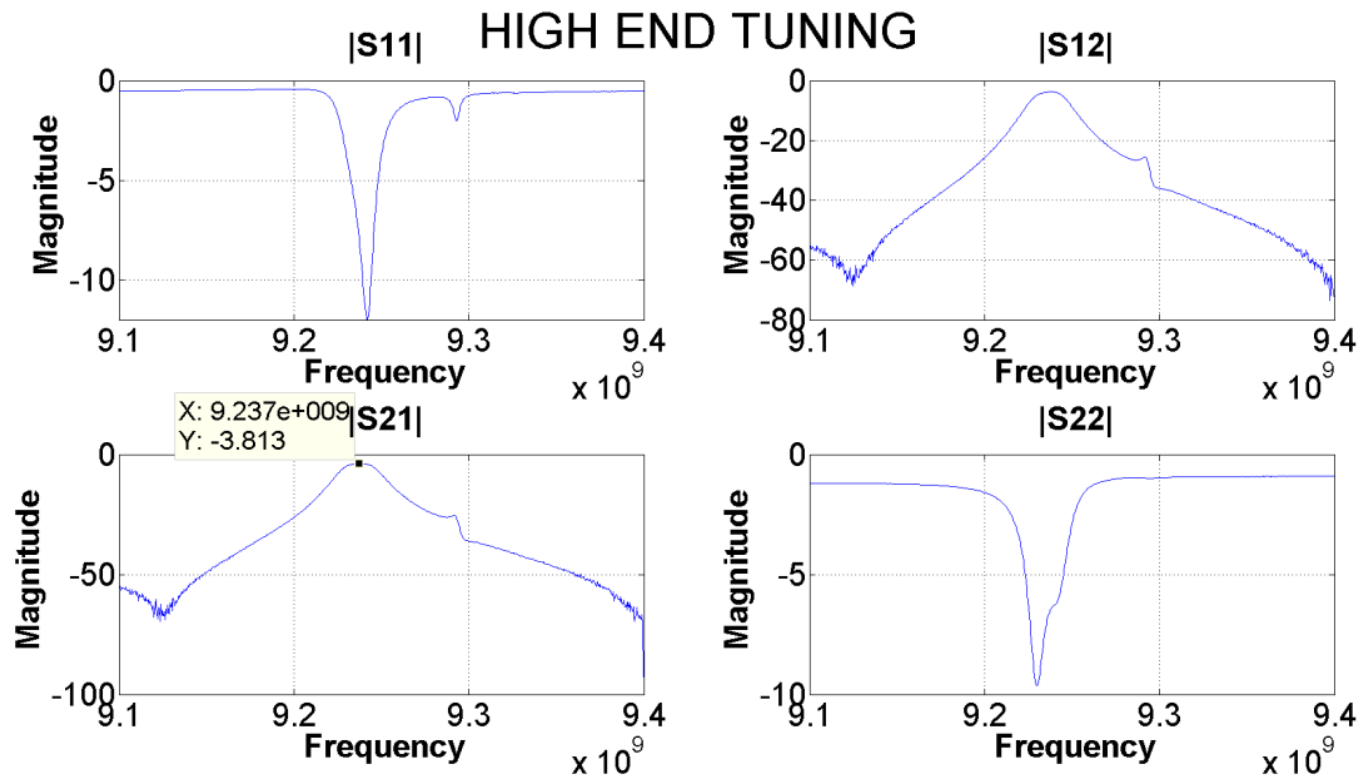

Figure 4.14: Measured S-parameters of filter tuned to its highest operating frequency.
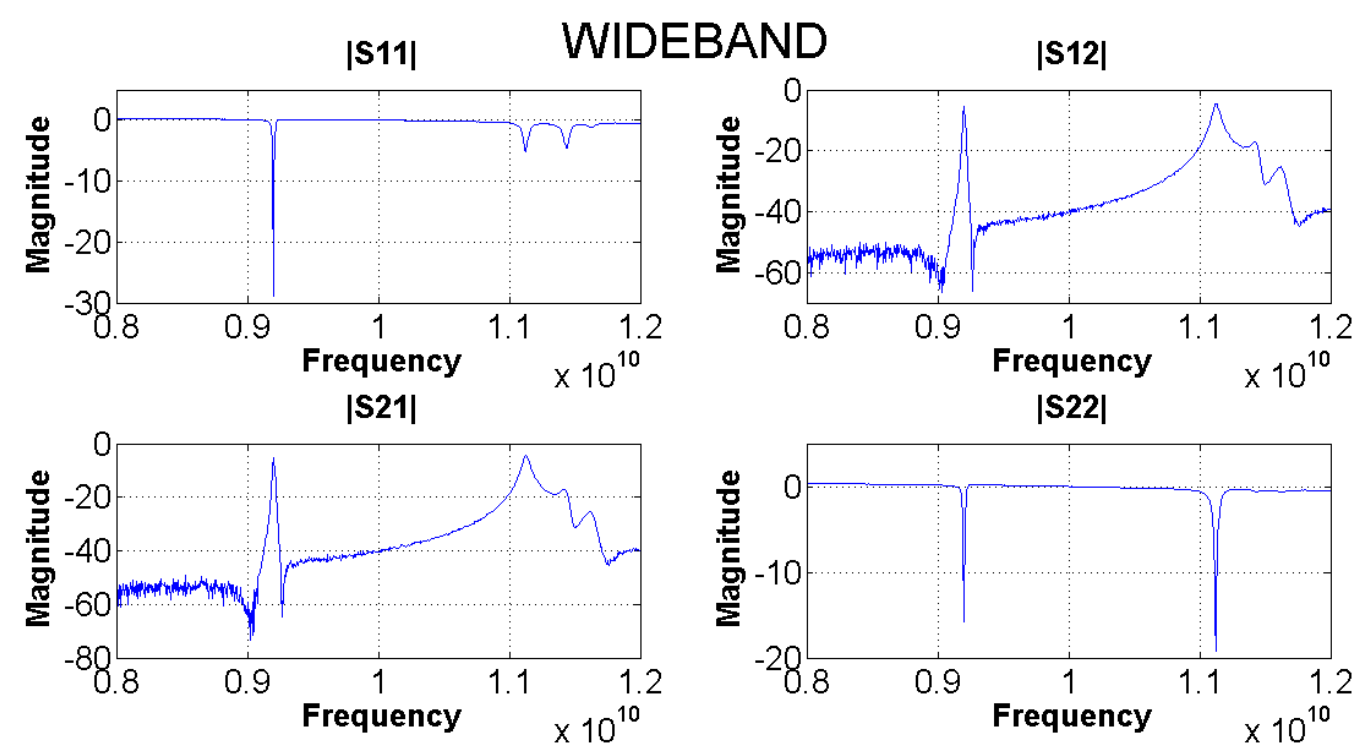

Figure 4.15: Wideband measurement of filter tuned to its centre frequency.

When tuning the filter, there is always a compromise between insertion loss and bandwidth. To ensure that the filter has a fairly narrow band with an acceptable insertion loss, an insertion loss of 4 $\mathrm{dB}$ was chosen which results in a bandwidth of approximately $15 \mathrm{MHz}$. Table 4-4 lists the insertion loss and bandwidth of the filter at the different frequencies.

Table 4-4: Insertion loss and bandwidth of filter tuned to the lowest, highest and centre frequency.

\begin{tabular}{|l|l|l|l|}
\hline & Frequency $\mathbf{( G H z )}$ & Insertion loss (dB) & Bandwidth (MHz) \\
\hline Low end & 9.06 & 4.121 & 12 \\
\hline Centre & 9.198 & 4.075 & 15 \\
\hline High end & 9.237 & 3.813 & 21 \\
\hline
\end{tabular}


It is clear from Table 4-4 that a lowering in insertion loss results in a wider bandwidth.

\subsection{Oscillator}

The tuning range of the oscillator is limited by the combined tuning ranges of the filter and resonator. The lower end tuning range is limited by the resonator at approximately $9.171 \mathrm{GHz}$, whereas the high end tuning range is limited by the filter at approximately $9.237 \mathrm{GHz}$ (state approximately as the VNA has a minimum step of $2 \mathrm{MHz}$, which is too wide. The frequency sweep mode is used which causes the precise frequency to be incorrect). The system can thus be tuned over a bandwidth of approximately $66 \mathrm{MHz}$. The oscillator used to obtain the final results is shown in Figure 4.16.

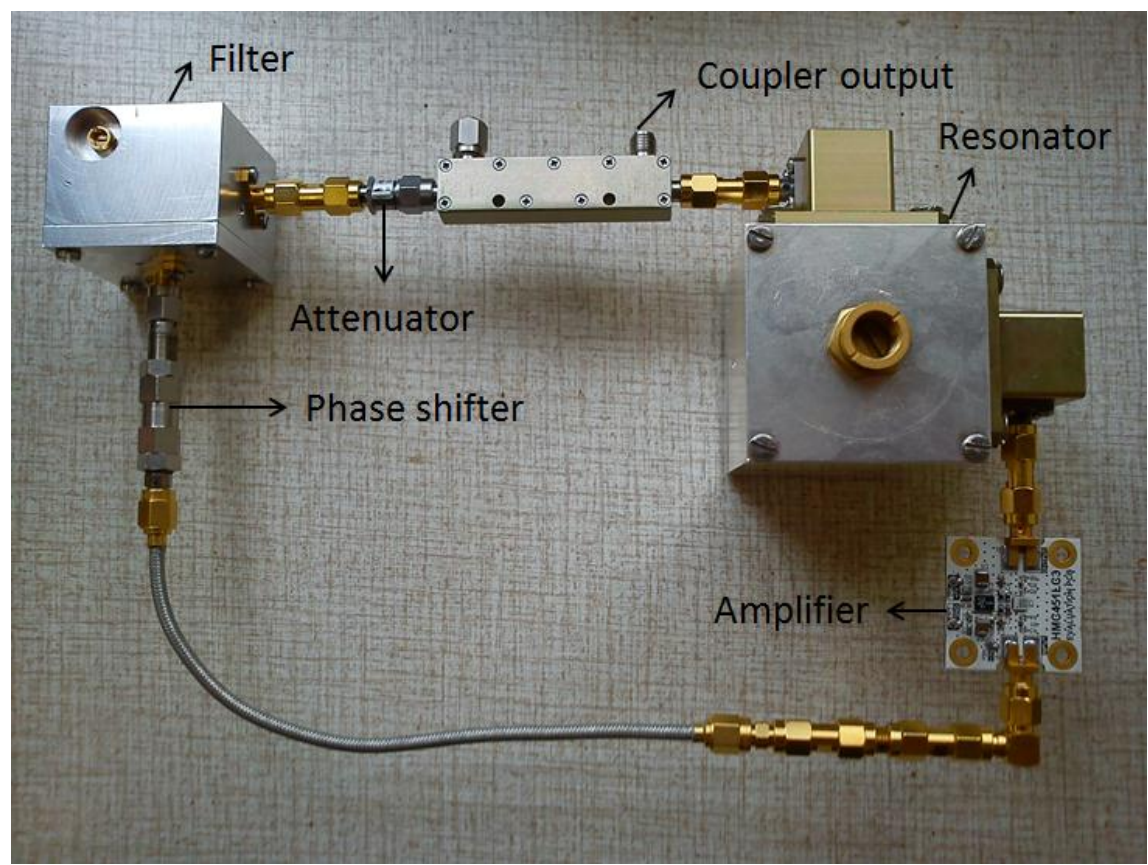

Figure 4.16: Waveguide cavity oscillator.

\subsubsection{Single oscillator measurements}

The final step is to cascade all the components forming the oscillator, and finally closing the loop. Rhea [3] states that the power can be coupled out anywhere in the loop, but better harmonic performance is obtained when coupling out directly after the frequency selective device.

In order to rise above the thermal noise floor of the measuring equipment, the output power must be maximised. This is done by taking the output directly after the amplifier. $A 3 \mathrm{~dB}$ attenuation pad is connected between the resonator and the filter in order to avoid their modes coupling to each other. The HMC564LC4 evaluation kit from Hittite is used as the active device and a $6 \mathrm{~dB}$ coupler is used to couple the power out. The datasheet of the active device is available online at http://www.hittite.com/content/documents/data sheet/hmc564lc4.pdf. A mechanical phase shifter is used to ensure a phase shift of zero degrees around the loop and to ensure operation at maximum phase slope of the resonator. 


\subsubsection{VNA measurement}

The oscillator is first measured on the VNA in an open-loop configuration to ensure operation at the maximum phase slope of the resonator. Figure 4.17 shows a schematic of this measurement configuration and Figure 4.18 shows a picture of the actual measurement setup.

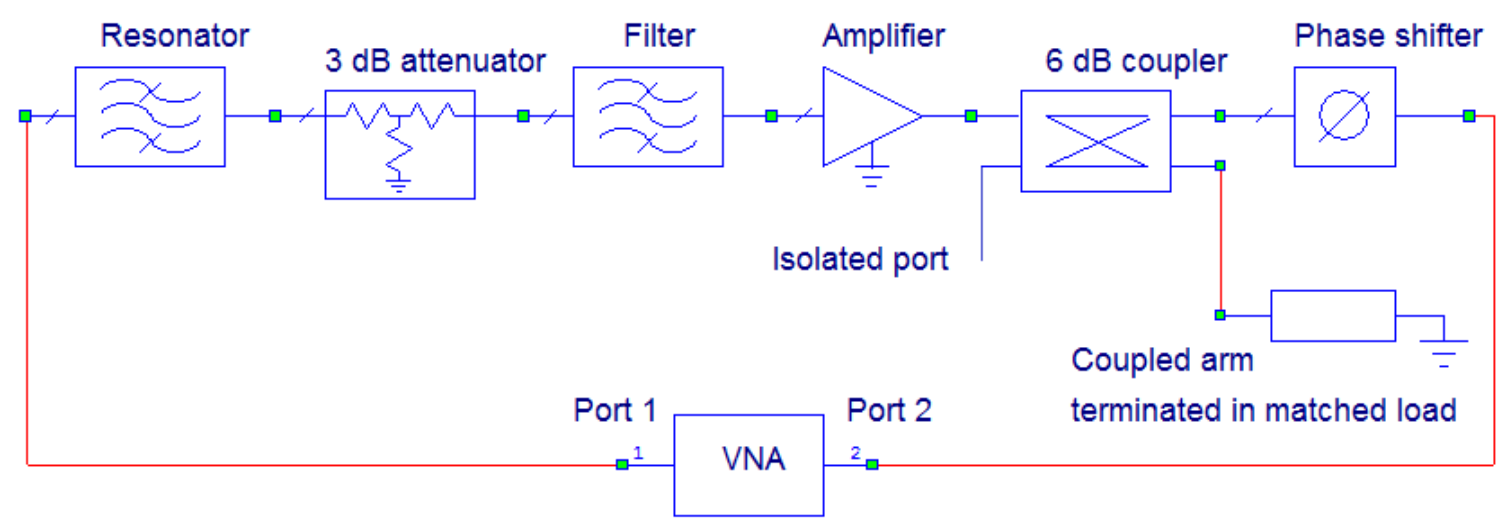

Figure 4.17: Schematic of open-loop measurement configuration.

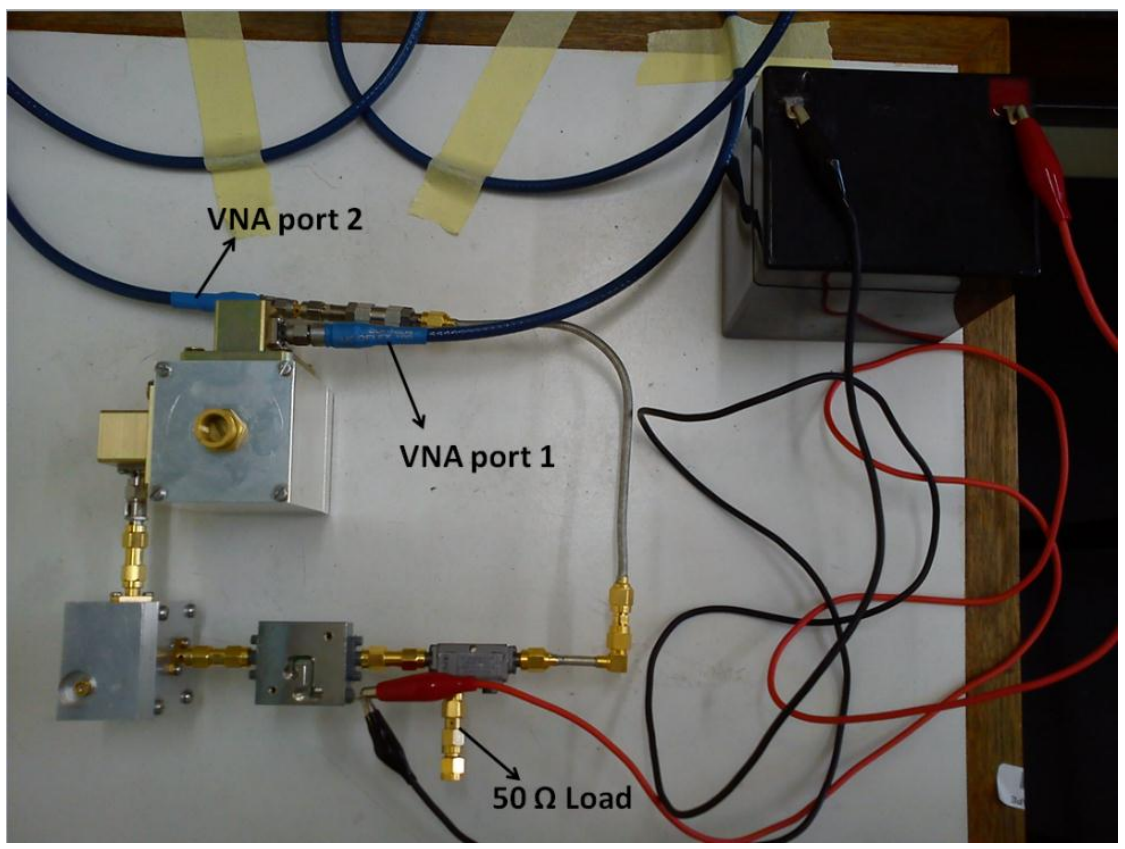

Figure 4.18: Open-loop measurement setup.

In order to ensure oscillation, the phase around the loop must be exactly zero degrees where the gain is larger than one. Using the phase shifter, the phase can be tuned to zero degrees at the frequency where the loop has maximum gain. In the case of the resonator designed in this thesis, this is also the point where the group delay is at a maximum and optimal performance is achieved. Figure 4.19 shows a measurement of the magnitude and phase of $S_{21}$ plotted together, obtained from the VNA. It is important to note that the coupled arm of the coupler must be terminated with a $50 \Omega$ load while performing these measurements. 


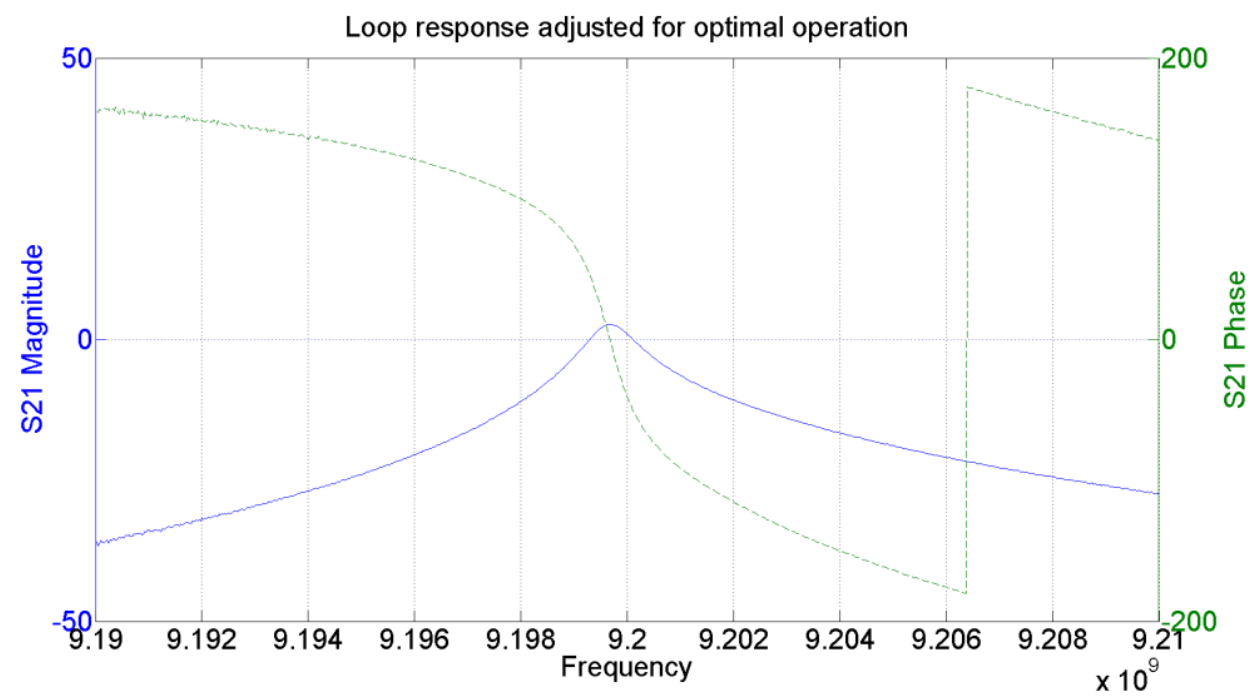

Figure 4.19: VNA measurement of open-loop oscillator tuned to operate at its centre frequency. The phase around the loop is tuned to exhibit a zero degree shift at its maximum slope, while the magnitude is larger than unity.

It is clear from the figure that the phase around the loop exhibits a zero degrees shift where the phase slope and the gain are at their maximum. It is desirable to operate the oscillator with a small gain margin in order for the oscillator not to be too non-linear. The loop has a gain margin of approximately $2.7 \mathrm{~dB}$ and is acceptable. Closing the loop will result in a signal oscillating at just below $9.2 \mathrm{GHz}$.

\subsubsection{Spectrum analyser measurement}

The loop is closed and the oscillator is tested on the R\&S FSEK3O spectrum analyser in order to confirm oscillation at the designed frequency and to obtain the output power. Figure 4.20 shows the output of the spectrum analyser and confirms oscillation at $9.2 \mathrm{GHz}$ with a power level of $6 \mathrm{dBm}$. 


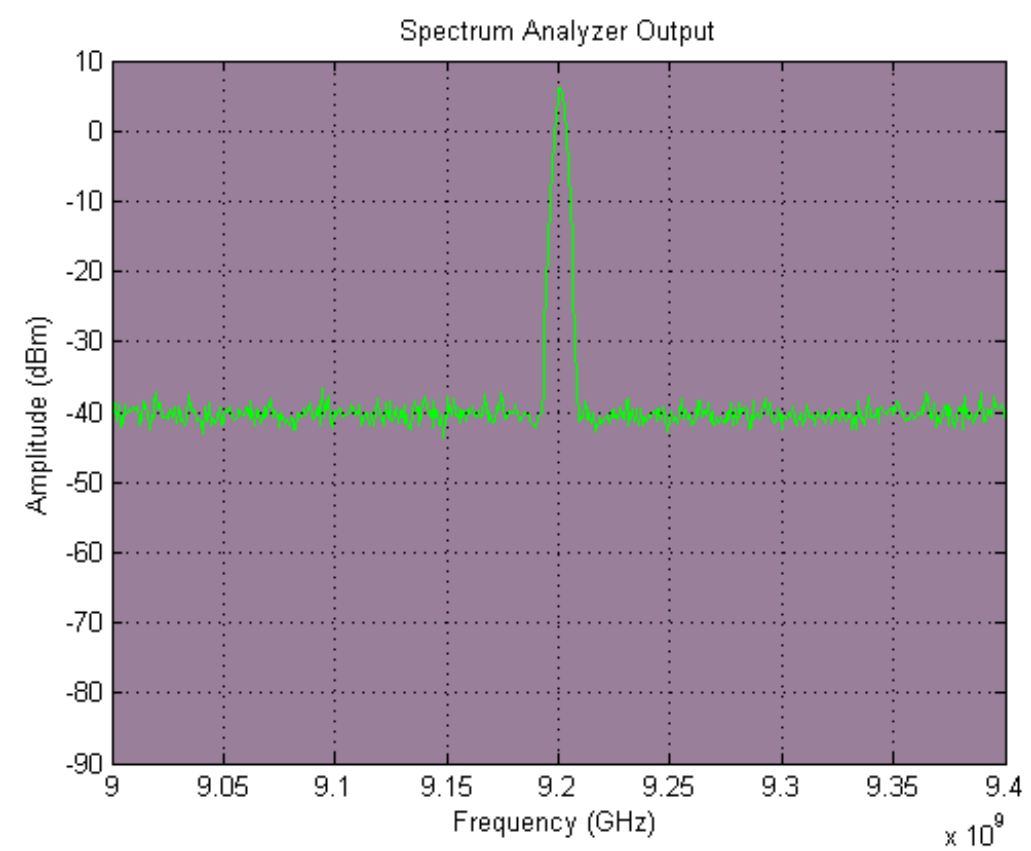

Figure 4.20: Output of oscillator indicating oscillation at $9.2 \mathrm{GHz}$ with a power level of $6 \mathrm{dBm}$.

The filter is removed from the oscillator in order to confirm its validity. The phase is again tuned with help of the VNA to meet the Barkhausen criteria and the loop is closed. Figure 4.21 shows the result obtained from the spectrum analyser.

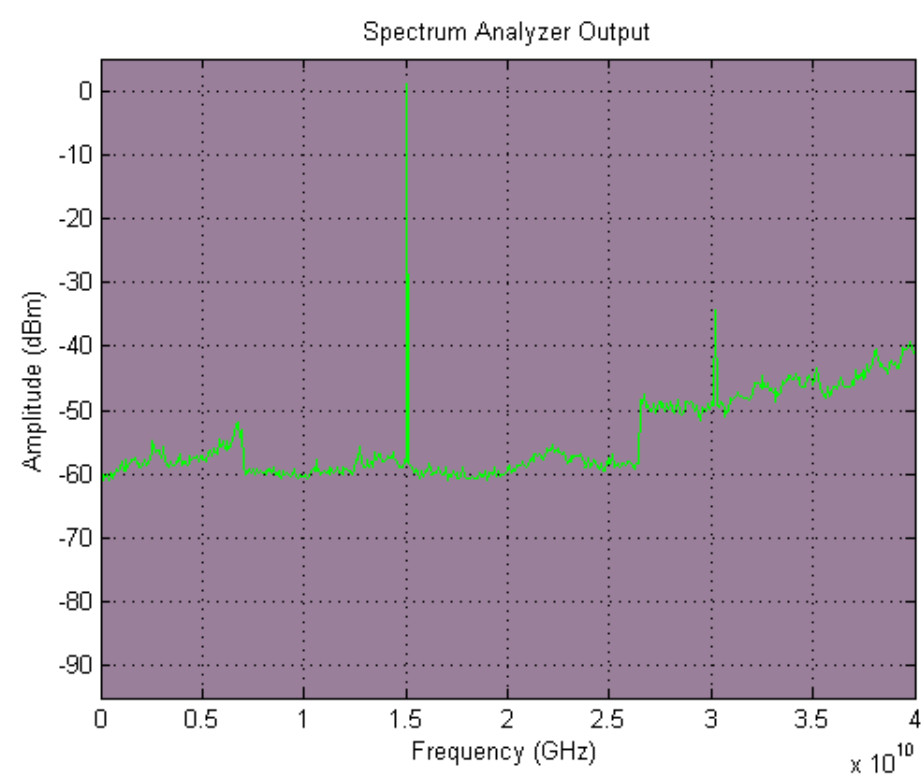

Figure 4.21: Spectrum of oscillator output when the filter is removed from the loop.

It is clear from the figure that the oscillator does not oscillate at the design frequency of $9.2 \mathrm{GHz}$, validating the use of the filter. The filter is reinserted into the oscillator loop and all the remaining measurements are done with all the necessary components present in the oscillator. 


\subsubsection{Phase noise measurement}

The phase noise of the oscillator is measured on the R\&S FSUP-B60 signal source analyser. The amplifier is biased with a battery in order to minimise noise. The Leeson frequency predicts a corner frequency at

$$
\begin{aligned}
f_{L} & =\frac{v_{0}}{2 Q_{l}} \\
& =400 \mathrm{kHz}
\end{aligned}
$$

Figure 4.22 shows the phase noise output of the oscillator obtained from the signal source analyser.

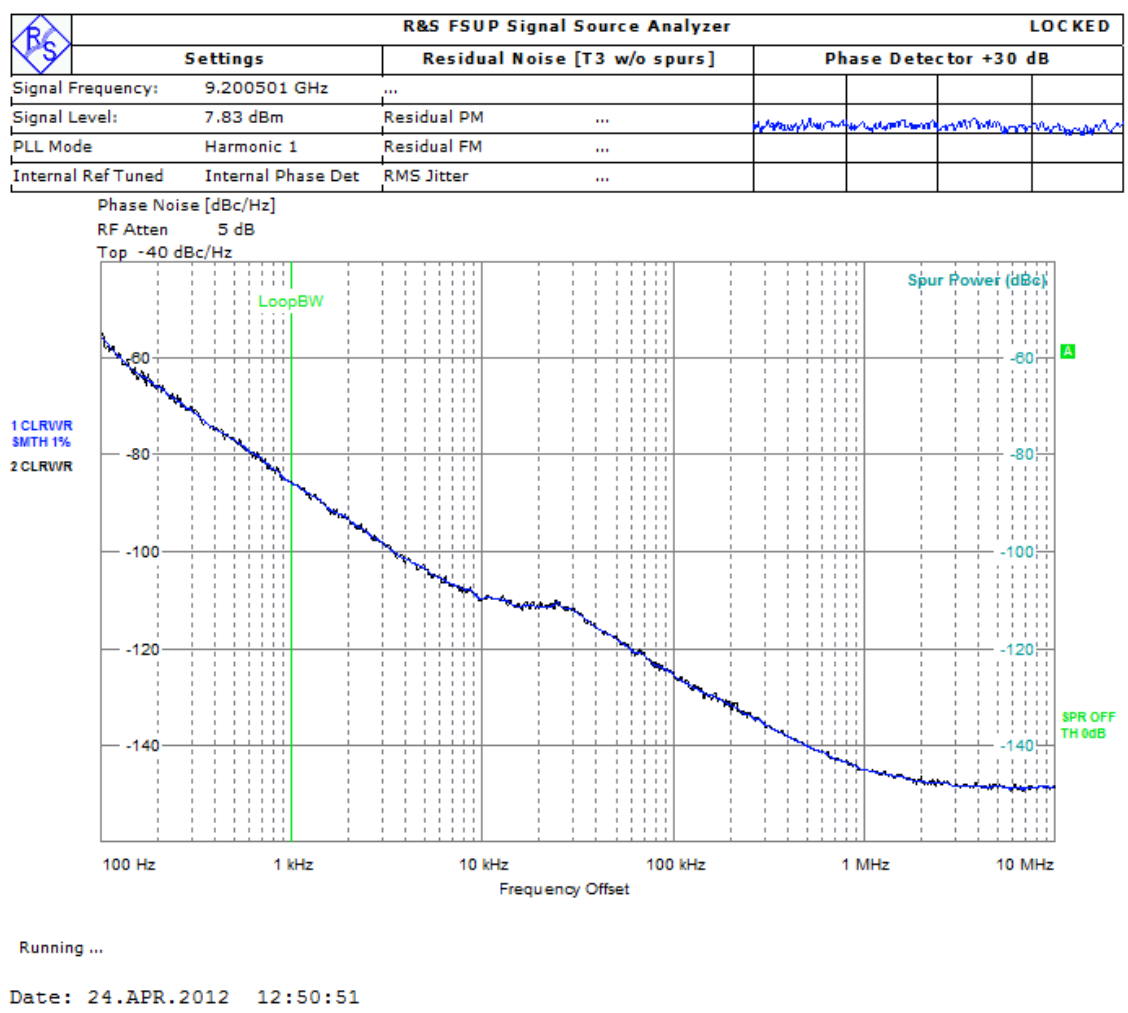

Figure 4.22: Measured phase noise of oscillator.

Inspecting this graph, it can be seen that the phase noise exhibits a constant value between $10 \mathrm{kHz}$ and $30 \mathrm{kHz}$ offset from the carrier. The documentation of the phase noise analyser reveals that the oscillator's phase noise has reached the phase noise sensitivity of the measuring instrumentation, which could explain this flat slope. The Leeson frequency also predicts a corner frequency at $\mathbf{4 0 0}$ $\mathrm{kHz}$, which is not present in this measurement. This phase noise measurement is invalid.

\subsubsection{Two-oscillator measurements}

Another procedure for measuring the phase noise of the oscillator must be implemented. One method is to use two identical oscillators oscillating at slightly different frequencies and downconverting the signals to an intermediate frequency, where the signal noise analyser is more sensitive. If the two oscillators were different, the resulting phase noise will be that of the oscillator with the poorest phase noise performance. It is thus necessary to manufacture an exact copy of the 
oscillator to obtain the same phase noise curves, at slightly different frequencies. One signal will serve as the LO signal and the other as the RF signal. The LO and RF signals can be represented as [6]

$$
\begin{aligned}
& v_{L O}(t)=\cos 2 \pi f_{L O} t \\
& v_{R F}(t)=\cos 2 \pi f_{R F} t
\end{aligned}
$$

Mixing these two signals results in a down-conversion and the IF signal is obtained as

$$
v_{I F}(t)=\frac{K}{2}\left[\cos 2 \pi\left(f_{R F}-f_{L O}\right) t+\cos 2 \pi\left(f_{R F}+f_{L O}\right) t\right]
$$

where $\mathrm{K}$ is a constant accounting for the voltage conversion loss of the mixer [6]. Figure 4.23 and Figure 4.24 illustrates this process.

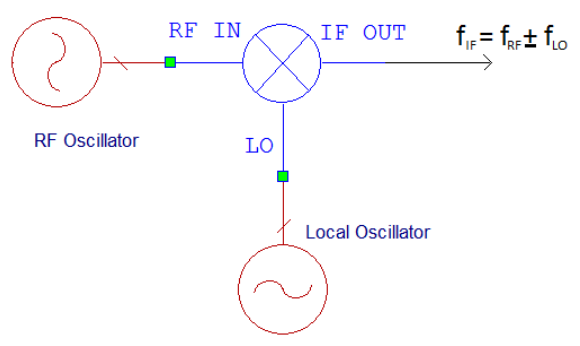

Figure 4.23: Frequency down-conversion using a mixer.

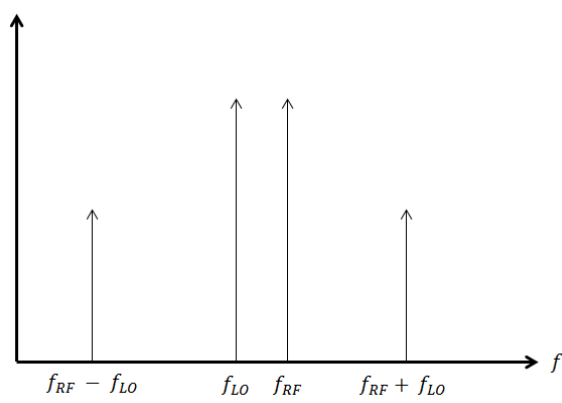

Figure 4.24: Frequency spectrum of down-conversion process.

An exact copy of the resonator and the filter were manufactured and two amplifiers and couplers of the same type were used in the two oscillator circuits. As stated at the beginning of this section, the oscillator can be tuned from approximately $9.171 \mathrm{GHz}$ to $9.237 \mathrm{GHz}$. One oscillator is tuned to the high end and serves as the RF signal, while the other one is tuned to the low end and serves as the LO signal to the mixer. Due to the fact that the Q-factor of the resonator used in this oscillator is barely changed by tuning it over a small frequency range, the two oscillators' performances will be essentially the same at the high and low end. The amplifiers used in the following measurements are the HMC451LC3 from Hittite and its datasheet is available online at http://www.hittite.com/content/documents/data sheet/hmc451lc3.pdf. The amplifier has a gain of $19 \mathrm{~dB}$ with a noise figure of $7 \mathrm{~dB}$ and the output power for $1 \mathrm{~dB}$ compression ( $\mathrm{P} 1 \mathrm{~dB}$ ) is $19.5 \mathrm{dBm}$. The 
amplifier is biased via a low noise voltage regulator, LTC 3029. Identical $10 \mathrm{~dB}$ couplers are used in the oscillators. The use of different amplifiers and couplers in this section, compared to section 4.3.1, is due to the availability of the components. The mixer used for the down-conversion process is the HMC52OLC4 from Hittite and its datasheet is available online at http://www.hittite.com/content/documents/data sheet/hmc520lc4.pdf.

The phase noise performance of two different oscillator configurations will be investigated. In the first configuration, the power is coupled out directly after the amplifier while in the second configuration the power is coupled out directly after the resonator.

\subsubsection{Configuration 1}

The two oscillators are tuned to their respective operating frequencies while ensuring operation at the maximum phase slope of the resonator. The phase noise is first measured in configuration 1. In order to confirm that the two oscillators are identical, the spectrum analyser measurements are also included. A schematic of the oscillator configuration considered in this section is shown in Figure

4.25 .

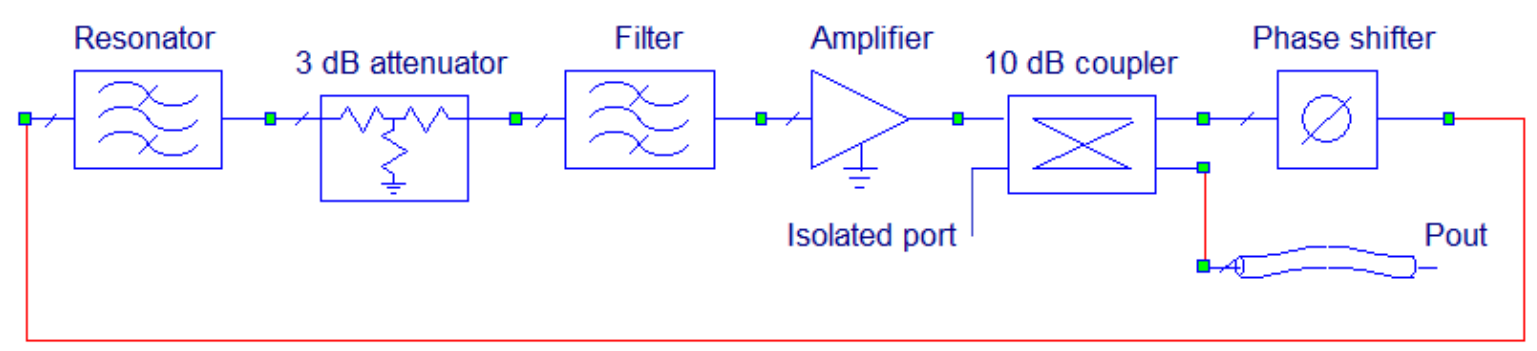

Figure 4.25: Configuration 1: Oscillator configuration with the output taken directly after the amplifier.

\subsection{Spectrum analyser measurements}

The spectra of the two oscillators are shown in Figure 4.26 and Figure 4.27. It is clear that the output power level of the LO oscillator is higher than the RF oscillator. This phenomenon might be explained by arguing that the amplifier used in the RF oscillator has a lower compression point compared to the amplifier in the LO oscillator due to tolerances in manufacturing. Another explanation might suggest that the oscillators were not tuned to operate exactly in the high slope region of the resonator which could lead to loss of output power. It should be noted that it is difficult to tune the oscillators to operate in the high slope region of the resonator. This is partly due to the use of mechanical phase shifters which is difficult to tune precisely. Also, by the closing the loop some of the cables might bend slightly, which will influence the phase around the loop. 


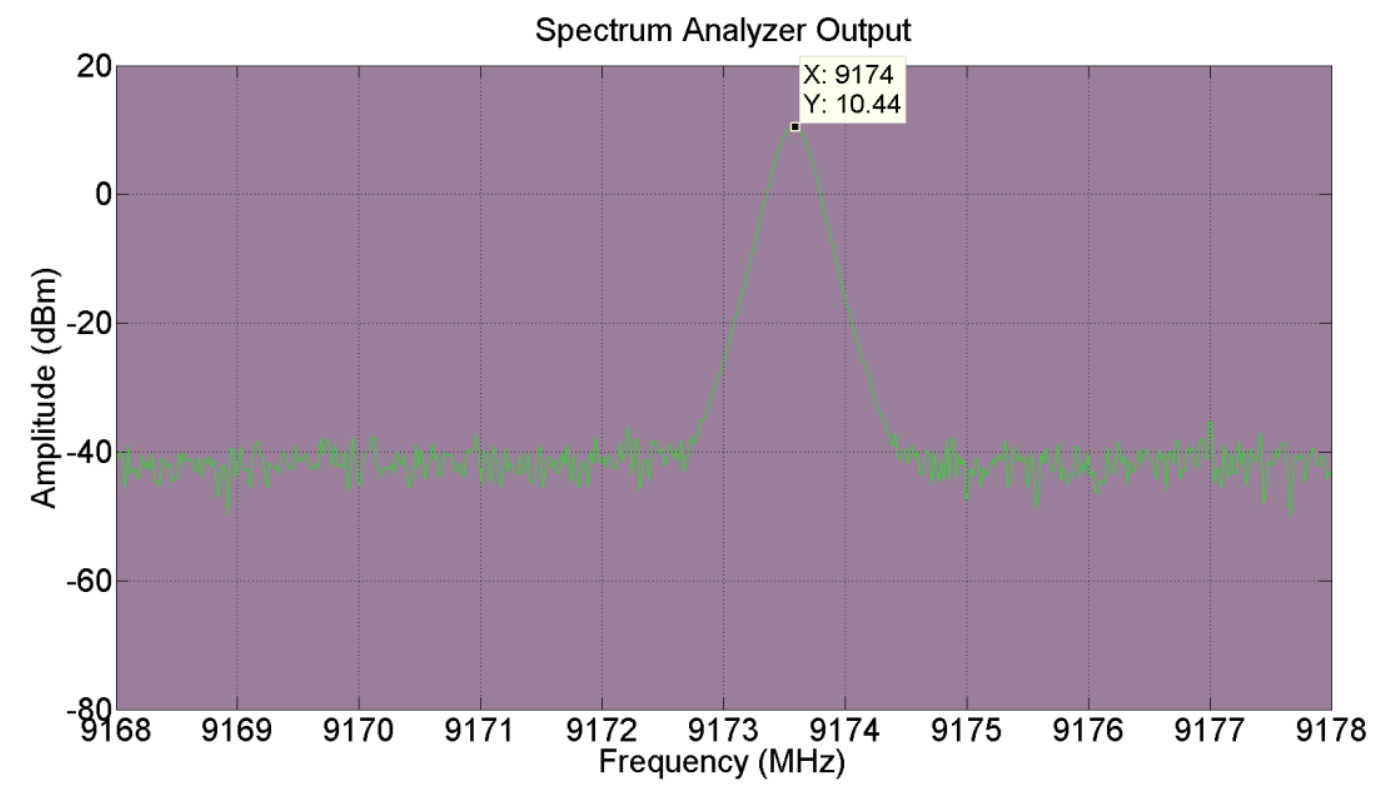

Figure 4.26: Close-in spectrum of LO oscillator output.

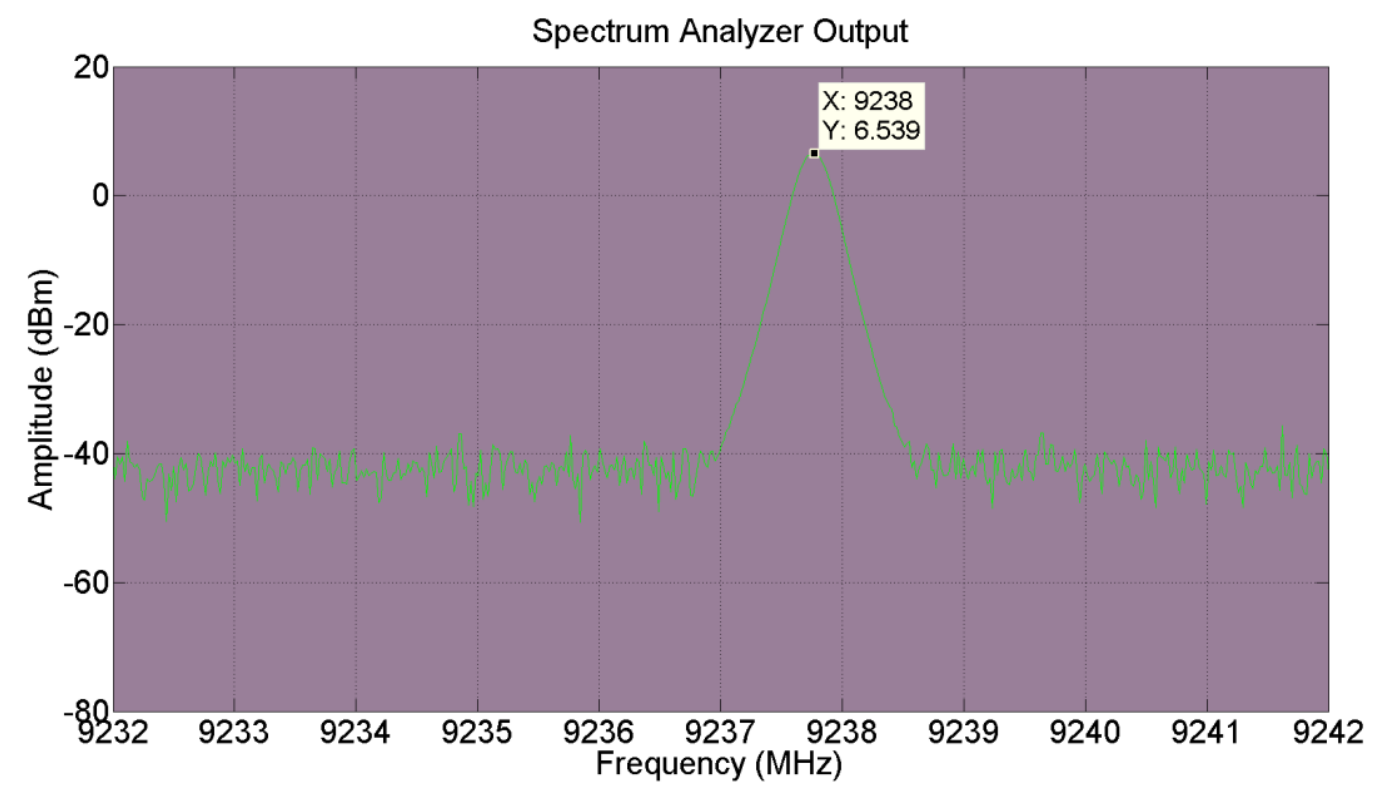

Figure 4.27: Close-in spectrum of RF oscillator output.

\subsection{Phase noise measurements}

Figure 4.28 and Figure 4.29 shows the individual phase noise measurements of the oscillators serving as the LO and RF signals to the mixer, respectively. 
CHAPTER 4

MEASUREMENTS

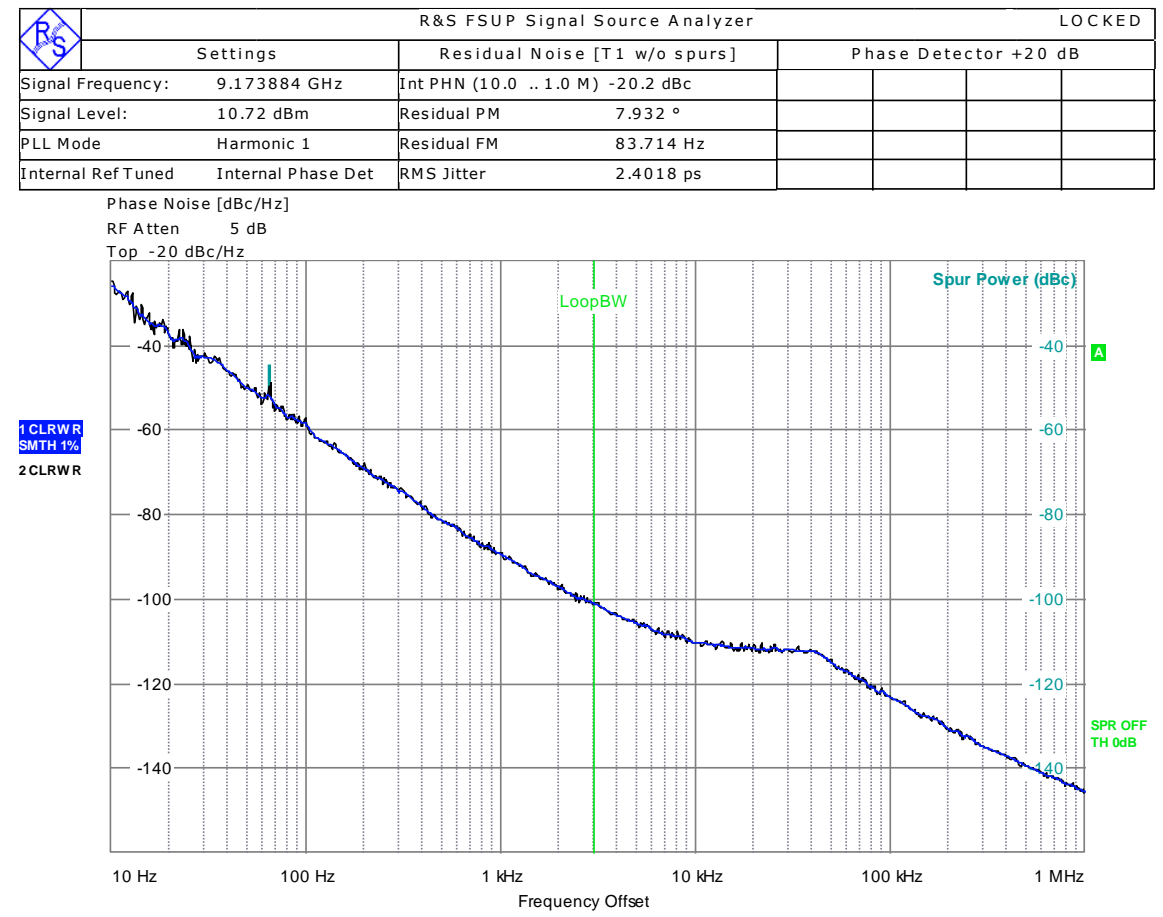

Running ...

Date: 7.NOV.2012 14:07:02

Figure 4.28: Phase noise measurement of LO oscillator.

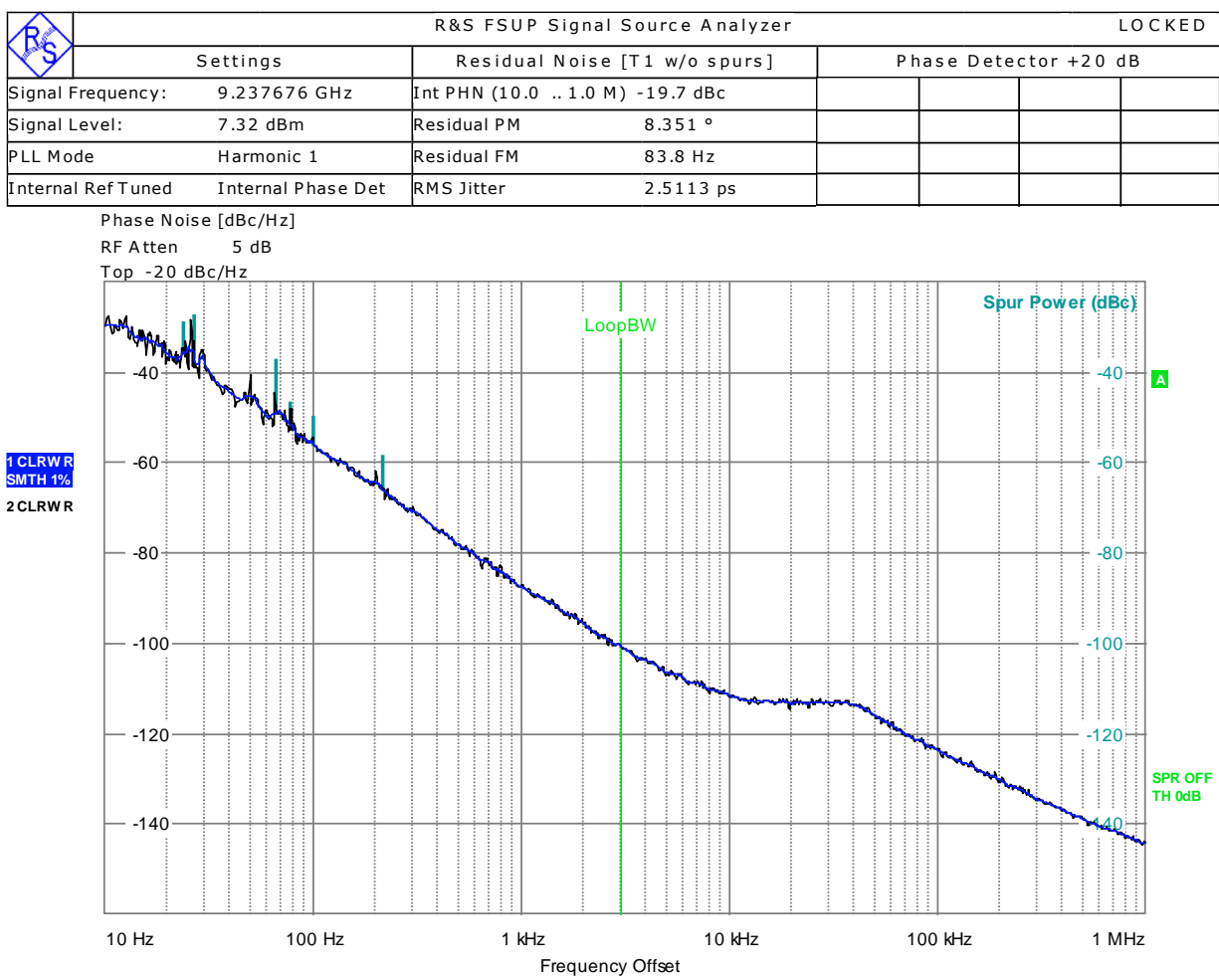

Running ...

Date: 7.NOV.2012 13:50:22

Figure 4.29: Phase noise measurement of RF oscillator. 
It is clear that the two oscillators still possess the same phase noise curves as obtained in section 4.3.1.3. By down-converting these signals to an IF frequency, where the instrumentation is more sensitive, it would be possible to measure the phase noise of the oscillator in question. If the two oscillators had the same output power, a $3 \mathrm{~dB}$ correction had to be made to this phase noise result. This follows from the fact that if the phase noise of the two oscillators are the same, their phase noise are combined when the signals are mixed to the IF signal. In this case the two oscillators' output powers are not the same and the phase noise of one oscillator will dominate. Figure 4.30 shows the resulting phase noise curve.

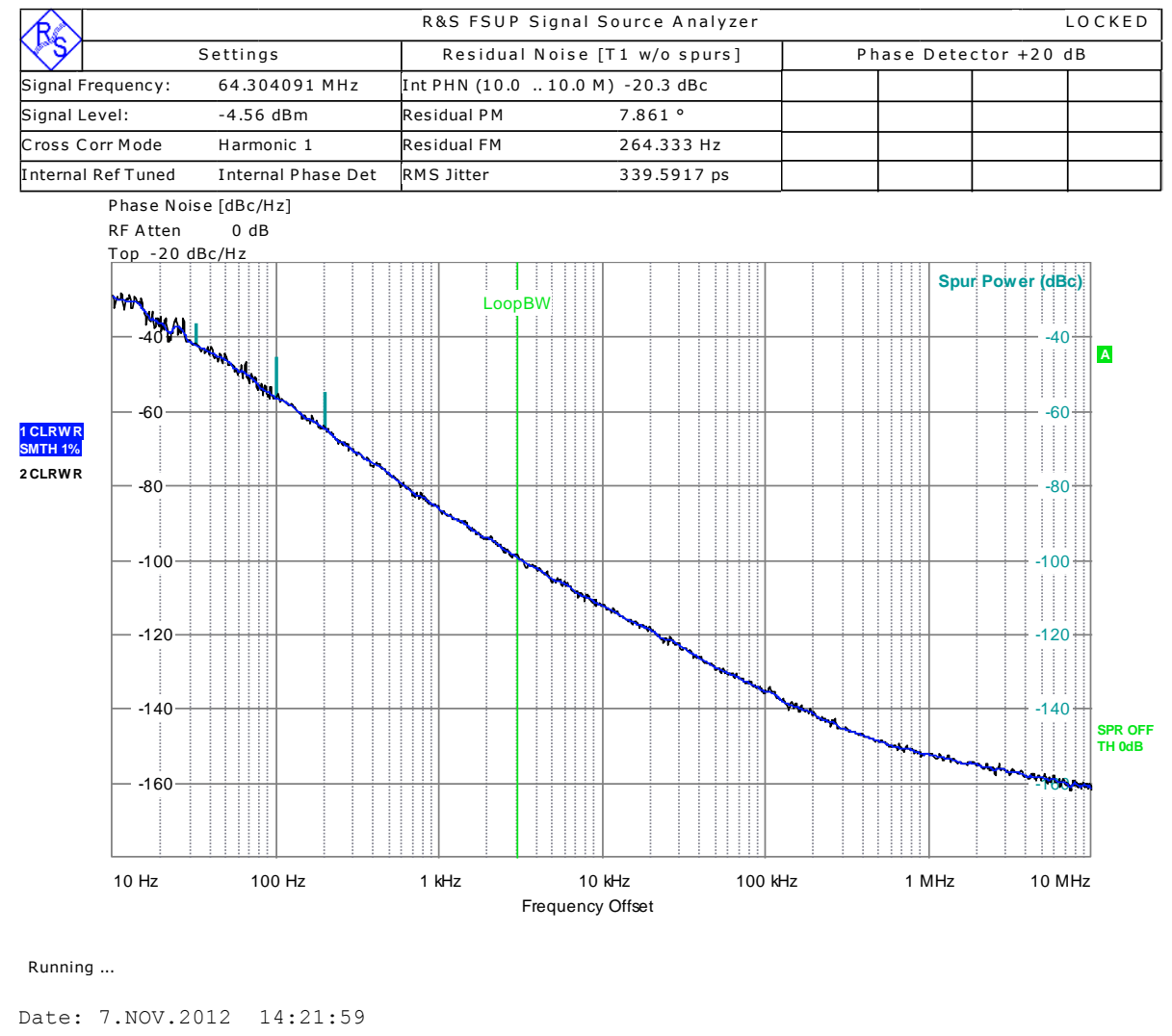

Figure 4.30: Phase noise measurement of down-converted RF signal.

The corner frequency predicted by Leeson's frequency ( $400 \mathrm{kHz}$ ) can now clearly be seen from this plot. The slope of the curve is $-27.5 \mathrm{~dB} /$ decade from $10 \mathrm{~Hz}$ up to approximately $10 \mathrm{KHz}$ offset. Between $10 \mathrm{KHz}$ and $400 \mathrm{KHz}$ offset, the slope is $-23 \mathrm{~dB} /$ decade and from $400 \mathrm{KHz}$ to $10 \mathrm{MHz}$ offset, the slope is $-10 \mathrm{~dB} /$ decade. From $10 \mathrm{MHz}$ offset and onwards, the slope of the curve is flat with a noise power level of $-160 \mathrm{dBc} / \mathrm{Hz}$.

Leeson predicts a slope of $-30 \mathrm{~dB} /$ decade from $0 \mathrm{~Hz}$ offset to $\mathrm{f}_{\mathrm{L}}(400 \mathrm{kHz})$, followed by a slope of -10 $\mathrm{dB} /$ decade until $\mathrm{f}_{\mathrm{c}}$ (the corner frequency of the amplifier) and a flat slope from $\mathrm{f}_{\mathrm{c}}$ onwards for $\mathrm{a}$ high- $Q$ resonator case. The measured phase noise fits very well into Leeson's prediction of oscillator phase noise.

The SSB phase noise power is listed in Table 4-5 at various offset frequencies. 
Table 4-5: SSB phase noise power at various offset frequencies.

\begin{tabular}{|l|l|}
\hline Offset frequency $[\mathrm{Hz}]$ & $\mathbf{d B c} / \mathrm{Hz}$ \\
\hline 10 & -29.2 \\
\hline 100 & -57 \\
\hline $1 \mathrm{k}$ & -86.2 \\
\hline $10 \mathrm{k}$ & -111.5 \\
\hline $100 \mathrm{k}$ & -135.4 \\
\hline $1 \mathrm{M}$ & -151.5 \\
\hline $10 \mathrm{M}$ & -160 \\
\hline
\end{tabular}

\subsubsection{Configuration 2}

The oscillator's phase noise performance is investigated with the configuration shown in Figure 4.31. It differs from configuration 1 in that the output is taken directly after the resonator and the amplifier is placed directly before the resonator (as opposed to before the phase shifter and other cables) in order to maximise the output power. Measurements using the spectrum analyser are included again.

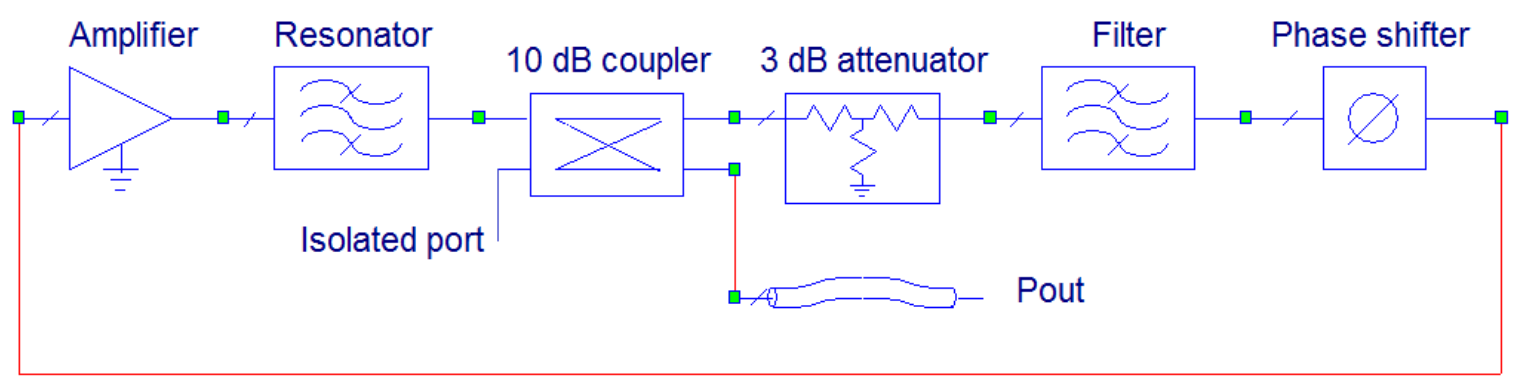

Figure 4.31: Configuration 2: Oscillator configuration with the output taken directly after the resonator.

\subsection{Spectrum analyser measurements}

The oscillators are tuned to operate in the maximum phase slope regions of the resonators using the VNA. The loops are closed and the outputs are measured with the spectrum analyser. The output of the LO and RF oscillators are shown in Figure 4.32 and Figure 4.33, respectively. 


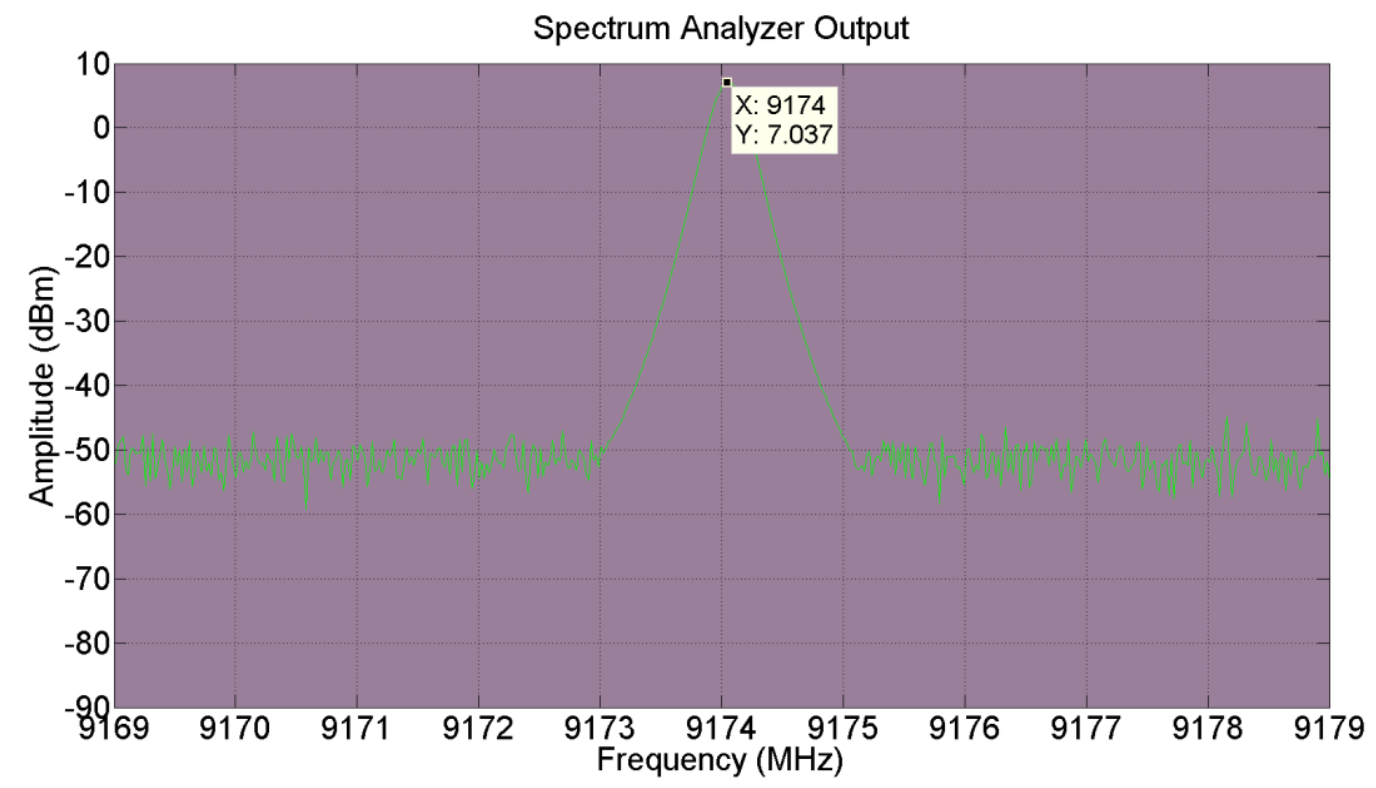

Figure 4.32: Close-in spectrum of LO oscillator output.

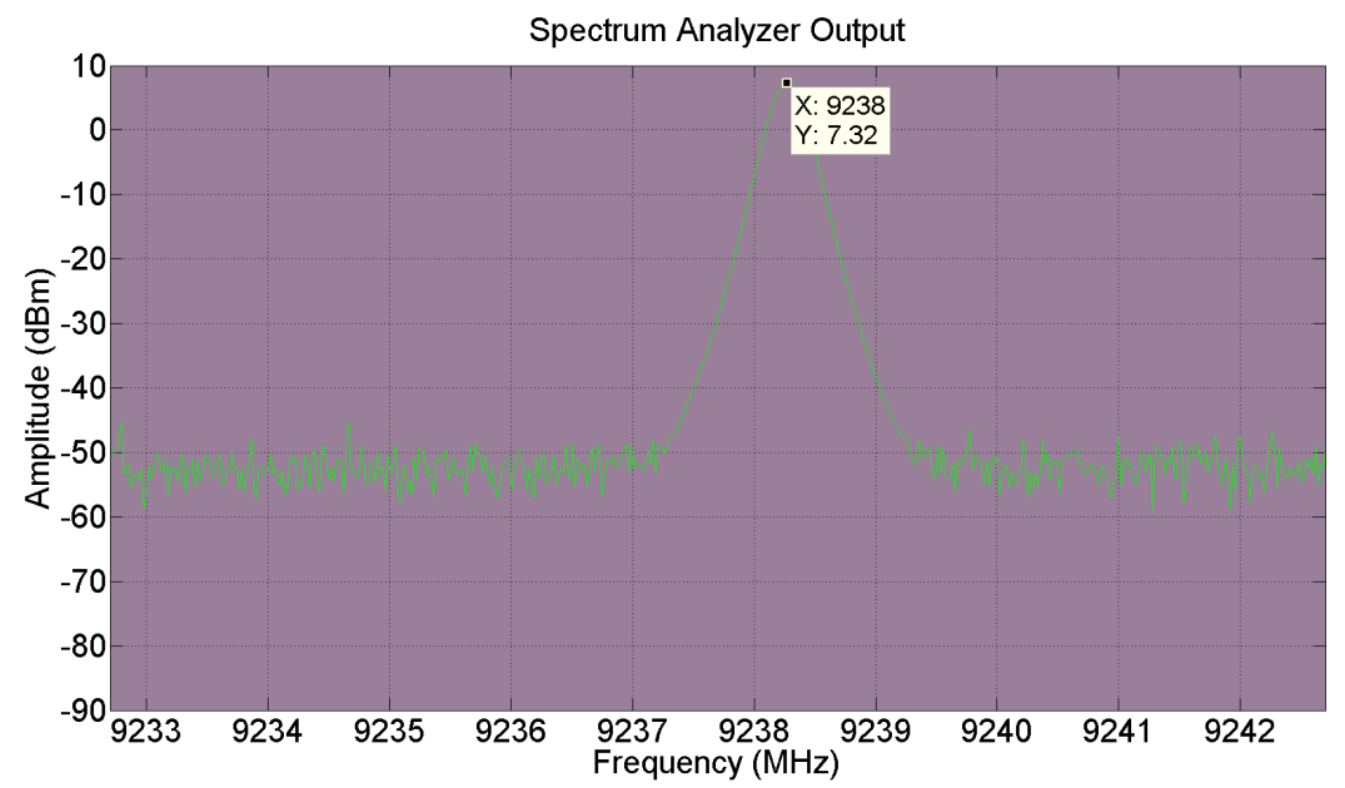

Figure 4.33: Close-in spectrum of RF oscillator output.

It is clear from these measurements that the output powers of these two oscillators are essentially the same. This concludes that their phase noise performance will essentially be identical. It follows that the phase noise of the two oscillators will be combined and a $3 \mathrm{~dB}$ correction must be made to the resulting phase noise curve.

\subsection{Phase noise measurements}

The output of the LO and RF frequency oscillators are taken to the LO and RF ports of the mixer, respectively. The resulting phase noise measurement is shown in Figure 4.34. 


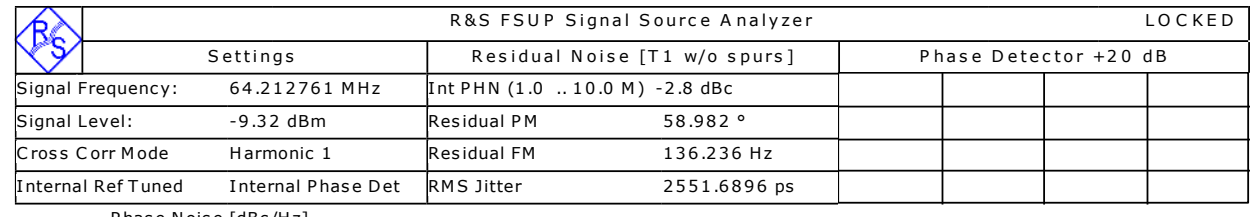
Phase Noise $[\mathrm{dBc} / \mathrm{Hz}]$

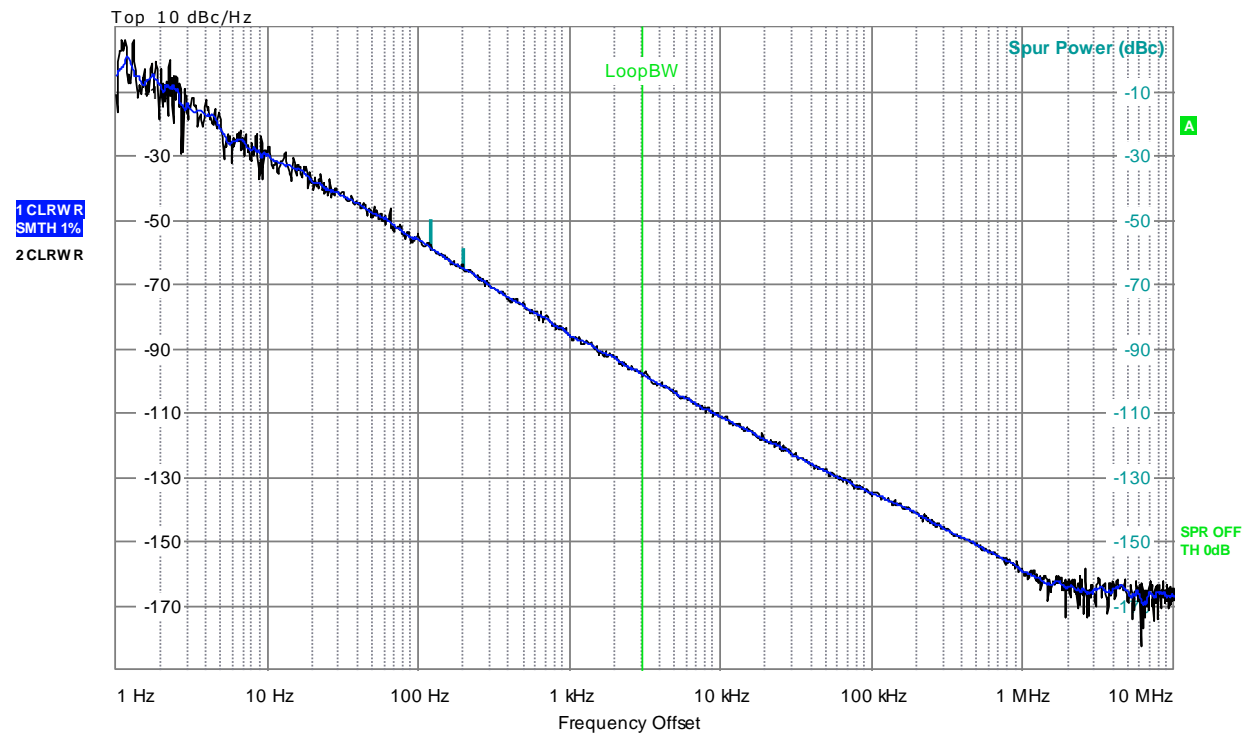

Measurement A borted

Date: 8.NOV.2012 10:45:49

Figure 4.34: Phase noise measurement of down-converted RF signal.

The advantage of taking the output directly after the resonator can be seen at offset frequencies greater than the Leeson frequency. Inspecting this plot, the Leeson frequency is no longer visible. This improvement in phase noise performance at higher frequencies can be explained by the additional filtering action of the resonator on the amplifier output. In other words, the $10 \mathrm{~dB} / \mathrm{decade}$ slope is suppressed by the filtering action of the resonator. The thermal noise floor of the amplifier is also suppressed by the resonator. With an input power to the measurement equipment of -9.32 $\mathrm{dBm}$ and noting that the thermal noise are equally due to amplitude noise and phase noise, the thermal phase noise floor of the system is calculated as

$$
\begin{aligned}
\mathcal{L}(f)_{\text {thermal }} & =(k T)_{d B m}-P_{\text {in }(d B m)}-3 \\
& =-174-(-9.32)-3 \\
& =-167.6 \mathrm{dBc} / \mathrm{Hz}
\end{aligned}
$$

This is in excellent agreement with the results from the plot. The phase noise powers at various offset frequencies, with the $3 \mathrm{~dB}$ correction, are listed in Table 4-6. 
Table 4-6: SSB phase noise power at various offset frequencies with the 3 dB correction.

\begin{tabular}{|l|l|}
\hline Offset frequency $[\mathrm{Hz}]$ & $\mathbf{d B c} / \mathbf{H z}$ \\
\hline 10 & -33 \\
\hline 100 & -59.6 \\
\hline $1 \mathrm{k}$ & -88.2 \\
\hline $10 \mathrm{k}$ & -114 \\
\hline $100 \mathrm{k}$ & -137.8 \\
\hline $1 \mathrm{M}$ & -161.6 \\
\hline $10 \mathrm{M}$ & -167 \\
\hline
\end{tabular}

\subsubsection{Measurements using a different active device}

One more phase noise measurement is performed with a different amplifier. The amplifier used in the following measurement is the DB01-0485 from Narda and its datasheet is attached in Appendix C. This is a low noise amplifier with a noise figure of $2.7 \mathrm{~dB}$, a gain of $18 \mathrm{~dB}$ and a $\mathrm{P} 1 \mathrm{~dB}$ of $15 \mathrm{dBm}$. The oscillators are set up in configuration 1. A $20 \mathrm{~dB}$ coupler and a $6 \mathrm{~dB}$ coupler were used in the RF and LO oscillators, respectively. The reason for these values of couplers is due to the availability of the components at the time. The phase noise performance of the down-converted signal using this amplifier is shown in Figure 4.35 and the phase noise levels at various offset frequencies are listed in Table 4-7.

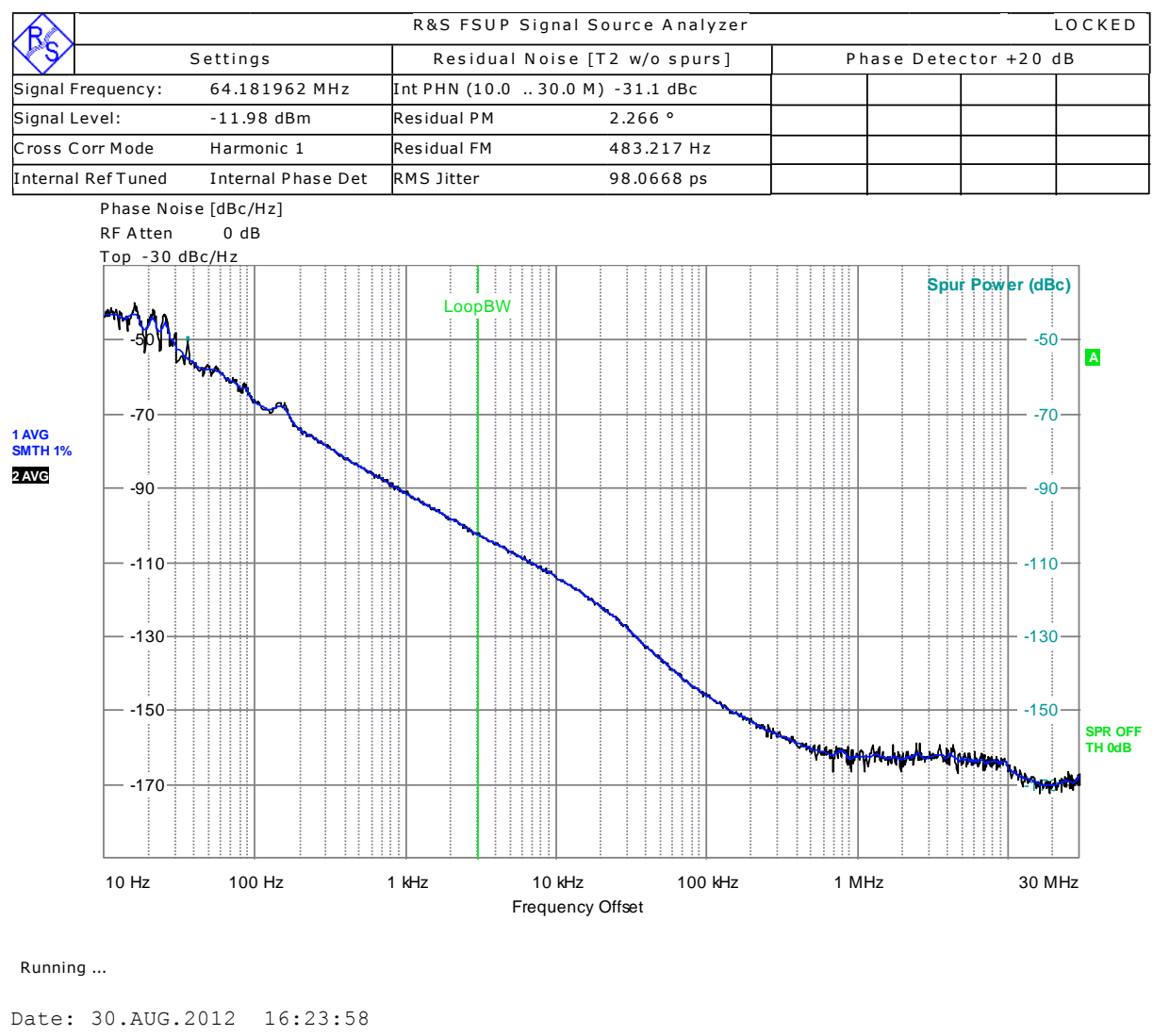

Figure 4.35: Phase noise measurement of down-converted signal of oscillators using DB01-0485 amplifiers as active devices. 
Table 4-7: SSB phase noise power of oscillator, with DB01-0485 amplifier as active device, at various offset frequencies.

\begin{tabular}{|l|l|}
\hline Offset frequency $[\mathrm{Hz}]$ & $\mathbf{d B c} / \mathbf{H z}$ \\
\hline 10 & -43 \\
\hline 100 & -66.9 \\
\hline $1 \mathrm{k}$ & -91.5 \\
\hline $10 \mathrm{k}$ & -114.8 \\
\hline $100 \mathrm{k}$ & -146.1 \\
\hline $1 \mathrm{M}$ & -162.3 \\
\hline $10 \mathrm{M}$ & -166.1 \\
\hline
\end{tabular}

The Leeson frequency is clearly visible at $400 \mathrm{kHz}$. The plot exhibits a dip at approximately $20 \mathrm{kHz}$. This dip might be explained by the absence of a low noise voltage regulator biasing the amplifier, resulting in an increase in phase noise due to the noise of the power supply. The phase noise plot exhibits a slope of approximately $-25 \mathrm{~dB} /$ decade between $10 \mathrm{~Hz}$ and $20 \mathrm{kHz}$ offset. Between $20 \mathrm{kHz}$ and $100 \mathrm{kHz}$, a slope of approximately $-35 \mathrm{~dB} /$ decade is observed after which the slope continues on at $-25 \mathrm{~dB} /$ decade until $500 \mathrm{kHz}$ where it reaches the thermal noise floor of the measuring system with a phase noise power level of $-162.3 \mathrm{dBc} / \mathrm{Hz}$. If the amplifier used in this measurement made use of a low noise voltage regulator, the noise from the power supply would be suppressed and better phase noise performance should be observed at offset frequencies less than $20 \mathrm{kHz}$.

\subsubsection{Comparison to commercially available oscillators and an ultra-low-noise STALO}

In this section, the phase noise performance of the oscillator designed in this thesis is compared to the phase noise performance of two commercially available low noise oscillators with very good performance, whose components are not cryogenically cooled and the phase noise performance of the ultra-low-noise STALO designed in [22]. The performance of the oscillator with the Hittite HMC451LC3 amplifier as the active device, which is set up in configuration 2, and the oscillator with the Narda DB01-0485 amplifier as active device, which is set up in configuration 1, are both used for comparison.

The two high performance low noise oscillators that will be used to compare to the oscillator designed in this thesis is the HMC-C200 from Hittite and the DRO-10.600-FR from Poseidon Scientific Instruments (PSI). Both of these oscillators are dielectric resonator oscillators (DRO) and their datasheets are presented in Appendix D and Appendix E. The phase noise performances of these oscillators are listed in Table 4-8, specified at their operating frequencies.

Table 4-8: Phase noise performances of oscillators that will be used to compare to the oscillator designed in this thesis.

\begin{tabular}{|l|l|l|l|}
\hline & \multicolumn{3}{|c|}{ Phase noise power [dBc/Hz] } \\
\hline Offset frequency [Hz] & HMC-C200 (8.2 GHz) & $\begin{array}{l}\text { DRO-10.600-FR (10.6 } \\
\text { GHz) }\end{array}$ & $\begin{array}{l}\text { Ultra-low-noise } \\
\text { STALO (10 GHz) }\end{array}$ \\
\hline 100 & -62 & -59 & -106.9 \\
\hline $1 \mathrm{k}$ & -95 & -87 & -138.1 \\
\hline $10 \mathrm{k}$ & -122 & -112 & -163.8 \\
\hline $100 \mathrm{k}$ & -137 & -168.8 \\
\hline $1 \mathrm{M}$ & -140 & -160 & -173.1 \\
\hline
\end{tabular}


When comparing two oscillators' phase noise performance operating at different frequencies, a $20 \log N$ correction must be made to the phase noise power, where $N$ is the multiplication factor [6]. A correction of $1 \mathrm{~dB}$ must be made to the phase noise levels of the HMC-C200, a $-1.23 \mathrm{~dB}$ to the $D R O-10.600-F R$ and a $-0.72 \mathrm{~dB}$ to the ultra-low-noise STALO. The phase noise performances of the five oscillators, translated to $9.2 \mathrm{GHz}$, are listed in Table 4-9. The phase noise of these oscillators are also plotted together in Figure 4.36.

Table 4-9: Comparison of commercially available high performance oscillators and ultra-low-noise STALO with oscillator designed in this thesis, translated to $9.2 \mathrm{GHz}$ operating frequency.

\begin{tabular}{|l|l|l|l|l|l|}
\hline & \multicolumn{5}{|c|}{ Phase noise power [dBc/Hz] @ 9.2 GHz operating frequency } \\
\hline $\begin{array}{l}\text { Offset } \\
\text { frequency } \\
{[\mathrm{Hz}]}\end{array}$ & HMC-C200 & $\begin{array}{l}\text { DRO-10.600- } \\
\text { FR }\end{array}$ & $\begin{array}{l}\text { Ultra-low- } \\
\text { noise } \\
\text { STALO }\end{array}$ & $\begin{array}{l}\text { Oscillator using } \\
\text { HMC451LC3 } \\
\text { amplifier }\end{array}$ & $\begin{array}{l}\text { Oscillator } \\
\text { using DB01- } \\
\text { 0485 } \\
\text { amplifier }\end{array}$ \\
\hline 100 & -61 & -60.2 & -107.6 & -59.6 & -66.9 \\
\hline $1 \mathrm{k}$ & -94 & -88.2 & -138.8 & -88.2 & -91.5 \\
\hline $10 \mathrm{k}$ & -121 & -113.2 & -164.5 & -114 & -114.8 \\
\hline $100 \mathrm{k}$ & -139 & -138.2 & -169.5 & -137.8 & -146.1 \\
\hline $1 \mathrm{M}$ & -149 & -161.2 & -173.8 & -161.6 & -162.3 \\
\hline
\end{tabular}

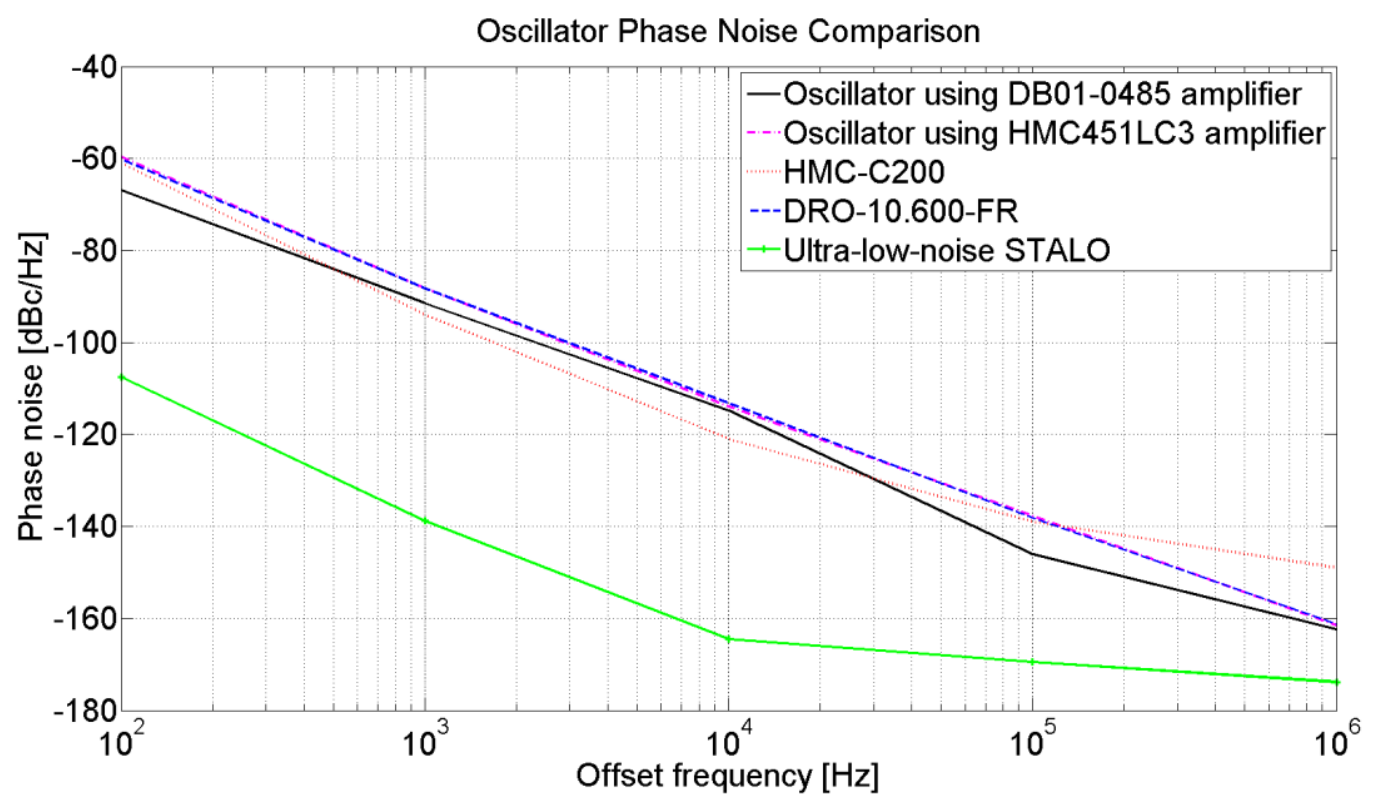

Figure 4.36: Phase noise comparison of commercially available, low noise, high performance oscillators, ultra-low-noise STALO and the oscillator designed in this thesis.

The above plot reveals that the phase noise performance of the oscillator designed in this thesis, using a medium power active device with a high noise figure and a high flicker corner frequency, is comparable to commercially available, low noise, high performance oscillators. By using an active device with a lower flicker corner frequency and lower noise figure, the phase noise performance of the oscillator is further improved. 
The ultra-low-noise STALO shows excellent phase noise performance compared to the other oscillators and is much more complex than the oscillator designed in this thesis. It should be noted that this oscillator is temperature controlled and the cavity operates in the $\mathrm{TE}_{023}$ mode which has a higher unloaded Q-factor. Operation in this high Q mode results in a bigger physical structure. 


\section{Chapter 5}

\section{Conclusions and recommendations}

\subsection{Conclusions}

The objective of this project was to design and measure a low phase noise oscillator, using a metallic waveguide cavity as the frequency selective element and performing phase noise measurements with the output power taken at different locations in the oscillator loop.

By obtaining the correct coupling values and operating the cylindrical waveguide cavity in the high- $\mathrm{Q}$ $\mathrm{TE}_{011}$ mode, the resonator was designed to ensure the best phase noise performance. To tune the operating frequency of the $T E_{011}$ mode and separate the degenerate $T E_{011}$ and $T M_{111}$ modes, a tuning screw was inserted at one of the endplates of the cavity extending in the axial direction. Inserting this screw had little effect on the loaded Q-factor of the resonator.

The spurious modes emanating from the resonator was successfully suppressed by inserting a filter into the oscillator loop.

It was found that by taking the output of the oscillator directly after the resonator can substantially improve the phase noise performance at offset frequencies greater than the Leeson frequency. Phase noise performance is also substantially improved by using a low noise voltage regulator to power the active device and by using an active device with a low noise figure and low flicker corner frequency.

By using the active device that has a low noise figure and low flicker corner frequency, a phase noise level of $-146.1 \mathrm{dBc} / \mathrm{Hz}$ at $100 \mathrm{kHz}$ offset was obtained. At $10 \mathrm{kHz}$ offset, a phase noise level of $-114.8 \mathrm{dBc} / \mathrm{Hz}$ was obtained and can further be improved by using a low noise voltage regulator to power the active device.

It was illustrated that by operating the oscillators with a small gain margin, Leeson's prediction for oscillator phase noise is valid.

A low phase noise waveguide cavity oscillator was designed and two were built for phase noise measurements. Simulations and measurements were in excellent agreement. Outstanding phase noise performance was obtained and is comparable to commercially available, low noise, high performance oscillators whose components are not cryogenically cooled.

\subsection{Recommendations for future work}

Frameworks for designing a classical low phase noise oscillator have been set out. To further improve phase noise performance, different active devices should be investigated to use in the oscillator loop. High power, low noise figure and low flicker corner frequency are important parameters of the active device to ensure improved phase noise performance. 
CONCLUSIONS AND RECOMMENDATIONS

Phase noise performance can substantially be improved by making use of a STALO configuration. These configurations are somewhat more complex, but the end result shows superb phase noise performance. 


\section{Bibliography}

[1] David M. Pozar, Microwave and RF design of wireless systems.: John Wiley \& Sons, Inc., 2000 .

[2] James A. Barnes et al., "Characterization of Frequency Stability," Instrumentation and Measurement, IEEE transactions on, vol. IM-20, no. 2, pp. 105 - 120, May 1971.

[3] Randall W. Rhea, Oscillator Design and Computer Simulation, 2nd ed.: Noble Publishing Corporation, 2000.

[4] Z. Galani et al., "Analysis and design of a single-resonator GaAs FET oscillator with noise degeneration," Microwave theory and techniques, IEEE transactions on, vol. 32, no. 12, pp. 1556-1565, 1984.

[5] George D. Vendelin, Anthony M. Pavio, and Ulrich L. Rohde, Microwave Circuit Design Using Linear and Non Linear Techniques.: John Wiley \& Sons, Inc, 1990.

[6] David M. Pozar, Microwave Engineering, 3rd ed.: John Wiley \& Sons, Inc., 2005.

[7] Paul H. Young, Electronic Communication Techniques, 3rd ed.: Merrill, 1994.

[8] Enrico Rubiola, Phase noise and frequency stability in oscillators.: Cambridge University Press, 2009.

[9] microwaves101. [Online]. www.microwaves101.com

[10] Darko Kajfez, "Q factor measurements, analog and digital," 1999.

[11] John van der Merwe, "An experimental investigation into the validity of Leeson's equation for low phase noise oscillator design," 2010.

[12] B.P Lathi, Modern digital and analog communication systems, 3rd ed. New York: Oxford University Press, 1998.

[13] "IEEE Standard Definitions of Physical Quantities for Fundamental Frequency and Time Metrology-Random Instabilitites," IEEE Std 1139-1999, 1999.

[14] Henderikus Jan Moes, "A Low noise PLL-base frequency synthesiser for X-band radar," University of Stellenbosch, Stellenbosch, 2008.

[15] D.B. Leeson, "A simple model of feedback oscillator noise spectrum," Proceedings of the IEEE, vol. 54, no. 2, pp. 329-330, February 1966. 
[16] Elrien Vermaak, "Development of a low phase noise microwave voltage controlled oscillator," 2008.

[17] J. Rutman, "Relations between spectral purity and frequency stability," in 28th Annual Symposium on Frequency Control. 1974, 1974, pp. 160-165.

[18] G.J. Dick and J. Saunders, "Microwave oscillator with reduced phase noise by negative feedback incorporating microwave signals with suppressed carrier," In its The Telecommunications and Data Acquisition Report, pp. 20-33, 1989.

[19] G,J. Dick and J. Saunders, "Measurement and analysis of a microwave oscillator stabilized by a sapphire dielectric ring resonator for ultra-low noise," Ultrasonics, Ferroelectrics and Frequency, IEEE transactions on, vol. 37, no. 5, pp. 339-346, September 1990.

[20] J. Saunders and T. Tucker, "Ultra-low noise microwave phase stabilizer using sapphire ring resonator," in Frequency control, 1990, Proceedings of the 44th Annual symposium on, 1990, pp. 577-584.

[21] A. Sen Gupta et al., "High-spectral-purity microwave oscillator: Design using conventional air-dielectric cavity," in Proceedings of the 2003 IEEE International Frequency Control Symposium and PDA exhibition jointly with the 17th European Frequency and Time forum, 2003, pp. 423-430.

[22] C.W. Nelson, D.A. Howe, and A. Sen Gupta, "Ultra-low-noise cavity-stabilized microwave reference oscillator using an air-dielectric resonator," in 36th Annual Precise Time and Time Interval (PTTI) Meeting, 2007, pp. 173-178.

[23] Constantine A. Balanis, Advanced Engineering Electromagnetics.: John Wiley \& Sons, 1989.

[24] C.S. Lee, S.W. Lee, and S.L. Chaung, "Plot of modal field distribution in rectangular and circular waveguides," Microwave theory and techniques, IEEE Transactions on, vol. 33, no. 3, pp. 271-274, March 1985.

[25] Robert E. Collin, Foundations for Microwave engineering, 2nd ed. New York: Wiley-IEEE press, 2001.

[26] Simon Ramo, John R. Whinnery, and Theodore Van Duzer, Fields and waves in communication electronics, 3rd ed.: John Wiley \& Sons, Inc., 1993.

[27] P. Meyer, Microwave networks 813 slides.

[28] H.A. Bethe, "Theory of diffraction by small holes," vol. 66, pp. 163-182, October 1944.

[29] N.A. McDonald, "Electric and magnetic coupling through small apertures in shield walls of any thickness," Microwave theory and techniques, IEEE transactions on, vol. 20, no. 10, 
pp. 689-695, October 1972.

[30] George L. Matthaei, Leo Young, and E.M.T. Jones, Microwave filters, impedance-matching networks, and coupling structures.: Artech house, 1980.

[31] Robert E. Collin, Field theory of guided waves, 2nd ed. New York: Wiley-IEEE press, 1990. 
APPENDICES 


\title{
Appendix A
}

\section{CAD drawings}

\author{
Resonator
}




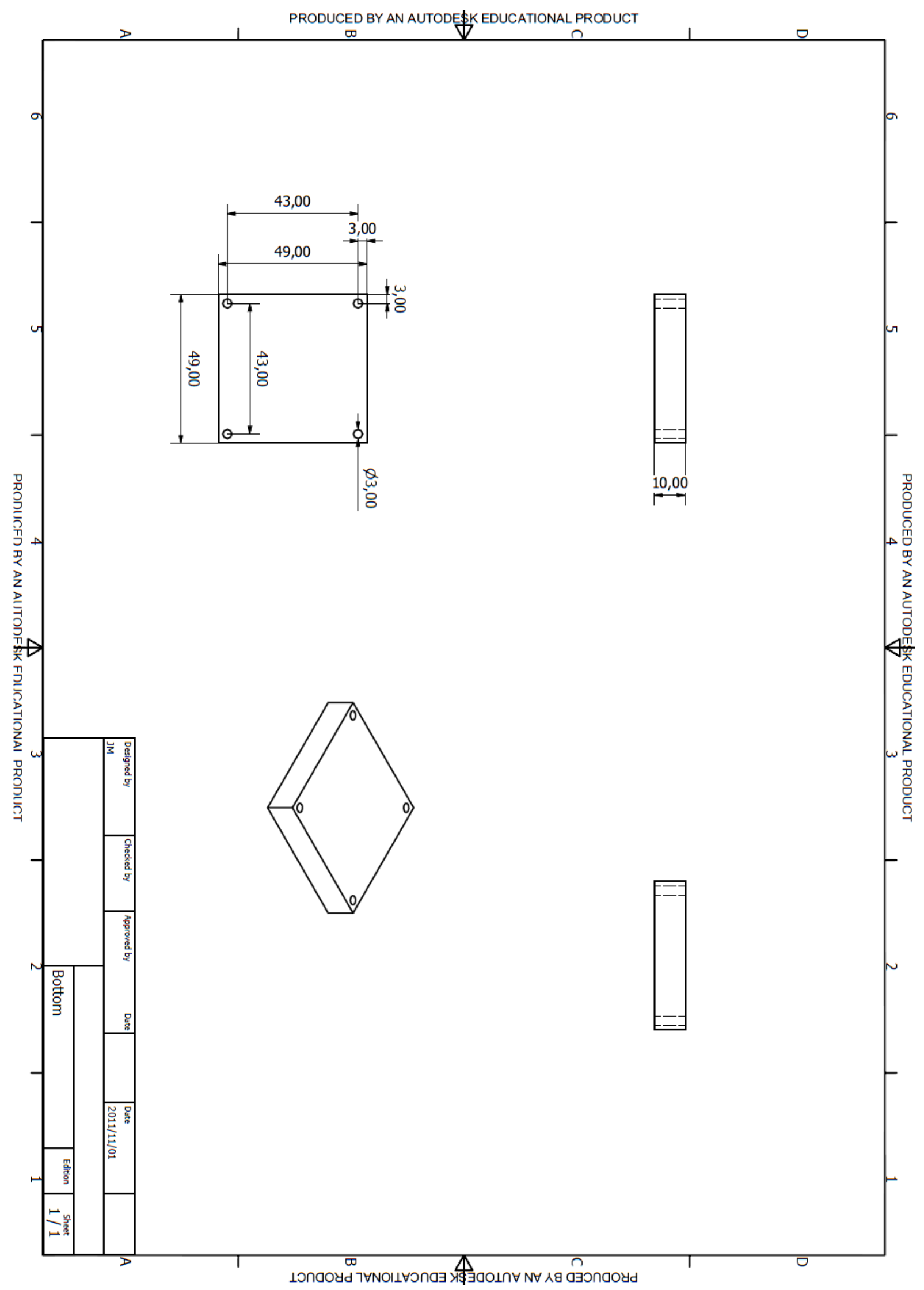




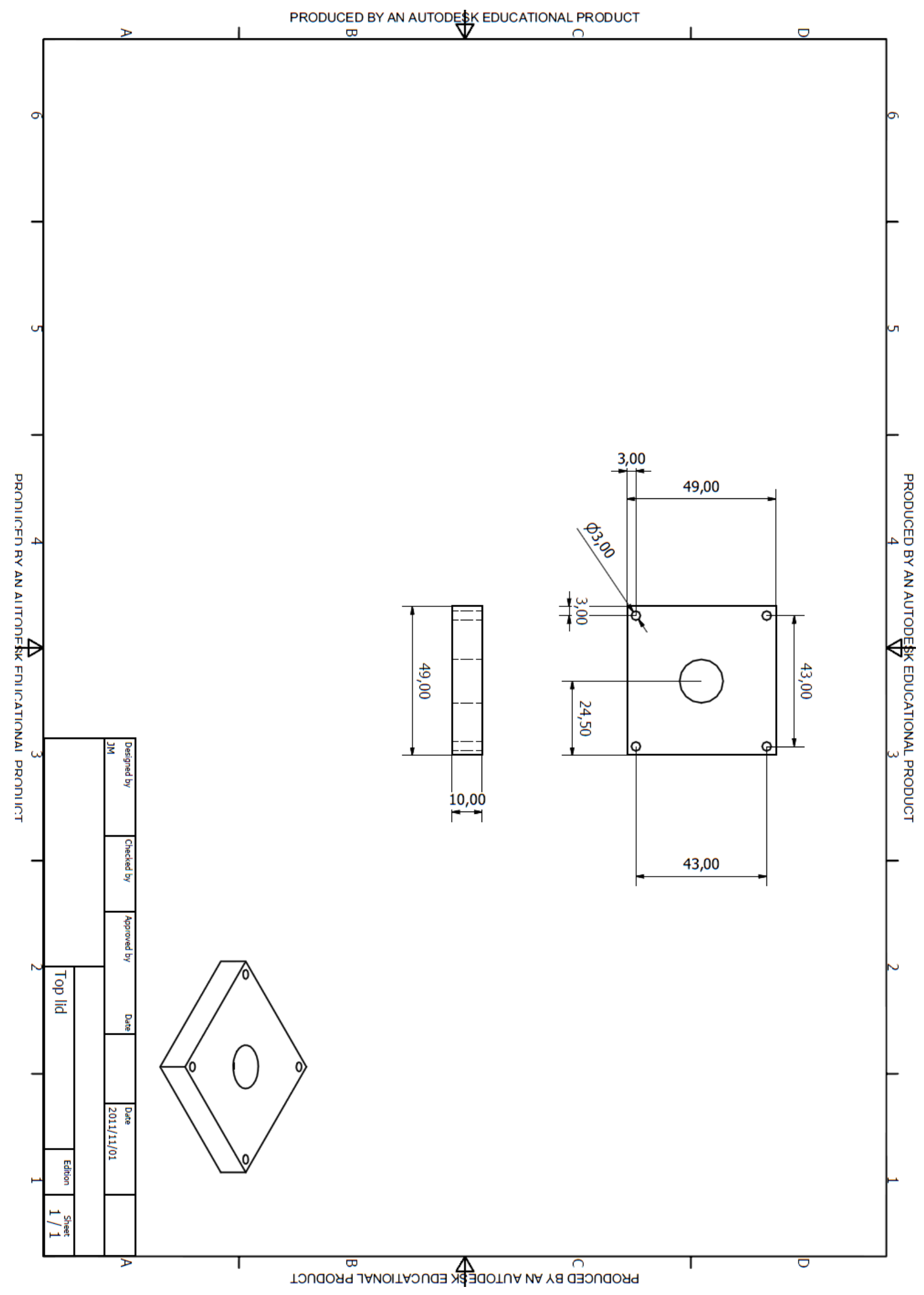




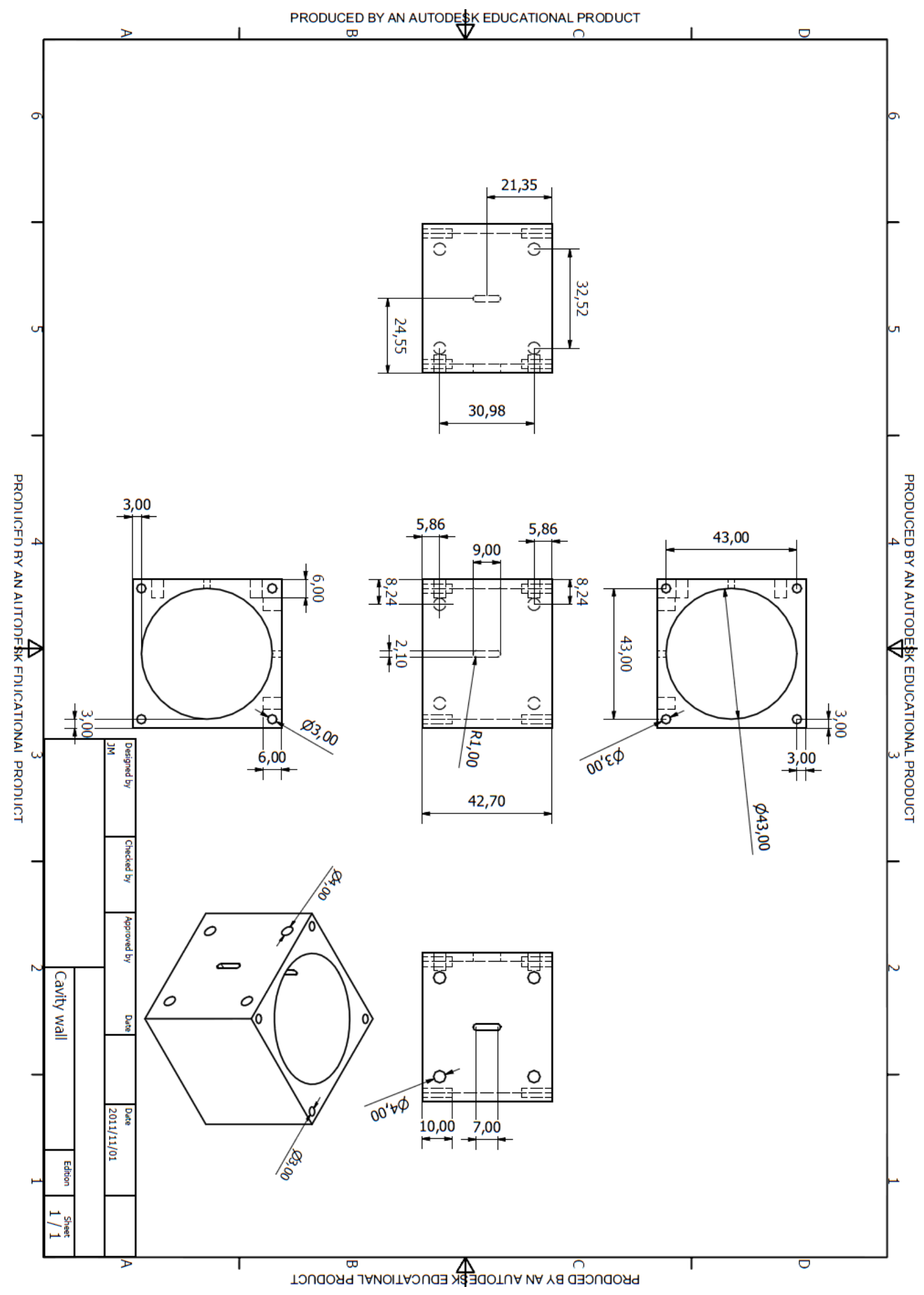




\section{Appendix B}

\section{CAD drawings}

Filter 


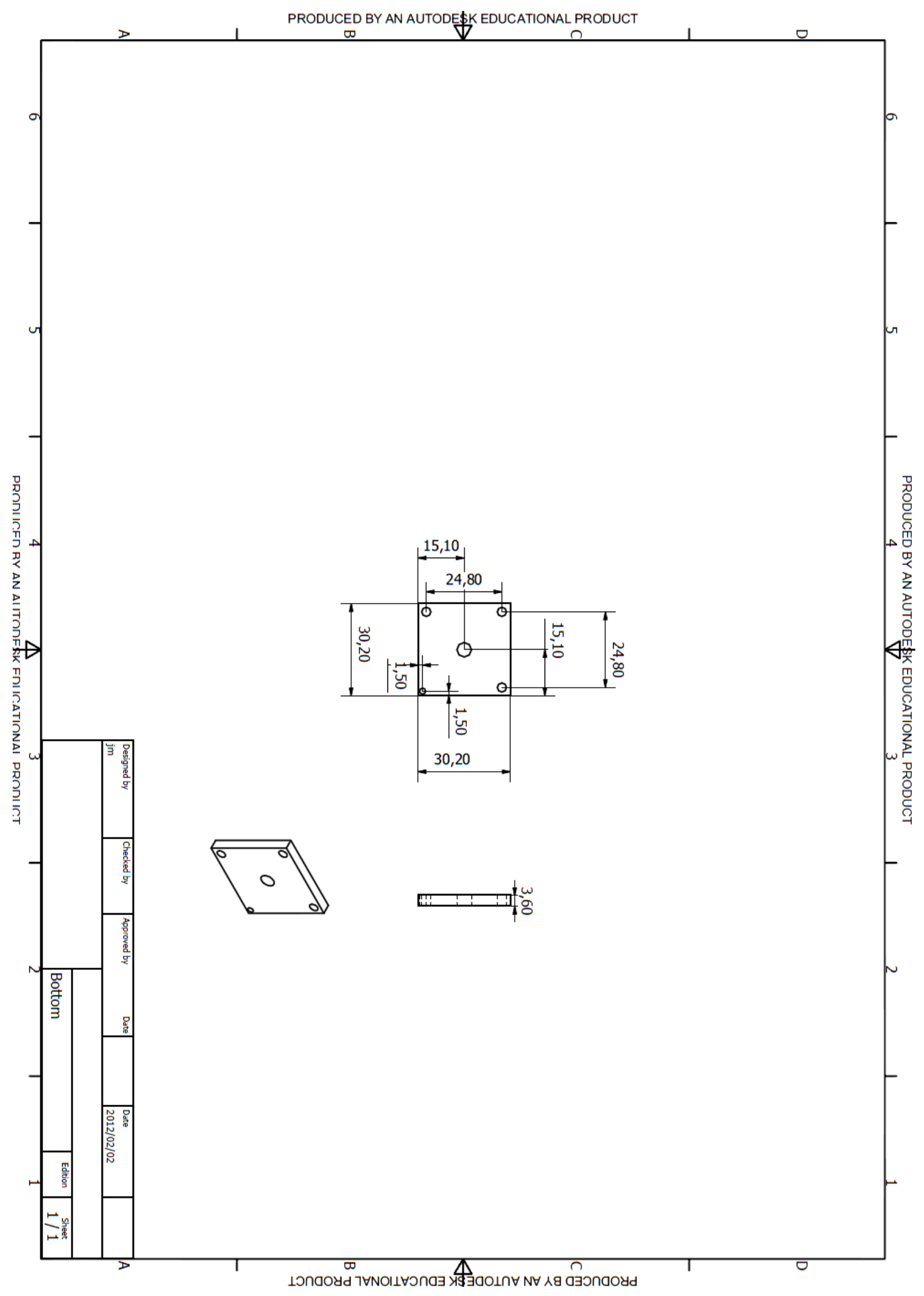




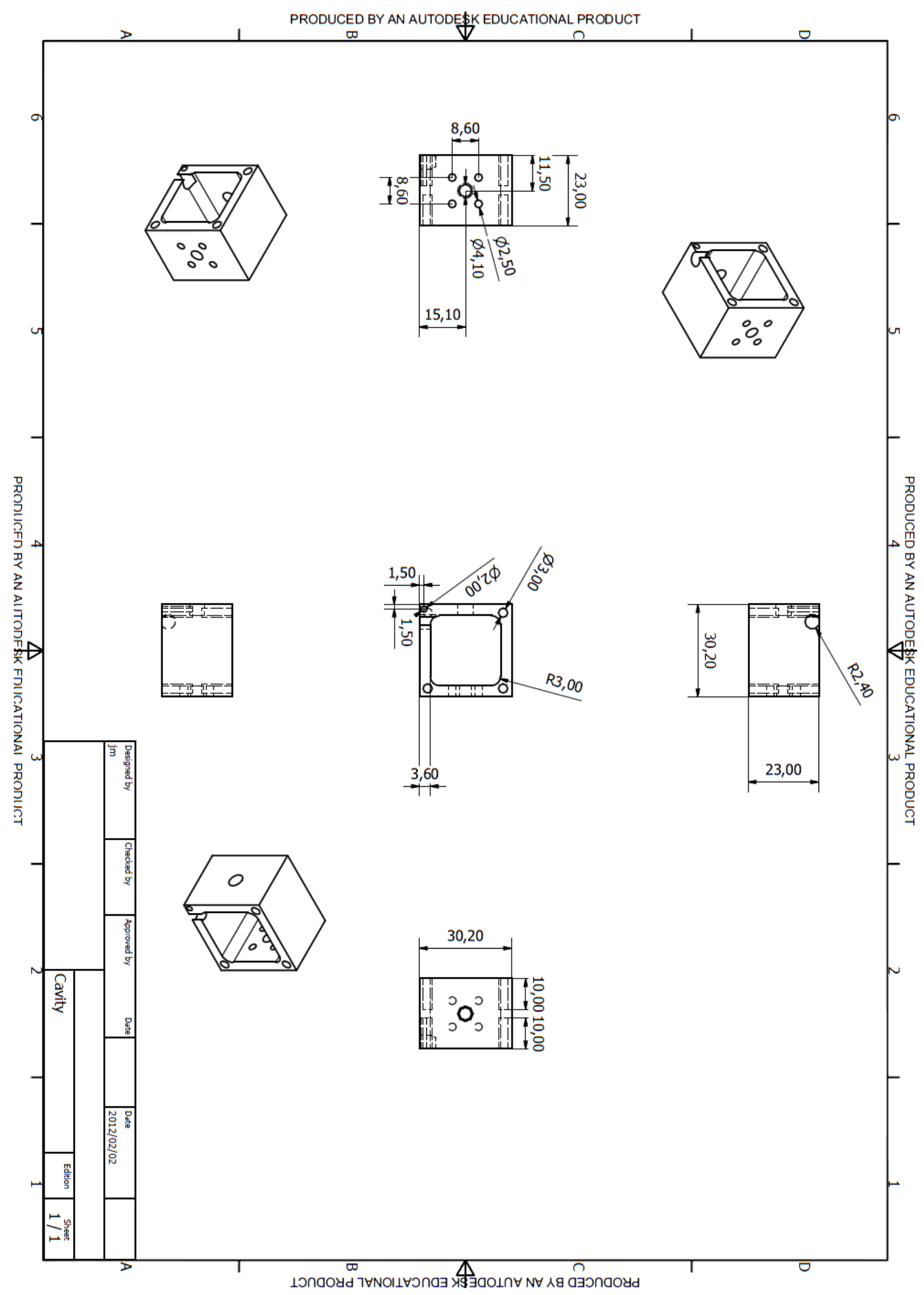




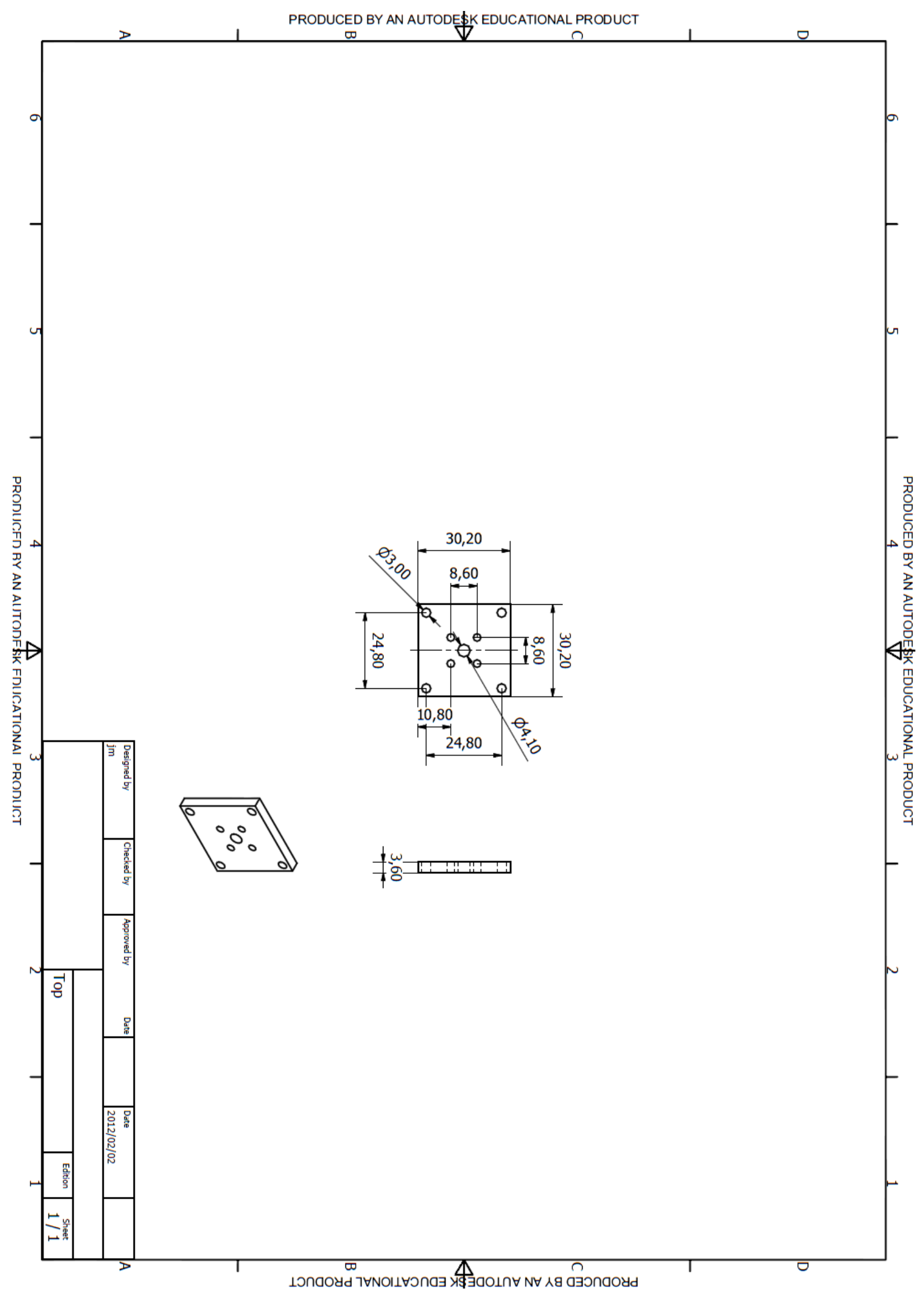




\title{
Appendix C
}

\author{
Datasheet
}

Narda DB01-0485 low noise amplifier 
DES PIN: DB01-0486

Frequency Range [ $\mathrm{GHz}$ )

Flatness ( $\pm \mathrm{dB}$ )

Small Signal Gain (dB)

Noise Figure (dB)

P1dB (dBm)

IP3 (dBm)

Input Power Handling (dBm)

VSWR (:1)

Voltage (Vdc)

Current (mA)

Spec. Temp. Range (degree C)

Case with SMAF ito

$5.0 \cdot 14.0$

1.75 max.

15 min. / 18 max.

2.7 max.

$15 \mathrm{~min}$.

25 typ.

20 max., CW

2.2/2.21\%

$+12$

150 max

$+25$

DBX -21 


\title{
Appendix D
}

\author{
Datasheet
}

Hittite HMC-C200 DRO 
DIELECTRIC RESONATOR OSCILLATOR MODULE

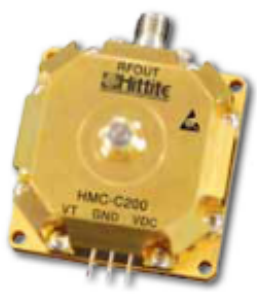

Typical Applications

The HMC-C200 DRO Module is ideal for:

- Test \& Measurement Equipment

- Lab Instrumentation

- Industrial / Medical Equipment

- Military, EW, ECM \& Communications

\section{Functional Diagram}

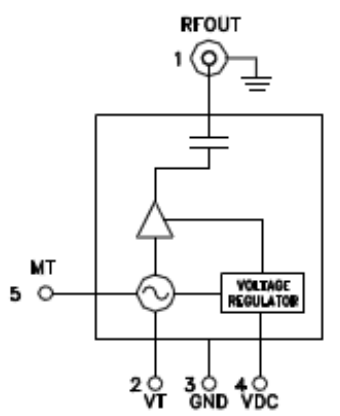

\section{Features}

Tuning Frequency: $8.0-8.3 \mathrm{GHz}$

Low SSB Phase Noise: $-122 \mathrm{dBc} / \mathrm{Hz} @ 10 \mathrm{kHz}$ Offset

Single Positive Supply: +6 to $+15 V$ @ $116 \mathrm{~mA}$

Internal Voltage Regulator

Internal Buffer Amplifier

High Output Power. +14.5 dBm

$-40{ }^{\circ} \mathrm{C}$ to $+85^{\circ} \mathrm{C}$ Operating Temperature

\section{General Description}

The HMC-C200 is a high performance dielectric resonator oscillator (DRO) that incorporates Hittite's ultra-low phase noise technology and provides $-122 \mathrm{dBc} / \mathrm{Hz}$ SSB phase noise at $10 \mathrm{kHz}$ offset. The output buffer also provides $14.5 \mathrm{dBm}$ of output power. Internal temperature compensation allows this DRO to operate over a temperature range of $-40^{\circ} \mathrm{C}$ to $+85^{\circ} \mathrm{C}$ with a frequency drift of only $2 \mathrm{ppm} /{ }^{\circ} \mathrm{C}$. The Vtune port accepts an analog tuning voltage from +2 to $+12 \mathrm{~V}$ and provides a range of $\pm 1 \mathrm{MHz}$ from the center frequency. The DRO is packaged in a small, moisture sealed 1.5" x 1.5" (36 x $36 \mathrm{~mm}$ ) module with a field replaceable SMA Connector. The HMC-C200 can be used as a drop-in module if the SMA connector is not used.

Electrical Specifications, $T_{A}=+25^{\circ} \mathrm{C}$, Frequency $=8.2 \mathrm{GHz}, \mathrm{VDC}=+6.5 \mathrm{~V}$

\begin{tabular}{|l|c|c|c|c|}
\hline \multicolumn{1}{|c|}{ Parameter } & Min. & Typ. & Max. & Units \\
\hline Frequency Range & 8.0 & 8.2 & $\mathbf{8 . 3}$ & $\mathrm{GHz}$ \\
\hline Frequency Accuracy & & \pm 250 & & $\mathrm{kHz}$ \\
\hline Power Output, Center Frequency & 12 & 13.5 & & $\mathrm{dBm}$ \\
\hline SSB Phase Noise @ 1 kHz Oftset, Center Frequency & & -95 & & $\mathrm{dBc} / \mathrm{Hz}$ \\
\hline SSB Phase Noise @ 10 kHz Offset, Center Frequency & -117 & -122 & & $\mathrm{dBc} / \mathrm{Hz}$ \\
\hline SSB Phase Noise @ 100 kHz Offset, Center Frequency & & -140 & & $\mathrm{dBc} / \mathrm{Hz}$ \\
\hline SSB Phase Noise @ 1 MHz Offset, Center Frequency & & -150 & & $\mathrm{dBc} / \mathrm{Hz}$ \\
\hline Tune Voltage (VT) & 2 & & $\mathbf{1 2}$ & $\mathbf{V}$ \\
\hline Electric Tuning Range & & \pm 1 & & $\mathrm{MHz}$ \\
\hline 2nd Harmonic (2Fo) & & -28 & & $\mathrm{dBc}$ \\
\hline 3rd Harmonic (3Fo) & & -35 & & $\mathrm{dBc}$ \\
\hline
\end{tabular}

For price, delivery and to place orders: Hittite Microwave Corporation, 2 Elizabeth Drive, Chelmsford, MA 01824 978-250-3343 tel • 978-250-3373 fax • Order On-line at www.hittite.com Application Support: Phone: 978-250-3343 or apps@hittite.com 
DIELECTRIC RESONATOR OSCILLATOR MODULE $8.0-8.3 \mathrm{GHz}$

\section{Electrical Specifications (Continued)}

\begin{tabular}{|l|c|c|c|c|}
\hline \multicolumn{1}{|c|}{ Parameter } & Min. & Typ. & Max. & Units \\
\hline Frequency Pushing & & 5 & & $\mathrm{kHz} / \mathrm{V}$ \\
\hline Frequency Pulling (into 2.0:1 VSWR) & & 5 & & $\mathrm{kHz} \mathrm{pp}$ \\
\hline Output Returm Loss & & 12 & & $\mathrm{~dB}$ \\
\hline Frequency Drift Rate (Temperature) & & 2 & & $\mathrm{ppm} /{ }^{\circ} \mathrm{C}$ \\
\hline Voltage Supply (VDC) & 6 & & 15 & $\mathrm{~V}$ \\
\hline Supply Current \& VDC $=+6.5 \mathrm{~V}$ & & 116 & & $\mathrm{~mA}$ \\
\hline Frequency Drift vs. Time $625^{\circ} \mathrm{C}$ & & 1 & & $\mathrm{ppm} /$ year \\
\hline
\end{tabular}

Phase Noise vs. Temperature@ $8.2 \mathrm{GHz}$

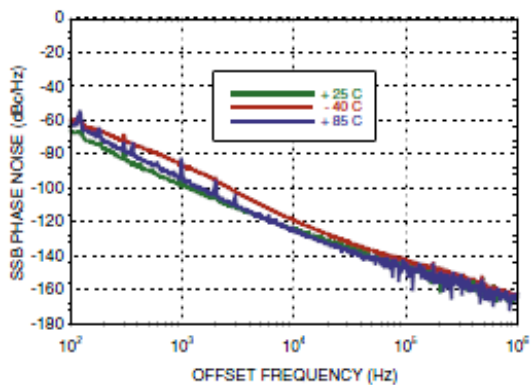

Output Power vs. Temperature @ $8.2 \mathrm{GHz}$

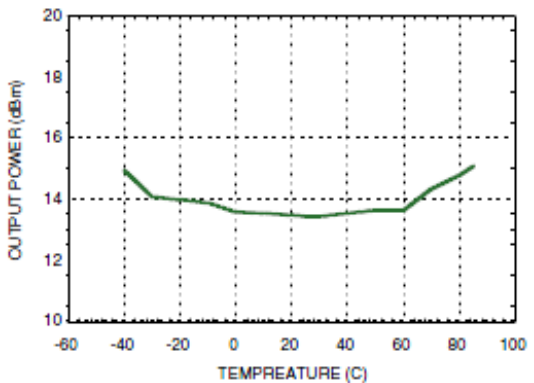

\section{Output Frequency vs. Temperature}

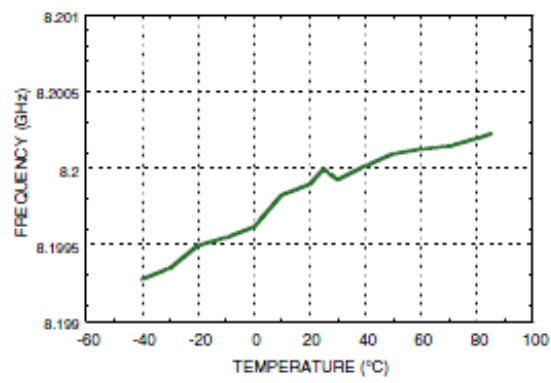

VDC Current vs. Temperature @ $8.2 \mathrm{GHz}$

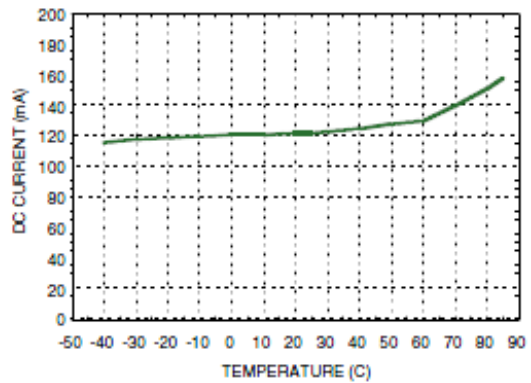

For price, delivery and to place orders: Hittite Microwave Corporation, 2 Elizabeth Drive, Chelmsford, MA 01824 978-250-3343 tel • 978-250-3373 fax • Order On-line at www.hittite.com 
Frequency \& Power vs. Tuning Voltage ${ }^{[1]}$

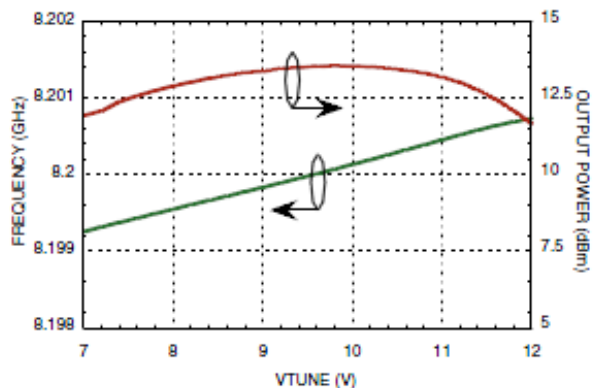

[1] For DRO tuned to $9.5 \mathrm{~V}$ nominal VT

\section{Absolute Maximum Ratings}

\begin{tabular}{|l|l|}
\hline VDC & $+15 \mathrm{~V}$ \\
\hline VT & $010+15 \mathrm{~V}$ \\
\hline Storage Temperature & -65 to $+150^{\circ} \mathrm{C}$ \\
\hline Operating Temperature & -40 to $+85^{\circ} \mathrm{C}\left[{ }^{2]}\right.$ \\
\hline
\end{tabular}

\section{Vibration Sensitivity}

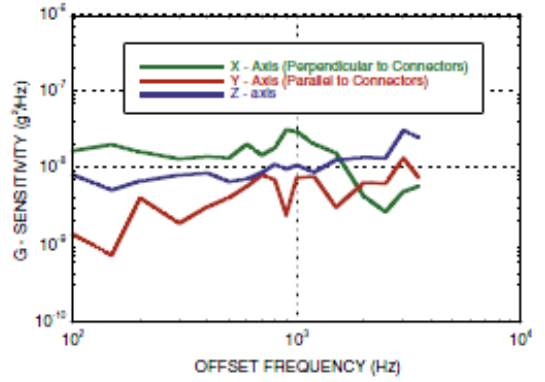

[2] While the HMC-C200 will operate over this temperature range, it is not guaranteed that the specific frequency will $b_{\theta}$ in the tuning range at all temperatures. Contact the factory for information on additional screening to ensure any required operation over temperature.

\section{Pin Descriptions}

\begin{tabular}{|c|c|c|c|}
\hline Pin Number & Function & Description & Interface Schematic \\
\hline 1 & RFOUT & RF output (AC coupled) uses a female SMA connector. & RFour o-f1 \\
\hline 2 & VT & $\begin{array}{l}\text { Control Voltage and Modulation Input. Modulation band- } \\
\quad \text { width } 100 \mathrm{kHz} \text { for ideal source. } .^{33}(+2 \mathrm{~V} \text { to }+12 \mathrm{~V})\end{array}$ & 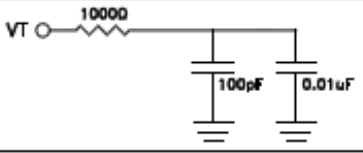 \\
\hline 3 & GND & Must be connected to power supply ground. & $\stackrel{9 \mathrm{GND}}{I}^{=}$ \\
\hline 4 & VDC & Supply Voltage $\mathrm{Vdc}=+6 \mathrm{~V}$ to $+15 \mathrm{~V}$. & 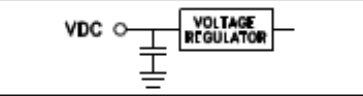 \\
\hline 5 & MT & $\begin{array}{l}\text { Mechanical Tuning Screw. } \\
\text { (This is initially set at the factory per customer specified } \\
\text { frequency, See Application Notes herein) }\end{array}$ & мro $\prod^{\prime}$ \\
\hline
\end{tabular}

[3] For higher modulation bandwidths, contact the factory

\section{Ordering Instructions}

Each $\mathrm{HMC}-\mathrm{C} 200 \mathrm{DRO}$ requires tuning to a specific frequency in the $8.0-8.3 \mathrm{GHz}$ range. To order an $\mathrm{HMC}-\mathrm{C} 200$, this frequency must be specified at the time of purchase. Hittite guarantees a $\pm 250 \mathrm{kHz}$ range around this specified frequency upon delivery. Please refer to the Application Notes for more details. For example, when ordering an HMC-C200 centered at $8.200 \mathrm{GHz}$, specify HMC-C200-8200.

For price, delivery and to place orders: Hittite Microwave Corporation, 2 Elizabeth Drive, Chelmsford, MA 01824 978-250-3343 tel • 978-250-3373 fax • Order On-line at www.hittite.com Application Support: Phone: $978-250-3343$ or apps@hittite.com 
Outline Drawing

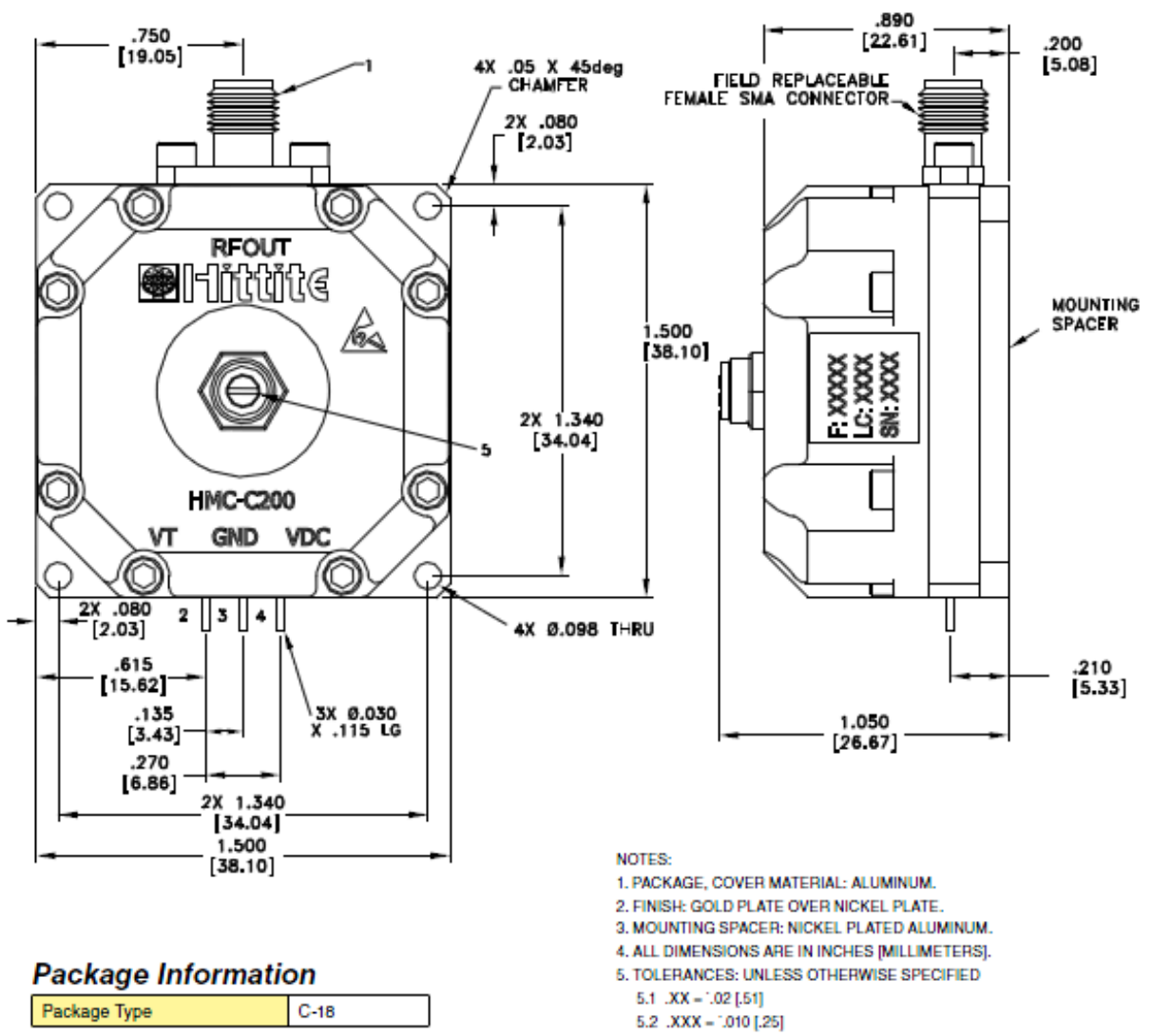




\title{
DIELECTRIC RESONATOR OSCILLATOR MODULE
}

\author{
$8.0-8.3 \mathrm{GHz}$
}

\section{Application Notes \\ DRO Tuning Procedure:}

1. A mechanical tuning screw (MT) is provided that can adjust the center frequency by approximately $\pm 20 \mathrm{MHz}$ from the factory setting. This screw is set at factory per customer specified frequency. Adjusting the screw clockwise increases the frequency while turning the screw counterclockwise decreases the frequency. The mechanical tuning screw (MT) has stops at either end of its approximately 8 turn range. Do not attempt to force screw the past stops.

2. Connect the RF output to a Spectrum Analyzer and adjust the mechanical tuner (MT) to set the output frequency to within $1 \mathrm{MHz}$ of the desired value.

3. Set Spectrum Analyzer settings for a $10 \mathrm{MHz}$ span centered on the desired output frequency. Change the Reference Value of the Spectrum Analyzer to be approximately $15 \mathrm{dBm}$ and change the vertical scale to be $1 \mathrm{~dB} /$ division such that the peak oscillation frequency is visible on the screen.

4. Use the tune voltage (VT) to maximize the DRO output power and note the frequency. Note: The tune voltage should never be adjusted higher than $+15 \mathrm{~V}$.

5. If the frequency is meeting the new requirement and the output power is greater than $12 \mathrm{dBm}$, then the tuning procedure is complete. If the frequency is not meeting the requirement then carefully readjust the mechanical tuner (MT) and tune voltage (VT) until the output frequency is within the required range.

\section{Please Note:}

1. The tune voltage (VT) sustains oscillation over a fairly narrow range (approximately $1 \mathrm{MHz}$ ) before output power, temperature stability and phase noise performance starts to degrade. In some cases it is possible for the tune voltage (VT) to be adjusted to the point where oscillation stops. Therefore, the mechanical tuning screw (MT) should be used as a coarse tune and the tune voltage (VT) is effectively a fine tune. In PLL applications where the tune voltage (VT) is used within the loop, this limited voltage range must be considered during the design of the loop. A limiting circuit must be used to keep the VT voltage within the optimal range.

2. If the output power is less than $12 \mathrm{dBm}$ then the DRO may be at the correct frequency but the phase noise performance and the stability over temperature may both have been degraded. It is therefore recommended that the customer verify phase noise and stability after custom tuning.

For price, delivery and to place orders: Hittite Microwave Corporation, 2 Elizabeth Drive, Chelmsford, MA 01824 978-250-3343 tel • 978-250-3373 fax • Order On-line at www.hittite.com Application Support: Phone: $978-250-3343$ or apps@hittite.com 


\section{Fixed Output Frequency Application}

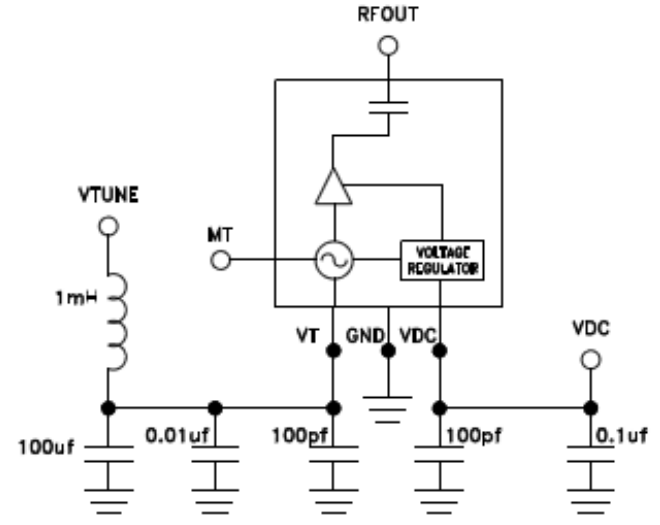

\section{Phase Locked Application}

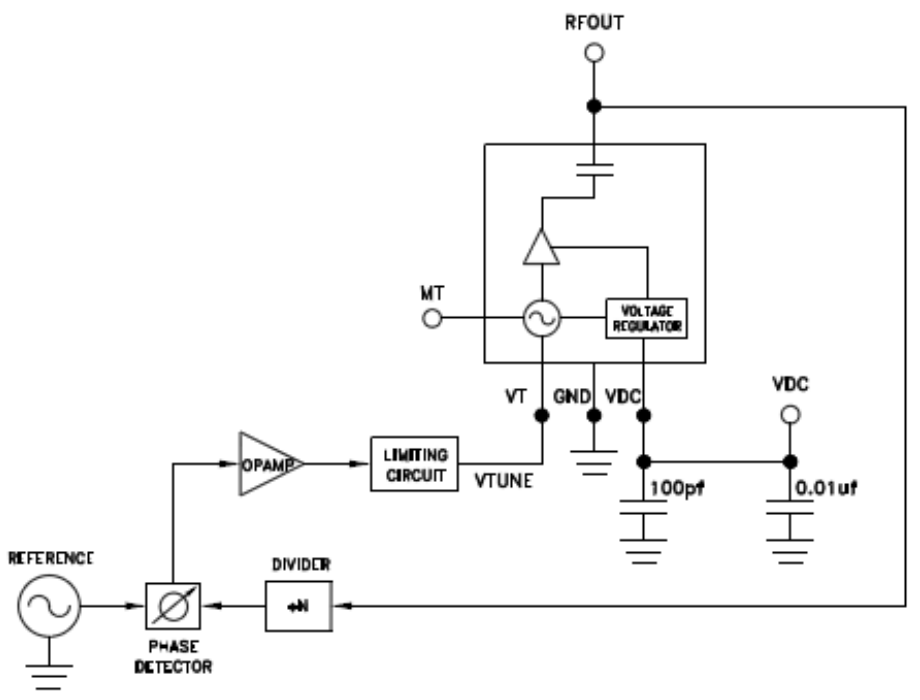

For price, delivery and to place orders: Hittite Microwave Corporation, 2 Elizabeth Drive, Chelmsford, MA 01824 978-250-3343 tel - 978-250-3373 fax - Order On-line at www.hittite.com 


\title{
Appendix E
}

\author{
Datasheet
}

\section{PSI DRO-10.600-FR}




\section{SPECIFICATIONS}

1. Output Frequency:

$10.600 \mathrm{GHz}$

2. Factory Set Accuracy:

$<+/-4$ ppm typical (free running).

3. Output Power:

$=+12 \mathrm{dBm}$

4. Discrete Spurious Tones:

fm $=10 \mathrm{kHz}$ : Less than $+30-30 \log (\mathrm{fm}) \mathrm{dBc}$

$10 \mathrm{kHz}<$ fm $<1 \mathrm{MHz}:-90 \mathrm{dBc}$

5. Phase Noise (Free Running):

\begin{tabular}{|c|c|c|}
\hline Offset & Guaranteed & Typical \\
\hline (a) $100 \mathrm{~Hz}$ & $-54 \mathrm{dBc} / \mathrm{Hz}$ & $-59 \mathrm{dBc} / \mathrm{Hz}$ \\
\hline (a) $1 \mathrm{kHz}$ & $-84 \mathrm{dBc} / \mathrm{Hz}$ & $-87 \mathrm{dBc} / \mathrm{Hz}$ \\
\hline (a) 10 kHz & $-109 \mathrm{dBc} / \mathrm{Hz}$ & $-112 \mathrm{dBc} / \mathrm{Hz}$ \\
\hline (a) $100 \mathrm{kHz}$ & $-132 \mathrm{dBc} / \mathrm{Hz}$ & $-137 \mathrm{dBc} / \mathrm{Hz}$ \\
\hline (a) $1 \mathrm{MHz}$ & $-151 \mathrm{dBc} / \mathrm{Hz}$ & $-160 \mathrm{dBc} / \mathrm{Hz}$ \\
\hline (a) $6 \mathrm{MHz}$ & $-154 \mathrm{dBc} / \mathrm{Hz}$ & $-163 \mathrm{dBc} / \mathrm{Hz}$ \\
\hline
\end{tabular}

Specified Phase Noise is quoted as a "single oscillator" measurement and equals the measured two oscillator noise with $3 d B$ subtracted, unless noted otherwise

6. Amplitude Noise: Amplitude Noise is not currently guaranteed. Typical AM noise is:

$\begin{array}{ll}\text { Offset } & \text { Typical } \\ (100 \mathrm{~Hz} & -140 \mathrm{dBc} / \mathrm{Hz} \\ @ 1 \mathrm{kHz} & -150 \mathrm{dBc} / \mathrm{Hz} \\ @ 10 \mathrm{kHz} & -155 \mathrm{dBc} / \mathrm{Hz} \\ @ 100 \mathrm{kHz} & -160 \mathrm{dBc} / \mathrm{Hz} \\ @ 1 \mathrm{MHz} & -165 \mathrm{dBc} / \mathrm{Hz} \\ @ 6 \mathrm{MHz} & -165 \mathrm{dBc} / \mathrm{Hz}\end{array}$

7. Long Term Stabilitv: Not specified, but approximately $2 \mathrm{ppm} /{ }^{\circ} \mathrm{C}$ after 15 minutes warm up.

8. Phase Locking: The -FR oscillator is a Free Running Oscillator. It has a VCO input with a range from approximately -3.5 Volts to +8 Volts.

(The VCO feature is included to allow Phase Locking of the DRO for verification of the oscillator's phase noise.)
9. VCO Input:
$-3.5 \mathrm{~V}$ to $+8 \mathrm{~V}$
10. Electronic Tuning Range:
$> \pm 100 \mathrm{kHz}$
11. Storage Environment:
40 to $+71^{\circ} \mathrm{C}$
Relative humidity 0 to $100 \%$ condensing.
12. Operating Environment:
$0^{\circ}$ to $+45^{\circ} \mathrm{C}$ (Non Condensing)
13. Weight:
Is frequency dependent, but is approx. $300 \mathrm{gm}$ 


\section{Electronic Outouts:}

\section{PSI}

Microwave Outputs: 50 Ohms, VSWR 1.30 max., SMA(F) Connector VCO Input: $\quad 50 \mathrm{Ohms}$, VSWR $1.30 \mathrm{max}$., SMA(F) Connector Power: $\quad$ Solder type DC Feed-through

15. User Sunnlied Power Sunnly: $\quad+12$ to $+15 \mathrm{VDC}$, Gnd.

Ripple must be less than $5 \mathrm{mV} \mathrm{p}-\mathrm{p}, 10 \mathrm{~Hz}$ to $20 \mathrm{MHz}$

NOTE: To ensure phase noise specification is met, and a low spurious content, the power supply must be a linear supply, with low voltage noise.

16. Dimensions:
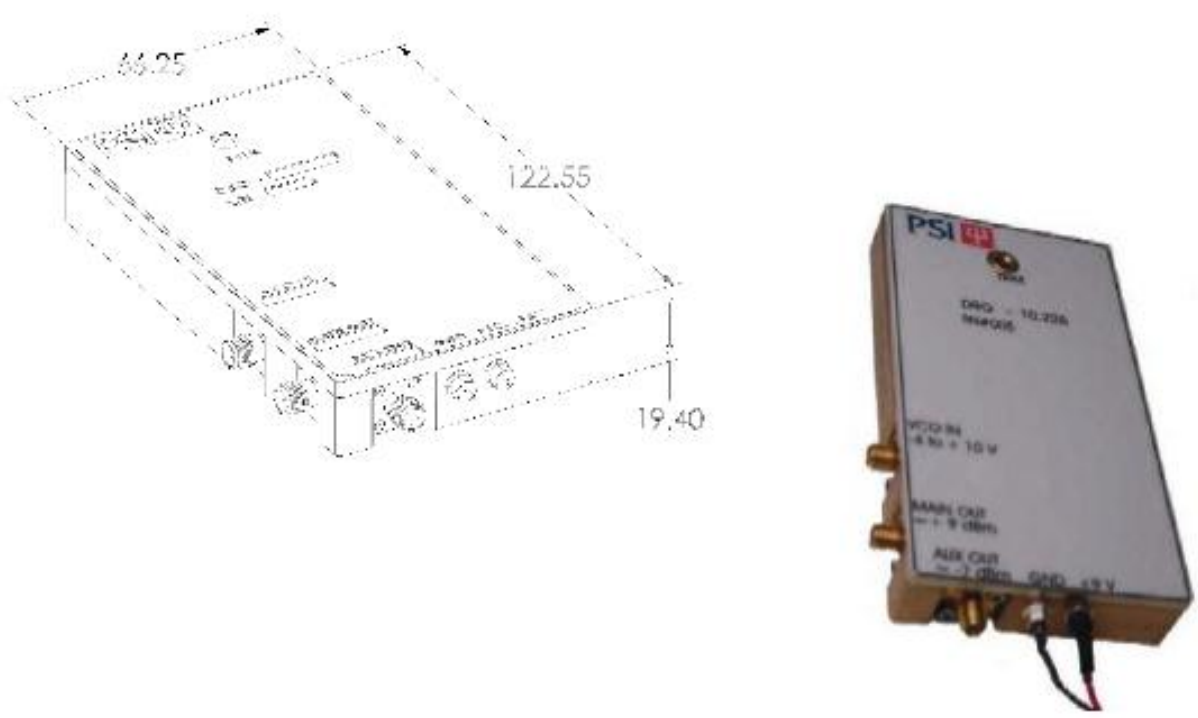


\section{Low Phase Noise DROs}

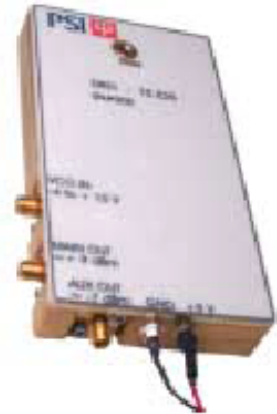

Poseidon's expertise in ultra-low phase noise sapphire-loaded cavity oscillators is now brought to bear in high performance, low phase noise DROs.

The excellent phase noise performance offered by the PSI range of DROs make them suitable for applications including:

- low-jitter communication systems

- low cost reference oscillators for phase noise measurements

- radar systems

- digital radio (QAM)

- LMDS

Available in frequencies from 8 to $12 \mathrm{GHz}$, the PSI range of DROs are available in three configurations:

- free running, with mechanical and electrical frequency tuning, or

- with integral phase lock loop circuit for a user-supplied reference signal, or - with integral phase lock loop circuit and low noise crystal reference, providing a compact, low phase noise reference oscillator.

All PSI DROs feature internal voltage regulation for immunity to power supply noise. These high performance DROs are available at an affordable price.

\section{PSI}

Unit 1, 95 Queen Victoria Street Fremantle WA 6160 Australia

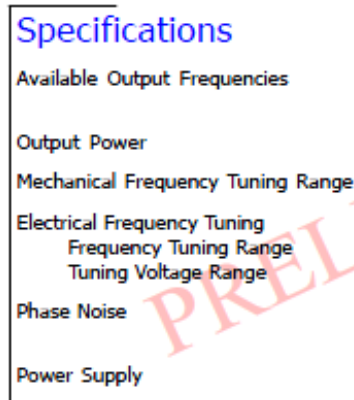

A PLL circuit is provided to phase lock the DRO to a user-supplied reference signal. The reference signal may be specified as $10 \mathrm{MHz}$ or $100 \mathrm{MHz}$. Consult PSI for other reference frequencies.

\section{Phase Lock with Crystal Reference (-XPL)}

A PLL circuit and a $100 \mathrm{MHz}$ low-noise crystal reference is integrated into the DRO. The DRO is phase locked to the crystal, improving phase noise performance at offsets less than $100 \mathrm{kHz}$ from the carrier. The $100 \mathrm{MHz}$ low noise crystal signal is available as an output.

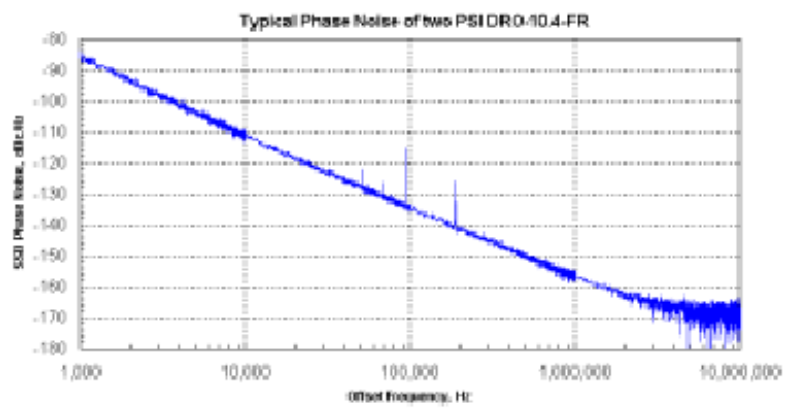

Poseidon Scientific Instruments

Phone: +61 894306639 Fax: +61893354650
Email: sales@psi.com.au Web: www.psi.com.au 